Studien aus dem Max-Planck-Institut

für Sozialrecht und Sozialpolitik

Ulrich Becker/Elisabeth Wacker/Minou Banafsche (Hrsg.)

Inklusion und Sozialraum

Behindertenrecht und Behindertenpolitik in der Kommune 


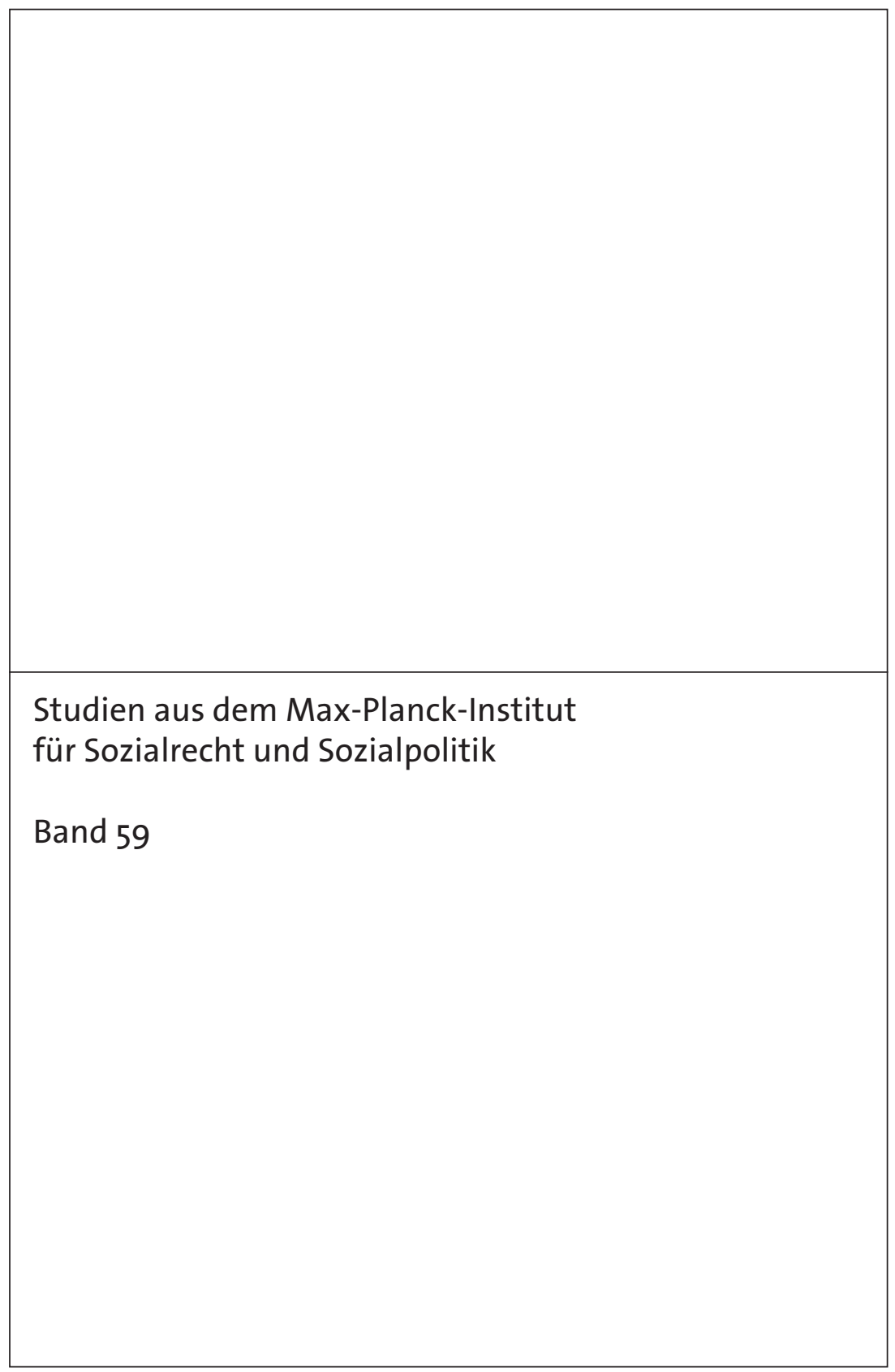


Prof. Dr. Ulrich Becker/Prof. Dr. Elisabeth Wacker/

Dr. Minou Banafsche (Hrsg.)

\section{Inklusion und Sozialraum}

Behindertenrecht und Behindertenpolitik in der Kommune

\section{Nomos}


Die Deutsche Nationalbibliothek verzeichnet diese Publikation in der Deutschen Nationalbibliografie; detaillierte bibliografische Daten sind im Internet über http://dnb.d-nb.de abrufbar.

ISBN 978-3-8487-0688-4

1. Auflage 2013

(c) Nomos Verlagsgesellschaft, Baden-Baden 2013. Printed in Germany. Alle Rechte, auch die des Nachdrucks von Auszügen, der fotomechanischen Wiedergabe und der Übersetzung, vorbehalten. Gedruckt auf alterungsbeständigem Papier. 


\section{Vorwort}

Die Lebenslagen von behinderten Menschen wurden in den letzten zehn Jahren in Deutschland vor allem von drei Faktoren entscheidend geprägt: der International Classification of Functioning, Disability and Health (ICF), dem Neunten Buch Sozialgesetzbuch (SGB IX) und jüngst der UN-Behindertenrechtskonvention (UN-BRK). Immer sind dabei - wenn auch in unterschiedlichen Intensitäten - die Maßgaben der Teilhabe und Selbstbestimmung beziehungsweise die Suche nach (mehr) Handlungsspielräumen und Inklusion wesentlich. Seit Inkrafttreten der UN-BRK in Deutschland im Jahr 2009 wird zudem der Versuch unternommen, die Relativität und Relationalität von Behinderung neu zu reflektieren und die interdependenten Ziele der Autonomie und Inklusion in einem Konzept des „Disability Mainstreaming“ zu vereinen, wie dies die Präambel der UN-BRK nahelegt.

Vor diesem Hintergrund war es das Ziel der am Max-Planck-Institut für Sozialrecht und Sozialpolitik am 18./19. Juni 2012 veranstalteten Fachtagung zu dem Thema „Inklusion und Sozialraum - Behindertenrecht und Behindertenpolitik in der Kommune“, die aktuelle Debatte aufzunehmen und herauszuarbeiten, welchen Herausforderungen die Gestaltung der konkreten Lebenssituation von Menschen mit Behinderungen in der Lebensspanne heute begegnen muss. Dabei konzentrierten sich die Beiträge auf den Sozialraum als Ort im räumlichen wie sozialen Sinn, an dem sich alle Akteure - die Betroffenen, ihre Angehörigen, die Leistungsträger wie Leistungserbringer - begegnen und an dem sie unter Berücksichtigung sowohl des sozialen Umfeldes als auch der lokalen wie der entsprechenden (infra-)strukturellen Besonderheiten zusammenwirken.

Die Fachtagung wollte einen Diskurs von Sozialrecht und Sozialwissenschaften eröffnen und die Möglichkeit bieten, deren unterschiedliche Perspektiven zu verdeutlichen und zu bewerten. Dazu eingeladen wurden Vertreterinnen und Vertreter der Wissenschaft und Praxis. Der damit angestoßene Austausch wird im vorliegenden Tagungsband durch den Abdruck der ein breites thematisches Spektrum umfassenden Beiträge dokumentiert.

Danken möchten wir vor allem den Vortragenden, die uns ihre Beiträge für diesen Tagungsband zur Verfügung gestellt und damit eine Dokumentation der Fachtagung ermöglicht haben. Unser Dank gilt zudem allen Mitarbeiterinnen und Mitarbeitern des Instituts, die mit ihrer Hilfe bei Vorbereitung und Durchführung der Tagung sowie der Fertigstellung des Tagungsbandes einen wesentlichen Anteil an dem Projekt geleistet haben.

München, im April 2013

Ulrich Becker Elisabeth Wacker Minou Banafsche 


\section{Inhaltsverzeichnis}

\section{Grundlagen}

Aufgaben und Handlungsspielräume der Kommunen als Ort der Inklusion Ulrich Becker

Überall und nirgendwo - „Disability Mainstreaming“ im kommunalen Lebensraum und Sozialraumorientierung als Transformationskonzept Elisabeth Wacker

Inklusionsorientierung im Sozialraum - Verpflichtung und Herausforderung Clemens Dannenbeck

II. Barrieren der Inklusion und Perspektiven ihrer Überwindung Inklusionsbarrieren im Sozialraum Markus Dederich

„Mit gleichen Wahlmöglichkeiten in der Gemeinschaft leben“ - Behinderungen und Enthinderungen selbstbestimmter Lebensführung

Gudrun Wansing

Rechtliche Grundlagen einer örtlichen Teilhabeplanung Felix Welti

Barrieren im Sozialraum - ein Kommentar Markus Schäfers

Inklusion im Sozialraum aus Sicht des Deutschen Vereins Andreas Kuhn

\section{Inklusion über die Lebensspanne}

\section{Alter und Behinderung}

Behinderung und Pflegebedürftigkeit im Alter - sind die sozialrechtlichen Reaktionen konsistent?

Gerhard Igl 
Behindertenrecht und Behindertenpolitik in der Kommune ein Blick in die Rechtsprechung

Behindertenrecht und Behindertenpolitik in der Kommune aus kommunaler Sicht

Helmut Kneppe

2. Kinder und Jugendliche mit Behinderungen

Die ,große Lösung“ - eine unendliche Geschichte?

Reinhard Wiesner

Die ,große Lösung“ als gleichheitsrechtliches Gebot -

Verfassungsrechtliche Grenzen des dualen Systems der Eingliederungshilfe für Kinder und Jugendliche

Minou Banafsche

Kinder und Jugendliche mit Behinderungen in der Kommune -

Kommentar aus Sicht eines Jugendamtes

Maria Kurz-Adam

Kinder und Jugendliche mit Behinderungen in der Kommune -

Kommentar aus Sicht einer Schule

Martina Weide-Gertke und Brigitta Seidenschwang

Autorenverzeichnis 
I. Grundlagen 


\title{
Aufgaben und Handlungsspielräume der Kommunen als Ort der Inklusion
}

\author{
Ulrich Becker
}

I. Einleitung 11

II. Kommunale Verantwortung für die Inklusion 13

1. Zuständigkeiten: Von den Kommunen zum Staat und zurück? 13

2. Aufgabenfelder: Kommunale Aufgaben $\quad 15$

a) Sozialrechtliche Aufgaben $\quad 15$

b) Sozialraumorientierung $\quad 17$

c) Quer liegende Direktiven und allgemeine Aufgaben 18

III. Bedingungen kommunalen Handelns in der föderalen Ordnung 19

1. Grenzen der Selbstverwaltung 19

a) Unitarische gesetzliche Vorgaben im Sozialstaat 19

b) Zersplitterung kommunaler Aufgabenwahrnehmung 20

2. Die Finanzierung sozialstaatlicher Aufgaben 21

a) Grundsätze $\quad 21$

b) Aufgabenübertragungsverbot und Konnexitätsprinzip 23

$\begin{array}{ll}\text { IV. Schluß } & 24\end{array}$

\section{Einleitung}

1. Ereignisse haben ihre Zeit, und sie haben ihren Raum. In der bildreichen englischen Sprache heißt es ,events take place“. Diese an sich simple, wenn auch nicht banale Feststellung hat lange in der Sozialpolitik wenig Beachtung gefunden. Deren räumlicher Aspekt spielte kaum eine Rolle. Das hat sich grundlegend geändert, und die Karriere des Begriffs „Sozialraum“1 verdankt sich vielleicht auch dem Umstand, daß er aus weitgehend unbearbeitetem Grund aufgestiegen ist - womit nicht gleichzeitig etwas gegen dessen Bedeutung gesagt ist. Die Notwendigkeit, die räumlichen Bezüge sozialpolitischer Anliegen und Maßnahmen in den Blick zu nehmen, steht vielmehr außer Zweifel.

Welche Analysemöglichkeiten die Raumbezogenheit der Sozialpolitik eröffnet, hängt von dem Raumbegriff ebenso ab wie von der Bedeutung räumlich bedingter Vo-

1 Dazu etwa Jordan, Sozialraumorientierung in der Kinder- und Jugendhilfe, in: Kreft/Mielenz/Trauernicht/Jordan (Hrsg.), Fortschritt durch Recht, Festschrift für Johannes Münder, 2004, S. 255 ff. 
raussetzungen der Interaktion und der personalen Entfaltung. Aus juristischer Perspektive ist der räumliche Bezug aus zwei Gründen aufschlußreich.

Zum einen steht hinter ihm die Ortsgebundenheit hoheitlicher Gewalt: Diese Gewalt ist im Hinblick auf ihren Ausgangspunkt, die Verleihung in Verbindung mit ihrer Legitimation, wie auch im Hinblick auf ihre Wirkung räumlich gegliedert und auf mehrere Ebenen verteilt. Insofern kann von räumlich gestuften Rechtsordnungen gesprochen werden, die zumeist, wenn auch auf der Ebene des Völkerrechts mit (noch) eingeschränkter Bedeutung, ${ }^{2}$ politische Gemeinschaften konstituieren. Die Zuordnung von Kompetenzen zu diesen Ebenen und das Verhältnis des auf der jeweiligen Ebene gesetzten Rechts zueinander sind seit jeher ein klassisches Feld rechtswissenschaftlicher Betätigung.

Zum anderen läßt der Raumbezug zugleich eine alternative, in der Regel dominierende Gliederung in den Hintergrund treten, nämlich die nach sozialpolitischen Sachmaterien. ${ }^{3}$ Das ist deshalb vielfach weiterführend, weil eine Ordnung nach Materien an mehr oder weniger historisch zufällig entstandenen Institutionen hängt und deshalb Gefahr läuft, Zusammenhängendes nicht in den Blick zu nehmen. Diese Gefahr wird heute verstärkt wahrgenommen, ${ }^{4}$ weil sozialpolitische Ziele neu formuliert und übergreifender verwirklicht werden müssen, wofür die Inklusion von behinderten Menschen ein besonders prominentes Beispiel darstellt. Hingegen ist der Raum, auch wenn er keineswegs als feste vorgegebene Größe angesehen werden kann, sondern je nach relevanter Fragestellung differiert und oft auch erst durch die jeweils Handelnden und die dabei relevanten Handlungszusammenhänge geschaffen wird, doch immerhin auch ein von außen beschreibbarer allgemeiner Bezugspunkt.

2. Damit ist über die Begrenzung des Raums noch nichts gesagt. Die Kommune bei unserer Tagung und deshalb auch den folgenden Ausführungen in den Mittelpunkt einer raumbezogenen Betrachtung zu stellen, hat gute Gründe. Lange Zeit weitgehend unbeachtet, gewinnt sie im Zuge neuerer sozialpolitischer Entwicklungen an Bedeutung. Das soll in einem ersten Schritt, zusammen mit den Gründen für diese Aufwertung, kurz

2 Vgl. aber auch Uerpmann, Die Europäische Menschenrechtskonvention und die deutsche Rechtsprechung - Ein Beitrag zum Thema Völkerrecht und Landesrecht, 1993; ders., Internationales Verfassungsrecht, JZ 2001, S. 565 ff.; Pfeffer, Das Verhältnis von Völkerrecht und Landesrecht - Eine kritische Betrachtung alter und neuer Lehren unter besonderer Berücksichtigung der Europäischen Menschenrechtskonvention, 2009.

3 Klassisch und mit internationaler Bedeutung das Abkommen Nr. 102 der IAO vom 28. Juni 1952 über die Mindestnormen der Sozialen Sicherheit (BGB1. II 1957, S. 1321, 1322; abrufbar auch unter http://www.ilo.org/ilolex/german/docs/convdisp1.htm).

4 Vgl. nur Becker, Sozialrecht und Sozialrechtswissenschaft, ZÖR 65 (2010), S. 607, 618 ff.; ders., Das gegliederte System der sozialen Sicherung - bietet die Rechtsvergleichung eine Anleitung zur Vereinfachung?, NDV 2010, S. 510 ff.; vgl. zu den sog. „New Social Risks“ Taylor-Gooby (Hrsg.), New risks, new welfare: the transformation of the European welfare state, 2004; Bonoli, The Politics of the New Social Policies: Providing Coverage against New Social Risks in Mature Welfare States, Policy and Politics 33 (2005), S. 431 ff. 
dargelegt werden (II.). Hintergrund ist der Umstand, daß das Soziale auch in gemeinschaftlichen Näheverhältnissen aufgehoben sein kann und die Kommunen ein flächendeckendes wie universell einsetzbares Netz politischer Gemeinschaften darstellen - im übrigen auch in anderen Ländern, in denen sie dementsprechend ebenfalls sozialpolitische Aufgaben wahrnehmen, etwa die local communities in England für die Zurverfügungstellung von social services. ${ }^{5}$ In Deutschland wird die kommunale Aufgabenwahrnehmung in einen mehrfach geteilten föderalen Rahmen gestellt. Die den Kommunen eingeräumte Selbstverwaltung ist zwar eine rechtliche Garantie für Handlungsoptionen, die aber begrenzt ist: unmittelbar durch gesetzliche Vorgaben, mittelbar und dennoch nicht weniger wirksam durch finanzielle Vorgaben. Beides wird in einem zweiten Schritt behandelt (III.), der als allgemeine Einführung in die rechtlich geformten Bedingungen des kommunalen Handelns dienen soll.

\section{Kommunale Verantwortung für die Inklusion}

\section{Zuständigkeiten: Von den Kommunen zum Staat und zurück?}

Seit der Herausbildung der Städte als eigenen Regeln folgende politische Gemeinschaften - in denen Leibeigenschaft durch Bürgerrechte abgelöst wird, wenn auch nicht ohne Etablierung einer neuen herrschenden Einwohnerschicht - übernahmen diese auch sozialpolitische Aufgaben. Die Errichtung kommunaler Spitäler, die verschiedene Funktionen erfüllten, wofür etwa die Heilig-Geist-Spitäler mit dem berühmten HeiligenGeist-Hospital in Lübeck oder die zum Teil noch heute existierenden Bürgerspitale stehen, aber auch frühe städtische Vorschriften über die Bekämpfung von unbotmäßigem Verhalten ${ }^{6}$ legen davon Zeugnis ab.

Schon mit der Konfessionsspaltung und der ersten Säkularisierungswelle verlagerten sich die Verantwortlichkeiten zur Lösung sozialer Probleme von der gesellschaftlichen hin zur gemeinschaftlichen Sphäre, vor allem im Schul- und Armenwesen. ${ }^{7}$ Mit der Herausbildung der modernen Nationalstaaten erfuhr diese Vergemeinschaftung eine staatliche Überformung. Das preußische ALR bringt das zum Ausdruck, wenn es dort heißt: „Dem Staate kommt es zu, für die Ernährung und Verpflegung derjenigen Bürger zu sorgen, die sich ihren Unterhalt nicht selbst verschaffen, und denselben auch von

5 Vgl. zu Pflegeleistungen näher Landauer, Die staatliche Verantwortung für die stationäre Langzeitpflege in England und Deutschland, 2012, S. $137 \mathrm{ff}$.

6 Das gilt selbst in London, obwohl in den englischen Städten der Königsfriede und nicht ein städtischer Sonderfriede als Grundlage der Ordnung galt, vgl. dazu Rexroth, Das Milieu der Nacht, 1999, S. 67 ff., am Beispiel des Umgangs der Obrigkeit mit Randgruppen im spätmittelalterlichen London.

7 Stolleis, Geschichte des Sozialrechts, in Deutschland, 2003, S. $15 \mathrm{ff}$. 
andern Privatpersonen, welche nach besonderen Gesetzen dazu verpflichtet sind, nicht erhalten können. " 8 Zwar bleiben die Kommunen hier ebenfalls verpflichtet. ${ }^{9}$ Und die Verpflichtung wird inhaltsreicher: So spricht Hegel in seinen Zusätzen zu den Grundlinien der Philosophie des Rechts, ohne jemals die Gemeinden zu erwähnen, von einer „Polizei“ und meint damit die Schaffung einer lebensbefähigenden Ordnung der bürgerlichen Gesellschaft, deren Inhalte sehr an den viel später von Forsthoff entwickelten und umfassenderen Begriff der Daseinsvorsorge ${ }^{10}$ erinnern. Denn Hegel führt aus: „Die polizeiliche Aufsicht und Vorsorge hat den Zweck, das Individuum mit der allgemeinen Möglichkeit zu vermitteln, die zur Erreichung der individuellen Zwecke vorhanden ist. Sie hat für Straßenbeleuchtung, Brückenbau, Taxation der täglichen Bedürfnisse, so wie für die Gesundheit Sorge zu tragen."11

Jedoch stehen die Kommunen nun unter einer staatlichen Gesamtverantwortung. Damit und mit der nachfolgenden Konstitutionalisierung zunächst auf Landes-, dann auf Bundesebene waren die Grundsteine für eine umfassende staatliche Sozialpolitik gelegt, die im 20. Jahrhundert näher ausformuliert wurde.

Vor diesem Hintergrund ist bemerkenswert, daß seit einigen Jahren von einer „Rekommunalisierung“ die Rede ist - zwar zum Teil bezogen auf die Rückgängigmachung von Privatisierungen der Daseinsvorsorge, 12 zum Teil aber auch auf die Gewährung von Sozialleistungen. Diese Entwicklung hat ihren Grund in veränderten sozialpolitischen Zielsetzungen, nämlich dem Versuch, stärker auf das Verhalten Einzelner Einfluß zu nehmen. Für diese je nach Standpunkt pädagogischen oder paternalistischen Bestrebungen ${ }^{13}$ stehen die Aktivierungsstrategie, aber auch die immer weiter verfeinerte Bekämpfung von Diskriminierungen. Nun kann man sich darüber streiten, inwieweit sozialpolitische Interventionen darauf gerichtet sein müssen oder dürfen, Teilhabechancen zu eröffnen oder tatsächlich eine Teilhabe zu bewirken. In jedem Fall spielen aber für teilhabebezogene Ansätze soziale Interaktionen im unmittelbaren Umfeld, in räumlicher Nähe, eine besondere Rolle. Die selbstbestimmte Entfaltung wie der Zugang zu existentiell wichtigen Leistungen und Einrichtungen sind nur in konkreten räumlichen Zusammenhängen möglich. Daß Kommunen insofern eine besondere Verantwortung und Gestaltungsaufgabe zukommt, liegt auf der Hand. Sie können aufgrund ihrer

8 19. Titel, § 1, zitiert nach Koch, Allgemeines Landrecht für die preußischen Staaten, Band 4,1, 3. Aufl. 1863.

9 19. Titel, § 10: „Auch Stadt- und Dorfgemeinden müssen für die Ernährung ihrer verarmten Mitglieder und Einwohner sorgen.“

10 Forsthoff, Die Verwaltung als Leistungsträger, 1938; ders., Rechtsfragen der leistenden Verwaltung, 1959; zu der Begriffsentfaltung Kersten, Die Entwicklung des Konzepts der Daseinsvorsorge im Werk von Ernst Forsthoff, Der Staat 44 (2005), S. 543 ff.; zu dessen heutiger Bedeutung Krajewski, Rechtsbegriff Daseinsvorsorge?, VerwArch 99 (2008), S. 174 ff.

11 Hegel, Grundlinien der Philosophie des Rechts, in: ders., Sämtliche Werke. Jubiläumsausgabe in zwanzig Bänden. Siebenter Band, hrsg. von Glockner, 4. Aufl. 1964, Zusatz zu § 236.

12 Dazu Bauer, Zukunftsthema „Rekommunalisierung“, DÖV 2012, S. 329 ff.

13 Vgl. Heinig, Paternalismus im Sozialstaat, in: Anderheiden/Bürkli/Heinig/Kirste/Seelmann (Hrsg.), Paternalismus und Recht, 2006, S. 157 ff. 
Kenntnisse der örtlichen Gegebenheiten in vielen Fällen zur zunehmend angestrebten Zielgerichtetheit sozialer Leistungen beitragen. Und sie bilden ein flächendeckendes Netz erreichbarer Behörden, das eine umfassende räumliche Abdeckung der Bemühungen um sozialen Schutz aller Einwohner erleichtert.

Das bedeutet nicht ein Zurück zu mittelalterlichen Verhältnissen - auch wenn etwa das Fördern und Fordern, wie es der im SGB II angelegten Aktivierung zugrunde liegt, an eine obrigkeitliche Armenpolitik im Zeichen von „Zuwendung und Strenge“, wie das Stolleis formuliert hat, ${ }^{14}$ erinnert. Die rechtlichen Rahmenbedingungen der kommunalen Sozialpolitik haben sich vielmehr in zweifacher Weise grundlegend geändert. Erstens beruht diese, bei aller Wiederentdeckung von Eigenverantwortung und der damit auch verbundenen Vorstellung, die Gemeinschaften funktionsfähig zu halten, ${ }^{15}$ auf einer menschenrechtlichen Basis: Jeder Einzelne besitzt durchsetzbare Ansprüche auf sozialen Schutz, die nicht von einem sittlichen Wohlverhalten, sondern höchstens von gesetzlich festgelegten und mit dem Schutzzweck vereinbaren Voraussetzungen abhängen. Zweitens sind die Kommunen in eine gesamtstaatliche und zunehmend sogar EUweite Verantwortung eingebunden, ${ }^{16}$ die ihnen rechtlich verbindliche Vorgaben auferlegt und zu einer zunehmenden rechtlichen Verflechtung zwischen den politischen Ebenen führt.

\section{Aufgabenfelder: Kommunale Aufgaben}

\section{a) Sozialrechtliche Aufgaben}

Bevor auf diese Verflechtung zurückzukommen ist, soll ein Blick auf die für die Themenstellung relevanten kommunalen Aufgaben und ihre jüngsten Veränderungen geworfen werden, beginnend mit denen, die aus dem formalen Sozialrecht abzuleiten sind. In diesem Zusammenhang läßt sich die These von der wachsenden sozialpolitischen Bedeutung der Kommunen sowohl in quantitativer als auch in qualitativer Hinsicht belegen, wenn auch hier nur in Stichworten.

14 Stolleis, Geschichte des Sozialrechts (Fußn. 7), S. 17.

15 Becker, Sozialmodell und Menschenbild in der „Hartz-IV” Gesetzgebung, in: Behrends/Schumann (Hrsg.), Gesetzgebung, Menschenbild und Sozialmodell im Familien- und Sozialrecht, Abhandlungen der Akademie der Wissenschaften zu Göttingen, 2008, S. 39 ff.; grds. Zacher, Grundtypen des Sozialrechts, in: Fürst/Herzog/Umbach (Hrsg.), FS für Zeidler, 1987, S. 571, 572.

16 Vgl. Art. 4 Abs. 2 S. 1 EUV; Art. 5 Abs. 3 EUV; Art. 170 Abs. 1 AEUV; Art. 300 AEUV; Protokoll Nr. 26 des AEUV über Dienste von allgemeinem Interesse. Aus dem Schrifttum: Knauff, Die Kommunen als Träger der Daseinsvorsorge, WiVerw 2011, S. 79 ff.; Schmidt-Leithoff, Gemeindewirtschaft im Wettbewerb, 2011; Burgi, Kommunalrecht, 3. Aufl. 2010, § 4 Kommunen und Europarecht; Pfisterer, Kommunale Selbstverwaltung und lokale Governance vor dem Hintergrund des europäischen Integrationsprozesses, 2009; Bergmann, Deutsche Kommunen und Europa, BWGZ 2009, S. $300 \mathrm{ff}$. 
aa) Mit dem verstärkten Bekenntnis zu Inklusion und Teilhabe von Menschen mit Behinderung sind neue Querschnittsaufgaben entstanden, und das nicht erst durch Inkrafttreten des SGB IX im Jahre 2001. Zu nennen sind hier die Eingliederungshilfe für behinderte Menschen gem. $\S \S 53 \mathrm{ff}$. SGB XII sowie die Eingliederungshilfe für seelisch behinderte Kinder und Jugendliche gem. § 35a SGB VIII ${ }^{17}$, wobei es dem Gesetzgeber bislang nicht gelungen ist, die Schwierigkeiten, die hinsichtlich der Abgrenzung dieser beiden Formen von Hilfe für junge Menschen mit Behinderung entstehen, zu beseitigen. ${ }^{18}$

Auch die bereits angesprochene aktivierende Arbeitsmarktpolitik bringt hinsichtlich der Eingliederung von Menschen mit Behinderung neue Aufgaben für die Kommunen. Sie ist schon nach $\S 1$ Abs. 2 S. 3 Nr. 5 SGB II auf die Überwindung von behindertenspezifischen Nachteilen auszurichten, was bei den Eingliederungsleistungen gem. § 16 Abs. 1 S. 2 SGB II zu beachten ist. Dabei fordert nun § 16a SGB II ${ }^{19}$, zur Verwirklichung einer ganzheitlichen und umfassenden Betreuung und Unterstützung bei der Eingliederung in Arbeit auch die Betreuung minderjähriger oder behinderter Kinder oder die häusliche Pflege von Angehörigen vorzusehen. Zudem wird gem. § 21 Abs. 4 S. 1 SGB II ein Mehrbedarf von erwerbsfähigen behinderten Menschen im Rahmen der Grundsicherung anerkannt.

bb) Gleichzeitig sind die Anforderungen an bereits bestehende Aufgaben weiter erhöht worden, was sich besonders gut in der Kinder- und Jugendhilfe beobachten läßt, einer Kernmaterie kommunaler Sozialpolitik, die nach Lorenz von Stein in „der polizeilichen Epoche“ als „Sorge der Verwaltung für das Leben der geborenen Kinder“ zu einer öffentlichen Aufgabe wurde. ${ }^{20}$

Seit Inkrafttreten des SGB VIII im Jahre 1990 gab es zahlreiche Änderungen, die unter Kindesschutz-21, aber auch Qualitätsaspekten ${ }^{22}$ die Aufgabenerfüllung anspruchs-

17 Jüngst zur Begriffsproblematik der „seelischen Behinderung“ i.S.v. § 35a SGB VIII Riehle, Anmerkungen zu § 35a SGB VIII, ZFSH/SGB 2012, S. 207 ff.

18 Dazu Pitschas, Behinderte Menschen in der kommunalen Sozialpolitik, SGb 2009, S. 253, 259 f.; Banafsche, Kinder und Jugendliche mit Behinderung zwischen SGB VIII und SGB XII - im Lichte der UN-Behindertenrechtskonvention, ZKJ 2011, S. 116 ff.; Gerlach/Hinrichs, Inklusion und die „Große Lösung“ für die Jugend- und Behindertenhilfe, ZKJ 2012, S. 86 ff. (Teil 1), 130 ff. (Teil 2).

19 Eingefügt mit Wirkung zum 1.1.2009 durch das Gesetz zur Neuausrichtung der arbeitsmarktpolitischen Instrumente (ArbMINAG) v. 21.12.2008, BGB1. I 2008, S. 2859.

20 Lorenz v. Stein, Die Verwaltungslehre, Teil 2: Wirkliche Innere Verwaltung, 2. Neudruck der Ausgabe Stuttgart 1866, 1975, S. 162.

21 Etwa durch Einfügung des $\S 8$ a in das SGB VIII mit Wirkung zum 1.10.2005 durch das Gesetz zur Weiterentwicklung der Kinder- und Jugendhilfe - KICK: Kinder- und Jugendhilfeweiterentwicklungsgesetz, verkündet am 27.12.2004, BGBl. I 2004, S. 3852., der bei Anzeichen einer Kindeswohlgefährdung umfassende Mitteilungs- und Dokumentationspflichten des Jugendamtes aber auch der freien Träger vorsieht oder durch Inkrafttreten des Gesetzes zur Stärkung eines aktiven Schutzes von Kindern und Jugendlichen (Bundeskinderschutzgesetz - BKiSchG) v. 22.12.2011 (BGBl. I 2011, S. 2975), das unter anderem zur Sicherstellung von persönlicher Zuverlässigkeit sämtlicher in der Jugendhilfe tätigen Personen inklusive Ehrenamtlicher die Vorlage eines Führungszeugnisses 
voller werden lassen. Kommunen als Leistungsträger sind im Rahmen ihrer als Gesamtverantwortung (§ 79 SGB VIII) bezeichneten Gewährleistungsverantwortung bei der Aufsicht und Steuerung freier Träger mit höheren Anforderungen konfrontiert. Hinzuweisen ist neben der Planungsverantwortung ( $\$ 80$ SGB VIII) auf die durch das BKiSchG neu eingefügte Aufgabe der Qualitätsentwicklung (§ 79a SGB VIII). Ferner bringt der mit dem Kinderförderungsgesetz (KiföG) ${ }^{23}$ in Gang gesetzte Ausbau institutionalisierter Kinderbetreuung 24 neue Herausforderungen für die Kommune, und zwar sowohl in deren Rolle als Leistungsträger, weil die neuen entstehenden Kindertageseinrichtungen auch beaufsichtigt werden wollen ${ }^{25}$, aber auch als Leistungserbringer, weil Kitas zu einem großen Teil kommunale Einrichtungen sind - und demnächst, d.h. ab dem 1.8.2013, werden sich die Kommunen den Rechtsansprüchen auf Kinderkrippenplätze ausgesetzt sehen.

\section{b) Sozialraumorientierung}

Die erwähnte Planungsverantwortung in der Jugendhilfe enthält zugleich einen der wenigen normativen Anknüpfungspunkte für eine Sozialraumorientierung kommunaler Sozialpolitik. Er kommt zum Ausdruck durch die Formulierung „Einrichtungen und Dienste sollen so geplant werden, daß insbesondere Kontakte [...] im sozialen Umfeld gepflegt und erhalten werden können“ ( 88 Abs. 2 Nr. 1 SGB VIII). Ähnliche Bezugnahmen finden sich in den Einleitungsvorschriften des SGB VIII, nämlich auf die „Umwelt“ (§ 1 Abs. 3 S. 4 SGB VIII), sowie im Zusammenhang mit den Hilfen zur Erziehung, die das „soziale Umfeld des Kindes oder des Jugendlichen" einbeziehen sollen (§ 27 Abs. 2 S. 2 Hs. 2 SGB VIII).

Die rechtliche Bedeutung dieser räumlichen Orientierung bleibt allerdings fraglich. ${ }^{26}$ Sie enthält nach der Rechtsprechung und dem Schrifttum jedenfalls keine Rechtsgrundlage für eine budgetierende Finanzierung von Leistungserbringern. ${ }^{27}$ Eine klare gesetz-

gem. $\S \S 30,30$ a BZRG beim jeweiligen Träger der öffentlichen Jugendhilfe in regelmäßigen Abständen vorsieht, vgl. dazu Weber, Das erweiterte Führungszeugnis als Instrument des Kinderschutzes, JAmt 2012, S. 62 ff.

22 Etwa durch mit Einführung der $\S \S 78 \mathrm{a}$ ff. SGB VIII durch das Zweite Gesetz zur Änderung des Elften Buches Sozialgesetzbuch und anderer Gesetze vom 29.5.1998, BGBl. I 1998, S. 1188; vgl. allgemein zu Qualitätsaspekten im Sozialrecht die Beiträge in SDSRV 61 (2012) sowie Becker/Meeßen/Neueder/Schlegelmilch/Schön/Vilaclara, Strukturen und Prinzipien der Leistungserbringung im Sozialrecht - Teil 2, VSSR 2012, S. 1, 21 ff.

23 Gesetz zur Förderung von Kindern unter drei Jahren in Tageseinrichtungen und in Kindertagespflege (Kinderförderungsgesetz - KiföG), BGBl. I 2008, S. 2403.

24 Vgl. 7. Familienbericht der Bundesregierung, BT-Drucks. 16/1360, S. 54 ff., zu den infrastrukturellen Komponenten einer zeitgemäßen Familienpolitik.

25 So schreibt $\S 45$ SGB VIII auch eine Betriebserlaubnis für Kitas vor.

26 Dazu Nellissen, Sozialraumorientierung im aktivierenden Sozialstaat, 2006, S. $22 \mathrm{ff}$.

27 So jüngst wieder OVG Nieders. v. 9.7.2010, 4 ME 306/09 = JAmt 2011, 102; dazu Münder, Wieder einmal: Sozialraumorientierung auf dem rechtlichen Prüfstand, JAmt 2011, S. 69 ff.; ebenso Wall- 
liche Grundlage wäre aber angesichts des Grundrechts der Berufsfreiheit der Leistungserbringer ${ }^{28}$ sowie des Wunsch- und Wahlrechts der Leistungsempfänger ${ }^{29}$ erforderlich, um die mit einer entsprechenden Finanzierung bezweckte Exklusivität zu rechtfertigen.

Das Sozialraumkonzept hat insofern keine unmittelbare Außenwirkung. Es führt zu nicht mehr, aber auch nicht weniger als einer Verpflichtung, das Handeln sämtlicher sozialer Akteure auch auf der lokalen Ebene unter sozialpolitischer Perspektive miteinander abzustimmen. Das immerhin bringt für die Kommunen im Rahmen ihrer Planungsverantwortung eine neue und nicht zu unterschätzende Vernetzungsaufgabe mit sich. ${ }^{30}$

\section{c) Quer liegende Direktiven und allgemeine Aufgaben}

Nicht zuletzt weil die Inklusion behinderter Menschen auf verfassungsrechtlichen und vor allem mit der UN-Konvention über die Rechte von Menschen mit Behinderungen $^{31}$ auch auf völkerrechtlichen Direktiven beruht ${ }^{32}$, die noch im einzelnen Gegenstand der nachfolgenden Beiträge sein werden, ist der Blick über das Sozialrecht ${ }^{33}$ hinaus zu weiten.

Zum einen auf andere und eigenständig geregelte Politikbereiche, insbesondere das Schulrecht, das hier zumindest einmal genannt werden soll. Die nie verstummte „Integrationsdebatte“34 ist hier ebenfalls als „Inklusionsdebatte“ neu und stärker als je zuvor entbrannt, was auch in dieser Hinsicht den bemerkenswert starken Einfluß der genann-

rabenstein, Wettbewerb in der Sozialwirtschaft: rechtliche Steuerung der politischen Steuerung, Archiv für Wissenschaft und Praxis der Sozialen Arbeit 2009, S. 36, 41.

28 Zur Berufsfreiheit der Leistungserbringer Becker/Meeßen/Neueder/Schlegelmilch/Schön/Vilaclara, Strukturen und Prinzipien der Leistungserbringung im Sozialrecht - Teil 1, VSSR 2011, S. 323, 343 ff.

29 Zum Individualisierungsprinzip Becker/Meeßen/Neueder/Schlegelmilch/Schön/Vilaclara, Strukturen und Prinzipien der Leistungserbringung im Sozialrecht - Teil 3, VSSR 2012, S. 103, $121 \mathrm{ff}$.

30 Vgl. bspw. das Konzept „REGSAM - Regionale Netzwerke für Soziale Arbeit“ der Landeshauptstadt München, das Rahmenbedingungen für eine Vernetzung von Politik, Verwaltung, Bürgerinnen und Bürgern, Verbänden und sonstigen Akteuren sozialer Arbeit setzt. Informationen abrufbar unter http://www.regsam.net/de. Allgemein zu kommunaler Sozialpolitik als Querschnittsaufgabe und Netzwerkstruktur Pitschas, Kommunale Sozialpolitik, in: v. Maydell/Ruland (Hrsg.), Sozialrechtshandbuch (SRH), 3. Auflage 2003, S. 1295, 1333, Rdnr. 96 ff.

31 Überführung in deutsches Bundesrecht durch das „Gesetz zu dem Übereinkommen der Vereinten Nationen vom 13.12.2006 über die Rechte von Menschen mit Behinderungen sowie zu dem Fakultativprotokoll vom 13.12.2006 zum Übereinkommen der Vereinten Nationen über die Rechte von Menschen mit Behinderungen“"vom 21.12.2008 (BGB1. II 2008, S. 1419).

32 Dazu einführend Welti, Rechtliche Voraussetzungen von Barrierefreiheit, NVwZ 2012, S. 725 ff.

33 Zur Vereinbarkeit der UN-Behindertenrechtskonvention mit dem deutschen Sozialrecht jüngst $\mathrm{Ba}$ nafsche, Die UN-Behindertenrechtskonvention und das deutsche Sozialrecht, SGb 2012, S. $373 \mathrm{ff}$. (Teil I) und S. $440 \mathrm{ff}$. (Teil II).

34 Vgl. nur Becker/Graser (Hrsg.), Perspektiven der schulischen Integration von Kindern mit Behinderung, 2004. 
ten UN-Behindertenrechtskonvention belegt. Damit sind so viele und vielfältige Fragen aufgeworfen, daß diese einer eigenständigen Behandlung bedürfen und deshalb nicht Gegenstand dieser Tagung sein sollen, auch wenn sie kommunale Schulen betreffen.

Zum anderen und viel allgemeiner kann sich auch im übrigen die angestrebte Inklusion nicht in der Zurverfügungstellung spezieller Leistungen im Sinne eines Ausgleichs spezifischer Nachteile erschöpfen, sondern stellt neben einer Förderung zugleich Anforderungen an die Öffnung der für die Allgemeinheit gedachten Einrichtungen. Das beginnt bei der Benutzung der Straßen und öffentlichen Verkehrsmittel, geht über die Museen, Sportanlagen und Theater bis zur Ermöglichung der Kommunikation. Dabei unterliegt die kommunale Aufgabenerfüllung quer liegenden, auf bestimmte Tätigkeitsfelder bezogenen Konkretisierungen der allgemeinen Vorgaben. Das gilt insbesondere für das Baurecht: Zu nennen sind hier § 171e BauGB, der unter dem Titel „soziale Stadt“ Maßnahmen zur Verhinderung von Segregation vorsieht, die allerdings Einzelpersonen nicht unmittelbar zugute kommen, und $\S 1$ Abs. 6 Nr. 3 BauGB, wonach die Bedürfnisse von Menschen mit Behinderung als öffentlicher Belang im Rahmen der Bauleitplanung zu beachten sind. Praktisch noch wichtiger sind die landesrechtlichen Vorschriften zum barrierefreien Bauen wie etwa Art. 48 BayBO. Dessen Abs. 2 betrifft öffentlich zugängliche Anlagen, neben den gemeindlichen Einrichtungen im eigentlichen Sinn gehören dazu auch Verwaltungsgebäude und private Einrichtungen wie Hotels und Gaststätten. Er wird in Bayern - wie in anderen Ländern ${ }^{35}$ - flankiert durch eine gleichstellungspolitische Vorschrift (Art. 10 Abs. 1 S. 1, 2 des Bayerischen Behindertengleichstellungsgesetzes - BayBBG), nach der die Träger öffentlicher Gewalt ihre Neubauten sowie große Um- oder Erweiterungsbauten entsprechend den allgemein anerkannten Regeln der Technik barrierefrei zu gestalten haben. Diese Verpflichtung steht - anders als die des Bauordnungsrechts - nicht unter dem Vorbehalt einer verhältnismäßigen Mehrbelastung.

\section{Bedingungen kommunalen Handelns in der föderalen Ordnung}

\section{Grenzen der Selbstverwaltung}

\section{a) Unitarische gesetzliche Vorgaben im Sozialstaat}

Kommunen sind also sowohl als Sozialleistungsträger wie als Garanten einer weit verstandenen, kulturelle und sportliche Einrichtungen mit umfassenden Daseinsvorsorge gefordert, wenn es um die Inklusion von Menschen mit Behinderungen geht. Grund-

35 Vgl. zu den Texten die Sammlung von Frehe/Welti (Hrsg.), Behindertengleichstellungsrecht - Textsammlung mit Einführungen, 2010. 
sätzlich können sie sich bei der Wahrnehmung aller Aufgaben auf die ihnen sowohl durch das Grundgesetz (Art. 28 Abs. 2) wie durch die Landesverfassungen (etwa Art. 11 Abs. 2 BV) eingeräumte Selbstverwaltungsgarantie stützen. Sie dürfen diese Aufgaben eigenverantwortlich wahrnehmen.

Allerdings gilt dafür eine wichtige Einschränkung: Die Selbstverwaltung besteht nur im Rahmen der gesetzlichen Vorgaben. Zwar müssen diese wiederum verfassungsgemäß sein und auch die Rechtsstellung der Kommunen respektieren. Ein unbedingter rechtlicher Schutz wird dadurch aber nur für den Kernbereich der kommunalen Selbstverwaltung vermittelt. ${ }^{36}$

Tatsächlich ist jedenfalls die Freiheit der Kommunen, zu erledigende Aufgaben auszusuchen, weitgehend beschränkt. Viele sozialstaatliche Aufgaben im eigenen Wirkungskreis sind Pflichtaufgaben. Andere Aufgaben werden ihnen durch den Staat übertragen. Insbesondere die Sozialgesetzbücher setzen für die Gewährung von Sozialleistungen auch enge inhaltliche, also das „Wie“ der Aufgabenwahrnehmung betreffende gesetzliche Vorgaben. Der Gesetzgeber mißtraut, ob zu Recht oder Unrecht, zu großen Gestaltungsspielräumen und setzt zumeist auf einheitliche Standards. Zwar folgt aus der Selbstverwaltungsgarantie, daß Kommunen Spielräume zur freien Gestaltung innerhalb der Aufgaben des eigenen Wirkungskreises verbleiben müssen. ${ }^{37}$ Jedoch sind die Ausgaben für freiwillige Selbstverwaltungsaufgaben nach groben Schätzungen von ca. $20 \%$ zu Beginn der 1960er Jahre, über $10 \%$ in den 1970er Jahren auf ca. $5 \%$ zur Jahrtausendwende hin zurückgegangen. ${ }^{38}$

\section{b) Zersplitterung kommunaler Aufgabenwahrnehmung}

Der kommunalen Selbstverwaltung ist eine weitere Begrenzung immanent, nämlich eine räumliche. Auch Kommunen haben Hoheitsgebiete, ihr Handeln ist grundsätzlich in dessen Wirkung auf diese Gebiete beschränkt. Das bedeutet zunächst, daß Selbstverwaltungsspielräume zu Unterschieden in der Ausgestaltung der Inklusion von einer Kommune zur nächsten führen können. Diese territorialen Ungleichbehandlungen sind durch den Gleichheitssatz nicht aufzufangen. ${ }^{39}$ Sie haben aber auf einer vergleichbaren räumlichen Ebene, also zwischen einzelnen Gemeinden, nur eine eingeschränkte praktische Bedeutung. Denn die eben genannten bundesgesetzlichen Vorgaben wirken insofern unitarisierend.

Wichtiger ist deshalb der Umstand, daß jedenfalls die deutschen Flächenstaaten über mehrere, wenn auch unterschiedlich ausgebaute kommunale Ebenen verfügen und die jeweilige Beschränkung der kommunalen Hoheitsgewalt im Zusammenspiel mit der

36 Grundl. BVerfGE 79, 127, 146 ff. (Rastede).

37 Tettinger/Schwarz, in: v. Mangoldt/Klein/Starck (Hrsg.), GG, Bd. 2, 6. Aufl. 2010, Art. 28 Rdnr. 247 ff. m.w.N.

38 Lohse, Kommunale Aufgaben, kommunaler Finanzausgleich und Konnexitätsprinzip, 2005, S. 52 f.

39 Henkel, Die Kommunalisierung von Staatsaufgaben, 2010, S. 212 ff. m.w.N. 
Selbstverwaltung verhindern, daß zwischen diesen Ebenen eine hierarchische Gliederung besteht. Einfacher ausgedrückt kann der überörtliche Träger den örtlichen Träger nicht verwaltungsintern anleiten und lenken. ${ }^{40} \mathrm{Da}$ aber gerade in den Materien der kommunalen Sozialpolitik zwei kommunale Ebenen die Verwaltungszuständigkeiten untereinander aufteilen, kommt es zu Koordinierungsproblemen.

So sind in Bayern (gem. Art. 82 Abs. 1 BayAGSG i.V.m. $§ 3$ Abs. 3 SGB VIII) die Bezirke als überörtliche Träger der Sozialhilfe im eigenen Wirkungskreis für die Eingliederungshilfe für behinderte Menschen - immerhin seit 2008 in vollem Umfang ${ }^{41}-$, für alle übrigen Leistungen der Sozialhilfe, die in stationären bzw. teilstationären Einrichtungen gewährt werden, sowie für die Blindenhilfe gem. § 72 SGB XII zuständig, während Landkreise und kreisfreie Gemeinden als örtliche Träger der Jugendhilfe (gem. Art. 15 Abs. 1 S. 1 BayAGSG) zuständig sind für die erwähnten Leistungen nach § 35a SGB VIII. Auch wenn sich die Bezirke (gem. Art. 51 Abs. 1 S. 1 BayAGSG) an den Kosten der Eingliederungshilfe für seelisch behinderte Kinder und Jugendliche zu beteiligen haben, führt diese Regelung in der Praxis zu zahlreichen Kostenstreitigkeiten zwischen Bezirken und örtlichen Jugendhilfeträgern.

Von solchen Aufgabenüberschneidungen und Schnittstellenproblemen - zu denen auch die notwendige Abstimmung mit anderen Behörden, insbesondere den Pflegekassen im Hinblick auf die Versorgung von pflegebedürftigen Menschen, zählt - wird im Laufe der Tagung sicher noch mehrfach die Rede sein. Denn die Sozialraumperspektive macht, darin liegt gerade ein Vorteil, die aus einem Nebeneinander verschiedener Maßnahmen und zuständiger Träger folgenden Schwierigkeiten sichtbar und drängt auf deren Lösung. Die Kommunen sind sich dessen zunehmend bewußt. Erst jüngst hat darauf und auf ein von ihm initiiertes Modellprojekt beispielsweise der Bezirk Oberbayern in seinem ersten Bericht über eine strukturelle Gesamtplanung hingewiesen. ${ }^{42}$

\section{Die Finanzierung sozialstaatlicher Aufgaben}

\section{a) Grundsätze}

Die Erfüllung sozialpolitischer Aufgaben kostet Geld. Sie ist für die Kommunen mit steigenden Lasten verbunden, was angesichts der genannten Entwicklungen nicht ver-

40 Vgl. nur Becker, in: ders./Heckmann/Kempen/Manssen, Öffentliches Recht in Bayern, 5. Aufl. 2011, S. 76, Rdnr. 16.

41 Mit Inkrafttreten des Zweiten Gesetzes zur Änderung des Gesetzes zur Ausführung der Sozialgesetze vom 20.12.2007, GVB1., S. 979 zum 1.1.2008.

42 Erster Sozialbericht des Bezirks Oberbayern, abrufbar unter http://www.bezirk-oberbayern.de/media/custom/379_3575_1.PDF?1291866617; Wissel/Rohrmann, Örtliche Angebots- und Teilhabeplanung im Landkreis Weilheim-Schongau - Analysen, Einschätzungen, und Empfehlungen des Zentrums für Planung und Evaluation Sozialer Dienste der Universität Siegen, 2010, abrufbar auf http://www.uni-siegen.de/zpe/projekte/teilhabeplanung-wm/pdf/thp_wm_26112010.pdf. 
wundert. Um nur einige Zahlen zu nennen: Die Ausgaben für die Kinder- und Jugendhilfe stiegen bundesweit von 17,1 Mrd. $€$ im Jahr 2001 auf 24,3 Mrd. $€$ im Jahr 2009; die für die Eingliederungshilfe für behinderte Menschen (6. Kapitel SGB XII) von 10,9 Mrd. $€$ im Jahr 2003 auf 13,8 Mrd. $€$ im Jahr 2010.43 Aber auch prozentual nehmen die Ausgaben für Sozialleistungen und soziale Einrichtungen wie Sozialhilfe, Kindergärten, Jugendhilfe, Alten-, Pflege- und Behinderteneinrichtungen einen wachsenden Anteil ein. Beliefen sie sich z.B. bei den Kommunen in Baden-Württemberg im Jahr 1980 noch auf $16 \%$ der Gesamtausgaben, so waren es im Jahr 1990 bereits $22 \%$ und im Jahr 2007 dann $30 \% .44$ Die Aufwendungen für die Ermöglichung eines barrierefreien $\mathrm{Zu}$ gangs zu kommunalen Einrichtungen sind in diesen Zahlen natürlich noch nicht berücksichtigt.

Kommunen besitzen zwar mit eigenen Steuern, vor allem der Grund- und Gewerbesteuer, ihren Anteilen an staatlichen Steuern sowie den Beitrags- und Gebühreneinnahmen eigene Einnahmequellen, bleiben aber zu einem großen Teil auf staatliche Finanzmittel angewiesen. Das wichtigste Instrument zur Verteilung der Mittel stellen die kommunalen Finanzausgleiche dar. Mit ihnen wird Geld aus Steuerverbünden und dem Landeshaushalt auf die Kommunen, aber im Wege der interkommunalen Umlagen auch zwischen kommunalen Ebenen verteilt, wobei neben dem fiskalischen Ziel der Sicherung finanzieller Leistungsfähigkeit Ausgleichseffekte erzielt werden sollen.

$\mathrm{Da}$ trotz dieses Ausgleichs durch die zunehmenden Aufgaben im Zusammenhang mit der Gewährung von Sozialleistungen die Leistungsfähigkeit der Kommunen gefährdet werden kann, ist ein häufig vorgebrachtes Argument. Auf Bundesebene wurde auch deshalb im Jahr 1994 Art. 28 Abs. 2 GG um einen S. 3, Hs. 1 ergänzt. ${ }^{45}$ Danach umfaßt die kommunale Selbstverwaltungsgarantie ,die Grundlagen der finanziellen Eigenverantwortung“. Damit wird das Erfordernis einer angemessenen Finanzausstattung46, das in der Rechtsprechung vieler Verfassungsgerichte der Länder anerkannt ist, ${ }^{47} \mathrm{im}$ Grundgesetz ausdrücklich verankert. Die Bestimmung verleiht den Kommunen aber keine praktisch handhabbare Abwehrposition. ${ }^{48}$ Sie haben wegen der Zweistufigkeit der Finanzverfassung keine unmittelbaren Ansprüche gegen den Bund, Sonderfälle wie den Belastungsausgleich des SGB II ausgenommen.

43 Quelle jeweils Statistisches Bundesamt, https://www.destatis.de (abgerufen am 13.6.2012).

44 Finanzministerium Baden-Württemberg (Hrsg.), Die Gemeinden und ihre Finanzen, 2010, S. 7.

45 42. Gesetz zur Änderung des GG v. 27.10.1994 (BGBl. I 1994, S. 3146).

46 Dazu nur BVerwGE 106, 280, 287. Vgl. auch den Überblick bei Laier, Der kommunale Finanzausgleich, 2008, S. 65 f.

47 Vgl. etwa StGHBW, ESVGH 49, 242; BayVGH, BayVBl. 2008, 172, 175 f.; LVerfG M-V E 17, 297, 318; Nds. StGH E 3, 136, 164; VerfGH NRW, NWVB1. 2008, 223 ff.; VerfGH Rhld-Pf, AS 29, 75, 82; VGH Saarl, AS RP-SL 34, 1 ff.; ThürVGRspr 2005, 233. Vgl. zur Berücksichtigung von Aufgabenerweiterungen VerfGH NRW, OVGE 43, 216.

48 Rennert, Die verfassungsrechtliche Stellung der Gemeinden bei der Zuweisung überörtlicher Aufgaben durch Bundesgesetz, VerwArch 94 (2003), S. 459, 468 ff. 


\section{b) Aufgabenübertragungsverbot und Konnexitätsprinzip}

Daran hat sich durch die Föderalismusreform I nichts geändert. Trotz vorangehender Diskussion wurde ein allgemeiner finanzieller Durchgriff von Bund zu Kommunen weiterhin abgelehnt und statt dessen ein Schutz der Kommunen durch ein Aufgabenübertragungsverbot (Art. 84 Abs. 1 S. 7 GG) eingeführt. Jetzt heißt es im Grundgesetz: „Durch Bundesgesetz dürfen Gemeinden und Gemeindeverbänden Aufgaben nicht übertragen werden." So klar das klingt, so unklar ist die Handhabung. Wichtig wäre das Verbot in erster Linie für Aufgabenänderungen. Ob es diese überhaupt erfaßt, ist umstritten. ${ }^{49}$ Das BVerfG hat dazu noch nie entschieden, wenn es das vielleicht auch demnächst muß. Greift der Schutz, so die Idee, dann müssen die Länder die Aufgabenübertragung vornehmen und dafür nach dem mittlerweile in allen Landesverfassungen vorgesehenen Konnexitätsgrundsatz ${ }^{50}$ einstehen: Denn danach soll zahlen, wer anschafft. Auch das klingt besser, als es möglicherweise ist. Hat nämlich der Bund seine Aufgabenzuweisung aus einem Sozialleistungsgesetz gestrichen und ändert er erst später den Inhalt der Aufgabe mit der Folge einer steigenden Aufgabenlast, so muß das Land dafür nur einstehen, wenn es einen eigenen Zurechnungsakt setzt. Läßt es seinerseits eine schon bestehende Aufgabenübertragung unverändert, so soll es nach verbreiteter Ansicht an einer solchen Zurechnung fehlen. ${ }^{51}$

Aus diesem Dilemma gibt es für die Kommunen rechtlich gesehen ${ }^{52}$ nur zwei Auswege: Entweder es gelingt eine Auslegung des zweistufigen Schutzmechanismus, mit der dessen Löchrigkeit geflickt wird. 53 Oder es werden zumindest im Rahmen des kommunalen Finanzausgleichs die bundesrechtlich verursachten Mehrausgaben in die

49 Ausführlich dazu Becker, Der Schutz der Kommunen vor Aufgabenänderungen - Zu Aufgabenübertragungsverbot und Konnexitätsgebot am Beispiel des Bildungs- und Teilhabepakets und des Vormundschaftsänderungsgesetzes, Gutachten im Auftrag der kommunalen Spitzenverbände NRW, Materialien des Deutschen Städtetags 2012.

50 Erste Regelung in Art. 71 Abs. 3 LV BW v. 11.11.1953; letzte Regelung Art. 57 Abs. 4 Nds. Verf., eingeführt durch Gesetz zur Änderung der Nds. Verf. und des Gesetzes über den Staatsgerichtshof v. 27.1.2006 (Nds. GVBl., S. 58), rückwirkend in Kraft getreten zum 1.1.2006. Eine chronologische Darstellung der Einführung von Konnexitätsregelungen in den Landesverfassungen findet sich bei Henneke, Die Kommunen in der Finanzverfassung des Bundes und der Länder, 4. Aufl. 2008, S. $139 \mathrm{ff}$.

51 Engelken, Wenn der Bund seine alten Aufgabenzuweisungen an Kommunen aufhebt, DÖV 2011, S. 745, 750 f.; Engelbrecht, „Große Erwartungen“ - das „Kinderkrippen“-Urteil des VerfGH NRW vom 12.10.2010 und seine Auswirkungen für die bayerischen Kommunen, BayVBl. 2011, S. 718, $720 \mathrm{f}$.

52 Die dritte Lösung besteht in einer politischen Verhandlung über eine Kostenübernahme durch den Bund, vgl. etwa zum Beispiel der Finanzierung der Eingliederungshilfe durch den Bund als ,,politische Gegenleistung“ für die Zustimmung der Länder im Bundesrat zum Europäischen Fiskalpakt http://www.zeit.de/politik/deutschland/2012-06/fiskalpakt-bund-laender, abgerufen am 24.7.2012.

53 Vorschlag bei Becker, Der Schutz der Kommunen vor Aufgabenänderungen (Fußn. 49), S. 61 ff. 
Rechnung eingestellt, wie es der VerfGH Rhld.-Pfalz in einer jüngst ergangenen Entscheidung gefordert hat ${ }^{54}$ - wobei allerdings eine genaue Gegenrechnung nicht erfolgt.

IV. Schluß

Eine Sozialraumperspektive eröffnet - gerade im Zusammenhang mit einer umfassenden Querschnittsaufgabe wie der Inklusion von Menschen mit Behinderungen - einen Blick auf das vielfältige und oft schwierige Zusammenspiel unterschiedlichster hoheitlicher Maßnahmen.

Auch wenn räumliche Bezüge verschieden gegliedert sein können, unterstreicht diese Perspektive zugleich die wieder zunehmende Bedeutung einer kommunalen Aufgabenwahrnehmung, sowohl bezogen auf die Gewährung von Sozialleistungen als auch die Bereitstellung von Dienstleistungen und Einrichtungen der allgemeineren Daseinsvorsorge.

Diese Aufgabenwahrnehmung bleibt im Rahmen der deutschen verfassungsrechtlichen Ordnung komplex. Sie erfordert vor dem Hintergrund der Trennung von Bundesund Landesstaatsgewalt und der dadurch erschwerten Finanzbeziehungen die Abstimmung zwischen Bund, Ländern und Kommunen, zwischen verschiedenen kommunalen Ebenen, zwischen Kommunen und anderen Behörden sowie zwischen kommunalen Trägern und dritten Leistungserbringern.

Daraus resultieren vielfältige Herausforderungen bei der Erreichung des Ziels, allen Menschen auch in einem materiellen Sinn gleiche Teilhabechancen zu gewährleisten. Diese konnten und sollten hier nur angedeutet werden. Ihre nähere Herausarbeitung aus verschiedenen Blickwinkeln ist Gegenstand der in diesem Band im Folgenden abgedruckten Beiträge. 


\title{
Überall und nirgendwo - \\ „Disability Mainstreaming“ im kommunalen Lebensraum und Sozialraumorientierung als Transformationskonzept
}

\author{
Elisabeth Wacker
}

I. Nirgendwo - die (Un-)Sichtbarkeit von Behinderung und der Lebenschancenansatz

II. Sozialraum und ICF - Ansatzpunkte zur Transformation eines Lebensraums für alle

III. Überall - „Mainstreaming Disability“ als Entwicklungsprogramm und -methode

IV. Ausblick - evidenzbasierte Maßstäbe für Leistungssysteme als Königsweg?

V. Literatur

Die Veränderung kommunaler Praktiken im Umgang mit Personen mit Beeinträchtigung und Benachteiligung lohnt grundlegend neuer sozialwissenschaftlicher Aufmerksamkeit, auch wenn oder gerade weil geradlinige Prozesse des Wandels schwer aufzuzeigen sind. Vielmehr liegen - wie der Wissenschaftshistoriker Rheinberger ${ }^{1}$ dies beschreibt - wie in einem Bergwerk Goldadern nicht offen, sondern müssen entdeckt werden. Zwar stehen Wege in Form bereits gegrabener Tunnel und Schächte den Suchenden zur Verfügung, diese sind aber teilweise verwirrend angeordnet und Sicherheit, zielführend unterwegs zu sein, ist nicht einfach zu erlangen. Deswegen mag ein multidisziplinärer Blick zunächst als Verwirrung erscheinen, aber er kann auch als VerheiBung gedeutet werden, der Gefahr eindimensionaler Pfadfindung aus spezifisch fachlicher Perspektive zu entrinnen.

Wenn die Frage lautet, wie im Sozialraum Neues entsteht nach der Maßgabe einer aktuellen Vision der inklusiven Gesellschaft, so kann es verschiedene fachliche Tunnel und methodische Schächte geben, um dem Kern des Wissens näher zu kommen, das im Licht der Behindertenrechtskonvention der Vereinten Nationen (UN-BRK) ${ }^{2}$ neu zu betrachten ist. Der hier abgesteckte „Claim“ bezieht sich auf einen noch utopischen Le-

1 Rheinberger, Man weiß nicht genau, was man nicht weiß. Über die Kunst, das Unbekannte zu erforschen, NZZ vom 5.5.2007.

2 Das Übereinkommen der Vereinten Nationen vom 13. Dezember 2006 über die Rechte von Menschen mit Behinderung sowie das Fakultativprotokoll zum Übereinkommen sind in Deutschland seit 2009 geltendes Recht in Form einfachen Bundesrechts (BGBl. II 2008, S. 1419 ff.). 
bensraum „Kommune“, in dem Merkmale und Möglichkeiten von Transformationskonzepten in drei Gedankensträngen aufgezeigt werden sollen: erstens die Sichtbarkeit von Behinderung durch einen Lebenschancenansatz, zweitens der Wandel von Sozialräumen zum Lebensraum aller und drittens die Potenziale eines „Disability Mainstreaming“ als Katalysator kommunaler Entwicklung3.

\section{Nirgendwo - die (Un-)Sichtbarkeit von Behinderung und der Lebenschancenansatz}

Es gibt einige Bevölkerungsgruppen wie „die Alten“, „die Arbeitslosen“ oder „,die Alleinerziehenden“, deren gesellschaftliche Verankerung fragil erscheint. Aber „die Behinderten“ befinden sich in Gefahr, traditionell und von Anfang an zu den „Ausgeschlossenen“" zu zählen.

Das Risiko einer Kombination von Ungleichheit und Benachteiligung tritt bei physisch, psychisch oder sinnesbeeinträchtigten Menschen charakteristisch auf. Dies kann mehrere Gründe haben:

- es ist deutsche Tradition, dass Maßnahmen und Aktivitäten von Politik und Leistungsträgern dann einsetzen, wenn Behinderung eingetreten ist;

- noch immer wird Behinderung vorrangig so betrachtet, wie es Prüfdaten medizinisch-therapeutischer Diagnostiken oder leistungsbezogene Verwaltungsstatistiken nahelegen (z.B. schädigungsorientiert bzw. nach Grad der Behinderung: GdB);

- Leistungen erfolgen vorrangig über den Einsatz „besonderer Maßnahmen“ (bzw. in Sondereinrichtungen).

Daher wird die Exklusion dieses Personenkreises über Strukturen und Organisationen verfestigt ${ }^{4}$, die wiederum die Inklusionschancen am Arbeitsmarkt, in privaten und öffentlichen Organisationen, in allen Stufen des Bildungssystems (von der Vorschule bis zur Universität), bei politischer Willensbildung und -gestaltung, aber auch im Bürgerschaftlichen Engagement sowie in der alltäglichen Lebensführung beeinflussen. Dies führt in einen „Teufelskreis“.

Die tatsächlichen Lebensbedingungen der behinderten Menschen sind hingegen kaum im Blickfeld, hier fehlt es also

- an gesellschaftlicher Wahrnehmung und Problembewusstsein,

- an wissenschaftlich fundierten Kenntnissen zur Lebenslage von Menschen mit Beeinträchtigungen, um besser zu wissen und zu verstehen, inwieweit räumliche,

3 Grüber, Disability Mainstreaming, IMEW konkret Nr. 10, Dezember 2007, abrufbar unter: www. imew.de/index.php?id=237 (letzter Zugriff: 19.03.2011).

4 Bude/Lantermann, Soziale Exklusion und Exklusionsempfinden, Kölner Zeitschrift für Soziologie und Sozialpsychologie 58/2006, S. 233-252. 
soziale und Bedingungen der Infrastruktur gleichberechtigte Teilhabe bei Beeinträchtigung behindern sowie

- an Forschung zu Potenzialen sowie ihrem Aufbau bzw. ihrer Stärkung, damit es den sehr verschieden beeinträchtigten Menschen gleichermaßen möglich wird, selbstbestimmt und ohne Teilhabeeinschränkungen zu leben.

Eine Ungleichheit scheint allerdings in rechtlicher Perspektive zunächst kaum kenntlich, denn die Maßgabe: „Niemand darf wegen seiner Behinderung benachteiligt werden" ist seit nunmehr beinahe zwei Jahrzehnten in Artikel 3 des Grundgesetzes $\left(\mathrm{GG}^{5}\right)$ aufgenommen. Dieses Diskriminierungsverbot enthält allerdings paradoxe Züge, folgt man einer sozialwissenschaftlichen Definition von Behinderung, die genau in der Benachteiligung der Beeinträchtigten ihren Ausgangspunkt nimmt (d.h. Behinderung bedeutet eben keine Wesenheit oder Eigenschaft einer Person, sondern die Benachteiligung der Personen mit dem Merkmal einer oder mehrerer Beeinträchtigungen). Hier könnte mit der Aufnahme eines Verbots der „Benachteiligung von Behinderten“ ins Grundgesetz sachlogisch also ein Pyrrhussieg errungen sein, gerade im Sinne der Gleichstellung beeinträchtigter Menschen. Denn die Abweichung durch Beeinträchtigung (des Sehens, Gehens, Entscheidens, Orientierens oder anderer Funktionalitäten) wird scheinbar unverrückbar mit Behinderung gekoppelt.

Gesellschaftliche Realität wird in einer aktuellen Umfrage der Aktion Mensch erkennbar, die belegt, dass über die Hälfte der Bevölkerung in Deutschland behinderten Menschen im Alltag nicht begegnet. Zwar wünschen sich demnach $87 \%$ der Deutschen ein Zusammenleben auf gleicher Augenhöhe, aber jede/r Dritte gibt an, gar keinen Kontakt zu Menschen mit Beeinträchtigung zu haben 6 . Die Exklusion scheint also Normalität zu sein, obwohl seit dem Jahr 2001 ein eigenes Sozialgesetzbuch (SGB IX ${ }^{7}$ ) Rehabilitation und Teilhabe als sozialpolitische Zielorientierung ausweist.

Die mit der UN-BRK (s.u. III.) von Deutschland unterzeichnete Zielsetzung gesellschaftlicher Inklusion bei Beeinträchtigung könnte daher als aus menschenrechtlicher Perspektive getriebene Vision interpretiert werden, auf die es vor allem hinzuarbeiten gilt. Die Existenz von Behinderung alltagsnah in einer inklusiven Gesellschaft aufzuheben, wäre dann schrittweise zu entwickeln, indem

- nach einem Lebenschancenansatz Beeinträchtigung und Benachteiligung in ihrem Zusammenwirken identifiziert und sichtbar werden und zugleich

- präventive, rehabilitative und soziale Leistungen bezogen auf ihre Wirkung beim Ausgleich von Benachteiligung geprüft werden, nach dem Maßstab der Teilhabegerechtigkeit.

5 Grundgesetz für die Bundesrepublik Deutschland (1949/Stand: 2012) BGBl. I 1994, S. 3146.

6 www.aktion-mensch.de, zit. nach Rehabilitation 2012; S. 51, 132.

7 Sozialgesetzbuch Neuntes Buch - Rehabilitation und Teilhabe behinderter Menschen, BGBl. I 2001, S. 1046. 
Der Lebenschancenansatz überschreitet traditionelle Zuordnungen zu sozialen Bezugskonzepten, wie Klasse, Schicht oder Milieu, zugunsten einer Diversitätsperspektive mit Aufmerksamkeit für verschiedene Lebenslagen und Ungleichheitsmerkmale in ihnen, wie Alter, Bildung, Ethnie, Funktionalität/Gesundheit/Körper, Geschlecht, Nationalität, Sexualität, Religion etc. ${ }^{8}$. In einem prozessorientiert angelegten Zugang kann reflektiert werden, ob es sich lohnt, Charakteristika des Lebens mit Behinderung in der Gesellschaft (bzw. die Verfügbarkeit von Ressourcen bei Beeinträchtigungen) entlang verschiedener Merkmale (Differenzlinien) zu verdeutlichen und zugleich nach deren verstärkenden, aufhebenden oder mildernden Wirkzusammenhängen zu fragen, wie dies beispielsweise die Intersektionalitätsforschung fordert und entwickelt ${ }^{9}$. Dieser mehrdimensionale Zugang kann zugleich den Druck mildern, sich bei einer vorliegenden Benachteiligung zugunsten eines Leistungsbezugs einer bestimmten Gruppe zuzuordnen bzw. ihrer Interessenvertretung zu unterwerfen.

Das Allgemeine Gleichbehandlungsgesetz $\left(\mathrm{AGG}^{10}\right.$ ) aus dem Jahre 2006 soll Benachteiligung verhindern oder beseitigen beim Zugang zum Arbeitsleben, zur Gesundheitsversorgung, Bildung oder anderen Gemeinschaftsgütern. Es nennt mehrere Merkmale, anhand derer Ungleichbehandlung und Benachteiligung sichtbar werden kann: In $\S 1$ „Ziel des Gesetzes“ werden Rasse oder ethnische Herkunft, Geschlecht, Religion oder Weltanschauung, Behinderung, Alter oder sexuelle Identität aufgezählt als mögliche Auslöser von Benachteiligung. Unterschieden wird weiter zwischen

- unmittelbarer Benachteiligung §3 Abs. 1 im Fall einer weniger günstigen Behandlung einer Person in vergleichbaren Situationen,

- mittelbarer Benachteiligung $\S 3$ Abs. 2 im Fall scheinbar neutraler Vorschriften, die Personen mit den genannten Merkmalen benachteiligen,

- Belästigung bzw. sexueller Belästigung § 3 Abs. 3 und 4 als Benachteiligung, die die Würde einer Person und ihre sexuelle Integrität verletzt und der

- Anweisung zur Benachteiligung $\S 3$ Abs. 5.

8 Sen, Ökonomie für den Menschen, München 2007; Voges/Jürgens/Mauer/Meyer, Methoden und Grundlagen des Lebenslagenansatzes, ZeS: Zentrum für Sozialpolitik Endbericht, 2003, www. soziologie.uni-kiel.de/bergersozun/Voges_Lebenslagenansatz.pdf.

9 Vgl. Baer/Bittner/Göttschke, Mehrdimensionale Diskriminierung - Begriffe, Theorien und juristische Analyse. Teilexpertise im Auftrag der Antidiskrimimierungsstelle des Bundes, Berlin 2011; Dern/Inowlocki/Oberlies, Mehrdimensionale Diskriminierung - Eine empirische Untersuchung anhand von autobiographisch-narrativen Interviews. Teilexpertise im Auftrag der Antidiskriminierungsstelle des Bundes, Berlin 2011; Lutz/Wenning, Differenzen über Differenz - Einführung in die Debatten, in: dies. (Hrsg.), Unterschiedlich verschieden. Differenz in der Erziehungswissenschaft, Opladen 2001, S. 11-24; Minow, Making All the Difference. Inclusion, Exclusion and American Law, Ithaka, New York 1990; Young, Justice and the Politics of Difference, Princeton, New Jersey 1990; Zinsmeister, Mehrdimensionale Diskriminierung. Das Recht behinderter Frauen auf Gleichberechtigung und seine Gewährleistung durch Art. 3 GG und das einfache Recht, Baden-Baden 2007.

10 Allgemeines Gleichbehandlungsgesetz, BGBl. I 2006, S. 1897. 
Auf der Basis des AGG wird es möglich, auch mehrere Diskriminierungsgründe gemeinsam zu berücksichtigen ( $\$ 4$ : „Unterschiedliche Behandlung wegen mehrerer Gründe").

Aus der Perspektive eines Lebenschancenansatzes lässt sich Behinderung in der Gesellschaft folglich sichtbar machen ${ }^{11}$, indem

- die generelle Rolle und Position beeinträchtigter Menschen in der jeweiligen Gesellschaft thematisiert wird,

- Differenzen deutlich werden zur allgemeinen Lebenslage und

- soziale Leistungen für diesen Personenkreis und ihre Wirkungen und Nebenwirkungen auf den Prüfstand gestellt werden können.

Merkmale sozialer Beteiligung bzw. Benachteiligung im Arbeitsleben, am Wohnungsmarkt, bei der Mobilität, der Information und Kommunikation etc. werden benennbar.

Entsprechende Fragen können systematisch der Struktur gesellschaftlicher Binnendifferenzierung folgen und prüfen, wie der Zugang zu Gütern wie

- Waren und Dienstleistungen,

- politischem System,

- Bildungssystem,

- kulturellem Leben und

- Gesundheitssystem,

um nur einige Beispiele zu nennen, konkret behindert bzw. möglich wird.

Wirkungen von Maßnahmen können als Reduktion von Exklusion im Sinne unfreiwilligen Ausschlusses evident und ihre Auslöser damit erkennbar werden.

Dieser Zugang ist national ebenso wie transnational fruchtbar und kann bei systematischer Umsetzung ein Monitoring jeweiliger nationaler und binnenstruktureller (kommunaler) Entwicklungen ebenso ermöglichen wie die internationalen Kooperationen und Vergleiche, die die UN-BRK einfordert (Art. $31 \mathrm{ff}$.).

Über Bezugsgrößen des Lebenschancenansatzes werden neben Aussagen zur Lebenslage auch Ausprägungen von Inklusion und Exklusion identifizierbar, die soziale Anerkennung (Toleranz, Akzeptanz, Respekt ${ }^{12}$ ) und Zugehörigkeit verschieden beeinträchtigter Menschen in einer Gesellschaft über Beobachtung und Befragung (Selbstund Fremdauskünfte) abbilden.

Ziel dieser Strategie ist, Behinderung in der Gesellschaft sichtbar zu machen und so den Weg zur sozialen Inklusion zu ebnen, der vom Rat der Europäischen Union als Prozess beschrieben wird:

11 Wacker, Behinderung in der Gesellschaft. 50 Jahre im soziologischen Blick - vom Dialog zum Diskurs, Geistige Behinderung 47/2008, S. 42-61.

12 Sennett, Respekt im Zeitalter der Ungleichheit, Berlin 2004. 
„Social inclusion is a process which ensures that those at risk of poverty and social exclusion gain the opportunities and resources necessary to participate fully in economic, social and cultural life and to enjoy a standard of living and well-being that is considered normal in the society in which they live. It ensures that they have greater participation in decision making which affects their lives and access to their fundamental rights." 13

Auf welche Weise sich diese definierten Rechte auf Chancen und Ressourcen zur vollen Teilhabe und auf angemessene Standards von Lebensqualität und Wohlergehen realisieren, kann nicht alleine dem sozialpolitischen Spiel der Kräfte überlassen bleiben, sondern verpflichtet ebenso die Wissenschaft (insbesondere auch eine an Grundlagen orientierte Wissenschaft), die Frage zum Gegenstand zu machen.

Wie kann diese im Lebenschancenansatz operationalisierbare Aufgabe nun konkret werden?

\section{Sozialraum und ICF - Ansatzpunkte zur Transformation eines Lebensraums für alle}

Seit Jahren befassen sich insbesondere Sozialarbeit und Sozialpädagogik in ihren Analysen und Erprobungen mit dem Sozialraum ${ }^{14}$. An ihre Arbeiten kann angeknüpft werden. Sie betrachten den Sozialraum nicht einfach als einen Ort, sondern als Geschehensfeld, in dem das von Menschen konstruierte und immer aufs Neue rekonstruierte Leben Tag für Tag erkennbar wird. Dieser Alltagsraum ist angereichert mit Gefühlen, Möglichkeiten und Hindernissen. Hier realisiert sich regelgesteuertes und lebensweltliches Zusammenleben von Menschen. Lokalisiert im Dazwischen von Gesellschaft und Individuum ${ }^{15}$ richtet sich im Sozialraum Aufmerksamkeit auf die Wechselwirkungen zwischen sozialen Konstruktionen und Handelnden sowie auf deren Verflechtung. Insofern müssen auch Fragen, die sich auf soziale Wirklichkeit beziehen, auf Menschen in Situationen der Behinderung und Behinderungserfahrungen, im Kontext des Sozialrau-

13 Council of the European Union, Joint report by the Commission and the Council of social inclusion, SOC 115 ECOFIN 80 EDUC 46 SAN 49, Brüssel 2004, S. 10.

14 Bingel, Sozialraumorientierung revisited. Geschichte, Funktion und Theorie sozialraumbezogener Sozialer Arbeit, Wiesbaden 2011; Früchtel/Budde/Cyprian, Sozialer Raum und Soziale Arbeit. Textbook: Theoretische Grundlagen, Wiesbaden 2007; Hinte, Eigensinn und Lebensraum - zum Stand der Diskussion um das Fachkonzept „Sozialraumorientierung“, VHN 78/2009, 20-33; Kessl/Reutlinger, Sozialraum, in: Otto/Thiersch (Hrsg.), Handbuch Soziale Arbeit, 4. Aufl. München \& Basel 2011, S. 1508-1516.

15 Bertschi, Im Dazwischen von Individuum und Gesellschaft. Topologie eines blinden Flecks der Soziologie, Bielefeld 2010. 
mes aufscheinen. Weisser ${ }^{16}$ pointiert diese Beobachtungsgegenstände als sozialräumlich materialisierte Interessenkonflikte, d.h. als Situationen, in denen das Vermögen, etwas zu realisieren, ungleich verteilt ist, weil nicht alle Fähigkeiten gleich gefragt sind, nicht alle Chancen gleich eröffnet bzw. nicht alle Ressourcen gleich vorhanden, zugänglich und nutzbar sind. Diese Konflikte zwischen dem, was (für jemanden) gerade möglich, und dem, was gerade gefordert ist, werden beobachtbar im Feld der Verhältnisse und des Verhaltens.

Schon in den 1980er Jahren wurde - bewusst in Spannung zu damaligen integrationspädagogischen Forderungen nach Dezentralisierung und Regionalisierung der Hilfen - eine Debatte geführt um „Orte zum Leben“ für gesellschaftlich Ausgeschlossene. Der deutsche Protagonist Gaedt ${ }^{17}$ schrieb der Behindertenhilfe ins Pflichtenheft, solche Orte zu schaffen für die häufig als strukturelle Verlierer einer mehr und mehr binnendifferenzierten und individualisierten Gesellschaft erscheinenden behinderten Menschen. Nicht in den Gemeinden sollten sie freigesetzt und damit ausgesetzt leben (müssen), sondern in speziell für sie zugeschnittenen Heimaten der traditionellen Leistungsanbieter, wie Diakonie oder Caritas. Aus der Perspektive der Anbieter dieser professionell geführten Schon- oder Förderräume sollte dort ein unverantwortlicher Entzug von Ressourcen für Schutzbedürftige verhindert werden, wogegen inklusives Planen und Denken Personen, die gerade durch ihre soziale Abhängigkeit charakterisiert seien, dem freien Spiel der Kräfte aussetze und so ihre Eigenentwicklung und ihre gesellschaftliche Wertschätzung gefährde (d.h. sie unangemessen gleich behandele).

Repräsentative Studien zeigten allerdings bereits zeitgleich, dass sich die zu Orten der Einbindung und Einbettung stilisierten Komplexeinrichtungen, in denen sich Einschränkungen und Abweichungen von gesellschaftlichen Leistungs- und Verhaltenserwartungen auf behinderte Menschen nicht negativ auswirken sollten, gerade als Organisationen mit hohem Regulierungs- und Reglementierungspotenzial gegenüber den dort Platzierten und Tätigen entpuppten, also ihre selbstbestimmte Teilhabe limitieren ${ }^{18}$.

$\mathrm{Ob}$ - jenseits solcher seit Jahrzehnten etablierten Konfliktlinien - mit Hilfe des Fachkonzepts der Sozialraumentwicklung nach objektiven Kriterien für verwirklichte Teilhabechancen gesucht werden kann, verdient daher wissenschaftlicher Aufmerksamkeit. Denn das Sozialraumkonzept will Divergenz und Differenz ebenso einbeziehen wie auf neue Steuerungsmechanismen für ein Leben in der Gemeinschaft aller achten ${ }^{19}$. Damit wäre es mehr als ein reines „Zuteilungsverfahren“ mit dem eingeschränkten

16 Weisser, Sozialraumorientierung und Situationen der Behinderung - Über die sozialräumliche Strukturierung von Abhängigkeitsbeziehungen, VHN 79/2010, S. 4, 6.

17 Gaedt, Einrichtungen für Ausgeschlossene oder „Ein Ort zum Leben“. Überlegungen zur Betreuung Geistigbehinderter, Jahrbuch für kritische Medizin Bd. 7, Berlin 1981, S. 96-109.

18 Wacker/Wetzler/Metzler/Hornung, Leben im Heim. Angebotsstrukturen und Chancen selbständiger Lebensführung in Wohneinrichtungen der Behindertenhilfe, Schriftenreihe des BMG 102, BadenBaden 1998.

19 So Hinte (Fußn. 14), S. 25. 
Blick auf Fall, stationäre oder ambulante Immobilie oder leistungssystematische $\mathrm{Zu}$ ständigkeit im territorialen Bezug. ${ }^{20}$

Kompliziert wird die erforderliche Kriteriensuche allerdings, weil gewissermaßen neue Erkenntniswege zu graben sind, wenn nicht nur eine bestehende kommunale Wirklichkeit abgebildet und als Bezugspunkt genutzt werden soll, sondern es darum geht, die zukünftigen Chancen und Grenzen der gesellschaftlichen Teilhabe bei Behinderung zu bemessen. Derzeit verstellen hierfür traditionelle Versorgungsmechanismen eher den Blick, und gute Beispiele der Teilhabe, die Schule machen und Transformationen bewirken könnten, sind rar.

Wie bei Emanzipationsprozessen nicht unüblich, muss also das Zusammenspiel von individueller Agency (Handlungspotenzial ${ }^{21}$ ) und sozialen Strukturen (d.h. auch der Sozialpolitik und des Sozialrechts) erfolgen unter Bedingungen der Ungleichzeitigkeit, insofern Akteure und Akteurinnen einbezogen sind, die derzeit in ihrem Zugang zu Ressourcen behindert sind und deren Verschiedenheit benachteiligt wird. Denn derzeit sind Menschen mit Beeinträchtigung eher gehalten, ihre Verschiedenheit zu verleugnen oder zu reduzieren (d.h. so „normal“ wie möglich zu erscheinen), um uneingeschränkt im sozialen und kommunalen Feld (und dort in Systemen wie Bildung, Mobilität, Politik etc.) teilhaben zu können.

Transformation ist also erforderlich, d.h. ein wesentlicher Wandel zu einer inklusiven Gesellschaft. Sie soll sich vollziehen durch die

- Institutionalisierung und Konsolidierung neuer, auf Inklusion gerichteter Leistungssysteme,

- Veränderung eines bestehenden defizitorientierten Bildes über beeinträchtigte Menschen und Selbstbildes beeinträchtigter Menschen,

- Entwicklung neuer Handlungs- und Deutungsmuster,

- Umformung von Strukturen und Architekturen und

- Umsetzung völkerrechtlicher Verträge in nationales Recht (insbesondere der UNBRK).

Ein erster Schritt wird sein, jenseits des Warenkorbs des bestehenden Nationalen Aktionsplans und vieler Landesaktionspläne, nicht nur eine klare Zielorientierung auf Inklusion anzubahnen, sondern dies auch mit einer Neudeutung bestehender Denkstrukturen über Behinderung zu verbinden. Der schrittweise Umbau vieler konzeptioneller, struktureller und lokaler Elemente muss gelingen, um den Übergang aus exkludierenden Kulturen und den neuen Zugang zu förderlichen Ressourcen im Alltag für beeinträchtigte Menschen zu eröffnen, d.h. zu Macht, Geld und Sinn.

20 Siehe die Debatte um ein „Sozialraumbudget“ zur Bearbeitung sämtlicher in einem Gebiet anfallenden Leistungsansprüche (nach $\S \S 27$ ff. SGB VIII), damit ein effektiverer und effizienterer Mitteleinsatz gewährleistet wird und auch präventive und auf Umgebungen (Netze) bezogene Entwicklungen finanziert werden können (Hinte (Fußn. 14), S. 26).

21 Bandura, Social cognitive theory: An agentic perspective, Annual Review of Psychology 52/2001, S. 1-26. 
Abb. 1 Ein Beispiel für Transformation: EsCHER „Luft und Wasser“ 22

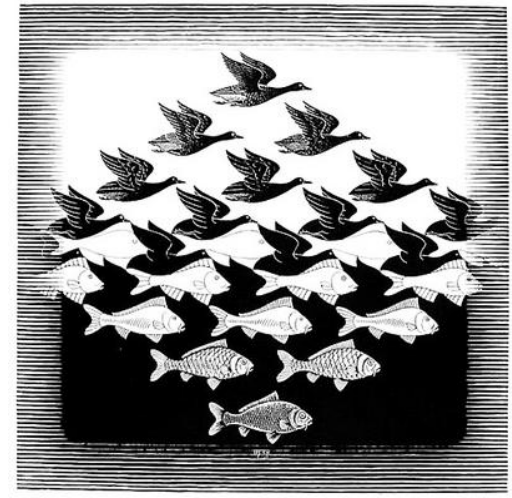

Inwiefern sich diese Transformationsprozesse durch Sozialraumorientierung vollziehen können, ist bislang kaum erforscht oder erprobt. Die Stärke einer Orientierung am sozialen Raum könnte im Blick auf Verbindungen und Interdependenzen liegen, die zugleich im transdisziplinären Diskurs (z.B. u.a. von Betriebswirtschaft, Gesundheitswissenschaft, Raumplanung, Sozialrecht und -politik) reflektiert werden können. Hier würde, bezogen auf den Personenkreis behinderter Menschen, weitgehend Neuland beschritten.

Hilfreich könnte die weltweit institutionalisierte Grammatik für den Verständigungsprozess darüber sein, Beeinträchtigungen nicht als „Eigenschaft einer Person“, sondern als Teil der menschlichen Verschiedenheit und Vielfalt zu verstehen. Dies ist verfasst in der Internationalen Klassifikation von Funktionsfähigkeit, Behinderung und Gesundheit (der ICF), wie sie von der WHO ${ }^{23}$ seit dem Jahr 2001 vorliegt. Dieses als ,,bio-psychosozial“" bezeichnete Modell betrachtet komplexe (Wechsel-)Wirkungszusammenhänge der körperlichen, individuellen und gesellschaftlichen Dimensionen einer Behinderung und kann auch als international einsetzbare Richtschnur für die notwendigen Monitoringverfahren im Gesundheitssystem und der Rehabilitation dienlich sein.

Mit den Domänen der ,International Classification of Functioning, Disability and Health" 24 wird ein neuer multiperspektivischer Behinderungsbegriff operationalisierbar, der die sozialrechtlich üblichen Definitionen überschreitet. Es liegt daher nahe, sie als Transformator auf dem Weg zur geplanten und gestalteten neuen Community zu nutzen, weil sie nicht nur Behinderung sichtbar werden lässt, sondern auch einem über räumliche Perspektiven hinausreichenden Sozialraumbegriff genügt. Wesentlich ist der multiperspektivische Zuschnitt, der auch komplexe Lagen erfassen und aufdecken kann:

22 http://www.kunstkopie.de/a/escher-mc/luftundwasser8.html.

23 WHO - World Health Organization, International Classification of Functioning, Disability, and Health (ICF), Geneva 2001. Dt.: DIMDI - Deutsches Institut für Medizinische Dokumentation und Information (Hrsg.), Internationale Klassifikation der Funktionsfähigkeit, Behinderung und Gesundheit, Genf 2005, http://www.dimdi.de/static/de/klassi/icf/index.htm (Zugegriffen: 02. Januar 2013).

24 WHO (Fußn. 23). 
- Neben einer körperbezogenen Perspektive (body perspective) bietet sie

- eine personenbezogene Perspektive (individual perspective) sowie

- eine gesellschaftsbezogene Perspektive (societal perspective).

Insofern werden jeweils Körperfunktionen und -strukturen bzw. deren Beeinträchtigung (body functions and structures), Handlungsräume bzw. deren Einschränkungen (domains of activity, d.h. etwas tun können - capacity - und tun - performance) und Teilhabechancen bzw. deren Begrenzungen (domains of participation) betrachtet, was bereits die Aufmerksamkeit für deren komplexe und dynamische Interaktionen und Interrelationen schärft.

Abb. 2 International Classification of Functioning, Disability and Health ${ }^{25}$

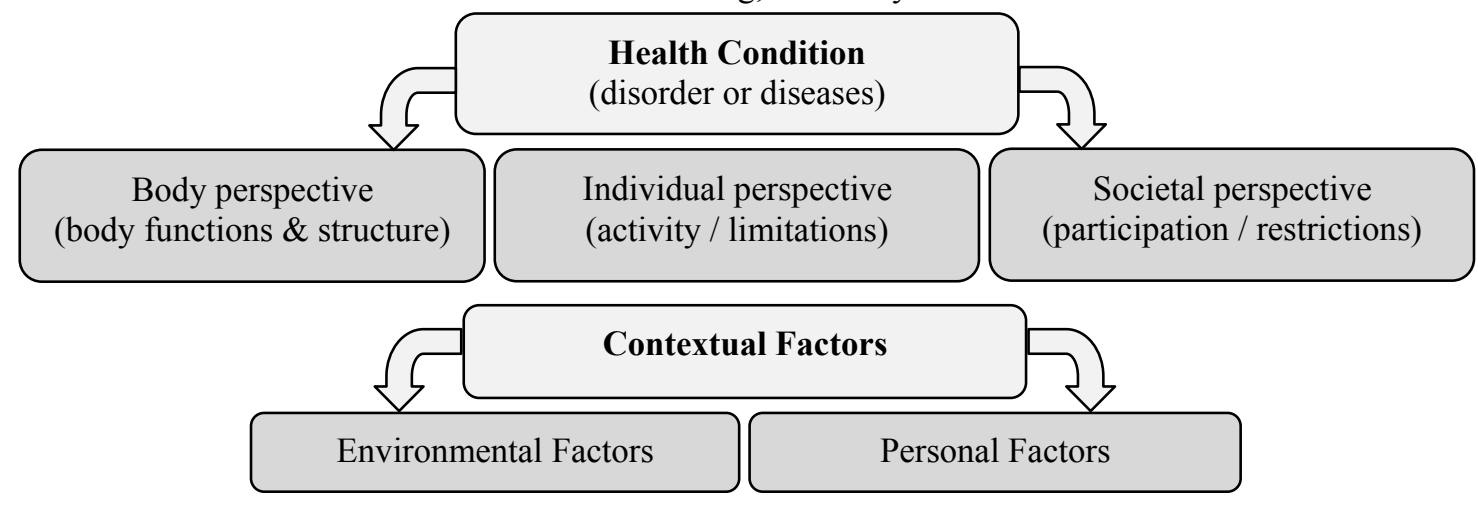

Schließlich geht es um die sog. Kontextfaktoren, die die möglichen Wechselbeziehungen zwischen Funktionalität, Aktivität und Partizipation in der Perspektive der jeweiligen Person und ihrer Umwelt abbilden sollen und damit aufzeigen, dass sie kontextsensibel, relativ und relational sind. Der Lebenshintergrund einer Person, der förderlich oder hinderlich wirken kann, gewinnt somit erhebliche Bedeutung, über eine reine Betrachtung von (chronischen) Krankheiten oder Funktionsstörungen hinaus.

Bei den Umweltfaktoren (environmental factors) ist die materielle, soziale und einstellungsbezogene Umwelt gemeint, in der Menschen leben und die ihr Leben mitgestaltet. Umweltbezogene Elemente sind konkret

- die dingliche Umwelt: Produkte und Technologien (z.B. Hilfsmittel, Barrieren im Lebensumfeld, Medikamente),

- die natürliche und vom Menschen veränderte Umwelt (in Form von Räumen, Gebäuden, Verkehrswegen),

- die soziale Umwelt: Angehörige, Partnerinnen bzw. Partner und Familienkreis (bis zu Haustieren), Freunde und Bekannte, Unterstützungs- und Pflegepersonen, medizinische, therapeutische, pädagogische, psychologische und andere Fachleute und die Beziehung zu ihnen wie Assistenz, Begegnung, Beratung, Diagnose, Fürsorge, „Gate keeping“, Therapie etc. 
- die kulturelle Umwelt: also die weitgehend verschlungenen Einstellungen, Urteile, Bewertungen/Beurteilungen, Rollen, gesellschaftlichen Normen, Konventionen und Weltanschauungen sowie schließlich

- die Leistungssysteme: soziale Sicherungssysteme und -dienste und deren Konzepte, das Gesundheitswesen, Gesundheits- und Sozialpolitik, Selbsthilfesysteme, das Bildungs-, Arbeits- und Beschäftigungswesen und seine Träger.

Die personenbezogenen Faktoren (personal factors) hingegen nehmen Bezug auf Gegebenheiten der Person, ihr spezifisches Leben, ihre Erfahrungen sowie individuellen Strategien der Lebensführung und Aufgabenbewältigung. Im Einzelnen werden Aspekte beachtet wie

- Alter,

- Geschlecht,

- ethnische Zugehörigkeit bzw. Zuwanderungserfahrung, aber auch Ressourcen wie

- Erziehung,

- Bildung bzw. Ausbildung,

- Beruf und

- sozialer Hintergrund bzw. Herkunft.

Weitere Charakteristika und Verhaltensmuster, wie Lebensstile und Gewohnheiten, können Risikofaktoren oder Ressourcen sein; vergangene und gegenwärtige Erfahrungen, soziale Kompetenzen und Fähigkeiten (wie Adaptation, „Coping“, Empathie, Flexibilität, Motivation, Vitalität), physische Fitness und psychisches Leistungsvermögen $^{26}$.

Die ICF ist also denkbar als der maßgebliche internationale und fachübergreifende Standard, um Gesundheit und Beeinträchtigung bzw. die Behinderung einer Person (individuelle Ebene) oder in einer Bevölkerung (gesellschaftliche Ebene) zu erfassen und zu beschreiben. Sie beleuchtet im Alltagsbezug die fließenden Grenzen zwischen Gesundheit und Beeinträchtigung (salutogenetischer Ansatz ${ }^{27}$ ) und verdeutlicht die Vielfalt und Verschiedenheit der Beeinträchtigungserfahrungen als Element des Menschseins. Ausgangspunkt ist nicht mehr der Fähigkeitsmangel einer einzelnen Person, sondern sind die Wirkungen verschiedener Umstände der Fähigkeitsentfaltung in konkreten Lebensräumen (from cause to impact). Dadurch treten vor allem die sozialen Aspekte von Behinderung in den Vordergrund, gegenüber einer bio-medizinischen Perspektive von Dysfunktionen. Auch der Blick auf das Leistungssystem wandelt sich. Maßnahmen werden neu bewertet vor der Ausrichtung an einem kommunalen Leistungsgefüge der

26 Schuntermann, Einführung in die ICF: Grundkurs, Übungen, offene Fragen, Hamburg 2005.

27 Antonovsky, Salutogenese: zur Entmystifizierung der Gesundheit (Dt. erw. Hrsg. Franke), Tübingen 1997. 
Rehabilitation (community-based long-term services) ${ }^{28}$. Im Einzelnen geht es um jeweils passende konkrete Wohn- und Lebensverhältnisse, um den Abbau von Barrieren und die Bereitstellung von Hilfsmitteln, um Maßnahmen und Informationen zur Prävention, um geeignete therapeutische Interventionen und Rehabilitation im Lebensumfeld, aber auch um Möglichkeiten der selbstbestimmten Mobilität in der Häuslichkeit und im öffentlichen Raum, um Unterstützungsbedarfe und -bedürfnisse, um verfügbare Assistenz durch Angehörige oder andere relevante Personen (Nachbarn, Freunde, Vereine), um mögliche Selbsthilfepotenziale sowie Bürgerschaftliches Engagement; es geht ebenso um das sozialrechtliche Umfeld (bestehende Leistungsansprüche und den tatsächlichen Zugang zu Leistungen), um wirtschaftliche Rahmenbedingungen, um den Zugang zu und die Nutzung von Information und Kommunikation (Beratung, Medien). Zugleich werden im Bereich Bildung, Ausbildung und Beruf sowohl die Regel- und Sonder/Fördersysteme (Frühförderung, Schulen, Berufsbildungswerke, Berufsförderungswerke, Werkstätten für behinderte Menschen, der Zugang zum Dualen Bildungssystem und zu Hochschulen) als auch die Chancen auf dem allgemeinen Arbeitsmarkt und der Bestand sowie die Wirkung von angepassten Arbeitsplätzen und Integrationsdiensten in den Blick genommen. Dies alles soll ebenso berücksichtigt werden, wie die Vielfalt der Beeinträchtigungsarten und Verschiedenheit ihrer Ausprägung und Bedeutung für konkrete Personen.

\section{III. Überall - „,Mainstreaming Disability“ als Entwicklungsprogramm und-methode}

Der differenzierte Auftrag, im Lebensraum aller (also auch dem kommunalen Raum bzw. dem Quartier) die Lebenschancen der Menschen mit Beeinträchtigung so beschreiben und bewerten zu können, dass Ansatzpunkte für den Prozess einer Entwicklung zur inklusiven Gesellschaft evident werden, wurde reflektiert und operationalisiert. In einem „funktional ausdifferenzierten, demokratischen Sozialstaat[s] wird deutlich, dass Inklusion kein ausschließliches Thema für oder über behinderte Menschen ist, sondern ein rechtlich gesichertes Prinzip der sozialen Einbeziehung der Bevölkerung “29 in Form der Abkehr von rein maßnahmen- und institutionenbezogenem Denken und Handeln ${ }^{30}$. Das Gemeinwesen als „,welcoming community“ soll Zugehörigkeit aller garan-

28 Lightfood, Community-based rehabilitation. A rapidly growing method for supporting people with disabilities, Social Work 47/2004, S. 455-468; Scholz, Die Entwicklung des Community CareAnsatzes in Deutschland und Großbritannien im Vergleich, Norderstedt 2009.

29 Wansing, Der Inklusionsbegriff in der Behindertenrechtskonvention, in: Welke (Hrsg.), UNBehindertenrechtskonvention mit rechtlichen Erläuterungen, Berlin 2012, S. $93,95$.

30 Rat der Europäischen Union, Ziele bei der Bekämpfung der Armut und der sozialen Ausgrenzung (Dokument 14164/1/02 REV 1), Brüssel 2002. 
tieren, auch der behinderten Menschen, aber zukünftig in neuer Form, also nicht mehr als ihre institutionelle Ausgrenzung in der Gesellschaft 31 .

Die Konkretion solcher Überlegungen macht nicht nur Wirkungszusammenhänge sichtbar, sondern auch eine ausgeprägte Komplexität durch die Vielfalt relevanter Items und Denkmodelle. Alleine die menschliche Verschiedenheit ebenso wie die Heterogenität dessen, was als Behinderung gilt, ist erheblich. Es ist daher eine Fiktion, von „den Behinderten“" auszugehen, ohne die Diversität in Alter, Geschlecht, Lebensumständen, Eintritt der Beeinträchtigung, Zugang zu Ressourcen und vieles mehr zu berücksichtigen. Scheinbar im „Überall“" angekommen, liegt nun allerdings die Frage nahe, ob nicht durch die Suche nach Chancengerechtigkeit bei Beeinträchtigung im Sozialraum vor allem eine wissenschaftliche Enttäuschung entdeckt wird: nämlich eine Überkomplexität, die Evidenz kaum möglich macht. Lohnt es dann überhaupt, die eingangs angestrebte Suche fortzusetzen, um mögliche Schätze für ein wissenschaftsbasiertes Entwicklungskonzept zu heben?

Hier soll zu Hartnäckigkeit ermuntert werden. Denn vermutlich gibt es relevante Interdependenzen! Solche Cluster zu identifizieren, wäre ein erster Schritt. Die zu erwartende Komplexität wird wohl die des vor über drei Jahrzehnten ersonnenen magischen Würfels des ungarischen Architekten und Bauingenieurs Rubik nicht unterschreiten, der angeblich einen Anreiz geben wollte, räumliches Denkvermögen zu trainieren. Vielleicht könnte die ICF in Kombination mit Sozialraumentwicklungen zumindest ähnliche Anreize geben, vielleicht geht aber auch mehr!

Denn die Zielorientierung für erforderlichen Wandel ergibt sich aus der Vision der Inklusion, die die UN-BRK als weitere Leitstruktur ins Spiel bringt: als dritte Dimension für die anstehenden Transformationsaufgaben. Die aus der UN-BRK abgeleitete Aufgabe, Behindertenpolitik in allen politischen Gestaltungsfeldern als Querschnittelement zu konkretisieren (sog. Disability Mainstreaming) ${ }^{32}$, eröffnet dann Chancen, dass faktisch bestehende Bedarfe und Bedürfnisse der Menschen, die mit Beeinträchtigungen leben, nicht „,im Übergang“" verlorengehen 33 .

31 Kronauer, „Exklusion“ als Kategorie einer kritischen Gesellschaftsanalyse. Vorschläge für eine anstehende Debatte, in: Bude/Willisch (Hrsg.), Das Problem der Exklusion. Ausgegrenzte, Entbehrliche, Überflüssige, Hamburg 2006, S. 27, 41; ebenso Nassehi, Die paradoxe Einheit von Inklusion und Exklusion. Ein systemtheoretischer Blick auf die „Phänomene“, in: Bude/Willisch (Hrsg.) (Fußn. 31), S. 46, 49 ff.

32 Vgl. Aselmeier, Community Care und Menschen mit geistiger Behinderung, Wiesbaden 2008.

33 Wacker, Behindertenpolitik, Behindertenarbeit, in: Otto/Thiersch (Hrsg.), Handbuch Soziale Arbeit, 4. völlig neu bearbeitete Aufl. München \& Basel 2011, S. 87-100. 
Abb. 3 Lebenschancenansatz

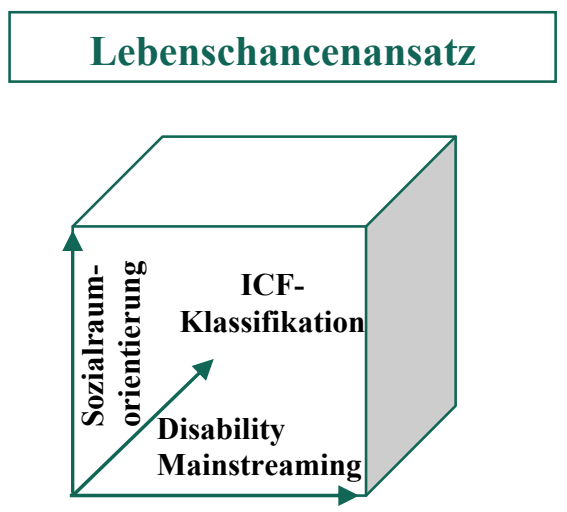

Die UN-BRK artikuliert bezogen auf Behinderung neue Daueraufträge an die Unterzeichner. In der Präambel findet sich die Mission, Behinderungsumstände ständig und dauerhaft als Bestandteil gesellschaftlicher Entwicklungsprozesse zu berücksichtigen: „Emphasizing the importance of mainstreaming disability issues [Hervor. Verf.] as an integral part of relevant strategies of sustainable development" [Buchstabe g] [...].

Zugleich werden Wege aktiver partizipativer Entwicklung und Umsetzung entsprechender politischer Konzepte und Programme festgelegt: „Considering that persons with disabilities should have the opportunity to be actively involved in decisionmaking processes about policies and programmes, including those directly concerning them" [Buchstabe o], [...].

Auch die hiermit erforderliche neue systematische Betrachtung der Zusammenhänge zwischen UN-BRK und Disability Mainstreaming steht noch aus. Hier - wie auch bezogen auf Leistungsansprüche - sind viele Fragen offen und Metamorphosen im Leistungsgeschehen erforderlich. Klar ist aber bereits: Jede genutzte Stellschraube wird zu weiteren Veränderungen der Wirklichkeit von Behinderung in der Gesellschaft führen. Also wird Forschung zu Teilhabe auch einem Geduldspiel gleichkommen, bei dem jede Variation neue Ausgangslagen herbeiführt und man in kleinen Segmenten seinen Forschungsgegenstand jeweils neu beleuchten muss, um neue Passungen zu entdecken.

Grundsatzfragen sind u.a.

- der Umgang mit Vielfalt und Verschiedenheit bei Beeinträchtigung (Diversity Approach und Disability Mainstreaming),

- Möglichkeiten der Selbstbestimmung und Teilhabe,

- die Bedeutung und der Einfluss des demografischen Wandels,

- die Konstruktionen sozialer Sicherung und ihre Möglichkeiten und Grenzen,

- erforderliche neue Dienste und Rollen im gewandelten Unterstützungssystem,

- Relevanz von Subsidiarität und neue (nutzerorientierte) Steuerung der Leistungen,

- Diffusion gewandelter Leitbilder und rechtlicher Rahmensetzungen auf nationaler und internationaler Ebene, 
- Ausprägung von Lebensqualität und Wohlbefinden bei Beeinträchtigung,

- Aufbau generell unterstützender Umwelten (Universal Design bzw. Design für alle) sowie die Entwicklung spezifisch assistiver Technologien (Ambient Assisted Living bzw. selbstbestimmtes Leben durch assistierende Technologien).

Einer wirkungsorientierten Prüfung müssen aber auch Fragen standhalten, wie traditionell institutionell versorgte beeinträchtigte Personen ihren Willen entwickeln, erkennen und einbringen können in einer Weise, die nicht überlagert ist von ihren bisherigen und aktuellen Möglichkeiten und Grenzen der Lebenserfahrung, von gewohnten restriktiven Handlungsspielräumen in „All inclusive-Settings“ und vom Mangel an Alternativen im Unterstützungsgeschehen. Aus anderer Perspektive beleuchtet, geht es um Fragen, wie umfänglich professionelle Kompetenzen, Spezialqualifizierungen und -angebote in sozialräumlichen Ausformungen erforderlich sind und Einfluss nehmen auf eigene Konzepte und Lösungen behinderter Menschen. Im Licht der Subsidiarität ist zu fragen, welche gesellschaftlichen Erwartungen an beeinträchtigte Menschen als engagierte Bürgerinnen und Bürger zu richten wären und wie Grenzen ihrer Eigenleistung oder der Leistungen ihrer sozialen Netze auszuloten sind. Und sieht man aus der Perspektive möglicher Effektivität und Effizienz des Ressourceneinsatzes auf die verschiedenen parallel funktionierenden und agierenden Sozialleistungssysteme, drängt sich die Frage auf, wie nicht oder kaum genutzte systemüberschreitende Kooperationschancen und Pfadleitungen (durch passgenaue Beratung und nutzbare Information und Kommunikation) trägerübergreifend wachsen können in kommunalen Territorien.

Aus dem Blickwinkel gewünschter Lebensqualität und erfahrenen Wohlbefindens sind neben grundsätzlichen Ausstattungen für ein gesundes Leben, die am Zugang zu Grunderfordernissen, wie Sicherheit, Nahrung, Schutz, gesunde Umwelt etc., für alle festzumachen sind, auch neue Konzepte erwägenswert:

- Neue soziale Gemeinschaften, wie sie sich beispielsweise im Internet entwickeln, vernetzen soziale Interessen und haben längst den Status des Nischendaseins verlassen; ,,smart“ organisierte Fahrräder, Autos oder Transaktionen werden Alltag, ebenso könnten sich Gesundheits-, Mobilitäts- und soziale Dienste entwickeln.

- Personenzentrierte Ansätze, wie individuelle Teilhabeplanung, individuelle Leistungsansprüche und -gestaltung im Leistungsgeschehen, entstehen vor $\mathrm{Ort}^{34}$, ebenso könnten kommunale Gesundheitslandschaften entstehen, in denen bei Beeinträchtigung passende Versorgungsstrukturen partizipativ gestaltet und verfügbar werden.

34 Lübbe/Beck, Individuelle Hilfeplanung - Anforderungen an die Behindertenhilfe, Bremen 2002; Rund/Lutz/Fiegler, Kommunale Teilhabeplanung im Kontext Integrierter Sozialraumplanung, in: Lampke/Rohrmann/Schädler (Hrsg.), Örtliche Teilhabeplanung mit und für Menschen mit Behinderungen. Theorie und Praxis, Wiesbaden 2011, S. 89-104. 
Neue kommunale Ermöglichungsräume (als „enabling communities“, befähigte und befähigende Gemeinschaften ${ }^{35}$ ) zu realisieren, wird eine Aufgabe der kommenden Jahre $\operatorname{sein}^{36}$. Dies erscheint in weiten Strecken kongruent mit dem Ziel, die Infrastruktur wohnortnah und gemeindebasiert mit den Erfordernissen einer wachsenden älteren Bevölkerung in Einklang zu bringen ${ }^{37}$. Mit Nassehi ${ }^{38}$ könnte man dafür neu fragen, nicht, „wer die Exkludierten sind, wer die Überflüssigen und wer die Ausgegrenzten. Vielleicht ist es angemessener danach zu fragen, unter welchen Bedingungen sich Lebenslagen als exkludiert, überflüssig, ausgegrenzt beschreiben.“.

In dem künftigen Gemeinwesen sollten auch beeinträchtigte Bürgerinnen und Bürger selbstbestimmt leben und dabei in die Gemeinschaft einbezogen sein (Article 19 UNBRK „Living independently and being included in the community“). Allen müssen die gleichen Wahlmöglichkeiten offenstehen, die dafür notwendigen wirksamen und geeigneten Vorkehrungen sind $\mathrm{zu}$ treffen (,take effective and appropriate measures to facilitate full enjoyment by persons with disabilities of this right and their full inclusion and participation in the community"). Dabei ist an Elemente gedacht wie

- die Wahl des Aufenthaltsortes, der Wohnform und der Personen, mit denen man lebt (a),

- Zugang zu Diensten in der Gemeinde (auch als persönliche Assistenz) (ohne Risiken der Isolation und Aussonderung) (b),

- Dienste für alle in der Gemeinde, die den Bedürfnissen der Gleichberechtigung bei Behinderung Rechnung tragen (c).

Wie solche Transformationen trotz teilweise konträrer Dynamiken einzelner jahrzehntelang etablierter Leistungserbringer und -anbieter aus Altenhilfe, Eingliederungshilfe, Kinder- und Jugendhilfe, Pflegesicherung etc. und regionaler sowie überregionaler Wirkkräfte gelingen können, wird aufwändig zu konzipieren und wissenschaftlich zu prüfen sein, wie dies auch von Beginn des Transformationsprozesses von Sach- zu Geldleistungssystemen an geschah (Monitoring der Entwicklung und Umsetzung des Persönlichen Budgets nach § 17 SGB IX $\left.{ }^{39}\right)$.

35 Treber/Haas, Enabling Community. Gemeinwesen zur Inklusion befähigen, in: Schütte (Hrsg.), Abschied vom Fürsorgerecht. Von der „Eingliederungshilfe für behinderte Menschen“ zum Recht auf soziale Teilhabe, Münster, Hamburg \& London 2011, S. 141, 142.

36 Kreutz/Lachwitz/Trenk-Hinterberger, Die UN-Behindertenrechtskonvention in der Praxis. Erläuterungen der Regelungen und Anwendungsgebiete, Köln 2013; Steinhart, Teilhabe für alle im Quartier. Herausforderungen für die Sozialpsychiatrie, in: Aktion Psychisch Kranke/Weiß (Hrsg.), Psychiatriereform 2011 ... Der Mensch im Sozialraum, Bonn 2012, S. 52-68; Welti, Rechtliche Grundlagen einer örtlichen Teilhabeplanung, in: Lampke/Rohrmann/Schädler (Hrsg.) (Fußn. 34), S. 55-67.

37 Barth, Örtliche Teilhabeplanung für ältere Menschen mit und ohne Behinderung, in: Lampke/Rohrmann/Schädler (Hrsg.) (Fußn. 34), S. 257-268.

38 Nassehi (Fußn. 31), S. 68.

39 Schäfers/Wacker/Wansing, Persönliches Budget im Wohnheim, Wiesbaden 2009; Wacker, Das Persönliche Budget. Neue Leistungsgestaltung in der Behindertenhilfe, Die Rehabilitation 48/2009, S. 4-14; Wansing, Persönliches Budget, in: Greving (Hrsg.), Kompendium Heilpädagogik. Bd. 2, Troisdorf 2007, S. 165-175. 


\section{Ausblick - evidenzbasierte Maßstäbe für Leistungssysteme als Königsweg?}

Die Verwebungen und Verflechtungen bei Beeinträchtigung und Behinderung auf der Landkarte der Lebenslagen und Lebenswelten sind vermutlich relevant und kaum weniger komplex und herausfordernd als die Netze und mehrdimensionalen Verbindungen, die systembiologisch mit Hilfe von Hochleistungscomputern aufgedeckt werden sollen. Zwischen molekularem Minimaß und Gesamtorganismen verbergen sich charakteristische Muster, die Leben bedeuten. Mit ebendiesen systemtheoretischen Logiken will Sozialwissenschaft der Gesellschaft auf die Spur ihrer Konstruktionen und regulatorischen Prozesse kommen.

Schon Kant betrachtete Lebewesen als ,organisierte und sich selbst organisierende Wesen" ${ }^{40}$. Hieraus ergeben sich Perspektiven und mögliche Folgen, denen sich sozialpolitisch und sozialrechtlich fokussierte Forschung nicht entziehen kann, noch kann sie sich auf einen unspezifischen Begriff der Vernetzung zurückziehen. Wie gezeigt wurde, müssen geeignete Heuristiken noch identifiziert werden, die bei den Rekonstruktionen sozialer Verflechtungen ein Abbild des Alltags ermöglichen 41 . Dies könnte Aufgabe der Teilhabeforschung sein, die Modelle entwickelt und konkrete Situationen erkennt und benennt, die Zusammenhänge zwischen Innen und Außen der Inklusion und Exklusion, ihrem Wesen und ihrer Erscheinung, aufdeckt, beschreibt und prüft. Als ein Indikator kann die ICF-Systematik wertvolle Anhaltspunkte bereitstellen, obwohl Alltag vielfach komplexe Feinstrukturen enthält.

Widerstände sind zu erwarten, weil sich Gesellschaft durch Exklusion auch funktionsfähig hält und lokale Strukturen kein Allheilmittel sind. Einerseits wird vor kommunalen „Ghettos ohne Mauern“ für multipel und kumuliert Benachteiligte gewarnt ${ }^{42}$, andererseits die Nicht-Unterscheidbarkeit tatsächlicher Leistungsansprüche gegenüber gesellschaftspolitischen Desideraten sozialer Kontextbezüge als ,,aus der Flasche gelassene[r] Geist" heraufbeschworen ${ }^{43}$.

Diese Einwände mögen als Hinweis dienen für manche Mühsal, mit der Prozesse zur Implementierung bzw. Transformation gerechter Lebenschancen für behinderte Menschen im Gemeinwesen verbunden sind ${ }^{44}$. Konkrete Maßstäbe im Prozess des evidenz-

40 Zit. nach Rheinberger, Biologie - mit System neu definiert, Max Planck Forschung H. 1/2012, S. 12-16.

41 Cordts, Örtliche Teilhabeplanung als Herausforderung für vertikale und horizontale Kooperationsprozesse beteiligter Leistungsträger und Anbieterorganisationen, in: Lampke/Rohrmann/Schädler (Hrsg.) (Fußn. 34), S. 111-126.

42 Häußermann, Die Krise der „sozialen Stadt“. Warum der sozialräumliche Wandel der Städte eine eigenständige Ursache für Ausgrenzung ist, in: Bude/Willisch (Hrsg.) (Fußn. 31), S. 294, 311.

43 Luthe, Begriff und Gegenstand des Rehabilitationsrechts, SGb 8/07, S. 454, 461.

44 Etzioni, Die Entdeckung des Gemeinwesens - Das Programm des Kommunitarismus, Frankfurt a.M. 1998. 
basierten Monitoring und Vergleichens wären hilfreich, jenseits allen guten Willens. Stein 45 bezeichnet Inklusion ,als Utopie, aber nicht im Sinne eines Unerreichbaren, sondern als U-topos: als Noch-nicht-Ort“". Wegweisungen können aus der systematischen Analyse von Ausgrenzungsprozessen und bei der Fahndung nach Kraftquellen und Möglichkeitsräumen aufscheinen. Dies ist eine gesellschaftliche Frage und Aufgabe, bei der sowohl individuelle Unterstützungsbedarfe eingelöst werden, als auch nicht aussondernde gesellschaftliche Strukturen entstehen.

Für eine im immer neuen Übergang erforderliche praktische Utopie der Teilhabe von behinderten Menschen, wird ein Handeln im Optativ den Weg bahnen, das von Schütz angedachte phantasievolle Durchspielen von Möglichkeiten ${ }^{46}$, das zu einer Anpassung der bestehenden und sich entwickelnden Entwürfe im Handlungsvollzug führt. Somit wäre die Differenz zwischen dem, was wir dank unserer Vorstellungskraft vom Umgang mit Behinderung erwarten und dem, was geschieht, Quelle von Neuem. Das Neue entsteht in der Transformation des Alten. Wie viel Licht hier am Ende des Tunnels sein wird, muss sich finden!

\section{Literatur}

Antonovsky, Salutogenese: zur Entmystifizierung der Gesundheit (Dt. erw. Hrsg. Franke). DGVT, Tübingen 1997.

Aselmeier, Community Care und Menschen mit geistiger Behinderung, Wiesbaden 2008.

Baer/Bittner/Göttschke, Mehrdimensionale Diskriminierung - Begriffe, Theorien und juristische Analyse. Teilexpertise im Auftrag der Antidiskrimimierungsstelle des Bundes, Berlin 2011.

Bandura, Social cognitive theory: An agentic perspective, Annual Review of Psychology 52/2001, S. $1-26$.

Barth, Örtliche Teilhabeplanung für ältere Menschen mit und ohne Behinderung, in: Lampke/Rohrmann/Schädler (Hrsg.), Örtliche Teilhabeplanung mit und für Menschen mit Behinderungen. Theorie und Praxis, Wiesbaden 2011, S. 257-268.

Bingel, Sozialraumorientierung revisited. Geschichte, Funktion und Theorie sozialraumbezogener Sozialer Arbeit, Wiesbaden 2011.

Bertschi, Im Dazwischen von Individuum und Gesellschaft. Topologie eines blinden Flecks der Soziologie, Bielefeld 2010.

Bude/Lantermann, Soziale Exklusion und Exklusionsempfinden, Kölner Zeitschrift für Soziologie und Sozialpsychologie 58/2006, S. 233-252.

45 Stein, Integration als Möglichkeitsraum der Vergesellschaftung von Individuen, Behindertenpädagogik 47/2008, S. 283, 288.

46 Knoblauch, Alfred Schütz, die Phantasie und das Neue. Überlegungen zu einer Theorie des kreativen Handelns, in: Schröer/Bidlo (Hrsg.), Die Entdeckung des Neuen. Qualitative Sozialforschung als Hermeneutische Wissenssoziologie, Wiesbaden 2011, S. 99, 105. 
Council of the European Union. Joint report by the Commission and the Council of social inclusion. Brussels 2004, p 10, SOC 115 ECOFIN 80 EDUC 46 SAN 49.

Cordts, Örtliche Teilhabeplanung als Herausforderung für vertikale und horizontale Kooperationsprozesse beteiligter Leistungsträger und Anbieterorganisationen, in: Lampke/Rohrmann/Schädler (Hrsg.), Örtliche Teilhabeplanung mit und für Menschen mit Behinderungen. Theorie und Praxis, Wiesbaden 2011, S. 111-126.

Dern/Inowlocki/Oberlies, Mehrdimensionale Diskriminierung - Eine empirische Untersuchung anhand von autobiographisch-narrativen Interviews. Teilexpertise im Auftrag der Antidiskriminierungsstelle des Bundes, Berlin 2011.

Etzioni, Die Entdeckung des Gemeinwesens - Das Programm des Kommunitarismus. Fischer, Frankfurt a.M. 1998.

Europäischer Kongress von Menschen mit Behinderung, Die Madrid-Deklaration. 23. März 2002, Madrid. Disability World. A bimonthly web-zine of international disability news and views Issue no. 15 September-October 2002. http://www.disabilityworld.org/0910_02/news/madrid.shtml (Zugegriffen: 02. Januar 2013).

Früchtel/Budde/Cyprian, Sozialer Raum und Soziale Arbeit. Textbook: Theoretische Grundlagen, Wiesbaden 2007.

Gaedt, Einrichtungen für Ausgeschlossene oder „Ein Ort zum Leben“. Überlegungen zur Betreuung Geistigbehinderter, Jahrbuch für kritische Medizin Bd. 7, Berlin 1981, S. 96-109.

Grüber, Disability Mainstreaming, IMEW konkret Nr. 10, Dezember 2007, abrufbar unter: http://www.imew.de/index.php?id=237 (letzter Zugriff: 19.03.2011).

Häußermann, (2006) Die Krise der ,sozialen Stadt“. Warum der sozialräumliche Wandel der Städte eine eigenständige Ursache für Ausgrenzung ist, in: Bude/Willisch (Hrsg.), Das Problem der Exklusion. Ausgegrenzte, Entbehrliche, Überflüssige, Hamburg 2006, S. 294313.

Hinte, Eigensinn und Lebensraum - zum Stand der Diskussion um das Fachkonzept „Sozialraumorientierung“, VHN 78/2009, S. 20-33.

Kessl/Reutlinger, Sozialraum, in: Otto/Thiersch (Hrsg.), Handbuch Soziale Arbeit, 4. Aufl. München/Basel 2011, S. 1508-1516.

Knoblauch, Alfred Schütz, die Phantasie und das Neue. Überlegungen zu einer Theorie des kreativen Handelns, in: Schröer/Bidlo (Hrsg.), Die Entdeckung des Neuen. Qualitative Sozialforschung als Hermeneutische Wissenssoziologie, Wiesbaden 2011, S. 99-116.

Kreutz/Lachwitz/Trenk-Hinterberger, Die UN-Behindertenrechtskonvention in der Praxis. Erläuterungen der Regelungen und Anwendungsgebiete, Köln 2013.

Kronauer, „Exklusion“ als Kategorie einer kritischen Gesellschaftsanalyse. Vorschläge für eine anstehende Debatte, in: Bude/Willisch (Hrsg.), Das Problem der Exklusion. Ausgegrenzte, Entbehrliche, Überflüssige, Hamburg 2006, S. 27-45.

Lübbe/Beck, Individuelle Hilfeplanung - Anforderungen an die Behindertenhilfe, Bremen 2002.

Luthe, Begriff und Gegenstand des Rehabilitationsrechts, SGb 2007, S. 454-467.

Lightfood, Community-based rehabilitation. A rapidly growing method for supporting people with disabilities, Social Work 47/2004, S. 455-468.

Lutz/Wenning, Differenzen über Differenz - Einführung in die Debatten, in: Lutz/Wenning (Hrsg.), Unterschiedlich verschieden. Differenz in der Erziehungswissenschaft, Opladen 2001, S. 11-24. 
Minow, Making All the Difference. Inclusion, Exclusion and American Law, Ithaka/New York 1990.

Nassehi, Die paradoxe Einheit von Inklusion und Exklusion. Ein systemtheoretischer Blick auf die „Phänomene“, in: Bude/Willisch (Hrsg.), Das Problem der Exklusion. Ausgegrenzte, Entbehrliche, Überflüssige, Hamburg 2006, S. 46-69.

Rat der Europäischen Union, Ziele bei der Bekämpfung der Armut und der sozialen Ausgrenzung (Dokument 14164/1/02 REV 1), Brüssel 2002.

Rund/Lutz/Fiegler (2011). Kommunale Teilhabeplanung im Kontext Integrierter Sozialraumplanung, in: Lampke/Rohrmann/Schädler (Hrsg.), Örtliche Teilhabeplanung mit und für Menschen mit Behinderungen. Theorie und Praxis, Wiesbaden 2011, S. 89-104.

Rheinberger, Man weiß nicht genau, was man nicht weiß. Über die Kunst, das Unbekannte zu erforschen, NZZ, 5.5.2007.

Rheinberger, Biologie - mit System neu definiert, Max Planck Forschung H. 1 2012, S. 12-16.

Schäfers/Wacker/Wansing, Persönliches Budget im Wohnheim, Wiesbaden 2009.

Scholz, Die Entwicklung des Community Care-Ansatzes in Deutschland und Großbritannien im Vergleich, Norderstedt 2009.

Schütz, Der sinnhafte Aufbau der sozialen Welt. Eine Einleitung in die verstehende Soziologie, Frankfurt a.M. 1974.

Schütz/Luckmann, Strukturen der Lebenswelt, Bd. I, Frankfurt a.M. 1979.

Schuntermann, Einführung in die ICF: Grundkurs, Übungen, offene Fragen, Hamburg 2005.

Sen, Ökonomie für den Menschen, München 2007.

Sennett, Respekt im Zeitalter der Ungleichheit, Berlin 2004.

Steinhart, Teilhabe für alle im Quartier. Herausforderungen für die Sozialpsychiatrie, in: Aktion Psychisch Kranke/Weiß (Hrsg.), Psychiatriereform 2011 ... Der Mensch im Sozialraum, Bonn 2012, S. 52-68.

Stein, Integration als Möglichkeitsraum der Vergesellschaftung von Individuen, Behindertenpädagogik 47/2008, S. 283-298.

Treber/Haas, Enabling Community. Gemeinwesen zur Inklusion befähigen, in: Schütte (Hrsg.), Abschied vom Fürsorgerecht. Von der „Eingliederungshilfe für behinderte Menschen“ zum Recht auf soziale Teilhabe, Münster/Hamburg/London 2011, S. 141-161.

UN - United Nations, The Standard Rules on the Equalization of Opportunities for Persons with Disabilities. Adopted by the United Nations General Assembly, forty-eighth session, resolution 48/96, annex, of 20 December 1993. http://www.un.org/ga/search/view_doc.asp? symbol=A/RES/48/96\&Lang=E\&Area=RESOLUTION. Zugegriffen: 02. Januar 2013.

Voges/Jürgens/Mauer/Meyer, Methoden und Grundlagen des Lebenslagenansatzes, ZeS: Zentrum für Sozialpolitik Endbericht 2003 (http://www.soziologie.uni-kiel.de/bergersozun/ Voges_Lebenslagenansatz.pdf).

Wacker, Behinderung in der Gesellschaft. 50 Jahre im soziologischen Blick - vom Dialog zum Diskurs, Geistige Behinderung 47/2008, S. 42-61.

Wacker, Das Persönliche Budget. Neue Leistungsgestaltung in der Behindertenhilfe, Die Rehabilitation 48/2009, S. 4-14. 
Wacker/Wetzler/Metzler/Hornung, Leben im Heim. Angebotsstrukturen und Chancen selbständiger Lebensführung in Wohneinrichtungen der Behindertenhilfe, Schriftenreihe des BMG 102, Baden-Baden 1998.

Wacker, Behindertenpolitik, Behindertenarbeit, in: Otto/Thiersch (Hrsg.), Handbuch Soziale Arbeit, 4. Aufl. München/Basel 2011, S. 87-100.

Wansing, Persönliches Budget, in: Greving (Hrsg.), Kompendium Heilpädagogik, Bd. 2, Troisdorf 2007, S. 165-175.

Wansing, Der Inklusionsbegriff in der Behindertenrechtskonvention, in: Welke (Hrsg.), UNBehindertenrechtskonvention mit rechtlichen Erläuterungen, Berlin 2012, S. 93-103

Welti, Rechtliche Grundlagen einer örtlichen Teilhabeplanung, in: Lampke/Rohrmann/Schädler (Hrsg.), Örtliche Teilhabeplanung mit und für Menschen mit Behinderungen. Theorie und Praxis, Wiesbaden 2011, S. 55-67.

Weisser, Sozialraumorientierung und Situationen der Behinderung - Über die sozialräumliche Strukturierung von Abhängigkeitsbeziehungen, VHN 79/2010, S. 4-10.

WHO - World Health Organization, International Classification of Functioning, Disability, and Health (ICF), Geneva 2001. Dt.: DIMDI - Deutsches Institut für Medizinische Dokumentation und Information (Hrsg.), Internationale Klassifikation der Funktionsfähigkeit, Behinderung und Gesundheit, Genf 2005, http://www.dimdi.de/static/de/klassi/icf/index.htm (Zugegriffen: 02. Januar 2013).

Young, Justice and the Politics of Difference, Princeton/New Jersey 1990.

Zinsmeister, Mehrdimensionale Diskriminierung. Das Recht behinderter Frauen auf Gleichberechtigung und seine Gewährleistung durch Art. 3 GG und das einfache Recht, BadenBaden 2007. 


\title{
Inklusionsorientierung im Sozialraum - Verpflichtung und Herausforderung
}

\author{
Clemens Dannenbeck
}

I. Einführung - Der Inklusionsdiskurs

II. Feuilletonistische Randbemerkungen aus dem beschädigten Leben oder (fast) wie im richtigen Leben

1. Die Grenzen der Inklusion in der inklusiven Praxis 50

2. Ramponierte Teilhabe - der vermessene inklusive Sozialraum $\quad 50$

3. Einsicht in die eigene Verstricktheit 51

4. Inklusion einfach machen - und alles wird gut 51

III. Wissenschaftlicher Teil $\quad 51$

IV. Ausblick $\quad 56$

\section{Einführung - Der Inklusionsdiskurs}

Im Folgenden wird versucht, die Notwendigkeit der stärkeren Berücksichtigung einer sozialräumlichen Perspektive in der politischen und öffentlichen Inklusionsdebatte zu begründen, um anschließend in Ansätzen herauszuarbeiten, zu welchen behindertenpolitischen Blickverschiebungen eine solche Fokussierung des Sozialraums führen würde. Ausgangspunkt der Argumentation ist folgende Wahrnehmung des Inklusionsdiskurses, wie er sich infolge der Ratifizierung der UN-BRK durch die Bundesrepublik Deutschland seit dem Jahr $2009^{1}$ präsentiert: Die Ratifizierung der UN-BRK führte zu einem (für manche durchaus überraschend) hohem Handlungsdruck und -bedarf auf diversen politischen Entscheidungsebenen, nicht zuletzt durch die mit der freiwilligen Ratifizierung des Fakultativprotokolls verbundenen inhärenten Kontroll- und Qualitätsmechanismen, die die Reaktionen und Umsetzungsprozesse national wie international beglei-

1 Die UN-Konvention über die Rechte von Menschen mit Behinderung ist seit dem 26. März 2009 in der Bundesrepublik Deutschland rechtsverbindlich. Grundlage ist das Gesetz zum Übereinkommen der Vereinten Nationen vom 13. Dezember 2006 über die Rechte von Menschen mit Behinderungen sowie das Fakultativprotokoll vom 13. Dezember 2006 zum Übereinkommen der Vereinten Nationen über die Rechte von Menschen mit Behinderungen (Deutsch, Englisch, Französisch); vgl. hierzu: http://www.institut-fuer-menschenrechte.de/de/monitoring-stelle.html (Stand 10. Juni 2012). 
ten und fortgesetzter öffentlicher Beobachtung und Kritik aussetzen. Folge davon sind Aktionen, Absichtserklärungen und Beteuerungen, die sich zum Teil in verstärkten integrationspolitischen Bemühungen ${ }^{2}$, zum Teil aber auch in einer inflationären Inklusionsrhetorik ${ }^{3}$ niederschlagen. Beispielhaft für die semantische Übernahme bei gleichzeitiger inhaltlicher Aushöhlung der Forderung nach voller selbstbestimmter gesellschaftlicher Teilhabe für alle Menschen ist zum einen die Übersetzungsgeschichte der deutschsprachigen Fassung des Textes der UN-BRK ${ }^{4}$, zum anderen etwa die Umbenennung des so genannten bayerischen Weges der Integration durch eine Vielfalt schulischer Angebote zur Inklusion durch eine Vielfalt schulischer Angebote 5 . Die Konvention ist aber nicht nur Diskursanlass, sondern auch geltendes Recht ${ }^{6}$. In der inflationären Inklusionsrhetorik spiegelt sich der systematische Versuch, von der gesamtgesellschaftlichen Dimension der Herausforderung durch Inklusion abzulenken und den umfassenden Anspruch, der sich aus dem Geist der UN-BRK ableiten lässt, auf eine alleinige Aufgabe bisweilen des Bildungssystems, bisweilen auch nur von Sonderschulen, möglicherweise sogar zur Sache einzelner pädagogischer Lehrkräfte zu reduzieren. Einem solchermaßen politisch verkürzten Verständnis von Inklusion entspricht unverkennbar eine Strategie der Individualisierung des Problems: Inklusion darf, kann und soll doch jeder - ob in Schule oder Betrieb - „einfach machen“7, zumal - so die stets beifalls-

2 Zum Stand der Dinge vgl. den Ersten Staatenbericht der Bundesrepublik Deutschland zum Übereinkommen der Vereinten Nationen über Rechte von Menschen mit Behinderungen, vom Bundeskabinett beschlossen am 3. August 2011, abrufbar unter: http://www.bmas.de/SharedDocs/Downloads /DE/staatenbericht-2011.pdf?_blob=publicationFile (Stand 10. Juni 2012) sowie den Nationalen Aktionsplan der Bundesregierung zur Umsetzung der UN Behindertenrechtskonvention vom August 2011, abrufbar unter: http://www.bmas.de/SharedDocs/Downloads/DE/PDF-Publikationen/a740nationaler-aktionsplan-barrierefrei.pdf?_blob=publicationFile (Stand 10. Juni 2012).

3 Vgl. hierzu den Beitrag von Wocken, Über die Entkernung der Behindertenrechtskonvention. Ein deutsches Trauerspiel in 14 Akten, mit einem Vorspiel und einem Abgesang, Inklusion-online, Zeitschrift für Inklusion Nr. 4 2011, abrufbar unter http://www.inklusion-online.net/index.php/ inklusion/article/view/139/135 (Stand 10. Juni 2012).

4 Vgl. hierzu die Schattenübersetzung der UN-BRK durch Netzwerk Artikel 3: http://www.netzwerkartikel-3.de/index.php?view=article\&id=93:international-schattenuebersetzung （Stand 10. Juni 2012).

5 Vgl. das gleichnamige Konzeptpapier des Bayerischen Staatsministeriums für Unterricht und Kultus vom 1. August 2011 IV.6 - S 8040.5.1 - 4a.107922 zur Umsetzung der UN-Behindertenrechtskonvention in Bayern hinsichtlich des Gesetzentwurfs zur Änderung des Bayerischen Gesetzes über das Erziehungs- und Unterrichtswesen - Umsetzung der UN-Behindertenrechtskonvention im bayerischen Schulwesen (Inklusion) (BT-Drucks. 16/8100 vom 28.03.2011) - dazu siehe auch: http://www.verwaltung.bayern.de/portal/cl/1058/Gesamtliste.html?cl.document=4036315 (Stand 10. Juni 2012).

6 So die bündige Formulierung von Hirschberg in ihrem Beitrag auf der Fachtagung der Informations- und Beratungsstelle Studium und Behinderung (IBS) des Deutschen Studentenwerks (DSW) am 15. Juni 2012 in Berlin.

7 So das Motto zur Dachkampagne des Bundesministeriums für Arbeit und Soziales zur Umsetzung der UN Behindertenrechtskonvention und Namensgeberin der Konferenz „Inklusion einfach machen - von Bildung über Ausbildung in Arbeit" vom 7. Dezember 2011. 
trächtige Formel - Inklusion schließlich im Kopf beginnt. In einer derartigen strategischen Ausrichtung werden Ressourcen- und Haltungsaspekte gegeneinander ausgespielt. Im Übrigen ist leider auch zu beobachten, dass die Fachdisziplinen der Integrations- und Inklusionspädagogik im Eifer des Gefechts und angesichts des momentan in der inklusionspolitischen Morgendämmerung verspürten Rückenwinds keineswegs davor gefeit sind, dieses (meiner Ansicht nach verhängnisvolle) Spielchen mitzuspielen: Da schießen so genannte und selbst ernannte „Inklusionsschulen“ wie Pilze aus dem Boden und jeder kleine Integrationsfortschritt wird mittlerweile als Mosaiksteinchen einer sich am Horizont bereits mehr oder weniger deutlich abzeichnenden inklusiven Gesellschaft gefeiert. Der breiten Öffentlichkeit bleibt dabei nur eines, nämlich sich die neue politisch korrekte Leitvokabel anzueignen - was bis gestern Integration hieß, wird fürderhin Inklusion genannt - ansonsten scheinen die besten Voraussetzungen mittlerweile geschaffen, dass nun endlich wieder Ruhe (etwa in Gestalt des viel zitierten Schulfriedens) einkehrt im Lande ${ }^{8}$.

Menschen mit Behinderung selbst (oder im Falle von Kindern deren Eltern) kommen in diesem Prozess zwar eher zu Wort - den Möglichkeiten, ihre ambivalenten und nicht selten kritischen Erfahrungen mit dem Umsetzungsprozess aber wirksam zu Gehör verschaffen, sind nach wie vor jedoch enge Grenzen gesetzt. Überparteiliche und interfraktionelle Einigkeit im moralisch Gebotenen wirkt hier als neue Barriere, die einer Verschiebung von Repräsentationschancen in den Weg gestellt ist. Für akut Betroffene ändert sich zunächst wenig bis gar nichts. Hier und da kann der eine oder andere zusätzliche Integrationserfolg verzeichnet werden ${ }^{9}$ - aber von dem oft beschworenen Perspektivwechsel sind wir nach wie vor weit entfernt. Eine zukünftige Biografieforschung über in Zeiten der Inklusionsrhetorik aufgewachsene Menschen wird ergeben, dass diese Generation nur mit unerheblich weniger Teilhabebarrieren zu kämpfen hatte als die Generationen davor ${ }^{10}$. Die Provokation der UN-BRK droht im Strohfeuer von „ein bisschen mehr Integration, soweit und sofern wir uns das in finanzpolitischen Krisenzeiten leisten können“", zu verpuffen.

8 So ein Originalkommentar auf dem Bildungskongress 2011 der CSU an der Hochschule Landshut.

9 So erhöhen die 41 in diesem Schuljahr in Bayern inthronisierten Schulen mit dem Schulprofil Inklusion zwar die Integrationsstatistik - gleichzeitig aber bleibt der Separationsanteil trotz insgesamt sinkender Schüler/innenzahlen tendenziell erstaunlich konstant. Integration und Separation werden so nicht zueinander ausschließenden Gegensätzen, sondern zu einer systemtheoretisch logischen Konsequenz eines fortgesetzt gewollten Nebeneinanders von Regel- und Sondereinrichtungen.

10 Vgl. hierzu Dorrance, Barrierefrei vom Kindergarten in die Schule? Eine Untersuchung zur Kontinuität von Integration aus der Sicht betroffener Eltern, Reihe Forschung, Bad Heilbrunn 2010; sowie Bruner, KörperSpuren. Zur Dekonstruktion von Körper und Behinderung in biografischen Erzählungen von Frauen, Bielefeld 2005. 


\section{Feuilletonistische Randbemerkungen aus dem beschädigten Leben oder (fast) wie im richtigen Leben}

\section{Die Grenzen der Inklusion in der inklusiven Praxis}

K. besucht eine Inklusionsschule. Er ist Inklusionsschüler. Seit Kurzem. I-Schüler war er schon immer. K. bereichert die Statistik. Mit und Dank ihm steigt die Inklusionsrate. Bei Problemen wendet er sich seit dem Schuljahr 2011/12 an seinen Inklusionslehrer. Der ist nunmehr zum Inklusionsbeauftragten anvanciert. Als solcher kann er stolz über die mittlerweile erreichte Inklusionsquote berichten. Das Soll ist erfüllt - natürlich stellt es eine Übertreibung dar, dass im kommenden Schuljahr eine Inklusionsecke der Begegnung im Pausenhof eingerichtet werden soll, die zu besuchen sich dann übrigens auch für Ali lohnen würde, dessen I-Bereitschaft ja bislang noch zu wünschen übrig lässt.

Tatsache ist aber, dass die Grenzen der Inklusion wohl irgendwo in oder vor Moskau liegen müssen. Man kennt ja diese heftigen Wintereinbrüche im Osten - das ist auch der Grund, weshalb K., dem I-Rollstuhlfahrer, eine Teilnahme an der gemeinschaftsbildenden Klassenfahrt in die Fremde leider auch in Inklusionszeiten nicht ermöglicht werden kann - oder haben Sie schon einmal einen Rollstuhl bei minus 20 Grad durch meterhohe Schneewehen geschoben (oder sich durch dieselben schieben lassen?) - das ist dem stärksten Bufti nicht zuzumuten und würde im Übrigen nun wirklich den allgemeinem Klassenfortschritt hemmen.

\section{Ramponierte Teilhabe - der vermessene inklusive Sozialraum}

„Für solche wie Euch haben WIR doch extra diese Rampen eingebaut" flucht der MVG-Busfahrer, sich die öffentlichen Investitionen in den inklusiven Sozialraum vergegenwärtigend, als er bemerkt, dass sich der Rollstuhlfahrer K. mit seiner Begleitung unversehens und (womöglich auch) vorschriftswidrig des öffentlichen Raums selbstbestimmt, also unter Missachtung der zur Verfügung stehenden Rampe bemächtigt. Die Wege der Inklusion im Sozialraum sind schließlich vorgezeichnet und deutlich markiert - und die Teilhabe am Öffentlichen Nahverkehr führt in Deutschland nun mal vorzugsweise über Rampen. Schließlich dient das auch der Sicherheit der anderen Fahrgäste sowie der eigenen. Also: Die Normalen nehmen die normalen Eingänge. Der Rest benutzt die Resterampe. Noch stationenweit ist dem städtischen Busführer der Ärger über die augenscheinliche Fehlinvestition seiner Steuergelder deutlich ins Gesicht geschrieben. 


\section{Einsicht in die eigene Verstricktheit}

„Antithese - Für den, der nicht mitmacht, besteht die Gefahr, dass er sich für besser hält als die anderen und seine Kritik der Gesellschaft missbraucht als Ideologie für sein privates Interesse. Während er danach tastet, die eigene Existenz zum hinfälligen Bilde einer richtigen zu machen, sollte er dieser Hinfälligkeit eingedenk bleiben und wissen, wie wenig das Bild das richtige Leben ersetzt. Solchem Eingedenken aber widerstrebt die Schwerkraft des Bürgerlichen in ihm selber. Der Distanzierte bleibt verstrickt wie der Betriebsame, vor diesem hat er nichts voraus als die Einsicht in seine Verstricktheit und das Glück der winzigen Freiheit, die im Erkennen als solchem liegt."11

\section{Inklusion einfach machen - und alles wird gut}

Bayern an der Spitze der Bewegung: „Inklusion durch Kooperation“ statt „Integration durch Kooperation" - Resultat eines Updates der ministeriellen Homepage 12. Vollzugsmeldung: „München-Land ist schon inklusiv!“ - jüngst registrierte selbstbewusste Aussage von Seiten einer Schulamtsvertretung ${ }^{13}$. Vorsichtiger Optimismus: „München wird inklusiv!“ - Motto der Aktivitäten zur Erstellung eines städtischen Aktionsplans für die Landeshauptstadt ${ }^{14}$. Ein Gespenst geht um im Land: Wir werden jetzt mal schnell inklusiv. Alles wird gut.

\section{Wissenschaftlicher Teil}

Welche Konsequenzen ergeben sich aus einem inklusionsorientierten Blick auf den Sozialraum - bzw. aus einer sozialräumlichen Interpretation der Orientierung an Inklusion? Das Thema ist längst entdeckt: So lassen sich unschwer Veranstaltungsreihen finden, die sich mit „Sozialraum und Inklusion“ befassen ${ }^{15}$. Ebenso existieren Empfehlun-

11 Adorno, Minima Moralia. Reflexionen aus dem beschädigten Leben, Frankfurt a.M. 2001, S. 30 f.

12 Vgl. hierzu die Präsentation von MR Weigl vom Bayerischen Staatsministerium für Unterricht und Kultus: http://www.schulamt.mm.unterallgaeu.de/fileadmin/dateien/aktuelles/inklusion_durch_ kooperation_auftakt_folien_28_10_2009.pdf (Stand 10. Juni 2012).

13 Mündliche Aussage, getroffen auf dem Fachtag „Inklusion“ des Kreisjugendrings München-Land am 4. Mai 2012 im Rahmen des KJR-Jahresthemas 2012 und 2013 „Mädchen und Jungen mit Behinderung ins Blickfeld nehmen - Inklusion als Aufgabe für den KJR München-Land“.

14 Zum aktuellen Stand vgl. http://www.muenchen-wird-inklusiv.de/ (Stand: 10. Juni 2012).

15 Bspw. die gleichnamige Reihe, veranstaltet von der Europäischen Akademie für Inklusion: http://www.inklusion-sh.eu (Stand 10. Juni 2012). 
gen für den inklusiven Sozialraum, etwa des Deutschen Vereins für öffentliche und private Fürsorge e.V. ${ }^{16}$ Dort heißt es:

„I. Inklusion als gesellschaftliche Herausforderung

Wir leben in einer pluralistischen Gesellschaft, in der Menschen mit und ohne Behinderungen, alte und junge Menschen, mit oder ohne Migrationshintergrund gemeinsam Lebensräume nutzen und gestalten. Ein solidarisches Miteinander, in dem jede/r sein Leben individuell und selbstbestimmt in jeder Lebensphase - mal jung, mal alt, mal mit mehr Einschränkungen, mal mit weniger - gestalten kann, setzt gegenseitige Wertschätzung und die Erkenntnis voraus, dass sich jede/r gewinnbringend in die Gemeinschaft einbringen kann. Gelingt Inklusion nicht, sind Vernachlässigungen und Ausgrenzungen zu befürchten. Reparaturarbeiten zum Aufholen missglückter Inklusion sind aufwendig und ggf. kostspielig. Gelingt Inklusion, wird die Gesellschaft durch ihre Vielfalt bereichert, besteht weniger Anpassungsdruck für jede/n und wird Solidarität gelebt. Eine inklusive Herangehensweise an Angebote des öffentlichen Raums insgesamt und des Sozialen im Besonderen vermindert Doppelarbeit, schafft Synergien und steigert damit die Effizienz der Angebote. Unterschiedliche Angebote für verschiedene Zielgruppen können durch eine inklusive Ausrichtung aller Angebote ggf. zusammengeführt werden. Auch ist davon auszugehen, dass in inklusiven barrierefreien Lebensräumen nicht alle, aber einige spezialisierte Angebote entbehrlich werden.

$[\ldots]^{\text {“ }}$

Ich versuche den Sozialraum im Folgenden als einen Ort zu begreifen, an dem und in dem sich Inklusionsdiskurse manifestieren - handlungspraktisch, strukturell und rhetorisch. Insofern ist (vielleicht einzig) der Blick auf den Sozialraum geeignet, um eine diskurstheoretisch informierte Analyse von Inklusions- und Exklusionsverhältnissen durchzuführen und aufrecht zu erhalten. Deren Sinn bestünde darin, die Einlösung der normativen Forderung nach voller selbstbestimmter gesellschaftlicher Teilhabe, wie sie aus der UN-BRK abzuleiten ist, am Leben und Erleben aller im Sozialraum Agierenden zu messen.

Was wäre unter Inklusion zu verstehen, wenn aus ihr eine andere Praxis folgen soll als die, welche sich im Namen von Integrationsbemühungen für Menschen mit Behinderung und Menschen mit Migrationshintergrund in den letzten Jahrzehnten herausgebildet hat? Meine These: In diesem Sinne inklusive Entwicklungen, die den Namen verdienen, wären in dem Maße zu vermelden, in dem strukturelle Veränderungen zum Abbau vorhandener oder zur Verhinderung von neu errichteter Teilhabebarrieren führen und diese Prozesse von (institutionellen oder personellen) Entscheidungsträgern gestaltet und vollzogen werden, die ihr soziales Handeln einer anhaltenden Reflexion in Bezug auf die darin enthaltenen Inklusions- und Exklusionspotenziale unterziehen. Haben wir es mit informierten Inklusionshaltungen zu tun, die nicht in Strukturveränderungen eingebettet sind, so mag das wünschens- und allemal lobenswert sein (und auch manchem 'Betroffenen' vereinzelt zugutekommen), jedoch handelt es sich dabei lediglich

16 Vgl. hierzu http://www.deutscher-verein.de/05-empfehlungen/alteraltenhilfe/Eckpunkte_fuer_ einen_inklusiven_Sozialraum (Stand 10. Juni 2012). 
um Entwicklungen in einem integrationslogischen Kontext. Auch die umgekehrte Konstellation ist zu beobachten: Zusätzliche Ressourcen mögen zu verbesserten Rahmenbedingungen für den Umgang mit Vielfalt beitragen, allein die professionellen Einstellungen der Akteure, möglicherweise auch die Hartnäckigkeit wirksamer Vorurteile mögen einer Inklusionspraxis, die diesen Namen verdient, nach wie vor entgegenstehen. Inklusion so verstanden, beginnt nicht einerseits im Kopf und hängt andererseits vom politischen Willen ab - Inklusion bemisst sich vielmehr am Zustand der Gesellschaft insgesamt. Damit geraten einige Annahmen in den Fokus, die im Inklusionsdiskurs bisweilen zwar anklingen, selten aber in ihrer Konsequenz zu Ende gedacht werden:

Inklusive Prozesse erweisen sich nicht an einzelnen Best-Practice-Beispielen, Modellversuchen oder Leuchtturmprojekten - so begrüßenswert diese auch als exemplarische Belege für gelingende integrative Praxis sein mögen; diese entsprechen nicht dem, was unter einer Inklusionsorientierung zu verstehen wäre, nämlich der politische Wille, gesellschaftliche Teilsysteme in Bezug auf ihre Exklusionsdynamik fortgesetzt auf den Prüfstand zu stellen - im Sinne einer reflexiv begriffenen Gestaltungsaufgabe des Gemeinwesens in öffentlicher Verantwortung. Aus der Sicht so genannter Betroffener und das können wir bekanntlich alle sein, in je unterschiedlichen biografischen Konstellationen des Lebenslaufs - ist das Erleben des barrierefreien und Teilhabe ermöglichenden Sozialraums der Maßstab für Inklusionsqualität - und nicht etwa das eventuelle Vorhandensein einer Schwerpunktschule mit dem Schulprofil Inklusion oder ähnliches. Die Kategorie der Behinderung ebenso wie die der Barrierefreiheit verliert dabei ihre Tauglichkeit zur Unterscheidung von Zielgruppen. Sozialraumbezogene Inklusionsprozesse manifestieren sich demnach nicht in einer (oft notwendigen und wünschenswerten) Statusverbesserung Benachteiligter oder Diskriminierter, sondern sie manifestieren sich im Abbau und in der Verhinderung von Diskreditierungs- und Diskriminierungspotenzialen, die mit den gesellschaftlich bedeutsamen bzw. bedeutsam gemachten Differenzen wie Gender, Herkunft, Alter, sexuelle Orientierung, kultureller Identität oder den ökonomischen Verwertungspotenzialen individuellen Leistungsvermögens verknüpft werden. Insofern verschiebt sich im Rahmen von sozialraumorientierten Inklusionsbemühungen gewissermaßen der Fokus des Blicks - sozialraumorientierte Inklusionsbemühungen erweisen sich somit nicht in der Etablierung zusätzlicher Pilotprojekte, in denen die Bedingungen eines ,gelingenden Umgangs mit Vielfalt“ erprobt werden, sondern in Praxen, die sich theoretisch an intersektionalen Fragestellungen orientieren - z.B. in einer Querschnittsanalyse der Wirksamkeit rassistischer Logiken im Sozialraum, auch innerhalb eines Diskurskontextes, der durch positive Konnotationen von kultureller Differenz gekennzeichnet ist. Insbesondere Mecheril ${ }^{17}$ weist in diesem Zusammenhang darauf hin, dass dies etwa auch zur Verfestigung der Annahme von statischen Kulturen führen kann. Insofern sind kulturanthropologische Annahmen kultureller Differenz und die soziale Wirksamkeit kultureller Prägungen

17 Mecheril, Migrationspädagogik, Weinheim, Basel, Berlin 2010. 
stets aufeinander und miteinander bezogen. Ein weiteres Beispiel sozialraumorientierter Inklusionsbemühungen würde die institutionalisierte Bearbeitung der Frage nach sozioökonomischen Ungleichheitsentwicklungen darstellen, die sich im Sozialraum abbilden und die Bedingungen des Zusammenlebens bestimmen. Dabei geht es im Rahmen einer intersektionalen Perspektive stets um die Frage der Wechselwirkungen zwischen den gesellschaftliche Bedeutsamkeit erlangenden Differenzmerkmalen.

Es ist jeweils die unauflösbare Ambivalenz des Spannungsfelds von Vielfalt und Differenz, das die zentrale Bedingung für sozialraumorientierte Inklusionsbemühungen darstellt. Eine einseitige Auflösung dieser Ambivalenz zugunsten der Anerkennung von Vielfalt führt zur folkloristischen Überhöhung des „Alles so schön bunt hier“, die blind für die reale Wirksamkeit normierender Machtverhältnisse oftmals in einer Bereicherungsrhetorik verharrt - die Auflösung der unhintergehbaren Ambivalenz zugunsten einer Fokussierung auf die Kritik von Differenzverhältnissen übersieht hingegen nicht selten die realexistierende Vielfalt vom Blickwinkel einer verallgemeinernden Identitätsposition aus, die vor allem den der eigenen Position inhärenten hegemonialen Deutungsanspruch nicht kritisch zu reflektieren vermag.

Bereits bei Prengel ${ }^{18}$ deutete sich an, dass im pädagogischen Diskurs mit Blick auf Geschlecht, Kultur und Behinderung jeweils ähnliche Verlaufsmuster der Betrachtung von Vielfalt zu beobachten waren und sind. Man könnte die Genese der fachlichen Blickrichtungen davon ausgehend als eine Entwicklung von Defizit- über Differenz- zu Bereicherungsparadigmen beschreiben, wobei sich die Diskurse nicht jeweils notwendigerweise parallel und in gleicher Intensität und Geschwindigkeit entwickelten. Insbesondere im heutigen handlungspraktisch ausgerichteten pädagogischen Inklusionsdiskurs, der sich auf die Konsequenzen beruft, die sich aus der UN-BRK ergeben, scheint das Bereicherungsparadigma erst punktuell kritisch hinterfragt ${ }^{19}$. Eine Position der Kritik ist jedoch - blickt man auf die benachbarten Gender- und Kulturdiskurse - in dem Maße zu erwarten, in dem Menschen mit Behinderung selbst sich zunehmend in der Lage sehen, ihre Repräsentationspotenziale zu nutzen. Das allerdings hätte zur Folge, dass sich die Definitionsmacht über Form, Inhalt und Gestalt von zukünftigen Inklusionsprozessen gewissermaßen selbst vervielfältigt - und ggf. mancher selbsternannten Kampfinstanz für Inklusion unversehens zwischen den Fingern zerrinnt.

Ein - außerhalb Bayerns - erprobtes und verbreitetes Selbstevaluationsinstrument zur Gestaltung kommunaler Inklusionsentwicklung ist der kommunale Index für Inklusion. Es handelt sich um eine Übersetzung und Adaption des von Booth und Ainscow

18 Vgl. Prengel, Pädagogik der Vielfalt. Verschiedenheit und Gleichberechtigung in Interkultureller, Feministischer und Integrativer Pädagogik. Schule und Gesellschaft, Bd. 2, Wiesbaden 2006.

19 Vgl. dazu bspw. Rathgeb, Disability Studies. Die Perspektive beeinträchtigter Menschen. Reihe: Perspektiven kritischer Sozialer Arbeit, Wiesbaden 2012; sowie Anhorn u.a., Kritik der Sozialen Arbeit - kritische Soziale Arbeit. Perspektiven kritischer Sozialer Arbeit, Wiesbaden 2012. 
(2002) ${ }^{20}$ zuerst entwickelten Index for Inclusion ${ }^{21}$, der mittlerweile neben der deutschen Übersetzung von Boban/Hinz 22 auch in einer speziellen Fassung für Kindertageseinrichtungen ${ }^{23}$ vorliegt. Im kommunalen Index für Inklusion geht es um „Diversität in der Entwicklung eines inklusiven Gemeinwesens" ${ }^{\text {24 }}$. Kommune wird dabei - im tendenziellen Unterschied zu Ansätzen kommunaler Teilhabeplanung 25 , die auch explizit auf Menschen mit Behinderung zielen - nicht nur als lokale Verwaltungseinheit begriffen, sondern als

„große Gemeinschaft: In ihr leben Menschen zusammen, in vielen Formen und auf vielen Ebenen. Hier können Menschen im Austausch mit anderen Menschen und der Verwaltungsebene ihres Ortes gemeinsam wirksam werden" 26 .

Diese an Vielfalt orientierte Perspektive schließt folgende Aktivitätsebenen der prozesshaften Inklusionsentwicklung ein:

- die Kommune als Wohn- und Lebensort;

- die inklusive Entwicklung kommunaler Organisationen;

- die Kooperation und Vernetzung innerhalb der Kommune.

Die Arbeit mit dem Kommunalen Index für Inklusion verspricht:

- Verbesserungspotenziale zu identifizieren und realisierbare Schritte zeitnah umzusetzen;

- durch organisationsübergreifende Aktivitäten auf kommunaler Ebene Synergieeffekte zu erreichen;

- das gesellschaftliche Bewusstsein zur Wertschätzung von Unterschiedlichkeit zu erhöhen;

20 Booth/Ainscow, Index for Inclusion, Developing Learning and Participation in Schools. Center for Studies on Inclusive Education, 3. Aufl. London 2011.

21 Montag Stiftung Jugend und Gesellschaft (Hrsg.), Kommunaler Index für Inklusion. Arbeitsbuch. Montag Stiftung, Köln 2010, abrufbar unter: http://www.montagstiftungen.com/fileadmin/

Redaktion/Jugend_und_Gesellschaft/PDF/Projekte/Kommunaler_Index/KommunenundInklusion_ Arbeitsbuch_web.pdf (Stand 10. Juni 2012).

22 Boban/Hinz, Index für Inklusion - Lernen und Teilhabe in der Schule der Vielfalt entwickeln, Halle 2003, http://www.eenet.org.uk/resources/docs/Index\%20German.pdf (Stand 10. Juni 2012).

23 Gewerkschaft Erziehung und Wissenschaft (GEW), Index für Inklusion (Tageseinrichtungen für Kinder. „Lernen, Partizipation und Spiel in der inklusiven Kindertageseinrichtung entwickeln“. Deutschsprachige Ausgabe. http://www.fhoow.de/sowe//downloads/12/index_veranstaltung_ emden.pdf (Stand 10. Juni 2012).

24 Lawrenz, Diversität in der Entwicklung eines inklusiven Gemeinwesens: Erfahrungen mit dem „Kommunalen Index für Inklusion“, Archiv für Wissenschaft und Praxis der Sozialen Arbeit. Diversity Management und soziale Arbeit 1/2012, S. 83-91.

25 Vgl. hierzu etwa die Arbeiten von Rohrmann, http://www.buergergesellschaft.de/fileadmin/pdf/ gastbeitrag_rohrmann_100430.pdf (Stand 10. Juni 2012) und Lampke/Rohrmann/Schädler, Örtliche Teilhabeplanung mit und für Menschen mit Behinderungen: Theorie und Praxis, Wiesbaden 2011.

26 Imhäuser, in: Montag Stiftung Jugend und Gesellschaft (Hrsg.), Inklusion vor Ort - Der Kommunale Index für Inklusion - Ein Praxishandbuch, Berlin 2011, S. 8. 
- Selbstwirksamkeit im direkten Lebensumfeld zu erfahren und zu gestalten;

- barrierefreie Beteiligung an der Gestaltung des kommunalen Zusammenlebens und -wirkens zu verbessern;

- Dienstleistungen kommunaler und anderer Träger an Bedürfnis und Bedarf der Bevölkerung anzupassen.

Ansatzpunkt für die Initiierung, Kontrolle und kontinuierliche Fortentwicklung des Inklusionsprozesses ist dabei der einzelne handelnde Akteur. Ihre verändernde Wirkung entfalten fünf voneinander unterscheidbare Ebenen der Kommune primär über die auf gutem Willen, Motivation und „richtiger“ Einstellungen basierende Praxis handelnder Subjekte. „Andere Menschen mit der Bedeutung von Inklusion 'infizieren', die Idee weitertragen und weitere Aktive und Multiplikator/-innen gewinnen, ist ein zentraler Aspekt von inklusiven Prozessen“27. Die inklusive Entwicklung nimmt dabei ihren Ausgangspunkt in einer reflexiven Versicherung eines angemessenen Inklusionsverständnisses auf personaler/individueller Ebene, markiert dann den Sozialraum (begriffen als „Nachbarschaftsebene“) in Gestalt alltäglicher Begegnungen und setzt sich dann fort auf der Ebene von Organisationen, deren Vernetzung bis hin zur kommunalen/politischen Ebene, die im Sinne einer ,großen inklusiven Gemeinschaft“ beschrieben wird. Kern inklusiver Prozesse ist dabei Partizipation: „Inklusion als Schlüssel für eine zukunftsfähige Gesellschaft kann nur in partizipativen Prozessen erreicht werden, in denen die Menschen mitgestalten, ihre Selbstwirksamkeit erfahren und Verantwortung übernehmen können“ 28 .

\section{Ausblick}

Diesem ohne Zweifel praxisorientierten und praxisbewährten Ansatz zu Initiierung, Aufrechterhaltung und Fortentwicklung integrativer Prozesse wohnt ebenso unübersehbar ein normativer und moralischer Impetus inne, der - so meine These - geeignet ist, vom kritischen Potenzial der Inklusionsforderung, wie sie in der UN-BRK aufscheint, eher abzulenken. Der Ansatz, Exklusions- und Inklusionsverhältnisse im Sozialraum quasi von unten strukturell verändern zu wollen, ohne diese Verhältnisse gleichzeitig einer strukturellen Analyse und reflexiven Kritik zu unterziehen, muss zwangsläufig und bestenfalls in Leuchtturmprojekten enden, was durchaus auch zu einer punktuell vorbildhaften ,inklusiven Gemeinwesenentwicklung“ 29 beitragen kann. Der nachhaltige Erfolg dieser Impulse bleibt jedoch abhängig von der geteilten Motivationslage und der

27 Lawrenz (Fußn. 24).

28 Lawrenz (Fußn. 24).

29 Vgl. hierzu etwa die inklusiven Entwicklungen in der Gemeinde Wiener Neudorf, dokumentiert auf: http://www.wiener-neudorf.gv.at/system/web/zusatzseite.aspx?menuonr=218605890\&detailonr= 218608690 (Stand 10. Juni 2012). 
Verständigung darüber, wie Vielfalt und deren anerkennende Wahrnehmung jeweils begrifflich gefasst werden. Da eine systematische Analyse der gesellschaftlichen Verhältnisse, auf denen Integrationsprozesse beruhen, nicht vorgesehen ist, erscheinen diese methodischen Ansätze auch wenig gegen politische Umarmungs- und Instrumentalisierungsstrategien gewappnet zu sein. „Ob Inklusion nachhaltig umgesetzt werden kann, wird sich maßgeblich darin zeigen, inwieweit sich die Wertschätzung von Vielfalt und die Umsetzung inklusiver Werte im täglichen Miteinander widerspiegeln“30. Möglicherweise stellt die in der Neuausgabe des Index for Inclusion ${ }^{31}$ erfolgte Weiterentwicklung des methodischen Ansatzes eine Konsequenz aus seiner theoretischen Unterkomplexität dar. Hier scheinen über die kommunikativ-handlungspraktisch begriffenen „inklusiven Werte“ hinaus Aspekte in die Betrachtung einbezogen, die Anknüpfungspunkte für gesellschaftskritische Perspektiven bieten könnten.

Die transdisziplinäre Herausforderung für eine wissenschaftliche Befassung mit dem Umsetzungsprozess der UN-BRK liegt darüber hinaus in der Analyse der Strukturen des herrschenden Inklusionsdiskurses und der nicht zuletzt auch in ihm enthaltenen und sich spiegelnden Machtverhältnisse. Wer spricht warum und in welcher Absicht von Inklusion (und nicht von Integration) - und mit welchen Folgen? Wie verändert der herrschende Inklusionsdiskurs die Wahrnehmungen von Vielfalt, die Herstellungspraxen von Differenz(en)? Für welche Diskurse erweist sich der Inklusionsdiskurs als anschlussfähig und wo bzw. wie werden diese Anschlüsse hergestellt (und wo nicht)? Welche Entwicklungen werden angestoßen, wenn etwa die Debatte um Interkulturelle Öffnung sozialer Einrichtungen sich mit der „benachbarten“ Diskussion um ein inklusives Bildungssystem zu verständigen sucht? Welches Schicksal erfahren hierarchisierte Differenzen in der Gesellschaft angesichts von Diversityorientierung und Mainstreamingstrategien? Welche Effekte hat die „Entdeckung von Vielfalt“ in und durch eine Pädagogik, die sich selbst als inklusiv begreift? Und zu guter Letzt: Verlieren dabei Behinderung, kulturelle Herkunft, Genderzugehörigkeit, sexuelle Orientierung oder Alter etwas von ihren Exklusionspotenzialen, ihrer Diskreditierbarkeit?

Vielleicht ist aber Inklusion schlussendlich eben auch keine „Lösung“ - zumindest nicht der Beleg für ein Stück Richtiges im Falschen - sondern lediglich ein weiterer Begründungzusammenhang für den Auftrag zur Gestaltung einer menschengerechteren Welt und der Notwendigkeit, sich mit den gegebenen Verhältnissen nicht zufriedenzugeben.

30 Lawrenz (Fußn. 24).

31 Vgl. auch Booth, Wie sollen wir zusammen leben? Inklusion als wertbezogener Rahmen für die pädagogische Praxis, in: Gewerkschaft Erziehung und Wissenschaft (Hrsg.), Jugendhilfe und Sozialarbeit, 2. Auflage Frankfurt a.M. 2011. 
II. Barrieren der Inklusion und Perspektiven ihrer Überwindung 


\title{
Inklusionsbarrieren im Sozialraum
}

\author{
Markus Dederich
}

$\begin{array}{ll}\text { I. Einleitung } & 61\end{array}$

II. Sozialraumorientierung und spezialisierte Hilfe $\quad 62$

III. Zur ästhetischen Dimension sozialer Barrieren im Sozialraum 63

IV. Anerkennung als Modus der Inklusion $\quad 66$

$\begin{array}{ll}\text { V. Literatur } & 67\end{array}$

\section{Einleitung}

In diesem Beitrag wird es darum gehen, ein übergeordnetes, am ehesten kulturwissenschaftlich zu fassendes Problem bei der Umsetzung der Sozialraumorientierung zu fokussieren. Dieses besteht im Kern darin, dass die Schaffung inklusiver Lebensräume nicht allein durch eine politisch beschlossene und sozialtechnologisch umzusetzende Restrukturierung der Hilfe zu bewerkstelligen ist. Die Verwirklichung des inklusiven Anspruchs der Sozialraumorientierung erfordert vielmehr auch einen tiefgreifenden kulturellen Wandel. Dieser Wandel müsste im Kern darauf hinauslaufen, dass es neben der Zuerkennung von Rechten und der Neuorganisation der Hilfe zu einer Wertschätzung behinderter Menschen und anderer marginalisierter Gruppen kommen muss. Dieser Wandel wird häufig im Kontext von Anerkennungsethiken thematisiert.

Vor diesem Hintergrund werde ich nachfolgend die These skizzieren, dass genau dieses Fehlen der Zuerkennung eines positiven Werts behinderter Menschen ein zentrales Hindernis bei der Verwirklichung von Inklusion im Sozialraum ist. Dass Menschen mit Behinderung weitgehend immer noch nicht als gleichwertige Mitbürgerinnen und Mitbürger wahrgenommen und akzeptiert werden, hat zahlreiche Gründe. Ich werde mich nachfolgend darauf beschränken einen wichtigen, bis heute aber im Diskurs so gut wie nicht wahrgenommenen Grund herauszuarbeiten: Die ästhetische Dimension von Behinderung. 


\section{Sozialraumorientierung und spezialisierte Hilfe}

Ich beginne mit einigen Anmerkungen dazu, was ich nachfolgend unter „Sozialraumorientierung" verstehe. Der soziale Raum ist die Lebenswelt, der sinnhaft aufgebaute Ort der täglichen Lebensvollzüge der Individuen und ihrer sozialen Beziehungen. Er ist das Feld, in dem die soziale Welt in ihrer differenzierten Gestalt anschaulich wird, in dem Beziehungsnetze geknüpft, Nähe und Distanz ausgehandelt und in der Folge Zugehörigkeiten und Nicht-Zugehörigkeiten erfahren werden. Im Sozialraum werden soziale Differenzierungen in Form feiner Unterschiede verkörpert. Die prägende und normierende Kraft von Institutionen wird hier ebenso konkret erlebt wie das Ausfechten von Verteilungskämpfen und die Durchsetzung von Interessen.

In der Behindertenhilfe und Sozialen Arbeit steht Sozialraumorientierung für den Versuch, die traditionsreiche Verbesonderung von Hilfen und durch Hilfen rückgängig zu machen und Inklusionsräume zu schaffen. Im Fokus des Konzepts steht nicht das für sich betrachtete Individuum mit seinen Problemen, Defiziten, Schädigungen und Beeinträchtigungen, sondern das jeweilige konkrete Lebensumfeld, in dem das Individuum lebt. Dabei wird davon ausgegangen, dass spezifische Probleme und daraus resultierende Unterstützungsbedarfe von Menschen nicht nur durch eher abstrakte gesellschaftliche Rahmenbedingungen, etwa das Recht und die Ökonomie, bestimmt werden, sondern durch konkrete Lebensumstände im Quartier.

Die Behindertenhilfe und Soziale Arbeit gäbe es zumindest in der bekannten Form nicht, wenn alle soweit inkludiert wären, dass die vorhandenen Regelsysteme auch behinderte Menschen adressieren könnten, d.h. auch jene, die in ihrem Wahrnehmen, Denken und Handeln anders oder eingeschränkt sind und deshalb über das normale Maß oder auf andere Weise als üblich unterstützungsbedürftig sind. Eine Grundidee der Sozialraumorientierung ist, die Hilfen zu den Menschen zu bringen und nicht umgekehrt. Hierzu müssen legitime besondere Bedarfe der Individuen (und d.h. immer auch: ihre Defizite) identifiziert und entsprechende Ressourcen zugewiesen werden. Jedoch steht dieses bisher übliche Verfahren in einer eigentümlichen Spannung zu einer Grundidee der Sozialraumorientierung: Es ist die Spannung zwischen einer klaren Individuumszentrierung und dem gleichzeitigen Versuch, diese zu überwinden. Dieses Verfahren birgt zudem das Problem, dass eine Legitimation der Zuweisung zusätzlicher Hilfen auf der Identifikation von Defiziten beruht und daher einem Grundgedanken des Empowerments, dem Ausgehen von den Stärken, der Tendenz nach zuwiderläuft.

So schreibt Hinte: „Unsere Finanzierungsstränge setzen das diagnostizierte und immer neu diagnostizierte Defizit voraus und sie orientieren Fachkräfte wie Leistungsberechtigte darauf, immer wieder die vermeintlichen Schwächen zu benennen, und das prägt mental enorm. Das gilt für die Arbeit mit Einzelnen wie mit Quartieren“1. Deshalb

1 Hinte, Sozialraumorientierung - auch ein Konzept für die Behindertenhilfe?, http://bbw.johannesdiakonie.de/pr/documents/ReferatHinte.pdf, S. 7. 
müssen Fachkräfte lernen, ,auf der Grundlage gezielter Defizitbeschreibungen immer wieder Ressourcen in benachteiligte Quartiere zu lenken bzw. zu benachteiligten Menschen zu lenken, und gleichzeitig dürfen wir uns nicht den Menschen bzw. den Quartieren gegenüber von diesen Defizitbeschreibungen chloroformieren lassen“" 2 .

Ein weiterer Effekt ist, dass die Konzentration auf bestimmte Personen oder ganze Zielgruppen durch die Ausblendung ihrer Verflochtenheit mit dem umgebenden Sozialraum zu einer Blickverengung führt, was häufig zur Folge hat, deren Besonderheit zu markieren bzw. zu bekräftigen und dadurch zu deren Isolation bzw. Marginalität beizutragen ${ }^{3}$.

\section{Zur ästhetischen Dimension sozialer Barrieren im Sozialraum}

Die Idee Inklusion lebt u.a. von der normativen Vorstellung der egalitären Differenz - die Achtung anderer Menschen, die trotz empirischer Unterschiede als wertvoll und zugehörig wahrgenommen werden. Dem steht aber die Tendenz entgegen, Menschen gerade nicht als Individuen in ihrer Einmaligkeit zu sehen, sondern sie einzuordnen, zu typisieren, als zu sozialen Gruppen gehörig wahrzunehmen und mit klaren Bewertungen zu belegen. Im Sozialraum, so meine These, herrscht nicht das Prinzip ,egalitärer Differenz'. Er lässt sich umgekehrt durch Prozesse der Herstellung und Stabilisierung wertgeleiteter Differenzen charakterisieren, d.h. durch Prozesse sozialer Grenzziehungen, die in Selbst- oder Fremdexklusion münden können.

Ein Beispiel für solche Prozesse der Grenzziehung ist die Herausbildung „gruppenbezogener Menschenfeindlichkeit", die eine Arbeitsgruppe um Wilhelm Heitmeyer seit 2002 erforscht. Ein Element der gruppenbezogenen Menschenfeindlichkeit ist die Heterophobie, die Angst vor dem, was anders ist und daher - gerade in Zeiten vielfach empfundener ökonomischer Destabilisierung und sozialer Verunsicherung - als bedrohlich empfunden wird. Dieses Element beinhaltet die Abwertung von Menschen mit Behinderung und basiert, wie die anderen Elemente auch, etwa die Islamfeindlichkeit oder die Homophobie, auf einer Ideologie der Ungleichwertigkeit ${ }^{4}$.

$\mathrm{Ob}$ für Menschen mit Behinderung offene inklusive Sozialräume entstehen, ist vor dem Hintergrund dieser Forschungen wesentlich davon abhängig, wie sie wahrgenommen werden, ob sie als unheimlich, verstörend, befremdlich usw. gelten, welcher Wert oder Unwert ihnen zugeschrieben wird. Prägnant formuliert: Sozialraumorientierung

2 Hinte, Sozialraumorientierung - auch ein Konzept für die Behindertenhilfe?, http://bbw.johannesdiakonie.de/pr/documents/ReferatHinte.pdf, S. 7 f.

3 Hinte, Sozialraumorientierung - auch ein Konzept für die Behindertenhilfe?, http://bbw.johannesdiakonie.de/pr/documents/ReferatHinte.pdf, S. 8.

4 Heitmeyer (Hrsg.), Deutsche Zustände. Folge 10, Berlin 2012. 
sorgt für Sichtbarkeit, aber Sichtbarkeit allein ist kein Garant für positive Wertschätzung oder Anerkennung und damit für Inklusion.

Zur Begründung meiner zentralen These greife ich auf eine Studie von Siebers ${ }^{5}$ zurück. Darin entwickelt er den Gedanken, dass bei allen Bemühungen um die Gestaltung einer barrierefreien Umwelt ästhetische Fragen eine zentrale Rolle spielen. Er zeigt auf, dass Bestrebungen zur Erreichung von Barrierefreiheit durch häufig unbewusst bleibende Gegenbewegungen, durch Widerstände und Abwehrmechanismen konterkariert und untergraben werden. Siebers formuliert prägnant: „Die ästhetische Aversion gegen Behinderung reicht weit über den einzelnen behinderten Körper hinaus bis zur symbolischen Präsenz von Behinderung in der gebauten Umwelt" “6.

Behinderung als ästhetisches Phänomen zu begreifen eröffnet theoretisch somit die Möglichkeit, gesellschaftliche Homogenisierungsbedürfnisse und daran anknüpfende Exklusionsmechanismen (die das Nichthomogenisierbare an den Rand drängen oder ausscheiden), produktiv zu analysieren.

Zugehörigkeit und Nichtzugehörigkeit, Vertrautheit und Fremdheit, die gemeinschaftsbildende Unterscheidung von ,Wir' und , die Anderen', kulturelle Wertvorstellungen und deren Negation werden in hohem Maße über ästhetische Prozesse geschaffen. Dies kann man gut anhand der Wirkungsmächtigkeit der (kulturell und historisch höchst varianten) Unterscheidung von ,schön' und ,hässlich' belegen. Pointiert schreibt Müller: „Schönheit wirkt anziehend und weckt Sympathien: sie entspricht der Vollendung des eigenweltlich Vertrauten. Beim Anblick von Hässlichem ist man geneigt, den Blick abzuwenden, wird irgendwie Unbehagen wach, weil wir zu glauben geneigt sind, dass die äußere Verzerrung nicht von ungefähr kommt, dass sie der Abdruck innerer ,Unstimmigkeiten', die mimische Projektion einer seelischen Entstellung ist. In allen Kulturen löst Hässlichkeit Argwohn, zumindest ungute Empfindungen aus"7.

Hier zeigt sich ein enger Zusammenhang zwischen Ästhetik und Sozialmoral: Ästhetische Phänomene werden mit Werten aufgeladen und so zur Grundlage moralisch unterfütterter oder explizit normativer Urteile über andere Menschen, die ihrerseits eng mit politischen Haltungen verbunden sind. Häufig entscheiden sie mit über Akzeptanz oder Ablehnung von Individuen oder ganzen Gruppen. Es sind also in nicht unerheblichem Maße ästhetische Faktoren, die zu erhöhten Sensibilitäten gegenüber , ordnungswidrigen Elementen' im Sozialraum führen ${ }^{8}$. Wenn Individuen oder Gruppen wegen ihres Äußeren, ihres Verhaltens, ihrer Bräuche usw. als aus der vertrauten Ordnung herausfallend erlebt werden, wird ihnen häufig eine störende oder gar bedrohliche Qualität zugeschrieben. Dies kann so weit gehen, dass sie als ,Schmutz' wahrgenommen werden,

5 Siebers, Zerbrochene Schönheit. Essays über Kunst, Ästhetik und Behinderung, Bielefeld 2009.

6 Siebers, Zerbrochene Schönheit. Essays über Kunst, Ästhetik und Behinderung, Bielefeld 2009, S. 18.

7 Müller, Der Krüppel, München 1996, S. 27.

8 Baumann, Unbehagen in der Postmoderne, Hamburg 1999. 
dessen Existenz vielfältige Gegenbewegungen zur Reinhaltung und Hygiene des politischen und sozialen Körpers in Gang setzen.

Das Handeln von Frauen, Schwulen, Lesben, Menschen mit Behinderung, Schwarzen oder anderen ethnischen Gruppen wird für krank erklärt, ,ihr Erscheinungsbild für obszön oder ekelerregend, ihr Denken für verdorben, und ihr gesellschaftlicher Einfluss wird mit einem Krebsgeschwür verglichen, das den gesunden Körper der Gesellschaft angreift"“9. Solche ästhetischen Urteile bleiben häufig unbewusst, können aber trotzdem - oder vielleicht auch gerade deshalb - eine beträchtliche politische Wirkungsmacht entfalten, denn sie prägen Vorstellungen von einem intakten, gesunden, prosperierenden Gemeinwesen.

Behinderung zeigt sich in dieser Hinsicht als doppelte Irritation: Zum einen als ästhetische Störgröße, zum anderen als Infragestellung einer kohärenten und integeren gesellschaftlichen Ordnung, einer Gesellschaft, die ,ganz' und ,heil' ist. Dem Unbehagen bzw. der Abneigung gegenüber Menschen, die als unangemessen anders eingeordnet werden, entspricht die Abscheu vor einem disharmonischen öffentlichen Raum, in dem sich die Gesellschaft und der Staat physisch und symbolisch repräsentieren.

Als das Andere der angestrebten Ordnung symbolisieren vor allem Menschen mit schweren und deutlich sichtbaren Behinderungen Unordnung, Verfall, Auflösung und Zersetzung. Daher werden sie als Angriffe auf die uns vertrauten Maßstäbe erfahren, sie stellen Konventionen und Normalitätserwartungen in Frage und drohen, diese außer Kraft zu setzen. Barrieren haben insofern eine stabilisierende Funktion und quasi hygienische Aufgabe: die Ordnung zu bewahren und öffentliche Räume ,sauber' zu halten. Wenn aber nun behinderte Menschen in höherem Maße als bisher beginnen, öffentliche Räume zu nutzen und damit Behinderung zu einem allgemein sichtbaren bzw. erfahrbaren Phänomen machen, wird diese Ordnung (zumindest zunächst, bevor eine Adaptation erfolgt) in Frage gestellt.

Vor dem Hintergrund der vorab skizzierten Überlegungen liegt es nahe die These zu formulieren, dass politisch korrekte Forderungen nach Teilhabe und Inklusion durch unbewusst bleibende Abwehrreaktionen massiv unterminiert werden. Soweit ich sehe, sind diese Zusammenhänge (mitsamt ihren nicht nur kulturellen, sondern auch politischen und ökonomischen Hintergründen) in der Inklusionsdebatte allerdings bisher theoretisch nur unzureichend gewürdigt worden.

9 Siebers, Zerbrochene Schönheit. Essays über Kunst, Ästhetik und Behinderung, Bielefeld 2009, S. 20. 


\section{Anerkennung als Modus der Inklusion}

Die soeben skizzierten Gedanken zeigen, dass ästhetische Faktoren von großer normativer, moralischer und sozialethischer Bedeutung sind. Auf diese sehr schwierige und kontroverse Diskussion kann hier nicht weiter eingegangen werden.

Jedoch möchte ich nachfolgend wenigstens einen zentralen Aspekt wieder aufgreifen, den Zusammenhang von Sichtbarkeit und Anerkennung.

Es gibt einen engen Zusammenhang zwischen sozialer Wahrnehmbarkeit und sozialer Existenz des Menschen. Eine Form, Menschen ihrer sozialen Existenz zu berauben, besteht darin, sie unsichtbar zu machen, zum Verschwinden zu bringen. In ihrer radikalsten Variante mündet diese Unsichtbarmachung in Mord, aber häufig begnügt man sich damit, Menschen aus dem öffentlichen Raum zu entfernen. Unsichtbarkeit sorgt dafür, dass sie vergessen und sozial irrelevant werden. Unsichtbarkeit bedeutet nach Honneth ${ }^{10}$ physische Nichtpräsenz und soziale Nichtexistenz und führt zu Nichtanerkennung und Demütigung. Umgekehrt kann man sagen, dass Wahrnehmbarkeit zunächst Voraussetzung dafür ist, überhaupt als Person identifiziert und erkannt zu werden. Insofern kommt der Sichtbarkeit (oder allgemeiner: der Wahrnehmbarkeit) eine sehr wichtige positive, sozialintegrative und sozialethische Funktion zu. Dies gilt zumindest, wenn die Sichtbarmachung ohne die vorab beschriebene Herstellung einer hierarchischen Ordnung auskommt, die sich beispielsweise an Kriterien wie Schönheit, öffentlicher Wohlanständigkeit oder sozialem Nutzen orientiert.

Diese Überlegung läuft darauf hinaus, dass Sichtbarkeit bzw. Wahrnehmbarkeit sich als notwendig, wenn auch nicht als hinreichend für soziale Anerkennungsprozesse erweisen, denn Anerkennung setzt Erkennen voraus, das seinerseits auch eine sinnlichästhetische Seite hat. Dies bedeutet zwar nicht, wie die Geschichte beweist, dass physische Präsenz und damit die Wahrnehmbarkeit einer Person zwangsläufig auch in Anerkennung mündet. Wie wir gesehen haben, kann Wahrnehmung, wenn sie mit Negativbewertung von Attributen des Wahrgenommenen einhergeht, zu Nichtanerkennung führen. Daher gilt: Anerkennung ist nicht bloße Wahrnehmung der anderen Person, sondern Wahrnehmung ihres positiven Wertes ${ }^{11}$.

Wie ich bereits betont habe, bedarf Anerkennung neben der Sichtbarkeit die positive Wahrnehmung des anderen. Obgleich man diese positive Wahrnehmung ethisch als unbedingt verpflichtend ansehen mag, ist es empirisch unbestritten, dass längst nicht jeder jeden anderen anerkennt. Insofern ist Anerkennung empirisch an Voraussetzungen gebunden.

10 Honneth, Kampf um Anerkennung. Zur moralischen Grammatik sozialer Konflikte, 2. Aufl. Frankfurt 2003.

11 Vgl. hierzu Honneth (Fußn. 10); kritisch: Dederich, Behinderung, Identitätspolitik und Anerkennung, Behinderte Menschen 2/2012, S. 45-54. 
Die entscheidende Frage für die Sozialraumorientierung wie für die Inklusion überhaupt wird in Zukunft sein, wie es möglich sein wird, Voraussetzungen für tatsächlich gelingende Anerkennungsprozesse zu schaffen.

\section{Literatur}

Bauman, Zygmunt, Unbehagen in der Postmoderne, Hamburg 1999.

Dederich, Markus, Behinderung, Identitätspolitik und Anerkennung, Behinderte Menschen, Heft 2/2012, S. 45-54.

Honneth, Axel, Kampf um Anerkennung. Zur moralischen Grammatik sozialer Konflikte, 2. erw. Ausgabe Frankfurt 2003.

Heitmeyer, Wilhelm (Hrsg.), Deutsche Zustände. Folge 10, Berlin 2012.

Hinte, Wolfgang, Sozialraumorientierung - auch ein Konzept für die Behindertenhilfe?, http://bbw.johannes-diakonie.de/pr/documents/ReferatHinte.pdf.

Müller, Klaus E, Der Krüppel, München 1996.

Siebers, Tobin, Zerbrochene Schönheit. Essays über Kunst, Ästhetik und Behinderung, Bielefeld 2009. 


\title{
„Mit gleichen Wahlmöglichkeiten in der Gemeinschaft leben“" Behinderungen und Enthinderungen selbstbestimmter Lebensführung
}

\author{
Gudrun Wansing
}

$\begin{array}{ll}\text { I. Einleitung } & 69\end{array}$

$\begin{array}{ll}\text { II. Inklusion, Autonomie und Lebenslagen } & 71\end{array}$

III. Inklusionsrückstände und verspätete Befreiung von Menschen mit

IV. Mit gleichen Wahlmöglichkeiten in der Gemeinschaft leben 75

V. Strukturelle Entwicklungen der wohnbezogenen Unterstützung 77

VI. Bewertungen und Wünsche aus Nutzerperspektive 79

VII. Fazit und Perspektiven der Enthinderung $\quad 82$

$\begin{array}{ll}\text { VIII. Literatur } & 85\end{array}$

\section{Einleitung}

Im Mittelpunkt des folgenden Beitrages steht Artikel 19 der UN-Behindertenrechtskonvention (im Folgenden BRK), dessen Leitperspektiven ,unabhängige Lebensführung und Einbeziehung in die Gemeinschaft (independent living and inclusion in community)“ genuin auf eine kommunale bzw. Sozialraumorientierung ausgerichtet sind.

Art. 19 Unabhängige Lebensführung und Einbeziehung in die Gemeinschaft

Die Vertragsstaaten dieses Übereinkommens anerkennen das gleiche Recht aller Menschen mit Behinderungen, mit gleichen Wahlmöglichkeiten wie andere Menschen in der Gemeinschaft zu leben, und treffen wirksame und geeignete Maßnahmen, um Menschen mit Behinderungen den vollen Genuss dieses Rechts und ihre volle Einbeziehung in die Gemeinschaft und Teilhabe an der Gemeinschaft zu erleichtern, indem sie unter anderem gewährleisten, dass

a) Menschen mit Behinderungen gleichberechtigt die Möglichkeit haben, ihren Aufenthaltsort zu wählen und zu entscheiden, wo und mit wem sie leben, und nicht verpflichtet sind, in besonderen Wohnformen zu leben; 
b) Menschen mit Behinderungen Zugang zu einer Reihe von gemeindenahen Unterstützungsdiensten zu Hause und in Einrichtungen sowie zu sonstigen gemeindenahen Unterstützungsdiensten haben, einschließlich der persönlichen Assistenz, die zur Unterstützung des Lebens in der Gemeinschaft und der Einbeziehung in die Gemeinschaft sowie zur Verhinderung von Isolation und Absonderung von der Gemeinschaft notwendig ist;

c) gemeindenahe Dienstleistungen und Einrichtungen für die Allgemeinheit Menschen mit Behinderungen auf der Grundlage der Gleichberechtigung zur Verfügung stehen und ihren Bedürfnissen Rechnung tragen.

Die folgenden Ausführungen beleuchten die Bedeutung dieses Artikels aus sozialwissenschaftlicher Perspektive ${ }^{1}$ zum einen im Kontext konzeptioneller Entwicklungen von Inklusion, Autonomie und Lebenslagen und zum anderen im Horizont empirischer Befunde zur Wohnsituation von Menschen mit Beeinträchtigungen ${ }^{2}$. Im Fokus der Ausführungen stehen Personen, die im Zusammenhang mit erheblichen, teils mehrfachen körperlichen, geistigen oder seelischen Beeinträchtigungen auf umfängliche Unterstützung zur Bewältigung ihres Alltags angewiesen sind (z.B. in den Bereichen Mobilität, Selbstversorgung, Kommunikation und soziale Beziehungen). Ihre Lebensführung bleibt in vielen Aspekten - zuweilen ein Leben lang - geprägt durch die Ambivalenz von Autonomie und Angewiesenheit. Diese Situation wird sich für viele Personen auch durch eine möglicherweise inklusive, barrierefreie Umweltgestaltung zukünftig nicht vollständig auflösen lassen, vielmehr konstituiert sich Behinderung in vielen Lebenssituationen gerade durch ein „Mehr an sozialer Abhängigkeit“3. Dieser Widersprüchlichkeit ist in einer ausgewogenen Balance zu begegnen, wie sie Rappaport bereits für das sozialpolitische Konzept des „empowerment“ formuliert hatte. Demnach „können wir nicht länger Menschen als ,Kinder in Not' oder als ,Bürger mit Rechten“ sehen, sondern vielmehr als vollwertige menschliche Wesen, die sowohl Rechte als auch Bedürfnisse haben"4.

1 Für eine rechtliche Kommentierung von Artikel 19 vgl. Banafsche, Art. 19 Unabhängige Lebensführung und Einbeziehung in die Gemeinschaft, in: Welke, UN-Behindertenrechtskonvention mit rechtlichen Erläuterungen, Berlin 2012, S. 150-163.

2 Im Folgenden wird weitgehend zwischen den Begriffen Beeinträchtigung (im Sinne funktionaler Einschränkungen) und Behinderung als sozialer Prozess und als Ergebnis der negativen Wechselwirkungen von Beeinträchtigungen und Kontextfaktoren unterschieden (vgl. die dynamischen und interaktiven Konzepte von Behinderung in der ICF und der BRK).

3 Hahn, Behinderung als soziale Abhängigkeit. Zur Situation schwerbehinderter Menschen, München 1981.

4 Rappaport, Ein Plädoyer für die Widersprüchlichkeit: Ein sozialpolitisches Konzept des „empowerment“" anstelle präventiver Ansätze, Verhaltenstherapie und psychosoziale Praxis 1985, S. 267. 


\section{Inklusion, Autonomie und Lebenslagen}

Artikel 19 greift mit der Formulierung ,,unabhängige Lebensführung ${ }^{5}$ und Einbeziehung in die Gemeinschaft" die untrennbare Verknüpfung von Autonomie und Inklusion auf. Bereits in der Präambel der BRK wird die Erkenntnis formuliert, ,wie wichtig die individuelle Autonomie und Unabhängigkeit für Menschen mit Behinderungen ist, einschließlich der Freiheit, eigene Entscheidungen zu treffen“ (Präambel, n). Ausgehend von gesellschaftlichen Modernisierungsprozessen wie der Freisetzung des Menschen aus vorgegebenen sozialen Mustern, der Individualisierung und der Pluralisierung von Lebensstilen ist das Recht auf Selbstbestimmung ein konstitutives Moment der Teilhabe an den kulturellen Errungenschaften einer demokratisch verfassten Gesellschaft.

Die Freiheit des Individuums steht keineswegs im Widerspruch zum Grundsatz der sozialen Einbeziehung (Inklusion); vielmehr stehen Autonomie und Inklusion in einer unmittelbaren Wechselwirkung zueinander. „Erst in der wechselseitigen Verwiesenheit wird klar, dass Autonomie gerade nicht die Selbstmächtigkeit des ganz auf sich gestellten Einzelnen (...) meint, sondern auf selbstbestimmtes Leben in sozialen Bezügen zielt; und im Gegenzug wird deutlich, dass soziale Inklusion ihre Qualität gerade dadurch gewinnt, dass sie Raum und Rückhalt für persönliche Lebensgestaltung bietet" 6 . Ein Leben nach eigenen Vorstellungen und Zielen führen zu können, setzt voraus, Zugang zu den verschiedenen Gesellschaftssystemen (wie Bildung, Arbeitsmarkt, Gesundheit) zu haben und (von diesen) mit relevanten Zutrittsressourcen (wie Bildungsabschlüssen, Geld, Netzwerken, politischer Macht) ausgestattet zu werden. Inklusion zielt also auf die gesellschaftlichen Voraussetzungen für soziale Teilhabe, und Autonomie bedeutet, diese strukturellen Chancen gemäß den eigenen, individuellen Lebensplänen verwirklichen zu können. In diesem Rahmen können Selbstbestimmung und Identität auch über Nicht-Zugehörigkeit und Nicht-Partizipation zum Ausdruck kommen, sofern diese selbstgewählt sind. Allerdings fallen die Freiheitsgrade der Teilhabe in den verschiedenen Gesellschaftssystemen unterschiedlich aus. So ist es in westlichen, demokratischen Gesellschaften eine höchst freiwillige Entscheidung, eine Religionszugehörigkeit zu haben oder sich für Kunst oder Sport zu interessieren. Hingegen sind die Partizipation an Bildung (über die Schulpflicht) und die Teilhabe an Erwerbsarbeit soziale Erwartungen, denen sich niemand entziehen kann. Der Zugang zu diesen Bereichen und

5 Vor dem Hintergrund der dargestellten Ambivalenz von Autonomie und Abhängigkeit wäre der Schattenübersetzung der BRK Vorzug zu geben, die den Begriff „Unabhängigkeit“ durch den Begriff „Selbstbestimmung“ ersetzt: „Selbstbestimmt Leben und Einbeziehung in die Gemeinschaft“, vgl. http://www.netzwerk-artikel-3.de/attachments/093_schattenuebersetzung-endgs.pdf.

6 Bielefeldt, Zum Innovationspotenzial der UN-Behindertenrechtskonvention. Deutsches Institut für Menschenrechte, 3. Aufl. Berlin 2009, S. 11. 
die erfolgreiche Teilhabe, im Sinne der Ausstattung mit Ressourcen, sind Voraussetzung für soziale Zugehörigkeit und Anerkennung insgesamt ${ }^{7}$.

Die wechselseitige Verbindung von Inklusion und Autonomie in der BRK liefert konzeptionelle wie empirische Anschlussstellen zum Lebenslagenansatz, der gegenwärtig (erneut) sowohl in den fachlichen Diskursen zu Behinderung und Teilhabe ${ }^{8}$ als auch für Sozialpolitik und Sozialberichterstattung 9 eine wichtige Rolle spielt. Nach Weisser, der den Lebenslagenansatz bereits in den 1950er Jahren in die Sozialpolitik eingebracht hatte, ist Lebenslage „der Spielraum, den die äußeren Umstände dem Menschen für die Erfüllung der Grundanliegen bieten, die er bei unbehinderter und gründlicher Selbstbesinnung als bestimmend für den Sinn seines Lebens ansieht"10. In der neueren Auseinandersetzung mit dem Lebenslagenansatz werden Parallelen zum Konzept der Verwirklichungschancen (,,capability approach“) von Amartya Sen diskutiert, das internationale Aufmerksamkeit erfährt ${ }^{11}$. Auch Sen betrachtet Wahlfreiheiten im Sinne von „Möglichkeiten oder umfassenden Fähigkeiten (,capabilities“) von Menschen, ein Leben führen zu können, für das sie sich mit guten Gründen entscheiden konnten und das die Grundlagen der Selbstachtung nicht in Frage stellt“12, als bedeutsam für das Wohlergehen. Gemeinsam ist dem Lebenslagenansatz, in seinen durchaus unterschiedlichen Akzentuierungen, und dem Ansatz der Verwirklichungschancen eine mehrdimensionale Perspektive auf Lebensbedingungen, welche objektive Voraussetzungen (Handlungsspielräume) und subjektive Wahrnehmungs-, Deutungs- und Bewältigungsmuster (Präferenzen, Selbstbestimmung) miteinander verbindet. Ein solcher Betrachtungsrahmen erweist sich als zielführend für eine Bewertung der Lebenssituation beeinträchtigter Menschen im Lichte der BRK und hierauf bezogene sozialpolitische Interventionen.

7 Wansing, Inklusion in einer exklusiven Gesellschaft - oder: wie der Arbeitsmarkt Teilhabe behindert, Behindertenpädagogik 2012, S. 381-396.

8 Beck/Greving, Lebenslage und Lebensbewältigung. Enzyklopädisches Handbuch der Behindertenpädagogik 5, Stuttgart 2012.

9 Engels, Lebenslagen, in: Maelicke (Hrsg.), Lexikon der Sozialwirtschaft, Baden Baden 2008, S. 643-646. Hornberg/Schröttle, Vorstudie zur Neukonzeption des Behindertenberichts, in: Bundesministerium für Arbeit und Soziales (Hrsg.), Forschungsbericht 408/2012, http://www.bmas.de/ DE/Service/Publikationen/Forschungsberichte/Forschungsberichte- Teilhabe/ $\mathrm{fb}$-fb408-vorstudie-zur-neukonzeption-des-behindertenberichtes.html.

10 Leßmann, Lebenslagen und Verwirklichungschancen (capability) - Verschiedene Wurzeln, ähnliche Konzepte, Vierteljahresschrift zur Wirtschaftsforschung 75, 2006, S. 33.

11 Ebd.

12 Sen, Ökonomie für den Menschen. Wege zur Gerechtigkeit und Solidarität in der Marktwirtschaft, München, Wien 2000, S. 29. 


\section{Inklusionsrückstände und verspätete Befreiung von Menschen mit Be- einträchtigungen}

Erst seit dem Übergang zum 21. Jahrhundert gelangt (in Deutschland) verstärkt ins politische Bewusstsein, dass die Lebenslagen von Menschen mit Beeinträchtigungen vielfach durch Missachtung ihrer Rechte und mehrdimensionale Risiken sozialer Benachteiligung und Ausgrenzung geprägt sind ${ }^{13}$. Mit vielen Jahrzehnten Verspätung gemessen an der allgemeinen Inklusionsdynamik moderner Wohlfahrtsstaaten im Zuge der Ausweitung bürgerlicher, politischer und sozialer Rechte und ihres „Postulats einer Vollinklusion aller Menschen" 14 - wurden sie überhaupt erst als Bürgerinnen und Bürger mit gleichen Rechten wahrgenommen ${ }^{15}$. Und erst im Zuge der Anerkennung ihrer uneingeschränkten Ansprüche auf Inklusion, auf soziale Zugehörigkeit, auf Selbstbestimmung und soziale Teilhabe werden faktische „Inklusionsrückstände“16 in Form von Benachteiligung und sozialer Ausgrenzung, Diskriminierung und Fremdbestimmung, oder anders formuliert: Behinderungen ihrer sozialen Teilhabe, überhaupt erst wahrnehmbar und als mögliche Verletzung von Rechten begründungsbedürftig. Zuvor war man über einen sehr langen Zeitraum davon ausgegangen, dass Behinderung ein individuelles Schicksal bzw. ein persönliches Problem von Menschen ist, die aufgrund von Vererbung, Krankheit oder Unfall körperlich, seelisch oder geistig von einer ,normalen “ Gesamtbevölkerung abweichen. Soziale Benachteiligungen der Betroffenen wurden in diesem stark biologisch geprägten Erklärungsmodell als unabänderliche Folge ihrer individuellen Defizite gesehen. Interventionen richteten sich entsprechend auf einzelne Personen und ihre Therapie, Förderung und Betreuung bzw. auf ihre Verwahrung und Versorgung in abgesonderten Lebensräumen. Kontextfaktoren blieben dabei weitgehend unberücksichtigt. Wichtige Impulse für eine neue, rechtebasierte und auf die soziale Dimension fokussierte Sichtweise auf Behinderung gingen seit den 1970er, 80er Jahren wesentlich von der organisierten Behindertenbewegung und ihrer Forderung nach Selbstbestimmung, Selbstvertretung und der Abschaffung von Sondereinrichtungen aus. Sie machte darauf aufmerksam, dass die benachteiligte Lebenssituation von Menschen mit Beeinträchtigungen nicht naturgegeben und keine unabänderliche Folge persönlicher Defizite ist, sondern wesentlich durch soziale Faktoren bedingt ist, welche sie an

13 Für einen internationalen Vergleich siehe WHO, World Report on Disability, Genf 2011.

14 Luhmann, Die Gesellschaft der Gesellschaft, Bd. 2, Frankfurt a.M. 1999, S. 630.

15 Auf der Linie ,der evolutionären Dynamik moderner Gesellschaften“ (Burzan, Das Publikum der Gesellschaft. Inklusionsverhältnisse und Inklusionsprofile in Deutschland, Wiesbaden 2008, S. 27), die zu einer Ausweitung der Zugangsrechte und ihrer faktischen Umsetzung führt, lassen sich auch die amerikanische Bürgerrechtsbewegung der Schwarzen in den 50er und 60er Jahren sowie die Emanzipationsbewegung der Frauen in den 70er Jahren des 21. Jahrhunderts in Anlehnung an Parsons als ,nachholende Modernisierung“ beschreiben (ebd.).

16 Burzan (Fußn. 15), S. 27. 
der Ausübung ihrer Grundrechte wie Freiheit, Privatheit und Selbstbestimmung behindern.

Diese gesellschaftspolitische Dimension von Behinderung und Teilhabe wurde jedoch in der Entwicklung der deutschen Behindertenhilfe lange Zeit - und wird es zum Teil noch heute - ausgeblendet. Dabei waren wichtige politische und fachliche Impulse bereits seit den 1950er Jahren vom Normalisierungsprinzip ausgegangen, das im Kontext skandinavischer Sozialpolitik entwickelt worden war. Vor dem Hintergrund der scharfen Kritik am biologistischen Menschenbild und der Anstaltsverwahrung mit ihren menschunwürdigen Lebensbedingungen, insbesondere für Menschen mit geistiger Behinderung, folgte man hier dem Grundsatz ,to create existence for the mentally retarded as close to normal living conditions as possible"17. Es ist interessant, sich die Formulierungen des Juristen und Verwaltungsbeamten Bank-Mikkelsen (der das Normalisierungsprinzip in die dänische Sozialgesetzgebung eingebracht hatte) im Lichte der BRK noch einmal im Wortlaut anzuschauen: „This is normalization; equality with other citizens without categorizing groups"18. Mit diesem politischen Richtungswechsel von besonderen Programmen für als, behindert' bezeichnete Bevölkerungsgruppen hin zur Gewährleistung gleichberechtigter Lebensbedingungen für alle Bürgerinnen und Bürger wurden wichtige Grundsätze der BRK bereits vorweggenommen. Während die Leitperspektive Normalisierung jedoch in Skandinavien in Verbindung mit der dortigen Bürgerrechtstradition nachhaltige sozialpolitische Reformen bis hin zur Auflösung von Sondereinrichtungen nach sich zog, blieb die Umsetzung in Deutschland im Wesentlichen auf die Weiterentwicklung des professionellen Hilfesystems und seiner Institutionen beschränkt ${ }^{19}$. Dabei war die Neugestaltung im Wesentlichen durch fachliche Vorstellungen von ,normalen' Lebensbedingungen geleitet. Standards für Wohnqualität wurden vielfach ohne die wirksame Partizipation ${ }^{20}$ der Menschen mit Beeinträchtigungen entwickelt und richteten sich an einer unterstellten Homogenität einer Gruppe der Behinderten und an deren vermeintlich kollektiven Unterstützungsbedarfen aus.

Erst im Zuge einer „,verspäteten Befreiung“21 von Menschen mit Beeinträchtigungen und dem Einzug der Leitlinie Selbstbestimmung in die Unterstützungssysteme der Be-

17 Bank-Mikkelsen, in: Flynn/Nitsch (Hrsg.), Normalization, social integration, and community services, Baltimore 1980, S. 51-70.

18 Bank-Mikkelsen (Fußn. 17), S. 62.

19 Gleichwohl zum Beispiel Thimm auch für Deutschland grundlegende Reformansätze für eine gemeindeorientierte Behindertenhilfe formuliert hatte: Thimm, Leben in Nachbarschaften. Hilfen für Menschen mit Behinderungen, Freiburg 1994; ders., Tendenzen gemeinwesenorientierter Hilfen Gesellschaftliche Ausrichtungen und fachliche Konsequenzen, in: ders. (Hrsg.), Das Normalisierungsprinzip. Ein Lesebuch zu Geschichte und Gegenwart eines Reformkonzeptes, Marburg 2005.

20 Als wirksam erweist sich praktizierte Partizipation dann, wenn sich die subjektiven Erfahrungen, Interessen und Wünsche der beeinträchtigten Menschen auch faktisch auf die Gestaltung der Wohnund Lebensbedingungen auswirken und sich ihre Mitwirkung nicht in einer formalen Scheinbeteiligung bzw. bloße physische Anwesenheit in Planungsprozessen erschöpft.

21 Waldschmidt, Selbstbestimmung als Konstruktion. Alltagstheorien behinderter Frauen und Männer, 2. Aufl. Wiesbaden 2012, S. 32 ff. 
hindertenhilfe zu Beginn der 1990er Jahre wurde die subjektive Perspektive der Adressaten deutlich gestärkt. Neue Methoden der individuellen Hilfeplanung wurden eingeführt, die Position von Heimbeiräten gestärkt und Nutzerzufriedenheit avancierte zu einem wichtigen Indikator von Ergebnisqualität der sozialen Einrichtungen und Dienste. Dennoch blieben und bleiben die Grundsätze der Autonomie und Partizipation, solange sie ausschließlich im Rahmen organisierter, professioneller Dienstleistungen betrachtet werden, auf die Rolle des Konsumenten bzw. des Nutzers ${ }^{22}$ reduziert. Handlungsspielräume für Selbstbestimmung und Teilhabe werden durch eine vorgegebene Versorgungsstruktur abgesteckt und enden (bildlich gesprochen) an den Grundstücksgrenzen von Einrichtungen. So aber bleiben Optionen und Anforderungen einer kulturell üblichen und anerkannten Lebensführung begrenzt. An dieser Stelle nun liefert die UNKonvention weiterführende Impulse.

\section{Mit gleichen Wahlmöglichkeiten in der Gemeinschaft leben}

Artikel 19 der Behindertenrechtskonvention formuliert als Maßstab für die Verwirklichung einer selbstbestimmten Lebensführung „das gleiche Recht aller Menschen mit Behinderungen, mit gleichen Wahlmöglichkeiten wie andere Menschen in der Gemeinschaft zu leben“. Während im Rahmen der allgemeinen Grundsätze der BRK die abstrakte Makroebene der Gesamtgesellschaft und ihre unterschiedlichen Funktionssysteme (wie Bildung, Arbeit und Recht) als Referenzrahmen für Inklusion (,,inclusion in society“, Art. 3) zu betrachten sind ${ }^{23}$, steckt hier die „community“ (Gemeinschaft, Gemeinde) als konkreter, örtlicher und sozialer Erwartungs- und Erfahrungsraum alltäglicher Lebensführung den Betrachtungsrahmen ab. „Auf der Ebene einer Region ist der soziale Raum öffentlicher und politischer Raum der Interessensvertretung, der Sozialplanung, der Infrastrukturen, des bürgerschaftlichen Engagements, der Kultur und vieles mehr; er ist Ort der Lebensbewältigung von Menschen (Alltagsbewältigung) und räumliche Dimension ihrer sozialen Beziehungen (soziale Netzwerke), er bildet die Vermittlungsinstanz zwischen Individuum und Gesellschaft als Ort der Vergemeinschaftung, als Bürger, in Vereinen, Nachbarschaften, Freundschaftsbeziehungen"24. Einbeziehung in die Gemeinschaft meint den gleichberechtigten Zugang zu allen Aktivitäts-, Partizipationsund Kontaktspielräumen am (selbstgewählten) Wohn- und Lebensort. Die Formulierung „mit gleichen Wahlmöglichkeiten wie andere Menschen“ wirft jedoch insofern Schwie-

22 Schäfers, Lebensqualität aus Nutzersicht. Wie Menschen mit geistiger Behinderung ihre Lebenssituation beurteilen, Wiesbaden 2008; Schlebrowski, Starke Nutzer im Heim. Wirkung persönlicher Budgets auf soziale Dienstleistungen, Wiesbaden 2009.

23 Wansing, Der Inklusionsbegriff in der Behindertenrechtskonvention, in: Welke (Hrsg.), Behindertenrechtskonvention und rechtliche Erläuterungen, Berlin 2012, S. 93-103.

24 Beck/Greving, Lebenswelt, Lebenslage, in: dies. (Hrsg.), Lebenslage und Lebensbewältigung. Enzyklopädisches Handbuch der Behindertenpädagogik 5, Stuttgart 2012, S. 50. 
rigkeiten auf, als sie zum einen die Möglichkeit einer eindeutigen Differenzierung zwischen Menschen mit Beeinträchtigungen und anderen Menschen unterstellt und zum anderen von gleichen Zugangschancen der „anderen“ ausgeht. Faktisch zeigen sich aber in der Bevölkerung insgesamt höchst ungleiche Wahlmöglichkeiten in der Gemeinschaft (etwa im Zugang zu Sportvereinen oder zum Ehrenamt) in Abhängigkeit von Geschlecht, Alter, Ethnizität, Klasse oder Schicht. Zugleich sind diese Ungleichheiten auch in der Gruppe der Menschen mit Beeinträchtigungen ausgebildet. ${ }^{25}$ Diese Schwierigkeit, soziale Gruppen als Maßstab für gleiche Wahlmöglichkeiten zu bilden, wird in der BRK dadurch umgangen, dass die Entscheidungsspielräume in Artikel 19 a-c inhaltlich bestimmt werden, und zwar als a) freie Wahl des Aufenthaltsortes und der Wohnform, b) Zugang zu gemeindenahen Unterstützungsdiensten und c) Verfügbarkeit bedürfnisgerechter gemeindenaher Dienste und Einrichtungen. ${ }^{26}$ Damit werden Optionen für Selbstbestimmung und Teilhabe formuliert, die weit über bislang in Deutschland praktizierte Bewertungsmaßstäbe betreuter Wohnformen hinaus reichen, insofern hier gleiche Bedingungen im Gemeinwesen den Horizont des Möglichen und des Nötigen umreißen. Über die hier formulierten Rechte und Pflichten erfährt auch die Zielperspektive der Leistungen zur Teilhabe am Leben in der Gemeinschaft nach § 5 SGB IX eine inhaltliche Konkretisierung 27.

Zukünftige Sozialforschung und Sozialberichterstattung zu den Lebenslagen von Menschen mit Beeinträchtigungen in der Dimension alltäglicher Lebensführung und hierauf basierende regionale/örtliche Sozialplanungen werden sich an diesen Maßstäben der BRK ausrichten müssen. Hierzu bedarf es entsprechend veränderter methodologischer und methodischer Zugänge. Bislang liegen jedoch kaum aussagekräftige Daten vor, die Auskunft darüber geben könnten, inwieweit und unter welchen (persönlichen und örtlichen) Bedingungen der Anspruch auf unabhängige Lebensführung und Einbeziehung in die Gemeinschaft eingelöst oder seine Verwirklichung behindert wird. So gibt es keine systematischen und verlässlichen Informationen über die (regionale) Verfügbarkeit und Zugänglichkeit bedarfs- und bedürfnisgerechter, gemeindenaher Unterstützungsdienste. Auch fehlen repräsentative Daten zu Wahlmöglichkeiten bezüglich des Wohnortes und der Wohnform sowie zur subjektiven Bewertung von Möglichkeiten der Selbstbestimmung, der sozialen Einbindung und der Partizipation im Gemeinwesen seitens beeinträchtigter Menschen.

In Anbetracht dieser unzureichenden Datenlage lassen sich mögliche Missstände und Entwicklungserfordernisse gegenwärtig nur über empirische Hilfskonstruktionen ableiten. Wichtige Hinweise über Barrieren und Behinderungen der selbstbestimmten Le-

25 Hier zeigt sich die Notwendigkeit, in Forschung und Theoriebildung tradierte Differenzkategorien und ihre (Wechsel-)Wirkungen zu hinterfragen, vgl. Wansing/Westphal, Teilhabeforschung, Disability Studies und Migrationsforschung verbinden. Konzepte von Behinderung und Migration in der Forschung, Orientierung 2012, S. 12-15.

26 Vgl. hierzu auch Banafsche (Fußn. 1), S. 152 f.

27 Banafsche (Fußn. 1), S. 151 f. 
bensführung und der Einbeziehung in die Gemeinschaft liefern verfügbare Strukturdaten zur Inanspruchnahme und zu Angebotsformen wohnbezogener Hilfen ebenso wie vorliegende einzelne (regionale und qualitative) Studien zur Bewertung der Wohnsituation durch Menschen mit Beeinträchtigungen. Insgesamt können Wohninstitutionen als „zeitgebundene und insofern kulturell codierte Repräsentationen eines wandelbaren Begriffs von Behinderung" 28 als Seismograph für den Stand der Umsetzung der BRK (im Ausschnitt von Artikel 19) betrachtet werden.

\section{$V$. Strukturelle Entwicklungen der wohnbezogenen Unterstützung}

Derzeit gibt es bundesweit insgesamt etwa 340.000 Menschen mit einer amtlich anerkannten Behinderung, die wohnbezogene Leistungen der Eingliederungshilfe ${ }^{29}$ in Anspruch nehmen ${ }^{30}$. Dabei zeigt sich in den letzten zehn Jahren eine ungebrochen steigende Nachfrage ${ }^{31}$, deren Gründe vielfältig und regional unterschiedlich sind. Eine wichtige Rolle spielen eine höhere Lebenserwartung von Menschen mit (kognitiven) Beeinträchtigungen sowie eine zunehmende Nachfrage nach Unterstützung von Menschen mit psychischer Erkrankung. Obwohl es inzwischen zum Teil recht differenzierte Wohnformen für Menschen mit Beeinträchtigungen gibt, weisen die verfügbaren Statistiken keine differenzierten Angaben zur Art der in Anspruch genommenen wohnbezogenen Hilfen aus. Es wird lediglich zwischen stationär (in Wohneinrichtungen) und ambulant (eigene Wohnung oder Wohngemeinschaft) unterschieden. Dabei weisen diese Zahlen auf hohe Zuwachsraten im ambulanten Bereich bei gleichzeitig geringen Steigerungszahlen bei den stationären Leistungsempfängern hin. Gleichwohl zeigt sich, dass der Grundsatz ambulant vor stationär (gemäß § 13 SGB XII, Abs. 1) noch nicht konsequent umgesetzt wird und stationäre Wohnformen nach wie vor dominieren. Im bundesweiten Durchschnitt zeigt sich für das Jahr 2010 ein Verhältnis stationär zu ambulant von knapp zwei Drittel zu einem Drittel ${ }^{32}$. Dabei sind die Chancen der Wahlfreiheit regional höchst ungleich verteilt: In einigen Bundesländern ist die Ambulantisierung der wohnbezogenen Hilfen und der Ausbau differenzierter Unterstützungsangebote bereits deutlich vorangeschritten (z.B. Hamburg, NRW). In anderen Ländern (z.B. SachsenAnhalt, Bayern) hingegen liegt die sog. Ambulantisierungsquote deutlich unter dem

28 Rudloff, Das Ende der Anstalt?, in: Bösl/Klein/Waldschmidt (Hrsg.), Disability History. Konstruktionen von Behinderung in der Geschichte. Eine Einführung, Bielefeld 2010, S. 186.

29 Hilfen zu einem selbstbestimmten Lebens in betreuten Wohnmöglichkeiten nach § 53 SGB XII.

30 Statistisches Bundesamt, Ergebnisse der Sozialhilfestatistik 2010, Wiesbaden 2012, www.destatis. de/DE/Publikationen/WirtschaftStatistik/Sozialleistungen/ErgebnisseSozialhilfe2010_032012.pdf? blob=publicationFile.

31 Vgl. Sozialhilfestatistiken des Statistischen Bundesamtes.

32 Statistisches Bundesamt (Fußn. 30). 
Bundesdurchschnitt ${ }^{33}$ und es gibt zum Teil erhebliche Angebotslücken; dies gilt vor allem in ländlichen Regionen. Zudem sind Wahlmöglichkeiten ungleich verteilt in Abhängigkeit von der Art der Beeinträchtigung bzw. von Art und Umfang der erforderlichen Unterstützung. So sind es insbesondere Menschen mit psychischen Beeinträchtigungen, die zahlenmäßig am deutlichsten von den neuen, offenen Hilfen ${ }^{34}$ profitieren, während es für Menschen (mit kognitiven und mehrfachen Beeinträchtigungen), die auf umfassende, psychosoziale Unterstützung und Pflege bei der Alltagsbewältigung angewiesen sind, nach wie vor wenig Alternativen gibt zwischen einem Leben in einem Wohnheim oder dem Verbleib in der Herkunftsfamilie. Auf der Basis regionaler Studien und Expertenschätzungen kann man davon ausgehen, dass etwa die Hälfte der Menschen mit kognitiven Beeinträchtigungen auch noch im fortgeschrittenen Erwachsenenalter in der Herkunftsfamilie lebt 35 . Die erforderliche Unterstützung und Pflege wird in diesem Rahmen häufig ausschließlich durch Angehörige und ohne Inanspruchnahme professioneller Dienste erbracht. Auch wenn diese enorme Leistungsbereitschaft der Familien Anerkennung verdient, so wäre doch auch zu hinterfragen, inwieweit sich ein langer Verbleib in der Familie nicht im Einzelfall kontraproduktiv zur Perspektive einer selbstbestimmten Lebensführung und der Einbeziehung in das Gemeinwesen auswirken könnte. In jedem Fall gilt es aber, die Situation der Familien in sozialplanerischen Überlegungen zur Unterstützung im Gemeinwesen künftig stärker zu berücksichtigen.

Die uneingeschränkte Verwirklichung der Option, entscheiden zu können, wo und mit wem man leben möchte, und nicht verpflichtet zu sein, in besonderen Wohnformen zu leben (Art. 19, a BRK), wird für Menschen mit Beeinträchtigungen gegenwärtig strukturell durch fehlende Angebote sowie durch den Mehrkostenvorbehalt in den rechtlichen Bestimmungen der Eingliederungshilfe verhindert. Demnach gilt der gesetzliche Vorrang ambulanter Leistung nicht, „,wenn eine Leistung für eine geeignete stationäre Einrichtung zumutbar und eine ambulante Leistung mit unverhältnismäßigen Mehrkosten verbunden ist" ( 13 SGB XII). Diese Regelung schwächt faktisch die Wirksamkeit des allgemeinen Wunsch- und Wahlrechtes im SGB IX und widerspricht dem Menschenrecht auf freie Wahl der Wohnform. „Im Lichte von Art. 19 des Übereinkommens der Vereinten Nationen (...) kann eine unfreiwillige Heimunterbringung grundsätzlich nicht mehr als zumutbar gelten“36. Insbesondere für Menschen mit umfänglichen und damit kostenintensiven Unterstützungsbedarfen bedeutet der Kostenvorbehalt eine er-

33 BAGüS, con_sens, Kennzahlenvergleich der überörtlichen Träger der Sozialhilfe 2010, Hamburg 2011, http://www.consens-info.de/upload/files/projekte/z_projekte_dateien_117219_ueoeTr Benchmarking_Bericht_2010_Endfassung.pdf.

34 Gemeint ist die Gesamtheit der ambulanten, personenbezogenen sozialen Dienstleistungen, einschließlich der Persönlichen Assistenz.

35 Für die Region Westfalen-Lippe vgl. Schäfers/Wansing, Familienunterstützende Hilfen (FUH). Alternativen zum betreuten Wohnen für Menschen mit Behinderung, Münster 2009, www.lwl.org/ spur-download/fuh/fuh-bericht.pdf.

36 Welti, Kommentar zu $\$ 9$ SGB IX, in: Lachwitz/Schellhorn/Welti (Hrsg.), HK-SGB IX, 3. Aufl. Köln 2010, S. 107. 
hebliche Einschränkung ihres Selbstbestimmungsrechtes. Ein Leben in selbstgewählten Wohnformen am selbstgewählten Wohnort bleibt im Kontext dieser Vorschrift eine exklusive Möglichkeit, die ausschließlich einem unter ökonomischen Gesichtspunkten ausgewählten Personenkreis gewährt wird. Solange am grundsätzlichen Fortbestand der Leistungskategorien von stationär und ambulant festgehalten wird, solange bleibt auch der Entscheidungshorizont offen, entlang eines den Personen zugeschriebenen „stationären Hilfebedarfs" darüber zu entscheiden, welche (begrenzten) Wohnoptionen prinzipiell offen stehen. Dies stabilisiert und fördert möglicherweise Risiken der Diskriminierung und Separation von Personen mit hohen Unterstützungsbedarfen unter den finanziellen Vorzeichen der steigendenden Sozialhilfeausgaben in den nächsten Jahren.

Ebenfalls als widersprüchlich zu Artikel 19 erscheinen gelegentliche Praxen

- der „Fehlplatzierung“ junger Menschen mit Beeinträchtigungen in Alten- und Pflegeheimen ${ }^{37}$,

- des unfreiwilligen Auszugs alt werdender Menschen mit Beeinträchtigungen aus ihrer vertrauten Wohnumgebung (in Einrichtungen der Behindertenhilfe) in Alten- und Pflegeheime sowie

- der unfreiwilligen Auswärtsunterbringung von Bürgerinnen und Bürger eines Herkunftsgebietes in Einrichtungen anderer Regionen.

\section{Bewertungen und Wünsche aus Nutzerperspektive}

Eine qualitative Bewertung der Wohnsituation und hierauf bezogener Wahlfreiheiten ist im Lichte der Grundsätze von Selbstbestimmung und Partizipation letztlich nur aus der Perspektive der betroffenen Menschen möglich. Wie bewerten Menschen mit alltäglichen Unterstützungsbedarfen selbst ihre gegenwärtige Wohnsituation? Welche Wünsche und Bedürfnisse haben sie in Bezug auf ihre alltägliche Lebensführung, welche Unterstützung wünschen sie, und wie würden sie entscheiden, wenn sie reale Chancen hätten, ihren Aufenthaltsort zu wählen und zu entscheiden, wo und mit wem sie leben? Im Zuge der wachsenden Bedeutung partizipativer Forschung liegen inzwischen verschiedene Studien vor, welche die subjektiven Wahrnehmungen und Bewertungen der beeinträchtigten Menschen in den Vordergrund stellen. Diese zeigen - trotz unterschiedlicher Wohnformen, regionaler Kontexte und Altersgruppen der Befragten - hohe Übereinstimmungen zu zentralen Aspekten der wohnbezogenen Unterstützung. Im Folgenden sollen einige ausgewählte, im Kontext von Artikel 19 bedeutsame Ergebnisse skizziert werden. Dabei beziehe ich mich im Wesentlichen auf folgende drei Studien:

37 Drolshagen, „Was mir fehlt, ist ein Zuhause“. Fehlplatzierung jüngerer Behinderter in hessischen Altenhilfe-Einrichtungen, Berlin 2006. 


\begin{tabular}{|c|c|c|c|c|}
\hline & Erhebungsregion & Wohnformen & $\begin{array}{l}\text { Erhebungs- } \\
\text { methode }\end{array}$ & $\begin{array}{l}\text { Anzahl der } \\
\text { Befragten }\end{array}$ \\
\hline „Kundenstudie“‘38 & $\begin{array}{l}\text { Berlin (ausgewählte } \\
\text { Bezirke) }\end{array}$ & $\begin{array}{l}\text { Familie, ambulant betreutes } \\
\text { Wohnen, Wohnheime }\end{array}$ & Fragebögen & $\mathrm{N}=207$ \\
\hline $\begin{array}{l}\text { „Lebensqualität } \\
\text { aus Nutzersicht“‘39 }\end{array}$ & $\begin{array}{l}\text { NRW (Bielefeld), } \\
\text { Baden-Württemberg } \\
\text { (Nordbaden) }\end{array}$ & Wohnheime & Interviews & $\mathrm{N}=142$ \\
\hline „Wohnen inklusiv“40 & Baden-Württemberg & $\begin{array}{l}\text { Familie, Wohngruppe, } \\
\text { Wohnheim }\end{array}$ & Fragebögen & $\mathrm{N}=931$ \\
\hline
\end{tabular}

Zunächst fällt auf, dass sich etwa die Hälfte der befragten Menschen allgemein zufrieden mit der gegenwärtigen Wohnsituation äußert, und zwar unabhängig von der jeweiligen Wohnform. Dieser Befund lässt verschiedene Interpretationen zu:

- Die Wohnsituation entspricht tatsächlich den individuellen Bedürfnissen und Vorstellungen;

- es sind keine Alternativen bekannt. Verschiedene Befragungsergebnisse weisen darauf hin, dass viele Menschen mit Beeinträchtigungen und ihre Angehörigen nicht gut über alternative Wohnmöglichkeiten oder das persönliche Budget informiert sind;

- die grundsätzliche Zufriedenheit lässt sich aber auch mit dem aus der Forschung bekannten Zufriedenheitsparadoxon bzw. mit der „Theorie der Homöostase subjektiven Wohlbefindens“" des australischen Lebensqualitätsforschers Cummins erklären ${ }^{41}$. Demzufolge sind die meisten Menschen bestrebt, ein gewisses Niveau an Wohlbefinden unter variierenden (auch ungünstigen) Lebensbedingungen aufrecht zu erhalten und erreichen dies ggf. auch auf dem Wege resignativer Anpassung. Für diese Erklärung sprechen einzelne Äußerungen von befragten Menschen wie ,am besten man lässt alles so wie es ist, sonst eckst du nur an“ oder „was soll ich schon ändern“42.

Trotz der allgemeinen Zufriedenheitswerte gibt jedoch mindestens jede zweite befragte Person Veränderungswünsche an mit der Perspektive, zukünftig anders wohnen zu wollen. Dies kann durchaus als Ausdruck dafür gedeutet werden, dass das Leben in den jeweiligen Wohnformen nicht den Bedürfnissen und Lebenszielen dieser Personen

38 Seifert, Kundenstudie, Bedarf an Dienstleistungen zur Unterstützung des Wohnens von Menschen mit Behinderung, Berlin 2010.

39 Schäfers, Lebensqualität aus Nutzersicht. Wie Menschen mit geistiger Behinderung ihre Lebenssituation beurteilen, Wiesbaden 2008.

40 Metzler/Rauscher, Wohnen inklusiv. Wohn- und Unterstützungsangebote für Menschen mit Behinderungen in Zukunft (Projektbericht), Stuttgart 2004.

41 Schäfers (Fußn. 39), S. 46.

42 Schäfers (Fußn. 39), S. 256. 
entspricht. Hinweise hierauf liefern teils drastische Äußerungen von Wohnheimbewohner/innen wie „,ich würde die Einrichtung abreißen“, „Anstalt schließen“ oder „dass ich hier rauskomme" 43 . Dabei zeigen einige Formulierungen der Befragten, „dass sie die Wohneinrichtungen als geschlossen und vom Gemeindeleben getrennten Lebensraum erleben, z.B. wenn sie Wünsche danach äußern „draußen leben“ oder ,außerhalb wohnen“ zu können“44. Die konkrete Kritik dieser Personen in betreuten Wohngemeinschaften oder Wohngruppen eines Heims richtet sich auch auf das Zusammenleben mit Menschen, die in der Regel nicht selbst ausgesucht wurden. Zwar wird das Leben in Gemeinschaft von einigen Personen durchaus geschätzt, allerdings wird die Frage ,gibt es Menschen in der Wohngruppe/Wohnung, mit denen Sie nicht gern zusammen wohnen?" von der Hälfte der befragten Personen in der Studie von Schäfers bejaht ${ }^{45}$. Auch in der Kundenstudie von Seifert steht das häufig konfliktreiche Zusammenleben in Gruppen bei den negativen Aspekten des Alltags an erster Stelle ${ }^{46}$. Diese Ergebnisse legen erhebliche Diskrepanzen offen zwischen der faktischen Wohnsituation beeinträchtigter Menschen und ihrem Recht, darüber zu entscheiden mit wem sie leben möchten (Art. 19 a) BRK).

Ein autonomes Leben nach eigenen Vorstellungen zu führen, bedeutet auch, den Alltag nach eigenem zeitlichem Rhythmus gestalten zu können. Diesbezüglich wünschen sich Wohnheimbewohner weniger Reglementierungen und mehr Handlungsspielräume; dies betrifft z.B. die Abschaffung von Badeplänen, abendlichen Rückkehrzeiten und die Möglichkeit, Freunde und Partner/innen abends besuchen zu dürfen ${ }^{47}$. Diese konkreten Beispiele zeigen, wie begrenzt Entscheidungsspielräume für die Gestaltung des Alltags in Einrichtungen im Vergleich zu kulturell üblichen Spielräumen nach wie vor sein können. Dabei scheint sich dass das Ausmaß an erlebter Selbstbestimmung und Wahlfreiheit in ambulanten Wohnformen deutlich von stationären Wohneinrichtungen zu unterscheiden und zugleich mit der Größe von Wohneinheiten zusammen zu hängen. So gaben in der Studie von Seifert fast alle befragten Personen, die in einer eigenen Wohnung leben, an, selbst über die Gestaltung des Alltags bestimmen zu können. Hingegen stimmten nur etwa die Hälfte der Wohnheimbewohner/innen dieser Aussage zu ${ }^{48}$. Auch bei Schäfers sehen fast die Hälfte der befragten Wohnheimbewohner/innen eher wenige Wahlfreiheiten (z.B. bei der Entscheidung darüber, welche Person zu welchem Zeitpunkt Hilfen erbringen); mit steigendem Hilfebedarf nehmen die erlebten Wahlfreiheiten ab. Zugleich äußern Personen in Wohnheimen ohne Wohngruppenbezug bzw. in

43 Schäfers (Fußn. 39), S. 330.

44 Schäfers (Fußn. 39), S. 330.

45 Schäfers (Fußn. 39), S. 254.

46 Seifert (Fußn. 38), S. 104.

47 Schäfers (Fußn. 39), S. 257.

48 Seifert (Fußn. 38), S. 106. 
sehr kleinen Gruppen (bis zu 3 Personen) deutlich höhere Zufriedenheitswerte mit Wahlmöglichkeiten als in größeren Wohngruppen ${ }^{49}$.

Wenn man vor diesem Hintergrund davon ausgeht, dass die Entscheidungsspielräume und Handlungsoptionen in ambulanten Wohnformen grundsätzlich größer sind, dann überrascht zunächst ein Ergebnis der Kundenstudie, demnach sich nur knapp die Hälfte der Menschen im betreuten Einzelwohnen zufrieden mit ihrer Wohnsituation äußert ${ }^{50}$. Die Kritik der Unzufriedenen bezieht sich jedoch nicht auf die Wohnform an sich, sondern auf die konkreten Wohnbedingungen, wie die Beschaffenheit der Wohnung und das (soziale) Wohnumfeld (z.B. kleine Wohnung, schlechte Infrastruktur, laute Nachbarn). Menschen, die alleine in einer Wohnung leben, äußern darüber hinaus zum Teil Einsamkeitsgefühle oder Probleme mit ihrem Partner. Im Bereich der sozialen Einbindung bestehen insgesamt Defizite in allen Wohnformen, dies bestätigen auch andere vorliegende Studien ${ }^{51}$. Vor allem Menschen mit kognitiven und psychischen Beeinträchtigungen in Wohneinrichtungen haben deutlich eingeschränkte informelle soziale Kontakte, insbesondere zu Personen außerhalb der Einrichtungen, und zugleich ein hohes Maß an nicht selbstgewählten Beziehungen, etwa zu Mitarbeiter/innen in den Einrichtungen und Diensten.

Gefragt nach ihren Wohnwünschen äußern die Personen insgesamt eine Vielfalt an Vorstellungen, wie und mit wem sie gerne wohnen und leben möchten: alleine, in Partnerschaft oder in einer Wohngemeinschaft mit Freunden, in der Familie usw. Diese Vielfalt der Wohnwünsche spiegelt allgemeine gesellschaftliche Tendenzen hin zu pluralen Wohn- und Lebensformen. Und die Ergebnisse unterstreichen die Unterschiedlichkeit von Menschen mit Beeinträchtigungen sowie die Notwendigkeit, von pauschalen Vorstellungen „behindertengerechter“ Wohnformen abzurücken. Am häufigsten wird in allen Studien das eigenständige Wohnen, alleine oder mit Partner/in, genannt. Hingegen spielen Wohnheime, Wohngemeinschaften und das Zusammenleben mit Angehörigen bei den Wohnwünschen der Personen mit Veränderungswünschen zahlenmäBig nur eine sehr nachrangige Rolle.

\section{Fazit und Perspektiven der Enthinderung}

Die Darstellung und Diskussion von Ergebnissen zur Wohnsituation von Menschen mit Beeinträchtigungen kann im Rahmen dieses Beitrages nur holzschnittartig bleiben. Insbesondere bleiben Kontextfaktoren im Sozialraum aufgrund der unzureichenden Da-

49 Schäfers (Fußn. 39), S. 332.

50 Seifert (Fußn. 38), S. 101.

51 Heckmann, Alltags- und Belastungsbewältigung und soziales Netzwerk, in: Beck/Greving (Hrsg.), Lebenslage, Lebensbewältigung. Enzyklopädisches Handbuch der Behindertenpädagogik Band 5, Stuttgart 2012, S. 115-123. 
tenlage ausgeblendet. Gleichwohl lassen bereits diese Ergebnisse im Zusammenhang mit den zuvor skizzierten Strukturdaten erhebliche Diskrepanzen zwischen den in Artikel 19 formulierten Ansprüchen auf eine selbstbestimmte Lebensführung und den faktischen Wahlmöglichkeiten im Kontext der organisierten wohnbezogenen Unterstützung erkennen. Die Vielfalt der Wohnwünsche mit einem hohen Stellenwert „einer eigenen Wohnung mit der individuell notwendigen Assistenz, in sozialen Bezügen und einem

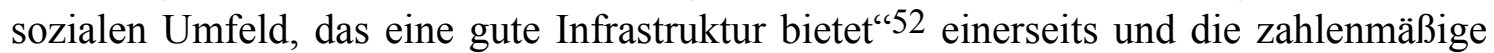
Dominanz stationärer Einrichtungen, die finanziellen sowie rechtlich-administrativen Hürden bei der Umsetzung individuell passender Hilfen, einschließlich integrierter, trägerübergreifender Lösungen (beispielsweise im Verhältnis von Pflege und Eingliederungshilfe) andererseits klaffen weit auseinander. Das Recht zu entscheiden, wo und mit wem man leben möchte, lässt sich in diesem Rahmen ebenso unzureichend verwirklichen wie die Gestaltung eines Alltags nach eigenen Vorstellungen.

Vor diesem Hintergrund vollziehen sich bereits seit einigen Jahren wesentliche Schritte der Neuausrichtung von Wohnkonzepten für beeinträchtigte Menschen in Richtung personenzentrierter und gemeindeorientierter Unterstützung. Insbesondere mögliche Anpassungen der Eingliederungshilfe an politische, rechtliche, fachliche und gesamtgesellschaftliche Entwicklungen stehen gegenwärtig stark im Fokus ${ }^{53}$. Eine zentrale Rolle spielt im Rahmen dieser Bemühungen der konsequente Ausbau offener Hilfen. Allerdings zeigen die Ergebnisse zur Bewertung der Situation in ambulant betreuten Wohnformen, dass eine Umsteuerung von stationären zu ambulanten Hilfen allein nicht mehr Wohn- und Lebensqualität garantieren kann. Zwar ermöglicht das Leben in einer eigenen Wohnung strukturell ein hohes Maß an Selbstbestimmung und Wahlfreiheiten bei der Gestaltung des Alltags, es verhindert jedoch nicht per se Risiken sozialer Diskriminierung und der „Isolation und Absonderung von der Gemeinschaft“ (Art. 19 c BRK). Es bedarf vielmehr der individuellen Unterstützung bei der aktiven Herstellung und Aufrechterhaltung von Kontakten und Beziehungen im sozialen Nahraum, um eine Einbindung auch auf der Ebene sozialer Interaktion zu ermöglichen. Dies gilt umso mehr, als Menschen mit Beeinträchtigungen ohne Möglichkeiten informeller Unterstützung vollständig von professionellen, offenen Hilfen abhängig sind, die aber gegenwärtig in vielen Regionen eben nicht bedarfsgerecht verfügbar sind.

Ein zentraler Schlüssel für eine zielführende Neuausrichtung der sozialen Hilfen im Lichte von Artikel 19 BRK liegt in der Überwindung der Leistungskategorien ambulant und stationär und einer konsequenten Umstellung von einer Kategorie des „Wohnens“ auf Kategorien der Lebensführung bzw. der Lebens- und Alltagsbewältigung. „Wohnen" steht im System der traditionellen Behindertenhilfe für eine etablierte institutionelle Kategorie, die auf der rechtlich-administrativen Konstruktion eines pauschalen Hilfe-

52 Seifert (Fußn. 38), S. 21 f.

53 Vgl. Beschluss der 86. ASMK-Konferenz 2009 zur Weiterentwicklung der Eingliederungshilfe für Menschen mit Behinderungen (,Eckpunkte für die Reformgesetzgebung Eingliederungshilfe im SGB XII“). 
bedarfs gründet, an den sich in der Regel, auf der Basis pauschaler Leistungs- und Vergütungsvereinbarungen zwischen Sozialhilfeträger und Anbieter, eine professionell organisierte Wohnform anschließt. Eine wirksame Unterstützung ist jedoch keine Frage nach dem richtigen Gebäude, oder anders formuliert: Das Wohnen ist kein Unterstützungsbedarf. Jede zielführende Intervention zur Teilhabe am Leben in der Gemeinschaft, die der Pluralisierung von Wohn- und Lebensformen und dem Postulat der Selbstbestimmung Rechnung tragen will, muss sich an der individuellen Lebensführung im Sinne des alltagspraktischen Zusammenhangs aller Aktivitäten einer Person in ihren verschiedenen konkreten Lebensbereichen ausrichten ${ }^{54}$. In diesem Betrachtungsrahmen gilt es danach zu fragen, wie Barrieren im Zugang zu sozial anerkannten Verwirklichungsräumen abgebaut, wie funktionale Beeinträchtigungen bewältigt und mit welchen Ressourcen der Alltag nach eigenen Vorstellungen gelingen kann. Diese Aufgaben lassen sich nicht mit pauschalen Maßnahmen im Sinne „behindertengerechter" Wohnformen lösen, sondern sind höchst individuell in Abhängigkeit von persönlichen Voraussetzungen (Ressourcen, Fähigkeiten und Bedarfe/Bedürfnisse) und von konkreten regionalen und sozialen Bezügen am selbstgewählten Wohn- und Lebensort zu bearbeiten. Erforderlich ist daher eine enge Verknüpfung von Sozialraumentwicklung und der Weiterentwicklung personenzentrierter Hilfen bzw. ein optimiertes Zusammenspiel von örtlicher und regionaler Teilhabeplanung, die auf die Lebenslagen beeinträchtigter Menschen gerichtet ist ${ }^{55}$, mit Ansätzen individueller Hilfeplanung.

54 Zum soziologischen Konzept der alltäglichen Lebensführung vgl. Kudera/Voß (Hrsg.), Lebensführung und Gesellschaft, Opladen 2000.

55 Blankenfeld/Wachtel, Auf dem Weg ins Gemeinwesen - kommunale Praxis für Menschen mit Behinderung in Theorie und Praxis, VHN 2009, S. 197-211; Lampke/Rohrmann/Schädler, Örtliche Teilhabeplanung mit und für Menschen mit Behinderungen, Wiesbaden 2011. 


\section{Literatur}

Banafsche, Minou, Art. 19 Unabhängige Lebensführung und Einbeziehung in die Gemeinschaft, in: Welke, Antje (Hrsg.), UN-Behindertenrechtskonvention mit rechtlichen Erläuterungen. Deutscher Verein, Berlin 2012, S. 150-163.

BAGüS/con_sens, Kennzahlenvergleich der überörtlichen Träger der Sozialhilfe 2010, Hamburg 2011, http://www.consens-info.de/upload/files/projekte/z_projekte_dateien_ 117219_ueoeTr_Benchmarking_Bericht_2010_Endfassung.pdf.

Bank-Mikkelsen, Niels, Denmark, in: Flynn, Robert/Nitsch, Cathreen (Hrsg.), Normalization, social integration, and community services, Baltimore 1980, S. 51-70.

Beck, Iris/Greving, Heinrich (Hrsg.), Lebenslage und Lebensbewältigung. Enzyklopädisches Handbuch der Behindertenpädagogik 5, Stuttgart 2012.

Beck, Iris/Greving, Heinrich, Lebenswelt, Lebenslage, in: dies. (Hrsg.), Lebenslage und Lebensbewältigung. Enzyklopädisches Handbuch der Behindertenpädagogik 5, Stuttgart 2012, S. 15-59.

Bielefeldt, Heiner, Zum Innovationspotenzial der UN-Behindertenrechtskonvention. Deutsches Institut für Menschenrechte. 3. Aufl. Berlin 2009.

Blankenfeld, Christine/Wachtel, Grit, Auf dem Weg ins Gemeinwesen - kommunale Praxis für Menschen mit Behinderung in Theorie und Praxis, VHN 2009, S. 197-211.

Burzan, Nicole et al., Das Publikum der Gesellschaft. Inklusionsverhältnisse und Inklusionsprofile in Deutschland, Wiesbaden 2008.

Drolshagen, Markus, „Was mir fehlt, ist ein Zuhause“. Fehlplatzierung jüngerer Behinderter in hessischen Altenhilfe-Einrichtungen, Berlin 2006.

Engels, Dietrich, Lebenslagen, in: Maelicke, Bernd (Hrsg.), Lexikon der Sozialwirtschaft, Baden-Baden 2008, S. 643-646.

Hahn, Martin, Behinderung als soziale Abhängigkeit. Zur Situation schwerbehinderter Menschen, München 1981.

Heckmann, Christoph, Alltags- und Belastungsbewältigung und soziales Netzwerk, in: Beck, Iris/Greving, Heinrich (Hrsg.), Lebenslage, Lebensbewältigung. Enzyklopädisches Handbuch der Behindertenpädagogik. Band 5, Stuttgart 2012, S. 115-123.

Hornberg, Claudia/Schröttle, Monika, Vorstudie zur Neukonzeption des Behindertenberichts, Bundesministerium für Arbeit und Soziales (Hrsg.), Forschungsbericht 408, 2011, http:/www.bmas.de/DE/Service/Publikationen/Forschungsberichte/ForschungsberichteTeilhabe/fb-fb408-vorstudie-zur-neukonzeption-des-behindertenberichtes.html.

Kudera, Werner/Voß, Günther (Hrsg.), Lebensführung und Gesellschaft, Opladen 2000.

Lampke, Dorothea/Rohrmann, Albrecht/Schädler, Johannes, Örtliche Teilhabeplanung mit und für Menschen mit Behinderungen, Wiesbaden 2011.

Leßmann, Ortrud, Lebenslagen und Verwirklichungschancen (capability) - Verschiedene Wurzeln, ähnliche Konzepte, Vierteljahreshefte zur Wirtschaftsforschung 75/2006, 1, S. 30-42.

Luhmann, Niklas, Die Gesellschaft der Gesellschaft. Bd. 2, Frankfurt a.M. 1999. 
Rappaport, Julian, Ein Plädoyer für die Widersprüchlichkeit: Ein sozialpolitisches Konzept des „empowerment“" anstelle präventiver Ansätze, Verhaltenstherapie und psychosoziale Praxis $2 / 1985$, S. 257-278.

Rudloff, Wilfried, Das Ende der Anstalt?, in: Bösl, Elsbeth/Klein, Anne/Waldschmidt, Anne (Hrsg.), Disability History. Konstruktionen von Behinderung in der Geschichte. Eine Einführung, Bielefeld 2010, S. 169-190.

Schäfers, Markus, Lebensqualität aus Nutzersicht. Wie Menschen mit geistiger Behinderung ihre Lebenssituation beurteilen, Wiesbaden 2008.

Schäfers, Markus/Wansing, Gudrun, Familienunterstützende Hilfen (FUH). Alternativen zum betreuten Wohnen für Menschen mit Behinderung, Münster 2009, http://www.lwl.org/ spur-download/fuh/fuh-bericht.pdf.

Schlebrowski, Dorothee, Starke Nutzer im Heim. Wirkung Persönlicher Budgets auf soziale Dienstleistungen, Wiesbaden 2009.

Seifert, Monika, Kundenstudie. Bedarf an Dienstleistungen zur Unterstützung des Wohnens von Menschen mit Behinderung, Berlin 2010.

Sen, Amartya, Ökonomie für den Menschen. Wege zur Gerechtigkeit und Solidarität in der Marktwirtschaft, München, Wien 2000.

Statistisches Bundesamt, Ergebnisse der Sozialhilfestatistik 2010, Wiesbaden 2012. Download unter: https://www.destatis.de/DE/Publikationen/WirtschaftStatistik/Sozialleistungen/ ErgebnisseSozialhilfe2010_032012.pdf?_blob=publicationFile.

Thimm, Walter, Leben in Nachbarschaften. Hilfen für Menschen mit Behinderungen, Freiburg 1994.

Thimm, Walter, Tendenzen gemeinwesenorientierter Hilfen - Gesellschaftliche Ausrichtungen und fachliche Konsequenzen, in: ders. (Hrsg.), Das Normalisierungsprinzip. Ein Lesebuch zu Geschichte und Gegenwart eines Reformkonzeptes, Marburg 2005.

Waldschmidt, Anne, Selbstbestimmung als Konstruktion. Alltagstheorien behinderter Frauen und Männer, 2. korr. Auflage Wiesbaden 2012.

Wansing, Gudrun, Der Inklusionsbegriff in der Behindertenrechtskonvention, in: Welke, Antje (Hrsg.), Behindertenrechtskonvention und rechtliche Erläuterungen, Berlin 2012, S. 93103.

Wansing, Gudrun, Inklusion in einer exklusiven Gesellschaft - oder: wie der Arbeitsmarkt Teilhabe behindert, Behindertenpädagogik 4/2012, S. 381-396.

Wansing, Gudrun/Westphal, Manuela, Teilhabeforschung, Disability Studies und Migrationsforschung verbinden. Konzepte von Behinderung und Migration in der Forschung, Orientierung 1/2012, S. 12-15.

Welti, Felix, Kommentar zu $§ 9$ SGB IX, in: Lachwitz, Klaus/Schellhorn, Walter/Welti, Felix (Hrsg.), HK-SGB IX, 3. Aufl. Köln 2010.

WHO, World Report on Disability, Genf 2011. 


\title{
Rechtliche Grundlagen einer örtlichen Teilhabeplanung ${ }^{1}$
}

\author{
Felix Welti
}

$\begin{array}{ll}\text { I. Einführung } & 87\end{array}$

II. Pflicht zur Teilhabeplanung? $\quad 89$

$\begin{array}{ll}\text { 1. Verfassungsrecht } & 90\end{array}$

2. Behindertenrechtskonvention 90

3. Bundes- und Landesgesetze 92

III. Teilhabeplanung durch Städte, Gemeinden und Kreise 93

1. Kommunale Selbstverwaltung 93

2. Kommunen als Träger staatlicher Aufgaben 99

3. Beteiligung behinderter Menschen 95

IV. Regionale Teilhabeplanung durch Sozialleistungsträger 96

1. Planungsverantwortung nach SGB I und SGB X 96

2. Planungsverantwortung für Leistungen zur Teilhabe nach SGB IX 97

3. Planungsverantwortung für Pflegeleistungen nach SGB XI 98

4. Gesundheitsplanung 99

$\begin{array}{ll}\text { V. Schluss } & 100\end{array}$

\section{Einführung}

Die Aktivitäten behinderter Menschen und ihrer Verbände für mehr Selbstbestimmung und Teilhabe, die neuere wissenschaftliche und fachliche Diskussion, insbesondere über die Internationale Klassifikation der Behinderung, Funktionsfähigkeit und Gesundheit (ICF) ${ }^{2}$, und die Gesetzgebung mit SGB IX, Behindertengleichstellungsgeset-

1 Frühere Fassungen dieses Textes sind erschienen in ZfSH/SGB 2011, S. 401-406, und in Dorothea LampkelAlbrecht Rohrmann/Johannes Schädler (Hrsg.), Örtliche Teilhabeplanung mit und für Menschen mit Behinderungen, Wiesbaden 2011, S. 55-67.

2 Zum Zusammenhang zwischen der International Classification of Functioning, Disability and Health (ICF) der WHO und dem SGB IX auch Wolfgang Seger/Hans-Martin Schian/Bernd Steinke/Wolfgang Heipertz/Michael Schuntermann, Gesundheits-, sozial-, gesellschafts- und organisationspolitische Auswirkungen der Anwendung der ICF auf eine integrierte Rehabilitation, Das Gesundheitswesen 2004, S. 393-399. 
zen mit Regelungen zur Barrierefreiheit ${ }^{3}$, Allgemeinem Gleichbehandlungsgesetz und der Behindertenrechtskonvention der Vereinten Nationen haben den Blick dafür geöffnet, dass Menschen nicht nur durch gesundheitliche Einschränkungen von Funktionsfähigkeit und Aktivitäten behindert sind, sondern auch durch gestaltbare gesellschaftliche Kontextfaktoren behindert werden.

Damit ist deutlicher geworden, dass die politische, gesellschaftliche und rechtliche Verantwortung des sozialen Rechtsstaats sich nicht nur darauf beschränken kann, externalisierende Interventionen bereitzustellen, die am Gesundheitszustand ansetzen oder die Ausgrenzung aus allgemeinen Lebenszusammenhängen durch besondere Dienste und Einrichtungen zu kompensieren. Die Gestaltung und Planung in der Gesellschaft soll vielmehr stärker darauf ausgerichtet werden, behinderte Menschen in Bildung, Arbeitsleben, Kultur, Politik und Gesellschaft einzubeziehen. Die sozialstaatliche Integration von Staat und Gesellschaft soll damit durch individuelle Rechte auf Teilhabe und Inklusion ergänzt und erneuert werden ${ }^{4}$.

Schon die traditionellen Unterstützungsleistungen bei Behinderung durch Träger der Bildung, der Kinder- und Jugendhilfe, der Arbeitsmarktpolitik, der Rehabilitation, Krankenbehandlung und Pflege wurden und werden vielfach in ihrer individuellen und gesellschaftlichen Wirksamkeit durch mangelnde Koordination und Kooperation eingeschränkt. Die komplexen Zuständigkeiten staatlicher und gesellschaftlicher Ebenen von Gesetzgebung und Verwaltung sowie von professionellen und wissenschaftlichen Systemen, etwa der Pädagogik, Medizin und Pflege, stehen einer ,ganzheitlichen“ Hilfe entgegen 5 . In der modernen Lebensrealität kann ,ganzheitliche“ Hilfe nur unter den ausgrenzenden Bedingung der Anstalt und des Heims erfolgen, denn nicht ausgegrenzte behinderte Menschen sind ebenso in vielfältige, voneinander getrennte Lebenszusammenhänge einbezogen wie nicht behinderte Menschen auch. Es führt also kein vernünftiger Weg zu einer radikalen Vereinfachung von Zuständigkeiten, sondern Koordination und Planung sind ebenso bei der individuellen Unterstützung für behinderte Menschen wie bei der Gestaltung der lebensweltlichen Kontextfaktoren erforderlich. Insoweit muss die personenbezogene Ausgestaltung der Leistungen Ausgangspunkt nach geltendem Recht und in Reformbemühungen ${ }^{6}$ sein; sie ist jedoch auf sozialräumliche Voraussetzungen angewiesen.

3 Vgl. Felix Welti, Rechtliche Voraussetzungen von Barrierefreiheit in Deutschland, in: Anja Tervooren/Jürgen Weber (Hrsg.), Wege zur Kultur - Barrieren und Barrierefreiheit in Kultur- und Bildungseinrichtungen, Köln 2012, S. 67-84; aktualisiert und gekürzt in NVwZ 2012, S. 725-730.

4 Vgl. Hans F. Zacher, Einschluss, Ausschluss und Öffnung im Wandel, Zeitschrift für Sozialreform 2009, S. 25-39.

5 Vgl. Felix Welti, Systematische Stellung des SGB IX im Sozialgesetzbuch - Zusammenarbeit der Leistungsträger und Koordination der Leistungen, SGb 2008, S. 321-331.

6 Wolfgang Schütte, Abschied vom Fürsorgerecht - Rechtliche und sozialpolitische Anforderungen an eine Reform der „Eingliederungshilfe“, in: ders. (Hrsg.), Abschied vom Fürsorgerecht - Von der „Eingliederungshilfe für behinderte Menschen“ zum Recht auf soziale Teilhabe, Berlin 2011. 
Die individuelle Teilhabeplanung ist bislang Gegenstand des Sozialleistungsrechts und hier insbesondere des Rechts der Rehabilitation und Teilhabe. Die Rehabilitationsträger sind zu einer trägerübergreifenden Bedarfsfeststellung verpflichtet, deren Koordination in ihrer gemeinsamen Empfehlung „Teilhabeplan“7 geregelt ist. Alle Rehabilitationsträger, auch die örtlichen Träger der Sozialhilfe und der Kinder- und Jugendhilfe, sind verpflichtet, sich an dieser gemeinsamen Empfehlung zumindest zu orientieren ${ }^{8}$. Daneben bestehen weitere trägerspezifische Planungspflichten durch den Gesamtplan der Träger der Sozialhilfe ${ }^{9}$ und den Versorgungsplan der Pflegekasse ${ }^{10}$.

Diese individuelle Teilhabeplanung ${ }^{11}$ ist $\mathrm{zu}$ unterscheiden von einer umfassenden, auf die Verfügbarkeit von unterstützenden Leistungen und auf die Gestaltung der Kontextfaktoren im Sinne von Zugänglichkeit zielenden Teilhabeplanung auf regionaler Ebene.

Eine so verstandene örtliche Teilhabeplanung ist zunächst ein politischer und behördeninterner Prozess, dessen Ergebnis nur die Verwaltung intern bindet. Soll er Rechtsfolgen haben, die Bürgerinnen und Bürger belasten oder begünstigen, so sind diese in entsprechende Rechtsformen umzusetzen, also in den Kommunen insbesondere in Satzungen über den Haushalt oder über die Bauleitplanung, in Verwaltungsakte mit förderndem und belastendem Inhalt. Da Teilhabepläne nach jetzigem Rechtsstand keine unmittelbare Außenwirkung haben könnten, wären auch die Regelungen des Verwaltungsverfahrensrechts über Planfeststellung 12 nicht anwendbar. Gleichwohl wäre es sinnvoll, die dort enthaltenen Grundgedanken einer frühzeitigen und umfassenden Beteiligung der Betroffenen ${ }^{13}$ bei der Teilhabeplanung zu berücksichtigen.

\section{Pflicht zur Teilhabeplanung?}

Wird eine solche Teilhabeplanung für notwendig oder wünschenswert gehalten, so stellt sich die Frage, ob eine Pflicht dazu bestehen könnte.

7 Gemeinsame Empfehlung „Teilhabeplan“ v. 16. Dezember 2004 auf www.bar-frankfurt.de; $\S \S 10$ Abs. 1,13 Abs. 2 Nr. 3 SGB IX.

$8 \S 13$ Abs. 5 S. 2 SGB IX.

$9 \S 58$ SGB XII.

$10 \S 7$ a Abs. 1 S. 2 Nr. 2 SGB XI.

11 Vgl. Wolfgang Seger, Die prozessualen und strukturellen Schritte zur erfolgreichen trägerübergreifenden gemeinsamen Teilhabeplanung, Sozialer Fortschritt 2012, S. 127-132; Felix Welti, Rechtsfragen der Bedarfsfeststellung für Leistungen zur Teilhabe, Die Rehabilitation 2011, S. 7-10; ErnstWilhelm Luthe, Die Koordinationsanforderungen des SGB IX bei der Feststellung des Einzelfallbedarfs, Behindertenrecht 2010, S. 57-66; Felix Welti, Planung individueller Teilhabeleistungen durch Rehabilitationsträger, Recht der sozialen Dienste und Einrichtungen 2006 (60), S. 50-69.

$12 \S \S 72-78 \mathrm{VwVfG}$ und entsprechendes Landesrecht.

$13 \S 73 \mathrm{VwVfG}$. 


\section{Verfassungsrecht}

Nach Art. 20 Abs. 1 GG ist die Bundesrepublik Deutschland ein sozialer Rechtsstaat, nach Art. 28 Abs. 1 GG müssen es auch ihre Länder sein. Ein sozialer Staat gestaltet die Gesellschaft und wertet dabei die in ihr vorhandenen Interessen gleichmäßig. Er darf behinderte Menschen nicht vernachlässigen, sondern muss ihnen jedenfalls ein Minimum an persönlicher Entfaltung garantieren.

Das Benachteiligungsverbot wegen einer Behinderung (Art. 3 Abs. 3 Satz 2 GG) kann nicht nur als individuelles Gebot der Rechtsgleichheit verstanden werden ${ }^{14}$, sondern ist auch ein Gebot an den Staat, gleiche Chancen und Entfaltungsmöglichkeiten durch angemessene Vorkehrungen zu schaffen ${ }^{15}$. Acht deutsche Länder, darunter der Freistaat Bayern, verdeutlichen den sozialen Staatsauftrag für behinderte Menschen in ihren Verfassungen ausdrücklich ${ }^{16}$.

Aus diesen verfassungsrechtlichen Vorgaben lässt sich ablesen, dass die Verantwortung für behinderte Menschen bei allen staatlichen Ebenen liegt. Sie kann nicht, etwa durch eine spezifische Gesetzgebungs- oder Verwaltungskompetenz, Bund, Ländern, Gemeinden oder Sozialversicherungsträgern exklusiv zugeordnet werden. Diese nach dem Recht und in der Sache richtige Teilung der Verantwortung führt dazu, dass für eine Teilhabeplanung keine eindeutige Verantwortlichkeit oder Federführung abgeleitet werden kann. Vielmehr sind zunächst alle Träger der unmittelbaren und mittelbaren Staatsverwaltung jeweils im Rahmen ihrer Zuständigkeiten verpflichtet, die auf Einbeziehung behinderter Menschen gerichteten Staatsziele zu beachten. Daraus lässt sich keine konkretisierte Planungspflicht ableiten, wohl aber eine Pflicht jeweils darauf zu achten, wie sich das eigene Handeln auch außerhalb spezifisch behinderungsbezogener Aktivität auf behinderte Menschen auswirkt. Insoweit ist eine Vernetzung von Informationen und Entscheidungen jedenfalls zweckmäßig.

\section{Behindertenrechtskonvention}

Die Behindertenrechtskonvention der Vereinten Nationen (BRK) gilt seit dem 26. März 2009 als einfaches Bundesrecht ${ }^{17}$. Sie bindet auch die Länder im Bereich ihrer Kompetenzen ${ }^{18}$. Die BRK kann, wie andere Menschenrechtsübereinkommen ${ }^{19}$, auch

14 Dazu BVerfG v. 19. Januar 1999, BVerfGE 99, 341.

15 BVerfG v. 8. Oktober 1997, BVerfGE 96, 288, 303; Art. 5 Abs. 3 BRK; Art. 5 RL 2000/78 EG.

16 Art. 118a S. 2 BayVerf; Art. 11 S. 2 BlnVerf; Art. 45 Abs. 3 BbgVerf; Art. 17a MVVerf; Art. 64 RhPfVerf; Art. 7 Abs. 2 SächsVerf; Art. 38 LSAVerf; Art. 2 Abs. 4 ThürVerf.

17 BGBl. II 2008, S. 1420.

18 Valentin Aichele, Die UN-Behindertenrechtskonvention in der gerichtlichen Praxis, AnwB1 2011, S. 727-730; Eibe Riedel/Michael Arend, Im Zweifel Inklusion. Zuweisung an eine Förderschule 
herangezogen werden, um die entsprechenden Grundrechte des Grundgesetzes auszulegen $^{20}$. Die BRK verdeutlicht, dass der volle und gleichberechtigte Genuss aller Menschenrechte und Grundfreiheiten durch Menschen mit Behinderungen nicht alleine eine Frage des Sozialleistungsrechts ist, sondern als Gestaltungsaufgabe in allen Bereichen von Recht und Politik beachtet werden muss. Die Vertragsstaaten haben sich verpflichtet, alle geeigneten Gesetzgebungs-, Verwaltungs- und sonstigen Maßnahmen der Umsetzung zu treffen ${ }^{21}$ und dabei die Menschen mit Behinderungen durch ihre Verbände aktiv einzubeziehen ${ }^{22}$. Daraus ergibt sich noch keine eindeutige Pflicht zu bestimmten Planungsprozessen auf einer bestimmten Ebene. Die Pflichten, geeignete Informationen zu sammeln (Art. 31 BRK) und eine oder mehrere staatliche Anlaufstellen zu schaffen, die als Koordinierungsmechanismus die Durchführung der entsprechenden Maßnahmen in verschiedenen Bereichen und auf verschiedenen Ebenen erleichtern sollen (Art. 33 BRK), weisen darauf hin, dass der BRK das Konzept eines koordinierten und geplanten Vorgehens zu Grunde liegt. Da Vertragspartner der BRK die nationale Ebene ist - und die Bundesregierung das BMAS als Anlaufstelle benannt hat - kann daraus für eine regionale oder lokale Teilhabeplanung wenig abgeleitet werden. Immerhin wird diese Ebene aber in der BRK mehrfach erwähnt. So wird der Zugang zu gemeindenahen Unterstützungsdiensten als Voraussetzung unabhängiger Lebensführung (Art. 19 lit. b BRK $)^{23}$ und einer an Einbeziehung orientierten Rehabilitation (Art. 26 lit. b BRK) ausdrücklich gefordert. Die Zugänglichkeit von gemeindenahen Dienstleistungen und Einrichtungen für die Allgemeinheit für behinderte Menschen (Art. 19 lit. c BRK) wird ebenfalls ausdrücklich erwähnt. In der Übersetzung von „community support services“24 mit „gemeindenahe Unterstützungsdienste“ ist der Bezug zur örtlichen Gemeinschaft und damit auch zur kommunalen Selbstverwaltung nicht hinreichend wie-

nach Inkrafttreten der BRK, NVwZ 2010, S. 1346; Markus Krajewski, Ein Menschenrecht auf integrativen Schulunterricht, JZ 2010, S. 120.

19 BVerfG, B. v. 14.10.2004, Az. 2 BvR 1481/04, BVerfGE 111, 307.

20 BVerfG, B. v. 23.3.2011, NJW 2011, 2113, Az. 2 BvR 882/09, Rz 52 f.; vgl. BT-Drucks. 16/10808, S. 48.

21 Art. 4 Abs. 1 lit. a BRK; Dazu Theresia Degener, Welche legislativen Herausforderungen bestehen in Bezug auf die nationale Implementierung der UN-Behindertenrechtskonvention in Bund und Ländern?, Behindertenrecht 2009, S. 34-51; Bernd Schulte, Die Behindertenrechtskonvention der Vereinten Nationen - Von der „Integration“ zur „Inklusion“: Änderungsbedarf im deutschen Behindertenrecht, Behindertenrecht 2011, S. 41-48; Peter Masuch, Die UN-Behindertenrechtskonvention anwenden!, in: Christine Hohmann-Dennhardt/ders./Mark Villiger (Hrsg.), Grundrechte und Solidarität, Festschrift für Renate Jaeger, Kehl am Rhein 2011, S. 245-263; Minou Banafsche, Die UNBehindertenrechtskonvention und das deutsche Sozialrecht - eine Vereinbarkeitsanalyse anhand ausgewählter Beispiele (Teil I), SGb 2012, S. 373-379.

22 Art. 4 Abs. 3 BRK.

23 Vgl. Klaus Lachwitz/Peter Trenk-Hinterberger, Zum Einfluss der Behindertenrechtskonvention (BRK) der Vereinten Nationen auf die deutsche Rechtsordnung, Rechtsdienst der Lebenshilfe 2010, S. 45-52.

24 Vgl. ILO, Community Based Rehabilitation - CBR: A Strategy for Rehabilitation, Equalization of Opportunities, Poverty Reduction and Social Inclusion of People with Disabilities, 2005. 
dergegeben. Eine Übersetzung mit „kommunal“ hätte den Bedeutungsgehalt der - nach Art. 50 BRK verbindlichen - englischen Fassung besser wiedergegeben als das gewählte ,gemeindenah“, das in Deutschland zu Unrecht in einem rein geographischen Sinne verstanden werden könnte.

\section{Bundes- und Landesgesetze}

Bundes- und Landesgesetze können eine regionale oder örtliche Teilhabeplanung vorschreiben und regeln, soweit die Bund oder Land zur Gesetzgebung berufen sind. Da der Bund Gemeinden und Gemeindeverbänden keine Aufgaben übertragen darf, kann Bundesgesetzgebung jedoch nur die Länder binden ${ }^{25}$. Diese können möglicherweise verpflichtet sein, die von ihnen landesgesetzlich bestimmten Träger von Aufgaben zur örtlichen oder regionalen Planung zu verpflichten. Diese Träger sind dann häufig Gemeinden oder Gemeindeverbände 26 . So bestehen sozialrechtliche Planungspflichten nach § 19 Abs. 1 SGB IX und § 95 SGB X, die insbesondere Kreise und kreisfreie Städte als Träger der Sozialhilfe betreffen können. Ebenso müssen Teilhabeaspekte vom Aufgabenträger des öffentlichen Nahverkehrs nach § 8 PBefG im Nahverkehrsplan berücksichtigt werden.

Die Länder können für sich selbst, für regionale Landesbehörden wie Regierungsbezirke und für Städte, Kreise und Gemeinden sektorale oder umfassende Pflichten zur Teilhabeplanung regeln. Sie können damit im Bereich ihrer Gesetzgebungskompetenzen umfassend tätig werden und für die Kommunen Pflichtaufgaben - unter Beachtung der finanzverfassungsrechtlichen Vorgaben 27 - statuieren. Angesichts der sich aus Verfassungsrecht und der BRK ergebenden Wertigkeit des Themas und des Erfordernisses, auch behinderten Menschen gleichwertige Lebensverhältnisse und Freizügigkeit im Bundesgebiet zu ermöglichen, wäre eine solche Pflicht kaum als unverhältnismäßiger Eingriff in die kommunale Selbstverwaltung anzusehen 28 .

25 Art. 84 Abs. 1 S. 7 GG; Vgl. Felix Welti/Reza Fakhr Shafaei, Auswirkungen der Reform der bundesstaatlichen Ordnung auf das Recht der Rehabilitation und Teilhabe behinderter Menschen, insbesondere auf die Eingliederungshilfe nach dem SGB XII, in: Bundesverband für evangelische Behindertenhilfe; Bundesverband für Körper- und Mehrfachbehinderte; Bundesvereinigung Lebenshilfe; Caritas Behindertenhilfe und Psychiatrie; Verband für anthroposophische Heilpädagogik; Sozialtherapie und soziale Arbeit (Hrsg.), Föderalismusreform und Behindertenhilfe 2008, S. 79-141.

$26 \S 3$ Abs. 2 SGB XII.

27 Art. 71 Abs. 3 S. 2, 3 BWVerf; Art. 83 Abs. 3 BayVerf; Art. 97 Abs. 3 S. 2 , 3 BbgVerf; Art. 137 Abs. 6 HessVerf; Art. 72 Abs. 3 S. 1, 2 MVVerf; Art. 57 Abs. 4 NdsVerf; Art. 78 Abs. 3 NWVerf; Art. 49 Abs. 5 RhPfVerf; Art. 120 Abs. 1 S. 2 SLVerf; Art. 85 Abs. 2 SächsVerf; Art. 87 Abs. 3 S. 2, 3 LSAVerf; Art. 49 Abs. 2 SHVerf; Art. 93 Abs. 1 S. 2 ThürVerf.

28 Vgl. zur Pflicht, Gleichstellungsbeauftragte für die Gleichstellung der Geschlechter einzuführen: BVerfG v. 26. Oktober 1994, 2 BvR 445/91, BVerfGE 91, 228VerfG LSA v. 12. Dezember 1997, LVG 9/95, LVerfG 7, 284; NdsStGH v. 13. März 1996, StGH 1, 2, 4, 6-20/94, NdsVBl. 1996, 87. 


\section{Teilhabeplanung durch Städte, Gemeinden und Kreise}

\section{Kommunale Selbstverwaltung}

Die Gemeinden, Städte und Kreise sind der rechtlich und politisch vorgegebene Sozialraum oder sie definieren ihn ${ }^{29}$. Eine an der Inklusion in die örtliche Gemeinschaft und in allgemeine Lebenszusammenhänge orientierte Umsetzung des Rechts behinderter Menschen und eine entsprechende Behindertenpolitik müssen daher eine (Re-) Kommunalisierung der Behindertenpolitik diskutieren ${ }^{30}$.

Die Selbstverwaltung der Städte und Gemeinden sowie der Gemeindeverbände, insbesondere der Kreise, ist durch das Verfassungsrecht der Länder ${ }^{31}$ und durch Art. 28 Abs. 2 GG garantiert. Die kommunale Selbstverwaltung umfasst vor allem das Recht, im Rahmen der Gesetze die örtlichen Angelegenheiten selbst zu finden und zu bestimmen, eigenes Satzungsrecht zu geben, eigene Haushaltsmittel auch mit Hilfe eigener Finanzquellen auszugeben und die eigene Verwaltung selbst zu organisieren ${ }^{32}$.

Die kommunalen Hoheiten umfassen auch das Recht, einen umfassenden Planungsprozess zur Teilhabe behinderter Menschen auf dem Gebiet der jeweiligen kommunalen Körperschaft in Gang zu setzen. Dabei sind die Schranken des Landes- und Bundesrechts einzuhalten.

Wenn Art und Umfang der Teilhabeplanung von den Städten, Gemeinden und Kreisen im Rahmen ihrer gewählten Volksvertretungen selbst definiert werden, kann dies vorzugswürdig gegenüber einer nur widerwillig administrierten Pflichtaufgabe sein. Der bis zum Beschluss über Art und Umfang einer Teilhabeplanung notwendige Diskussionsprozess kann bereits Bestandteil notwendiger Prozesse der Bewusstseinsbildung ${ }^{33}$ und Veränderung sein. Zudem kann ein selbst gestalteter Planungsprozess örtliche Besonderheiten und Erfahrungen besser aufgreifen als eine gesetzlich verordnete Planung. Er kann mit der Bau- und Flächenplanung verknüpft werden, die einen gewichtigen Einfluss auf Teilhabechancen hat ${ }^{34}$.

29 Vgl Mario Rund/Ronald Lutz/Tilo Fiegler, Kommunale Teilhabeplanung im Kontext Integrierter Sozialraumplanung Behindertenpolitik, in: Lampke/Rohrmann/Schädler (Fußn. 1), S. 89-104.

30 Albrecht Rohrmann, (Re)kommunalisierung der Behindertenpolitik, in: Lampke/Rohrmann/Schädler (Fußn. 1), S. 25-37.

31 Art. 71 BWVerf; Art. 11 BayVerf; Art. 97 BbgVerf; Art. 144 BremVerf; Art. 137 HessVerf; Art. 72 MVVerf; Art. 57 NdsVerf; Art. 78 NWVerf; Art. 49 RhPfVerf; Art. 117 SLVerf; Art. 84 SächsVerf; Art. 87 LSAVerf; Art. 91 ThürVerf.

32 BVerfG v. 23. November 1988, BVerfGE 79, 127.

33 Art. 8 BRK.

34 Vgl. VGH München v. 4.3.2005, N 04.2100. 
Dagegen ist zu bedenken, dass die Kommunen schon bisher frei waren, eine Teilhabeplanung durchzuführen und dies in ihrer großen Mehrheit nicht getan haben ${ }^{35}$. Da behinderte Menschen eine strukturelle Minderheit sind, bedeutet ein Warten auf den freiwilligen Start, dass die behinderten Menschen auf die Einsicht einer nichtbehinderten Mehrheit angewiesen sind. Unter den Bedingungen einer zum Teil bis zur Notlage angespannten Haushaltslage ist zudem zu befürchten, dass jede freiwillige Aufgabe notleidender Kommunen bis zur Grenze der Vernachlässigung zurückgestellt wird und zurückgestellt werden muss.

Die Länder können die Kommunen verpflichten, Selbstverwaltungsaufgaben zu erfüllen (pflichtige Selbstverwaltungsaufgaben). In diesen Fällen ist nur die Entscheidung über das „Ob“ gesetzlich getroffen, während das „Wie“ den Kommunen überlassen bleibt. Es besteht dann nur eine Rechtsaufsicht des Landes, die den Kommunen erheblichen Spielraum belässt.

\section{Kommunen als Träger staatlicher Aufgaben}

Kommunen können auch von den Ländern als Träger staatlicher Aufgaben bestimmt werden. Je nach Ausgestaltung der Kommunalverfassung in den Ländern können dies Weisungsaufgaben sein, bei denen Landesbehörden die Fachaufsicht übernehmen und so den kommunalen Handlungsspielraum einengen, oder die Kommunen können im Wege der Organleihe vollständig in die Landesverwaltung eingegliedert werden.

Eine Übertragung von Teilhabeplanung als staatliche Weisungsaufgabe kann sinnvoll sein, wenn das Land sich eine fachliche Beteiligung und Standardisierung vorbehalten will, insbesondere weil bei der Planung Bereiche unmittelbarer Landesverwaltung einbezogen werden, wie vor allem der Schulbereich. Auch das für die Barrierefreiheit wichtige Bauordnungsrecht 36 ist kommunale Weisungsaufgabe.

Ebenso wie bei pflichtigen Selbstverwaltungsaufgaben besteht bei der Übertragung von Weisungsaufgaben und bei der Organleihe eine in den Landesverfassungen garantierte und landesrechtlich ausgestaltete Pflicht zum Ausgleich der Kosten, die Konnexität von Aufgabenübertragung und finanziellem Ausgleich ${ }^{37}$. Das gilt allerdings nicht für Aufgaben, die schon bisher bestanden und nur vernachlässigt wurden, wie etwa oft die Überwachung der Barrierefreiheit im Bauordnungsrecht. Auch löst eine Konkretisierung des Stands der Technik in Planungsprozessen noch keine Ausgleichspflicht aus.

35 Vgl. hierzu positiv gewendet: Kerstin Steinfurth, Ein neuer kommunaler Planungsoptimismus und seine Chancen für die Behindertenpolitik, in: Lampke/Rohrmann/Schädler (Fußn. 1), S. 79-88.

36 Joachim Steinbrück, Barrierefreiheit von Wohn- und öffentlich zugänglichen Gebäuden - Regelungsgehalt, Wirkung und Durchsetzung baurechtlicher Bestimmungen, Behindertenrecht 2009, S. 157 ff.; OVG Sachsen-Anhalt v. 16.12.2010, 2 L 246/09 - juris.

37 Vgl. VerfGH Rheinland-Pfalz, Urt. v. 16.3.2001, VGH B 8/00 (Förderung von Betreuungsvereinen). 


\section{Beteiligung behinderter Menschen}

Unabhängig von Art, Ausmaß und Rechtsform der örtlichen Teilhabeplanung ist die Beteiligung behinderter Menschen zu regeln. Sie ist zweckmäßig, um den mehrheitlich nichtbehinderten Entscheidungsträgern in Verwaltung und Selbstverwaltung die Lebenssituation behinderter Menschen zu vermitteln. Sie ist auch durch Art. 4 Abs. 3 BRK geboten, wonach bei Ausarbeitung und Umsetzung von Rechtsvorschriften und politischen Konzepten zur Durchführung der BRK und bei anderen Entscheidungsprozessen, die Menschen mit Behinderungen betreffen, die Vertragsstaaten mit diesen über ihre Organisationen enge Konsultationen führen und sie aktiv einbeziehen müssen. Diese rechtliche Positivierung des Prinzips ,nichts über uns ohne uns“ bindet alle Ebenen der öffentlichen Gewalt. Sie ist eine notwendige partizipative Ergänzung aktivierender Strategien $^{38}$.

Die Form der Beteiligung behinderter Menschen und ihrer Organisationen ist nicht vorgeschrieben. Klar ist jedoch, dass eine rein advokatorische Beteiligung durch einzelne Beauftragte - seien diese behindert oder nicht - nicht ausreicht, da es darauf ankommt, die behinderten Menschen in der Vielfalt ihrer Beeinträchtigungen und Lebenssituationen zu erreichen. Werden Beauftragte eingesetzt, so muss deren Aufgabe sinnvollerweise darin bestehen, die Konsultation mit den behinderten Menschen und ihren Organisationen zu institutionalisieren, also als ständiger Ansprechpartner und Vermittler sowie Interessenvertreter in der Verwaltung zu agieren. Ergänzend oder alternativ zum Konzept des Beauftragten steht die Beteiligung durch Beiräte, in denen typischerweise die Vielfalt behinderter Menschen und ihrer Organisationen abgebildet wird. Sowohl Beauftragte als auch Beiräte sind in den Kommunalverfassungen der Länder als Ausgestaltung der kommunalen Selbstverwaltung zum Teil ausdrücklich zugelassen ${ }^{39}$, anderenfalls können sie mit beratenden Funktionen im Rahmen der Organisationshoheit eingerichtet werden.

Besonders zu beachten ist, dass bei Konsultationen und organisierter Repräsentanz behinderter Menschen bestimmte Gruppen nicht ausgegrenzt werden. Hier sind zunächst die Kinder mit Behinderungen zu nennen, die in Art. 4 Abs. 3 BRK ausdrücklich genannt werden. Sie sind auch, und zwar nicht nur über ihre Eltern und Erziehungsberechtigten, einzubeziehen. Die Beteiligung von Kindern und Jugendlichen ist ihrerseits Gegenstand des Kinder- und Jugendhilferechts ${ }^{40}$ und von Kommunalverfassungen ${ }^{41}$. Sie sollte so ausgestaltet werden, dass behinderte Kinder und Jugendliche an ihr teilhaben. Weiterhin sind zu beachten die jeweils durch eine besondere Situation betroffenen behinderten Frauen, behinderten Migrantinnen und Migranten sowie die seelisch und

38 Michael Spörke, Behindertenpolitik im aktivierenden Staat und ihre Auswirkungen auf die kommunale Ebene, Behindertenpolitik, in: Lampke/Rohrmann/Schädler (Fußn. 1), S. 39-54.

39 Z.B. § 47d GO SH (sonstige Beiräte).

$40 \S 8$ SGB VIII.

41 Z.B. $\S 8 \mathrm{c} \mathrm{HGO}$; $47 \mathrm{f} \mathrm{GO} \mathrm{SH}$. 
geistig behinderten Menschen, die sich zum Teil nicht in traditionellen und bisher üblichen Formen beteiligen können.

\section{Regionale Teilhabeplanung durch Sozialleistungsträger}

\section{Planungsverantwortung nach $S G B$ I und $S G B X$}

Die Sozialleistungsträger sind nach $\S 17$ Abs. 1 SGB I verpflichtet, darauf hinzuwirken, dass jede berechtigte Person die ihr zustehenden Sozialleistungen in zeitgemäßer Weise, umfassend und zügig erhält, die zur Ausführung der Sozialleistungen erforderlichen sozialen Dienste und Einrichtungen rechtzeitig und umfassend zur Verfügung stehen, der Zugang zu den Sozialleistungen möglichst einfach gestaltet wird, ihre Verwaltungs- und Dienstgebäude frei von Zugangs- und Kommunikationsbarrieren sind und Sozialleistungen in barrierefreien Räumen und Anlagen ausgeführt werden. Diese Verantwortung macht deutlich, dass die Sozialleistungsträger keine bloßen „Kostenträger“ sind - das Wort kommt aus gutem Grund im Gesetz nicht vor -, sondern eine umfassende Verantwortung haben, die Zugänglichkeit von Sozialleistungen als Voraussetzung der gesetzlichen Leistungsansprüche zu gewährleisten.

Um dieser Verantwortung gerecht zu werden, haben die Leistungsträger ihre Verwaltung entsprechend zu gestalten, insbesondere im Bereich der Auskunft und Beratung ${ }^{42}$. Je nach spezifischen Rechtsgrundlagen erbringen sie die Sozialleistungen selbst durch eigene Dienste und Einrichtungen oder sie schließen Verträge mit freien und gemeinnützigen oder privaten freiberuflichen oder gewerblichen Leistungserbringern (vgl. § 17 Abs. 1 SGB IX). Diese Verträge sind oft in kollektive Rahmenverträge auf Landesebe$n^{43}$ mit Vergütungs- und Qualitätsregelungen eingebettet, mit denen gleichmäßige, regional und fachlich hinreichende Leistungsangebote gewährleistet werden sollen. Auch die Förderung von Einrichtungen, Diensten und Selbsthilfegruppen kann im Rahmen des Haushaltsrechts ein Instrument der Gewährleistung sein 44 .

Ein weiteres Instrument der Sozialleistungsträger ist die Planung. Nach § 95 SGB X sollen die Sozialleistungsträger Planungen, die auch für die Willensbildung und Durchführung von Aufgaben der anderen Leistungsträger von Bedeutung sind, im Benehmen miteinander abstimmen sowie gemeinsame örtliche und überörtliche Pläne in ihrem Aufgabenbereich über soziale Dienste und Einrichtungen, insbesondere deren Bereit-

42 Vgl. § 22 SGB IX (Gemeinsame Servicestellen); dazu Reza Fakhr Shafaei, Die gemeinsamen Servicestellen für Rehabilitation, 2008; § 92c SGB XI (Pflegestützpunkte); dazu Thomas Klie/Hannes Ziller, Zur Organisationsstruktur von Pflegestützpunkten, NDV 2009, S. 173-178.

$43 \S 21$ Abs. 2 SGB IX; § 78 f SGB VIII; § 75 SGB XI; § 79 SGB XII.

$44 \S 19$ Abs. 5, 29 SGB IX. 
stellung und Inanspruchnahme, anstreben ${ }^{45}$. Die jeweiligen Gebietskörperschaften sowie die gemeinnützigen und freien Einrichtungen und Organisationen sollen hinsichtlich der Bedarfsermittlung beteiligt werden. Auch zu diesem Zweck können nach § 94 SGB X Träger der Sozialversicherung, Verbände von Trägern der Sozialversicherung und die Bundesagentur für Arbeit insbesondere zur gegenseitigen Unterrichtung, Abstimmung, Koordinierung und Förderung der engen Zusammenarbeit im Rahmen der ihnen gesetzlich übertragenen Aufgaben Arbeitsgemeinschaften bilden. Die Träger der unmittelbaren Staatsverwaltung und die Gemeinden, Städte und Kreise als Sozialleistungsträger sind hier deshalb nicht genannt, weil ihre Verwaltungsorganisation alleine in die Gesetzgebungskompetenz der Länder fällt. Auch insoweit wäre es zweckmäßig, die Zusammenarbeit von Sozialleistungsträgern zum Zwecke der Planung - auch Teilhabeplanung - landesgesetzlich auszugestalten.

\section{Planungsverantwortung für Leistungen zur Teilhabe nach SGB IX}

Verdeutlicht wird die Planungsverantwortung der Sozialleistungsträger für die Rehabilitationsträger als Träger der Leistungen zur Teilhabe in $\S 19$ Abs. 1 SGB IX. Danach sind diese verpflichtet, gemeinsam unter Beteiligung der Bundesregierung und der Landesregierung darauf hinzuwirken, dass die fachlich und regional erforderlichen Rehabilitationsdienste und -einrichtungen in ausreichender Zahl und Qualität zur Verfügung stehen.

Problematisch für die Praxis ist, dass die Rehabilitationsträger - die gesetzlichen Krankenkassen, die Träger der gesetzlichen Rentenversicherung, die Träger der gesetzlichen Unfallversicherung, die Bundesagentur für Arbeit sowie die nach Landesrecht bestimmten Träger der sozialen Entschädigung, der Kinder- und Jugendhilfe und der Sozialhilfe - bislang über keine gemeinsamen Strukturen verfügen, in denen sie der Planungsverantwortung nachkommen können. Das gegliederte System der Leistungen zur Teilhabe ist nicht nur vertikal auf sieben Gruppen von Rehabilitationsträgern verteilt, die jeweils nach eigenen Grundsätzen die Infrastruktur ihrer Leistungen planen oder auch nicht -, es ist auch horizontal so gegliedert, dass die einen Träger vorrangig auf Bundesebene agieren - wie die Bundesagentur -, andere auf Bundes- und Landesebene in einer zum Teil konkurrierenden Binnengliederung - wie Rentenversicherung und Krankenkassen - und wieder andere verteilt auf die Landes- und Kommunalebene wie Sozialhilfe und Jugendhilfe. Einen in den Gemeinden präsenten Verwaltungsunterbau haben längst nicht alle Träger. Vielmehr haben Fusionen und Zentralisierung dazu geführt, dass sich viele von ihnen aus der örtlichen Ebene zurückgezogen haben und mit Bürgern und Leistungserbringern nicht mehr unmittelbar kommunizieren.

45 Vgl. Ernst-Wilhelm Luthe, Sozialplanungsrecht, ZSR 1994, S. 762-780, 838-855. 
Die Bundesarbeitsgemeinschaft für Rehabilitation ist als privatrechtlicher Verein öffentlich-rechtlicher und privatrechtlicher Akteure ausgestaltet, der als Rahmen für Einigungsprozesse der Rehabilitationsträger im Rechtsraum des Bundes dient. Dagegen können die Länder und die kommunalen Sozialleistungsträger sich zwar aus eigener Einsicht an diesen Prozessen beteiligen, können aber bundesrechtlich nur gebunden werden, sich an ihnen zu orientieren (§ 13 Abs. 6 SGB IX).

In $\S 12$ Abs. 2 SGB IX ist geregelt, dass die Rehabilitationsträger regionale Arbeitsgemeinschaften bilden sollen. Diese Arbeitsgemeinschaften wären geeignet, die Planung der Dienste und Einrichtungen für Leistungen zur Teilhabe auf regionaler und örtlicher Ebene $\mathrm{zu}$ operationalisieren. Bislang sind regionale Arbeitsgemeinschaften nicht gebildet worden. Nötig wären entweder freiwillige Entscheidungen der einzelnen Rehabilitationsträger oder landesrechtliche Regelungen, die zwar möglich ${ }^{46}$, bislang aber unterblieben sind. Spätestens seit der Föderalismusreform kann das SGB IX nicht ohne aktive Beteiligung der Länder umgesetzt werden.

\section{Planungsverantwortung für Pflegeleistungen nach SGB XI}

Die Sicherstellungsverantwortung für die Leistungen der Pflegekassen liegt nach $\S 69$ SGB XI bei den Pflegekassen, die verpflichtet sind, eine bedarfsgerechte und gleichmäßige, dem allgemein anerkannten Stand medizinisch-pflegerischer Erkenntnisse entsprechende pflegerische Versorgung der Versicherten zu gewährleisten ${ }^{47}$. Als Instrumente hierzu sind benannt Versorgungsverträge, Leistungs- und Qualitätsvereinbarungen sowie Vergütungsvereinbarungen mit den Trägern von Pflegeeinrichtungen und sonstigen Leistungserbringern. Die Pflegekassen sind aber nicht die einzigen Träger von Pflegeleistungen, sondern werden hierbei von den Trägern der Sozialhilfe ergänzt (§ 61 SGB XII).

Nach $\S 8$ Abs. 2 SGB XI wirken die Länder, die Kommunen, die Pflegeeinrichtungen und die Pflegekassen unter Beteiligung des Medizinischen Dienstes der Krankenversicherung eng zusammen, um eine leistungsfähige, regional gegliederte, ortsnahe und aufeinander abgestimmte ambulante und stationäre pflegerische Versorgung der Bevölkerung zu gewährleisten. Sie sollen danach zum Ausbau und zur Weiterentwicklung der notwendigen pflegerischen Versorgungsstrukturen beitragen; das soll insbesondere für die Ergänzung des Angebots an häuslicher und stationärer Pflege durch neue Formen der teilstationären Pflege und Kurzzeitpflege sowie für die Vorhaltung eines Angebots von die Pflege ergänzenden Leistungen zur medizinischen Rehabilitation gelten. Sie sollen darüber hinaus die Bereitschaft zu einer humanen Pflege und Be-

46 Vgl. Welti/Shafaei (Fußn. 25), S. 125.

47 Vgl. Felix Welti, Qualitätssicherung, Fallmanagement und räumliche Versorgungsstrukturen, in: Karl-Jürgen Bieback (Hrsg.), Die Reform der Pflegeversicherung 2008, Berlin 2009; Silke Hamdorf, Öffentliche und private Verantwortung für Qualität in der Pflege, Berlin 2009. 
treuung durch hauptberufliche und ehrenamtliche Pflegekräfte sowie durch Angehörige, Nachbarn und Selbsthilfegruppen unterstützen und fördern und so auf eine neue Kultur des Helfens und der mitmenschlichen Zuwendung hinwirken. Diese allgemeine bundesrechtliche Zielvorgabe ist in den Pflegegesetzen der Länder in unterschiedlicher Weise operationalisiert worden. Überwiegend ist eine kommunale Pflegeplanung ausdrücklich vorgesehen ${ }^{48}$, die in eine umfassendere Teilhabeplanung einbezogen werden müsste.

Da Menschen mit Pflegebedarf eine Teilgruppe behinderter Menschen sind, ist die örtliche Pflegeplanung ein wichtiger Teilbereich von Teilhabeplanung. Die bisherige Trennung der Regelungsbereiche von Teilhabe und Pflege im Sozialrecht sollte nicht dazu führen, die notwendigen Überschneidungen und Wechselwirkungen zwischen der notwendigen Infrastruktur von Diensten, Einrichtungen und Selbsthilfe aus dem Blick zu nehmen 49 . Sie kann auch die Verbindung zu Planungsprozessen im Sinne älterer Menschen ${ }^{50}$ herstellen.

\section{Gesundheitsplanung}

Die Sicherstellungsverantwortung für die allgemeine gesundheitliche Versorgung liegt in Bezug auf Krankenhäuser bei den Ländern, die nach ihren Krankenhausgesetzen Krankenhauspläne erstellen, bei denen die Gemeinden und Kreise zumeist beteiligt sind. Für die vertragsärztliche Versorgung liegt der Auftrag zur Sicherstellung bei den Kassenärztlichen Vereinigungen ( 72 SGB V), die darüber Verträge mit den Krankenkassen auf Landesebene schließen. Für nichtärztliche Teile der Krankenbehandlung wie etwa die Versorgung mit Hilfsmitteln und Heilmitteln liegt die Verantwortlichkeit bei den Krankenkassen. Diese uneinheitliche Verantwortung im Bereich der Krankenbehandlung führt zu Abstimmungsproblemen. Sie ist nicht günstig für eine umfassend an den besonderen Bedürfnissen chronisch kranker und behinderter Versicherter orientierte Infrastruktur (vgl. § 2a SGB V). Eine ergänzende Funktion in der Gesundheitsversorgung hat der landesrechtlich geregelte öffentliche Gesundheitsdienst, der meist auf örtlicher Ebene von den Kreisen und kreisfreien Städten getragen wird. Ihm wird in einigen Landesgesetzen eine Planungsfunktion zugeschrieben ${ }^{51}$.

48 § 6 PfG NW; § 4 Pflege-V-AG SN; § 2 ThürAG PflegeVG; § 5 Abs. 2 LPflegeG M-V; §§ 4, 5 NPflegeG; § 2 LPflegeASG Rheinland-Pfalz; § 4 Abs. 2 HessAG PflegeVG; § 2 HmbLPG; § 4 BremAGPflegeVG; § 4 LPflgG BW; § 2 LPflegEG Berlin; § 3 LPflegeG SH; Art. 69 AGSG Bayern.

49 Vgl. Felix Welti, Bietet das SGB IX einen geeigneten Rahmen für Teilhabe und Pflege?, Archiv für Wissenschaft und Praxis der sozialen Arbeit 2010, H. 3, S. 46-62.

50 Vgl. Cordula Barth, Örtliche Teilhabeplanung für ältere Menschen mit und ohne Behinderung Behindertenpolitik, in: Lampke/Rohrmann/Schädler (Fußn. 1), S. 257-268.

$51 \S 6$ GDG Berlin; § 24 ÖGDG NRW; § 9 Abs. 3 BbgGDG; § 12 ÖGDG Bremen; § 5 HmbGDG; $\S 12$ GDG LSA. 


\section{Schluss}

Insgesamt ist eine regionale und örtliche Teilhabeplanung ein zweckmäßiges, jedenfalls im Bereich der Sozialleistungen auch notwendiges und im Gesetz angelegtes, wenn auch nicht ausgeformtes Instrument, um die staatliche und öffentliche Verantwortung für behinderte Menschen wahrzunehmen. Teilhabeplanung kann aus der kommunalen Selbstverwaltung heraus angeregt und entwickelt werden. Dabei sollte an die bestehenden kommunalen Kompetenzen in der Pflege- und Gesundheitsplanung angeknüpft werden. Um umfassend wirksam zu werden, sind aber auch die nicht-kommunalen Rehabilitationsträger und die Pflegekassen sowie Landes- und Bundesbehörden einzubeziehen.

Politisch sinnvoll wäre es daher, die örtliche und regionale Teilhabeplanung im Bundesrecht - am Besten im SGB IX - explizit zu verankern. Dabei müsste zugleich verdeutlicht werden, dass die Länder die nähere Ausgestaltung regeln können und müssen. Auf eine solche Regelung sollte aber nicht gewartet werden, denn Handlungsbedarf und Handlungsmöglichkeiten bestehen schon heute. 


\title{
Barrieren im Sozialraum - ein Kommentar
}

\author{
Markus Schäfers
}

$\begin{array}{ll}\text { I. Einführung } & 101\end{array}$

II. Teilhabeförderliche Umwelt schaffen 102

III. Unterstützungssysteme reformieren 103

IV. Positives Bild von Behinderung in der Öffentlichkeit vermitteln 104

$\begin{array}{ll}\text { V. Literatur } & 105\end{array}$

\section{Einführung}

Wie lassen sich teilhabeförderliche Umwelten schaffen? Moderne fachliche und sozialpolitische Konzepte richten ihren Blick - stärker als in der Vergangenheit - auf die Umwelten, in denen Menschen mit Behinderung leben und ihren Alltag zu bewältigen versuchen. Damit wird den sozialen Einflussgrößen bei der Entstehung und Ausprägung von Behinderung größerer Stellenwert eingeräumt. Konkrete Umwelten umfassen nicht nur die baulich-materielle Umgebung, sondern auch Aspekte wie die Zugänglichkeit von Informationen, die Einstellung und das Verhalten von Mitbürgerinnen und Mitbürgern gegenüber Menschen mit Behinderung oder die Beschäftigungspolitik von Arbeitgebern.

Insofern erscheint es vielversprechend, bei der Frage danach, wie sich die Möglichkeitsräume von Menschen mit Behinderung zur gesellschaftlichen Teilhabe erweitern lassen, bei den vielfältigen gesellschaftlich geprägten Umweltbarrieren anzusetzen. Welche Zielrichtungen, Strategien und Konzepte erscheinen dabei zukunftsweisend?

Ich möchte von drei Thesen ausgehen, um zentrale Herausforderungen, die mit dem Thema „Inklusionsbarrieren“ zusammenhängen, zu identifizieren und da, wo es möglich erscheint, Ansätze zur Lösung und Überwindung folgender Herausforderungen abzuleiten:

1. Die „Inklusionsfähigkeit“ der Gesellschaft ist begrenzt

2. Traditionelle Hilfesysteme werden als ineffektiv beurteilt und als Instanzen, die selbst exkludierend wirken 
3. Das Bild von Behinderung in der Öffentlichkeit festigt einstellungsbedingte Barrieren

\section{Teilhabeförderliche Umwelt schaffen}

Sozialraumorientierung, Regionalplanung, örtliche Teilhabeplanung - all diese Konzepte und Instrumente laufen darauf hinaus, eine inklusive bzw. teilhabeförderliche Umwelt zu schaffen, in denen Menschen mit unterschiedlichen Voraussetzungen und Bedürfnissen ein Leben nach ihren Vorstellungen führen können ${ }^{1}$.

Inwiefern sozialräumlich angelegte Konzepte dabei der Schlüssel zum Erfolg sein können, wird insbesondere in der Behindertenhilfe kontrovers diskutiert: Von manchen wird Sozialraumorientierung als inklusionsförderliche Innovation betrachtet, das die Verbesonderung von Hilfen zu überwinden verspricht ${ }^{2}$, von anderen als Sparmodell disqualifiziert, das ein Recht des Einzelnen auf bedarfsgerechte Unterstützung zu unterlaufen versucht ${ }^{3}$.

In der Vergangenheit hat sich die traditionelle Behindertenhilfe ambivalent verhalten: Einerseits hat sie die begrenzte „Inklusionsfähigkeit“ der gesellschaftlichen Regelsysteme kritisiert und die damit einhergehenden Exklusionsmechanismen im Bildungsbereich, auf dem Arbeitsmarkt, im kulturellen Leben usw. Andererseits hat sie sich selbst als Sondersystem entwickelt, die Regelsysteme von ihren Aufgaben entlastet und so paradoxerweise dazu beigetragen, dass Menschen mit Behinderung aus gesellschaftlich relevanten Zusammenhängen der Lebensführung weitgehend ausgeschlossen und ,unsichtbar" wurden 4 .

Mit der Zielbestimmung, gesellschaftliche Teilhabe zu fördern, gilt es nun für die Behindertenhilfe, sich stärker in der Gesellschaft zu verorten, mit ihren Aktivitäten und Kompetenzen das Gemeinwesen mitzugestalten und vor Ort präventiv und unterstützend zu wirken.

Sozialraumorientiert zu handeln, fallübergreifend zu denken und die Sozialräume, in denen sich Menschen bewegen, als vornehmliches Handlungsfeld zu begreifen, entspricht nicht der Tradition und Kultur der Behindertenhilfe und ist nicht durch rechtliche und finanzielle Bedingungen unterlegt. Das Leistungsrecht ist durch das Bedarfsdeckungsprinzip des Einzelnen geprägt und durch einen individuellen Rechtsanspruch auf Hilfe. Wie lässt sich so sozialräumliche Arbeit organisieren und nachhaltig finanzieren?

1 Ausführlich zu einer örtlichen Teilhabeplanung Welti, S. 87 in diesem Band.

2 Hinte, Innovation oder Depression - Zum Dilemma der Diskussion um Sozialraumorientierung, Teilhabe 2/2012, S. 66.

3 Dahme/Wohlfahrt, Der Sozialraum als Rettungsanker des Sozialstaats mit antikapitalistischer Durchschlagskraft?, Teilhabe 2/2012, S. 69.

4 Wacker/Wansing/Schäfers, Personenbezogene Unterstützung und Lebensqualität, Teilhabe mit einem Persönlichen Budget, Wiesbaden 2005, S. 17. 
Leistungsanbieter haben nur dann einen (wirtschaftlichen) Vorteil, wenn möglichst viele Hilfefälle produziert und bearbeitet werden. Sozialraumorientiertes Handeln hingegen versucht, eine Typisierung und Unterscheidung zwischen hilfebedürftig/nicht hilfebedürftig möglichst zu vermeiden.

Zwar gibt es auch in der Behindertenhilfe bereits jetzt schon wegweisende und erfolgreiche Konzepte sozialräumlichen Handelns, indem beispielsweise in einer Region Arbeitsplätze für Menschen mit Behinderung geschaffen werden durch Netzwerkarbeit, Patenmodelle und individuelles Job-Coaching ${ }^{5}$. Diese Ansätze arbeiten im Moment gegen die bestehenden rechtlichen und finanziellen Strukturen und werden nicht von ihnen gestützt. So wird es schwer sein, sie voll zum Tragen kommen zu lassen und weiter zu verbreiten.

\section{Unterstützungssysteme reformieren}

In der sozialpolitischen Diskussion um die Weiterentwicklung der Unterstützungssysteme, insbesondere im Kontext der Reform der Eingliederungshilfe, steht aber nicht der Sozialraum im Zentrum der Überlegungen, sondern der Begriff der „Personzentrierung". Personzentrierte Hilfe wird verstanden und (verkürzt) dekliniert als Fokussierung auf den Bedarf des Einzelnen, Bewilligung der Leistungen unabhängig vom Ort und der Form der Leistungserbringung (Aufhebung der Trennung zwischen ambulant und stationär), Unterscheidung der Fachleistung von existenzsichernden Leistungen und ein personenbezogenes Fallmanagement des Sozialhilfeträgers ${ }^{6}$.

Die Umstellung von der Angebots- zur Personzentrierung ist programmatisch ein Schritt in die richtige Richtung, aber nicht ausreichend. Der Wirkkraft einer ausschließlichen Beschäftigung mit dem Einzelnen, seinen Kompetenzen und Defiziten sind Grenzen gesetzt; Probleme werden weiterhin vorwiegend im Individuum verortet, die Umweltbedingungen werden so nicht hinreichend in den Blick genommen. Dazu braucht es einen ergänzenden Blick auf die Infrastruktur und die Gegebenheiten des Sozialraums.

Wenn es um die Frage geht, in welche Richtung das Wohlfahrtssystem gelenkt werden soll, wird unter dem Stichwort „Personzentrierung“ als wesentliche Stellschraube immer wieder das Wunsch- und Wahlrecht des Leistungsberechtigten hervorgehoben. Hier herrscht erstaunliche Einigkeit bei allen Akteuren, von der Selbsthilfe, den Leistungsanbietern bis zu den Leistungsträgern.

5 Basener/Häußler, Bamberg bewegt, Integration in den Arbeitsmarkt: Eine Region wird aktiv, Hamburg 2008.

6 ASMK - Bund-Länder-Arbeitsgruppe „Weiterentwicklung der Eingliederungshilfe für Menschen mit Behinderungen" der ASMK, Eckpunkte für die Reformgesetzgebung Eingliederungshilfe im SGB XII, 2009, abrufbar unter http://www.alle-inklusive.de/wp-content/uploads/2009/12/ASMK2009-Anlage-1-Eckpunkte-Eingliederungshilfe.pdf. 
Das Wunsch- und Wahlrecht zu stärken und Auswahlmöglichkeiten zu schaffen, ist sicherlich richtig und wichtig, dagegen lässt sich kaum etwas glaubhaft einwenden. Allerdings besteht die Gefahr, dass die Bedeutung des Wunsch- und Wahlrechts im Kontext dieser Diskussion überformt wird. Sie darf nicht hochstilisiert werden zum einzigen Regulativ für die Weiterentwicklung der Unterstützungssysteme insgesamt.

Kritisch lässt sich einwenden, dass sich das Wunsch- und Wahlrecht zur Legitimation jedweder Angebotsentwicklung missbrauchen lässt - salopp gesagt nach dem Motto: „Hauptsache, es bestehen Alternativen“. Gerade das, was als Alternativen zur Verfügung steht, präsentiert sich aber immer schon so, wie es gesellschaftlich-historisch entstanden ist (z. B. in Bezug auf die stationäre und ambulante Angebotsstruktur in einer Region). Zudem ist das Wunsch- und Wahlrecht selbst zeitlich und kulturell eingebunden 7; was Menschen mit Behinderung und ihre Familien heute wollen, ist Ausdruck dessen, was sie in der Vergangenheit erfahren haben und in der Gegenwart bilanzieren. Zugleich stellt sich die Frage, ob das Wunsch- und Wahlrecht repräsentativ und damit geeignet ist, zu einem Ausgleich unterschiedlicher Interessen beizutragen: Schließlich ist davon auszugehen, dass nicht alle Menschen mit Behinderung unabhängig von der Schwere ihrer Beeinträchtigung in gleichem Maße die Chance haben, bei Entscheidungen, die sie betreffen, gehört zu werden und ihr Recht durchzusetzen, z. B. wenn es um die Auswahl und Gestaltung von Wohn- und Betreuungsformen geht ${ }^{8}$.

Die Gesamtverantwortung, teilhabeförderliche Bedingungen zu schaffen und geeignete Unterstützungssysteme bereitzustellen, ist eine staatliche. Um diese Entwicklungsaufgabe meistern zu können, ist eine Vorstellung davon erforderlich, wie das Wohlfahrtssystem in den nächsten Jahren und Jahrzehnten gestaltet sein muss, um die Bedarfe zukünftiger Nutzerinnen und Nutzer der Unterstützungsleistungen zu decken und gesellschaftliche Herausforderungen bewältigen zu können. In diesem Zusammenhang kommt dem Wunsch- und Wahlrecht sicherlich eine gewisse Bedeutung zu, aber diese Verantwortung lässt sich nicht an den Einzelnen und sein Wunsch- und Wahlrecht delegieren.

\section{Positives Bild von Behinderung in der Öffentlichkeit vermitteln}

Eine Neuorientierung des Hilfesystems allein wird nicht ausreichend sein, um teilhabeförderliche Umwelten zu schaffen. Teilhabe beruht ganz wesentlich auf sozialen Austauschprozessen, dazu sind gelingende Anerkennungsprozesse erforderlich ${ }^{9}$. Hier wird die Begrenztheit professionellen Handelns deutlich. Allerdings kann es durchaus fachli-

7 Wocken, Elternwahlrecht!? Über Dienstbarkeit, Endlichkeit und Widersinn des Elternwillens, Vierteljahresschrift für Heilpädagogik und ihre Nachbargebiete 3/2010, S. 186.

8 Dazu Wansing, S. 69 in diesem Band.

9 Dazu Dederich, S. 61 in diesem Band. 
che Aufgabe sein, günstige Voraussetzungen für gelingende Anerkennungsprozesse zu schaffen. Dies ist jedoch insofern widersprüchlich, als Unterstützungsleistungen für bestimmte Personengruppen die Andersartigkeit der Adressaten markieren können und so zur Verstärkung des Problems beitragen, dessen Lösung sie eigentlich intendieren.

Ein Ansatzpunkt kann darin liegen, das öffentliche Bild von Behinderung, das im Allgemeinen auf festgefahrenen Stereotypen basiert, in positiver Weise zu verstören. Dadurch lässt sich $u$. U. die feste Grenzziehung zwischen behindert/nicht behindert aufbrechen. Ein Beispiel hierfür ist das bürgerschaftliche Engagement von Menschen mit Behinderung, die sich selbst im Gemeinwesen ehrenamtlich für andere Menschen oder für die Ziele einer Organisation einsetzen, z. B. in Tafel-Projekten, im Naturschutz oder in der Freiwilligen Feuerwehr ${ }^{10}$. Dadurch werden sie nicht mehr ausschließlich in der Rolle des Hilfeempfängers gesehen, sondern als wertvolle Mitbürger, die auch etwas für die Gesellschaft leisten können.

\section{Literatur}

ASMK - Bund-Länder-Arbeitsgruppe „Weiterentwicklung der Eingliederungshilfe für Menschen mit Behinderungen“ der ASMK, Eckpunkte für die Reformgesetzgebung Eingliederungshilfe im SGB XII, 2009, http://www.alle-inklusive.de/wp-content/uploads/2009/12/ ASMK-2009-Anlage-1-Eckpunkte-Eingliederungshilfe.pdf (abgerufen am 07.10.2012).

Basener, Dieter/Häußler, Silke, Bamberg bewegt, Integration in den Arbeitsmarkt: Eine Region wird aktiv, Hamburg 2008.

Dahme, Heinz-Jürgen/Wohlfahrt, Norbert, Der Sozialraum als Rettungsanker des Sozialstaats mit antikapitalistischer Durchschlagskraft?, Teilhabe 51 (2) 2012, S. 69.

Hinte, Wolfgang, Innovation oder Depression - Zum Dilemma der Diskussion um Sozialraumorientierung, Teilhabe 51 (2) 2012, S. 66.

Wacker, Elisabeth/Wansing, Gudrun/Schäfers, Markus, Personenbezogene Unterstützung und Lebensqualität, Teilhabe mit einem Persönlichen Budget, Wiesbaden 2005.

Wocken, Hans, Elternwahlrecht!? Über Dienstbarkeit, Endlichkeit und Widersinn des Elternwillens, Vierteljahresschrift für Heilpädagogik und ihre Nachbargebiete 79 (3) 2010, S. 186.

10 Vgl. www.lebenshilfe-aktiv.de. 


\title{
Inklusion im Sozialraum aus Sicht des Deutschen Vereins
}

\author{
Andreas Kuhn
}

I. Inklusion im Sozialraum aus Sicht des Deutschen Vereins 107

$\begin{array}{ll}\text { II. Soziale Inklusion } & 108\end{array}$

III. Barriere Wertschätzung? $\quad 111$

$\begin{array}{ll}\text { IV. Literatur } & 114\end{array}$

\section{Inklusion im Sozialraum aus Sicht des Deutschen Vereins}

Die UN-Behindertenrechtskonvention hat dazu beigetragen, dass auch im Deutschen Verein das Thema Inklusion intensiv diskutiert wird und Papiere beschlossen wurden, die beschreiben, wie Inklusion zu verwirklichen ist: ein Diskussionspapier zu inklusiver Bildung, Eckpunkte für einen inklusiven Sozialraum und Empfehlungen zu einer örtlichen Teilhabeplanung für ein inklusives Gemeinwesen.

Auch zum Sozialraum hat sich der Deutsche Verein in praktischer Absicht geäußert 1 . Soziale Räume sind als - politisch oder statistisch definierte - Gebiete im Gemeinwesen zu verstehen, als Wohnquartiere und Stadtteile, in denen die soziale Integration der darin lebenden Bewohner und Bewohnerinnen stattfindet. Soziale Räume sind Gebiete im Gemeinwesen, die auch heute die Sozialisation, die persönliche Entwicklung, die Lebensbedingungen und die Lebensweise der darin aufwachsenden und lebenden Individuen beeinflussen ${ }^{2}$. In welchem Ausmaß, ist natürlich individuell unterschiedlich. Generell gilt: Je geringer die Mobilität, desto wichtiger ist der so definierte Sozialraum. Ein zweiter Einflussfaktor ist die residentielle Segregation insbesondere von ethnischreligiösen Gruppen.

Inklusion und Sozialraum in Zusammenhang gebracht haben die Arbeits- und Sozialminister auf ihrer Konferenz (ASMK) im November 2009. Die Konferenz stellt fest:

1 Und unterscheidet sich damit deutlich von den Debatten, die in Deutschland über den Begriff des Sozialen Raums geführt wurden; vgl. z.B. Kessel u.a. (Hrsg.), Handbuch Sozialraum, Wiesbaden 2005.

2 Eckpunkte des Deutschen Vereins zur sozialräumlichen Ausgestaltung kommunalen Handelns, NDV 9/2008, S. $377 \mathrm{ff}$. 
Die Weiterentwicklung der Eingliederungshilfe kann ihre volle Wirkung nur dann entfalten, wenn sie sozialräumlich unterstützt wird (Beschluss der Konferenz vom 25./26.11.2009), und belässt es bei dieser - plausiblen - Annahme. Bei der Weiterentwicklung der Eingliederungshilfe soll Inklusion als Leitidee umgesetzt werden, Hilfe soll personenzentriert sein (nicht einrichtungszentriert). Zum Auf- und Ausbau inklusiver Sozialräume sollen Handlungsstrategien erarbeitet werden, und die Weiterentwicklung der Eingliederungshilfe soll insgesamt kostenneutral sein.

In diesem Beitrag will ich aus der pragmatischen Sicht des Deutschen Vereins und aus Sicht der Stadtsoziologie sondieren, welche Bedeutung der Sozialraum für Inklusion hat und auf die These eingehen, dass inklusive Sozialräume nur in dem Maße verwirklicht werden können, wie den Menschen mit Behinderung ein „positiver Wert“ zuerkannt wird.

\section{Soziale Inklusion}

Der Deutsche Verein definiert inklusiven Sozialraum als ein barrierefreies Lebensumfeld, das alle Menschen mit und ohne Behinderungen, alte und junge Menschen, Menschen mit oder ohne Migrationshintergrund selbstbestimmt gemeinsam nutzen und mitgestalten können. Nach dieser Definition sind die sachlichen und materiellen Bedingungen der entscheidende Faktor dafür, in welchem Maße ein Sozialraum Möglichkeiten für ein inklusives Leben bietet. Und Inklusion ist ein Thema für alle Menschen in einem Sozialraum, nicht nur für Menschen mit Behinderung. $\mathrm{Zu}$ einem inklusiven Sozialraum gehört auch, dass es dort Beratungs- und Unterstützungsstrukturen gibt und Netzwerke, die für alle Interessierten offen sind und zur Beteiligung einladen. Das ist nur möglich, wenn sich die Bewohner und Bewohnerinnen gegenseitig akzeptieren und Vielfalt in ihrem Lebensumfeld wertschätzen. Beteiligung an Planungs-, Gestaltungsund Entscheidungsprozessen ist ein weiteres wesentliches Merkmal inklusiver Sozialräume. In einem inklusiven Sozialraum werden Kinder- und Jugendliche mit und ohne Handicap von Anfang an gemeinsam von den zuständigen Institutionen gefördert und unterstützt.

Die ASMK hat in ihren Eckpunkten zur Weiterentwicklung der Eingliederungshilfe (Oktober 2009) ähnliche Elemente für einen inklusiven Sozialraum benannt. Die ASMK betont, dass es um wohnortnahe Strukturen und eine Vielfalt an Wohnformen geht und Unterstützung auch von ehrenamtlichen Strukturen und einem Hilfe-Mix getragen wird.

Die Empfehlungen für eine örtliche Teilhabeplanung stimmen mit diesen Elementen überein. Sie werden in drei Zielen für die Planung zusammengefasst: die Herstellung einer barrierefreien öffentlichen Infrastruktur, die Entwicklung eines an Inklusion ausgerichteten Hilfesystems und die Sensibilisierung der Öffentlichkeit für Diskriminierungsrisiken und damit verbunden die Schaffung eines Solidaritätsbewusstseins. 
Die ASMK und auch der Deutsche Verein benennen die wichtigen Elemente eines inklusiven Sozialraums. Die Papiere sind in dieser Hinsicht wahrscheinlich sehr instruktiv. Die Elemente werden jedoch nicht in eine Rangfolge gebracht. Natürlich könnten alle Elemente auch als „Barrieren“ für Inklusion interpretiert, sozusagen mit negativen Vorzeichen versehen werden. Aber die Papiere verzichten darauf, Barrieren für einen inklusiven Sozialraum so anzusprechen, wie das die Rehabilitationswissenschaft und Behindertenpädagogik doch sehr dezidiert tut, als Defizite eines Gemeinwesens oder einer Kommune, unter denen besonders Menschen mit Behinderung zu leiden haben. Obwohl Barrieren eindeutig ein wesentlicher Aspekt für Inklusion im Sozialraum sind, werden diese von der ASMK und auch vom Deutschen Verein nicht definiert, differenziert und erörtert. Das ist vielleicht ein deutliches Manko und müsste bei weiteren Empfehlungen aufgearbeitet werden. ${ }^{3}$ Auch dann sind „Barrieren“ jedoch ein analytisches Konzept und eine orientierende Kategorie, kein normatives Verdikt.

Sowohl die sozialpolitischen Akteure wie die Experten für Menschen mit Behinderung unterstellen, dass Sozialräume relevant sind für Inklusion ${ }^{4}$. Das ist - wie gesagt plausibel, aber es wäre natürlich gut zu wissen, welche Strukturen eines Sozialraums und welche Mechanismen in Sozialräumen es sind, die Inklusion befördern, erschweren oder verhindern. Und es könnte angezeigt sein, den Begriff „Inklusion“ zu differenzieren, um Zusammenhänge mit dem Sozialraum zu beschreiben und zu analysieren. Genau dieser Aufgabe hat sich ein Forschungsprojekt im Auftrag des Bundesarbeitsministeriums gewidmet, das Möglichkeiten der verbesserten sozialen Inklusion in der Wohnumgebung untersucht hat. 5

Die Bezeichnung „Sozialraum“ wird oft synonym mit „Stadtteil“, „Ortsteil“, „Quartier“ oder „Wohnumgebung“ verwendet. Damit gemeint sind „kleinräumige Einheiten“, die durch den geografischen Raum wie durch die soziale Zusammensetzung der Bewohnerschaft geprägt werden. So verstanden, lassen sich drei Eigenschaften bzw. Mechanismen in Sozialräumen feststellen, die Inklusion maßgeblich beeinflussen:

- die Infrastrukturausstattung und -qualität (insbesondere Bildungseinrichtungen und das Gesundheitssystem, auch Sport- und Freizeiteinrichtungen sowie soziale Dienste), die Verkehrssituation und bauliche Struktur. Vor allem die Schulen

3 Eine sehr gute fachliche Grundlage dafür bieten die Ergebnisse des Sondergutachtens „Barrierefreie Stadtquartiere“, das im ExWoSt-Forschungsfeld "Innovationen für familien- und altengerechte Stadtquartiere" des BMVBS entstanden ist und im August 2011 veröffentlicht wurde; vgl. Bauer/Böcker/Rohlwing/Dähner, Barrierefreie Stadtquartiere, ExWoSt-Sondergutachten im Auftrag des Bundesministeriums für Verkehr, Bau und Stadtentwicklung, Berlin 2011.

4 Und den politisch-pragmatischen Papieren ist nachzusehen, dass Inklusion stark tautologisch positioniert wird, wenig systematisch; dass z.B. ein an Inklusion ausgerichtetes Hilfesystem ein inklusives Gemeinwesen auszeichnet.

5 Häussermann/Schwarze/Jaedicke/Bär/Bugenhagen, Möglichkeiten der verbesserten sozialen Inklusion in der Wohnumgebung, Forschungsprojekt im Auftrag des Bundesministeriums für Arbeit und Sozialordnung, Schlussbericht, Berlin 2010. 
verstärken oder kompensieren Benachteiligungen, die sich aus den sozialen Beziehungen (s.u.) ergeben;

- soziale Beziehungen, die das informelle Lernen (insbesondere über Peers) und die sozialen Netzwerke (oft über die Verwandtschaft) bestimmen. Soziale Beziehungen vermitteln Normen und Vorbilder und beeinflussen darüber das Denken und Verhalten in einem Sozialraum (,kollektive Sozialisation“)6. Je nachdem wie homogen oder heterogen ein soziales Netz ist, schränkt dieses Erfahrungs- und Kooperationsmöglichkeiten für den Einzelnen ein oder bietet ihm zusätzliche Chancen (Opportunities);

- Teilhabe an Veränderungen des Sozialraums, insbesondere an Entscheidungen der Politik und Verwaltung über die Quartiersentwicklung (Partizipation). Genau solche Partizipation soll Örtliche Teilhabeplanung organisieren und durchsetzen. Wenn das gelingt und die Erkenntnisse der Stadtsoziologie zutreffen, befördert örtliche Teilhabeplanung ganz erheblich die Inklusion in Sozialräumen.

Soziale Inklusion, um die es hier geht, hat also mehrere Dimensionen: eine institutionelle, eine kulturelle, eine politische und eine soziale, darüber hinaus eine ökonomische Dimension ${ }^{7}$. D.h. eine Person kann z.B. aus dem Erwerbssystem ausgeschlossen, ihre kulturelle und soziale Inklusion aber intakt sein. Der heutige Arbeitsmarkt (als Kern der ökonomischen Dimension sozialer Inklusion) ist keine Eigenschaft des Quartiers, sondern ein makrostruktureller Faktor für Inklusion und deshalb für die Sozialraumperspektive eine externe Variable.

Quartiere haben Effekte für die soziale Inklusion, jedoch nur in dem Maße wie die räumliche Nachbarschaft auch die soziale Nachbarschaft ist, d.h. desto mehr sich die sozialen Kontakte auf die nachbarschaftliche Umgebung beschränken, wenn also das Quartier der relevante Sozialraum ist ${ }^{8}$. Die Wirkungen sind somit bei verschiedenen Gruppen der Bewohnerschaft unterschiedlich ausgeprägt. Das hängt auch von individuellen Eigenschaften (Vulnerabilität/Resilienz) ab. Insgesamt sind die Wirkungen gegenüber der sozialen Herkunft und der Einbettung in die Familie nachrangig. Das ist die Quintessenz stadtsoziologischer Forschung zur sozialen Inklusion.

Was heißt das für die Inklusion von Menschen mit Behinderung? Prinzipiell dasselbe wie für alle anderen Menschen in einem Sozialraum auch. Es kommt darauf an, wie sie sich in den einzelnen Sphären der Inklusion bewegen und diese für ihre persönliche Entwicklung nutzen können und welche persönlichen Voraussetzungen und Erfahrun-

6 Häussermann (Fußn. 5), weisen darauf hin, dass auch die Außenwahrnehmung eines Quartiers das Denken und Verhalten der darin lebenden Menschen beeinflusst. Die Stigmatisierung eines Quartiers trägt dazu bei, dass Verantwortung und Bindung an die eigene Lebensumwelt deutlich abnehmen, S. 14-16.

7 Diese analytische Differenzierung von Inklusion wird hier angewendet ohne der Luhmannschen Systemtheorie zu folgen.

8 Dementsprechend handelt es sich bei einem Stadtteil dann um einen Sozialraum, wenn von der sozialen Zusammensetzung dieses Stadtteils Wirkungen auf das Denken und Handeln der Bewohner ausgehen, vgl. Häussermann (Fußn. 5), S. 3. 
gen sie dafür mitbringen. Für Menschen mit Behinderung haben die materielle Infrastruktur und die sozialen Dienste sicher eine relativ größere Bedeutung als für Menschen ohne Behinderung, und bei den sozialen Beziehungen könnte der Aspekt der Unterstützung eine relativ größere Bedeutung haben als bei Menschen ohne Behinderung. Aber auch diese Bedeutungen sind individuell ausgeprägt und abhängig von persönlichen Dispositionen und Interessen.

\section{Barriere Wertschätzung?}

Die Heilpädagogik und Rehabilitationswissenschaft fokussiert im Sozialraum die Menschen ohne Behinderung (ohne diese zu benennen). Aus ihrer Sicht ist ein „kultureller Wandel" erforderlich, wenn inklusive Sozialräume oder Lebensräume auch für Menschen mit Behinderung geschaffen werden sollen. Wesentlicher Inhalt dieses kulturellen Wandels ist die Wertschätzung von Menschen mit Behinderung. Es brauche die „Zuerkennung eines positiven Wertes behinderter Menschen“. Das Fehlen dieser Wertschätzung sei das zentrale Hindernis, um Inklusion im Sozialraum zu verwirklichen. ${ }^{9}$

Aus der Perspektive der dargestellten sozialen Inklusion stellt sich die Frage: warum ist eine besondere Wertschätzung von Menschen mit Behinderung erforderlich, damit inklusive Sozialräume entstehen können? Warum reicht es nicht, dass Menschen mit Behinderungen (mit ihren Eigenarten) genauso akzeptiert werden wie Menschen mit anderen Eigenarten auch?

Der Deutsche Verein hat sich - wie gesagt - nicht dazu geäußert, welche Bedeutung die einzelnen Elemente für die Schaffung eines inklusiven Sozialraums haben. Die drei Zieldimensionen Barrieren, Unterstützungssystem und Diskriminierungsfreiheit/Solidarität stehen gleichberechtigt nebeneinander. Das dritte Ziel thematisiert den Aspekt Wertschätzung, allerdings nicht normativ-ethisch, wie die These oben, sondern pragmatisch, so wie es eine Handlungsstrategie erfordert. Und wie alle anderen Elemente auch, wird dieses Ziel als Aufgabe oder Herausforderung verstanden, nicht als Hindernis. Das ist sozusagen eine normativ-pragmatische Herangehensweise an den inklusiven Sozialraum.

Reduzieren wir den ethischen Anspruch an die Menschen im Sozialraum und übersetzen Wertschätzung mit Akzeptanz oder Anerkennung, dann können wir - einigermaßen - aussagekräftige empirische Forschung befragen. Dazu gehört vor allem die „Berliner Kundenstudie“. Diese ermittelte, dass $80 \%$ der befragten Menschen mit Behinderung ihre Nachbarn als freundlich bezeichnen. ${ }^{10}$ Die Studie zum Ambulant betreuten

9 Diese These hat Dederich auf der Fachtagung vorgetragen, aus der diese Publikation entstanden ist, siehe S. 61 in diesem Band.

10 Seifert, Kundenstudie. Bedarf an Dienstleistungen zur Unterstützung des Wohnens von Menschen mit Behinderung, Berlin 2010, S.129. 
Wohnen in Bayern von Frau Hanslmeier-Prockl 11, die hohe Anforderungen an Nachbarn stellt (,erweitertes Netzwerk“), muss zwar vorsichtig aufgenommen werden, weil die Aussagen zur Nachbarschaft nicht eindeutig sind, aber es lässt sich folgern, dass Akzeptanz im Umgang miteinander ein verbreiteter Umgangsmodus zwischen Menschen mit und ohne Behinderungen ist. Frau Hanslmeier-Prockl stellt fest, dass Reaktionen der Nachbarn eher positiv ausfallen ${ }^{12}$ und „,dass nicht von einer grundsätzlichen Ablehnung von Menschen mit Behinderung gesprochen werden kann ${ }^{13}$ " Vergleicht man diese Ergebnisse mit dem Fazit stadtsoziologischer Forschung, wird deutlich, dass hier ganz „normale“ Nachbarschaften untersucht wurden: „Allein aus lokaler Nähe entsteht nur ein geringes Interesse aneinander - Hauptsache, man stört sich nicht". 14

Von diesen Erkenntnissen lässt sich eine Studie zur „Teilhabe von Menschen mit geistiger Behinderung am Leben in der Kommune" leiten. ${ }^{15}$ Die Autorinnen betonen, dass Menschen mit geistiger Behinderung ein so normales Leben wie möglich führen müssen und das tun sollen, was andere Bürgerinnen und Bürger auch tun: „Angebote machen und Angebote wahrnehmen. Sich selbstverständlich und sichtbar als Helferin beim Stadtfest, als Musiker im Verein und als Kundin beim Bäcker bewegen“, anerkannte und sozial geachtete Rollen ausüben. Dann entstehen „gemeinsame Sozialräume"16. Soziale Inklusion ist nach Ihrer Auffassung am wahrscheinlichsten, wenn Menschen mit Behinderung die materielle Infrastruktur einer Kommune in geeigneter Qualität zugänglich ist und von diesen genutzt wird (insbesondere öffentlicher Personennahverkehr und Institutionen der Bildung und Arbeit) ${ }^{17}$. „Inklusionsbereitschaft" der Bürgerinnen und Bürger ist aus soziologischer Sicht nicht die Voraussetzung dafür, dass Menschen mit Behinderung ebenso Bürgerinnen und Bürger in einem Sozialraum sein oder werden können. Aus pragmatischer Sicht kann darauf verzichtet werden, die Einstellung von Personen mit solchen Kategorien zu kennzeichnen. Viel wichtiger ist es, dass möglichst viele Bewohnerinnen und Bewohner in einem Sozialraum konkrete positive - Erfahrungen im Umgang miteinander machen. Damit entstehen oder verstärken sich Haltungen und Einstellungen, in diesem Fall mit hoher Wahrscheinlichkeit solche, die Inklusion befördern.

Die Studien zur sog. ,gruppenbezogenen Menschenfeindlichkeit“ zeigen, dass Menschen mit Behinderung unter den „Minderheiten“ in Deutschland mit Abstand am meis-

11 Hanslmeier-Prockl, Teilhabe von Menschen mit geistiger Behinderung. Empirische Studie zu Bedingungen der Teilhabe im Ambulant betreuten Wohnen in Bayern, Bad Heilbrunn 2009.

12 Hanslmeier-Prockl (Fußn. 11), S. 93.

13 Hanslmeier-Prockl (Fußn. 11), S. 195.

14 Häussermann/Siebel, Stadtsoziologie. Eine Einführung, Frankfurt/New York 2004, S. 112.

15 Erhadt/Grüber, Teilhabe von Menschen mit geistiger Behinderung am Leben in der Kommune, Freiburg im Breisgau 2011.

16 Erhadt/Grüber (Fußn. 15), S. 125.

17 Erhadt/Grüber (Fußn. 15), S. 58. 
ten akzeptiert werden ${ }^{18}$. Nur etwa sieben Prozent der Befragten stellen den Umfang der Unterstützung und die Ausprägung der Forderungen von Menschen mit Behinderungen in Frage. Es ist schon gewagt, von diesen Umfrageergebnissen darauf zu schließen, dass diese sieben Prozent einen Menschen mit Behinderung als Nachbarn nicht akzeptieren würden, nicht nachvollziehbar ist es, wie von Umfrageergebnissen mit solchen Items auf „Abwertung von Menschen mit Behinderung“ geschlossen werden kann. Mit solchem Herangehen könnten die kommunalen Spitzenverbände in Deutschland verdächtigt werden, Menschen mit Behinderung abwerten zu wollen, weil sie seit Jahren darüber diskutieren, wie die Ausgaben für Menschen mit Behinderung eingegrenzt werden können.

Ich bin ziemlich sicher, dass in einer Soziologie der Inklusion Akzeptanz und Anerkennung grundlegende Kategorien und Konzepte sein müssen und auf eine Ethik der Wertschätzung verzichtet werden kann, um zu verstehen und zu befördern, wie „Menschen mit und ohne Behinderung, alte und junge Menschen, mit und ohne Migrationshintergrund gemeinsam Lebensräume nutzen und gestalten" können - um noch einmal den Deutschen Verein zu zitieren. ${ }^{19}$

Wenn es stimmt, dass ,sich begegnende Personen dazu genötigt sind, auch ihr soziales Gegenüber in einer bestimmten Weise anzuerkennen, um sich in dessen Reaktion selbst anerkannt zu finden - die Anerkennung des Gegenüber wird zur Bedingung des eigenen Anerkannt-Seins“20, dann ergibt sich für die soziale Inklusion von Menschen mit Behinderung tatsächlich eine spezifische Frage: wie ist Reziprozität mit Menschen ohne Behinderung möglich? Ist Reziprozität in solchen zwischenmenschlichen Beziehungen überhaupt möglich?

18 Vgl. zu dazu und zum Folgenden http://www.uni-bielefeld.de/ikg/projekte/GMF/index.htm (Zugriff am 09.08.2012).

19 Eckpunkte des Deutschen Vereins für einen inklusiven Sozialraum, NDV 1/2012, S. 1 ff.

20 Honneth, Verwilderungen. Kampf um Anerkennung im frühen 21. Jahrhundert, Aus Politik und Zeitgeschehen 1-2/2011, S. 37 f. 


\section{Literatur}

Bauer, U./Böcker, M./Rohlwing, S./Dähner, S., Barrierefreie Stadtquartiere, ExWoSt-Sondergutachten im Auftrag des Bundesministeriums für Verkehr, Bau und Stadtentwicklung, Endbericht, Berlin 2011.

Eckpunkte des Deutschen Vereins zur sozialräumlichen Ausgestaltung kommunalen Handelns, NDV 9/2008, S. 377 ff.

Eckpunkte des Deutschen Vereins für einen inklusiven Sozialraum, NDV 1/2012, S. 15 ff.

Erhadt, K./Grüber, K., Teilhabe von Menschen mit geistiger Behinderung am Leben in der Kommune, Freiburg im Breisgau 2011.

Erstes Diskussionspapier des Deutschen Vereins zu inklusiver Bildung, NDV 5/2011, S. 197 ff.

Empfehlungen zur örtlichen Teilhabeplanung für ein inklusives Gemeinwesen, NDV 6/2012, S. $286 \mathrm{ff}$.

Hanslmeier-Prockl, G., Teilhabe von Menschen mit geistiger Behinderung. Empirische Studie zu Bedingungen der Teilhabe im Ambulant betreuten Wohnen in Bayern, Bad Heilbrunn 2009.

Häussermann, H./Schwarze, K./Jaedicke, W./Bär, G./Bugenhagen, I., Möglichkeiten der verbesserten sozialen Inklusion in der Wohnumgebung, Forschungsprojekt im Auftrag des Bundesministeriums für Arbeit und Sozialordnung, Schlussbericht, Berlin 2010.

Häussermann, H./Siebel, W., Stadtsoziologie. Eine Einführung, Frankfurt/New York 2004.

Honneth, A., Verwilderungen. Kampf um Anerkennung im frühen 21. Jahrhundert, Aus Politik und Zeitgeschehen 1-2/2011, S. 37 ff.

Seifert, M., Kundenstudie. Bedarf an Dienstleistungen zur Unterstützung des Wohnens von Menschen mit Behinderung, Berlin 2010. 
III. Inklusion über die Lebensspanne 
1. Alter und Behinderung 


\title{
Behinderung und Pflegebedürftigkeit im Alter - sind die sozialrechtlichen Reaktionen konsistent?
}

\author{
Gerhard Igl
}

I. Zusammenhänge und Zugänge zum Thema:

Behinderung, Pflegebedürftigkeit, Alter

II. Unterschiedliche Reaktionen des Sozialrechts auf die Zusammenhänge von „Alter und Behinderung“ und „Behinderung und Alter" mit Blick auf die Sicherung bei Pflegebedürftigkeit?

1. Unterschiedliche Lebenssituationen: Der alte Mensch mit Behinderung Der behinderte Mensch im Alter

2. Unterschiedliche sozialrechtliche Reaktionen auf diese unterschiedlichen Lebenssituationen?

a) Fragestellungen

b) Nichtökonomische Bedarfe im Alter und bei Behinderung

c) Ökonomischer Bedarf bei Alter und Behinderung

d) Krankheit im Kontext von Alter und Behinderung

e) Pflegebedürftigkeit im Kontext von Alter und Behinderung

f) Behinderung im Alter: Teilhabe und Rehabilitation

g) Die Wirkungen der Wahrnehmung von Alter, Behinderung und Pflegebedürftigkeit in den einzelnen Sozialleistungssystemen

III. Sozialleistungspraxis bei älteren behinderten Menschen

1. Fragestellungen

2. Allgemeine Probleme der Feststellung einer altersspezifischen Leistungspraxis bei Sozialleistungen

3. Zur Problematik der Leistungspraxis der Eingliederungshilfe für ältere behinderte Menschen

a) Fragestellungen

b) Versuch einer Annäherung an die Problematik

IV. Ergebnisse und Schlussfolgerungen

1. Sozialleistungsrecht

2. Sozialleistungsrechtliche Praxis

3. Forschungsbedarf

4. Zur Eingangsfrage 


\section{Zusammenhänge und Zugänge zum Thema:}

\section{Behinderung, Pflegebedürftigkeit, Alter}

Das Thema Behinderung und Pflegebedürftigkeit im Alter und die Frage nach den sozialrechtlichen Reaktionen darauf ist als explizit so formuliertes Thema noch recht jung. ${ }^{1}$ Als implizit formuliertes Thema kursiert es in der sozialpolitischen Diskussion schon seit den sechziger Jahren. Man erinnere sich nur an die Diskussion um die Sicherung im Pflegefall, die sich von Beginn an sehr oft beschränkte auf eine Diskussion um den Schutz pflegebedürftiger älterer Menschen. ${ }^{2}$ Und mit der Bezugnahme auf diese Diskussion sind wir bei einer zentralen Fragestellung unseres Themas, die da lautet, ob sich unsere sozialrechtlichen Sichtweisen auf das Problem Alter und Behinderung oder Behinderung und Alter nicht zu sehr einengen auf den sozialrechtlichen Schutz bei Pflegebedürftigkeit, all dies in dem Wissen, dass die Leistungen bei Pflegebedürftigkeit vor allem von älteren Menschen in Anspruch genommen werden. ${ }^{3}$

Dass das Alter mit Behinderung konnotiert wird, ist üblich. Dass die Behinderung mit dem Alter in Verbindung gebracht wird, erscheint nicht so üblich. Es ist ein Unterschied zu sagen, ich bin ein alter Mensch und im Alter ein behinderter Mensch geworden, oder ich bin als behinderter Mensch alt geworden. Alter und Behinderung wird verstanden als „Alter mit Behinderung“; Behinderung und Alter wird verstanden als „Der behinderte Mensch im Alter“.

Und damit ist man bei einem ersten Unterschied, der sich dann auch sozialrechtlich niederschlagen kann: Alter und Behinderung legen eher nahe, dass die Behinderung altersbedingt ist. Pflegebedürftigkeit im Alter ist eine Form der Behinderung im Alter. Behinderung und Alter hingegen meint, dass die Behinderung schon vor dem Übergang in die Altersphase gegeben war.

Im Folgenden soll untersucht werden, wie das Sozialrecht auf das Alter, wie auf Behinderung und wie auf den Zusammenhang von Alter und Behinderung reagiert. Zum Schluss wird gefragt, ob die sozialrechtlichen Vorkehrungen konsistent sind.

1 Vgl. Welti, Behinderung und Rehabilitation im sozialen Rechtsstaat. Freiheit, Gleichheit und Teilhabe behinderter Menschen. Tübingen 2005; S. 45 ff.; ders. Alter im sozialen Rechtsstaat, BtPrax. 2007, S. 51-55. Igl, Alter und Behinderung im Sozialrecht, in: Berghaus/Bermond/Milz (Hrsg.), Visionen und innovative Konzepte in Zeiten knapper Ressourcen, Kuratorium Deutsche Altershilfe, Köln 2007, S. 12 ff.

2 In der Diskussion um die Einführung eines verbesserten Schutzes bei Pflegebedürftigkeit wurde auch die Festlegung einer Altersgrenze diskutiert, so z.B. Vorschläge der Länder Bayern (BTDrucks. 10/6135) und Rheinland-Pfalz (BR-Drucks. 178/87), s. zur Geschichte die Hinweise bei $I g l$, Pflegeversicherung, in: von Maydell/Ruland/Becker (Hrsg.), Sozialrechtshandbuch (SRH), 5. Aufl. Baden-Baden 2012, S. 929 ff., 932.

3 S. die Angaben in Pflegestatistik 2009, Statistisches Bundesamt, Wiesbaden 2011. 
Die Sozialrechtswissenschaft befasst sich kaum mit Fragen der möglichen besonderen Schutzbelange von älteren Menschen mit Behinderung. ${ }^{4}$ Die Fragen der Schutzbelange des Alters wurden lange Zeit nur unter dem Aspekt der Sicherung der ökonomischen Subsistenz im Rahmen der monetären Alterssicherung diskutiert. Andere Zusammenhänge, auch die Zusammenhänge von Recht und Alter, wurden bisher eher selten angesprochen. ${ }^{5}$ Allerdings steht zu erwarten, dass sich im Zuge der Altersdiskriminierungsdiskussion auch eine Diskussion jenseits der Fragen der Altersdiskriminierung im Arbeitsleben herausbilden wird. ${ }^{6}$

Hingegen werden die rechtlichen Fragen der Schutzbelange von Menschen mit Behinderungen in der Sozialrechtswissenschaft seit einiger Zeit fundiert behandelt. ${ }^{7}$ Mit dem SGB IX hat sich diese Befassung noch verstärkt. Fragen der Eingliederungshilfe für behinderte Menschen nach dem SGB XII sind gerade in jüngerer Zeit Gegenstand rechtlicher, auch rechtspolitischer Befassung. ${ }^{8}$

Vor diesem Hintergrund gesehen befindet sich das zu erörternde Thema „Behinderung und Pflegebedürftigkeit im Alter - sind die sozialrechtlichen Reaktionen konsistent?" in einem Überschneidungsbereich von rechtswissenschaftlicher Artikulation und fast fehlender rechtswissenschaftlicher Befassung.

In dem folgenden Beitrag sollen einige Grundfragen der Herangehensweise an das Thema erörtert werden. Vor allem unter sozialrechtspolitischen Gesichtspunkten soll dies im Abschnitt zu der Frage geschehen, ob das Sozialrecht unterschiedlich auf die Zusammenhänge von „Alter und Behinderung“ und „Behinderung und Alter“ reagiert (Abschnitt II).

Weiter soll einem immer wieder artikulierten Problem der Ausführung der Sozialleistungspraxis nachgegangen werden: Wie sieht es mit der Gewährung von Leistungen der Eingliederungshilfe für ältere Menschen aus? (Abschnitt III). Da zurzeit nicht verlässlich auszumachen ist, ob und in welchem Maße Sozialleistungen die Belange älterer Menschen mit Behinderungen angemessen abdecken, sollen auch Hinweise für den Forschungsbedarf auf diesem Gebiet gegeben werden (Abschnitt IV).

4 Wie Fußn. 1; vgl. auch Igl, Alter und Behinderung im Sozialrecht, in: Hoyer et al. (Hrsg.), Gedächtnisschrift für Jörn Eckert. Baden-Baden, 2008. S. 369-384; ders., Ältere Menschen mit Behinderungen. Realisierung des Teilhabegedankens und Eingliederungshilfe, in: Schütte (Hrsg.), Abschied vom Fürsorgerecht. Von der „Eingliederungshilfe für behinderte Menschen“ zum Recht auf soziale Teilhabe, Berlin 2011, S. 85 ff.

5 Zuletzt Igl/Klie (Hrsg.), Das Recht der älteren Menschen. Baden-Baden 2007; Becker/Roth (Hrsg.), Recht der Älteren, Berlin 2013.

6 S. die umfassende arbeitsrechtliche Analyse von Temming, Altersdiskriminierung im Arbeitsleben. Eine rechtsmethodische Analyse. München, 2008.

7 Welti, Behinderung und Rehabilitation im sozialen Rechtsstaat. Freiheit, Gleichheit und Teilhabe behinderter Menschen, Tübingen 2005.

8 S. den von Schütte herausgegebenen Band und die dortigen Beiträge (wie Fußn. 4). 


\section{Unterschiedliche Reaktionen des Sozialrechts auf die Zusammenhänge von „Alter und Behinderung“ und „Behinderung und Alter" mit Blick auf die Sicherung bei Pflegebedürftigkeit?}

\section{Unterschiedliche Lebenssituationen: Der alte Mensch mit Behinderung - Der behinderte Mensch im Alter}

Kehren wir noch einmal zurück zur Unterscheidung von Alter und Behinderung, verstanden als „Alter mit Behinderung“, und Behinderung und Alter verstanden als „Der behinderte Mensch im Alter".

Alter und Behinderung legen eher nahe, dass die Behinderung altersbedingt ist. Pflegebedürftigkeit im Alter ist eine Form der Behinderung im Alter. Damit ist eine bestimmte Wahrnehmung des älteren Menschen im Sinne einer Defizitwahrnehmung9 verbunden; damit sind auch bestimmte sozialpolitische und sozialrechtliche Reaktionen verbunden. Damit ist vor allem verbunden, dass sich auch in der Bundesrepublik erst spät die Vorstellung durchgesetzt hat, dass Pflegebedürftigkeit ein Phänomen der Behinderung ist. In der sozialrechtlich nicht vorgebildeten Bevölkerung wird man wohl auf Skepsis stoßen, wenn man Pflegebedürftigkeit auch als Behinderung bezeichnet.

Behinderung und Alter hingegen meint, dass die Behinderung schon vor dem Eintritt in die Altersphase gegeben war. Auch damit verbinden sich bestimmte Vorstellungen, werden bestimmte Bilder assoziiert: Die alternde Person mit Down-Syndrom, der ältere körperbehinderte Mensch im Rollstuhl. Dass dieser Personenkreis zusätzlich zur vorhandenen Behinderung im Alter auch noch pflegebedürftig werden kann, wird eher wenig damit assoziiert.

\section{Unterschiedliche sozialrechtliche Reaktionen auf diese unterschiedlichen Lebenssituationen?}

\section{a) Fragestellungen}

Die Frage, ob das Sozialrecht mit den unterschiedlichen Lebenssituationen Alter und Behinderung einerseits und Behinderung und Alter andererseits in unterschiedlicher Weise umgeht bzw. umgehen darf, könnte leicht mit dem Verweis auf den Gleichbehandlungsgrundsatz beantwortet werden. Das Sozialrecht mit seinem ausgeprägten Topos der Gewährleistung von gleicher Behandlung in gleichen Situationen wird den behindert gewordenen alten Menschen nicht anders behandeln können als den alt gewor-

9 Hierzu Vierter Altenbericht, BT-Drucks. 14/8822, S. 65 ff. 
denen behinderten Menschen, es sei denn, es ergäben sich gewichtige Unterschiede, die eine Ungleichbehandlung forderten bzw. rechtfertigten.

Das Verständnis der Problematik für eine mögliche unterschiedliche Herangehensweise des Gesetzgebers an die unterschiedlichen Lebenssituationen eröffnet sich wohl erst in einer historischen Betrachtungsweise der sozialen Sicherungssubsysteme für ältere Menschen und für behinderte Menschen. Diese Sicherungssubsysteme haben sich in sehr unterschiedlichen Zusammenhängen und mit sehr unterschiedlichen Zielsetzungen entwickelt. Zudem stehen diese Sicherungssubsysteme in unterschiedlichen Zusammenhängen. Sie haben sich, wie zu sehen sein wird, jedoch in ihren Zielsetzungen aufeinander zu entwickelt.

\section{b) Nichtökonomische Bedarfe im Alter und bei Behinderung}

Nur eine einzige Sozialleistung in unserem so weit gefächerten Sozialleistungssystem nimmt spezifische nichtökonomische altersbedingte Bedarfe wahr und bezieht sich dabei auf das Alter, nämlich die Altenhilfe im Rahmen der Sozialhilfe (§ 71 SGB XII). Die Altenhilfe umfasst auch Leistungen, die für behinderte ältere Menschen in Betracht kommen können, so die Hilfe bei der Beschaffung und zur Erhaltung einer Wohnung, die den Bedürfnissen des alten Menschen entspricht (§ 71 Abs. 2 Nr. 2 SGB XII), die Beratung und Unterstützung bei der Aufnahme in ein Heim (§ 71 Abs. 2 Nr. 3 SGB XII) und bei der Inanspruchnahme altersgerechter Dienste (§ 71 Abs. 2 Nr. 4 SGB XII). Trotzdem ist zu beachten, dass die Behinderung im Alter leistungsrechtlich nicht explizit thematisiert wird.

\section{c) Ökonomischer Bedarf bei Alter und Behinderung}

Spezielle Sicherungen gerade für das Alter sind vor allem die ökonomischen Sicherungen, d.h. die Einkommenssicherung, die von der Rentenversicherung (SGB VI) oder von der Sozialhilfe in Form der Grundsicherung (Viertes Kapitel des SGB XII) besorgt wird. Bei der Grundsicherung, ebenfalls in der Sozialhilfe geregelt, werden behinderungsbedingte Bedarfe nur im Zusammenhang mit der (Geld-)Leistungshöhe der Grundsicherung berücksichtigt ( $\$ 42$ Satz 1 Nr. 3 iVm $§ 30$ Abs. 1 Nr. 2 SGB XII).

\section{d) Krankheit im Kontext von Alter und Behinderung}

In der Gesetzlichen Krankenversicherung (SGB V) war für lange Zeit kein besonderer Schutz gerade für ältere Menschen vorgesehen. Dies ist auch heute noch so: Zentraler leistungsauslösender Tatbestand der Gesetzlichen Krankenversicherung ist die Krankheit (vgl. § 11 Abs. 1 Nr. 4, § 27 SGB V). 
Seit der Jahrtausendwende zeichnen sich aber Veränderungen in Richtung auf eine sozialleistungsrechtliche Kenntnisnahme von altersspezifischen Gesundheitsbedarfen ab. So stellt die Erweiterung der medizinischen Rehabilitationsmöglichkeiten eine Leistung dar, die de facto insbesondere für ältere Menschen gelten soll ( $\$ 40$ Abs. 1 und 2 SGB V). ${ }^{10}$ Aber auch die anderen ohne Altersbezug in einer der jüngeren Gesundheitsreformen geschaffenen Leistungen der Palliativversorgung 11 und der Erweiterung der häuslichen Krankenpflege auf das betreute Wohnen und andere Wohnformen ${ }^{12}$ präsentieren sich de facto, d.h. in der statistischen Altersverteilung der Leistungsinanspruchnahme, als Leistungen mit Altersbezug. Die Krankenversicherung nähert sich also - im Gesetzestext nicht explizit ausgesprochen - mit ihren Leistungen den gesundheitlichen Bedürfnissen der älteren Menschen.

\section{e) Pflegebedürftigkeit im Kontext von Alter und Behinderung}

Diese Feststellung gilt wiederum in ganz besonderer Weise für die de-facto-Inanspruchnahme von Leistungen der Sozialen Pflegeversicherung (SGB XI). Dieser Sozialversicherungszweig deckt die speziellen Bedarfe ab, die in einer altersspezifischen Behinderungssituation, nämlich der Pflegebedürftigkeit, gegeben sind.

Das Leistungssystem der Sozialen Pflegeversicherung weist bekanntlich ein großes Defizit auf, weil es die Personen mit erheblichem allgemeinem Betreuungsbedarf leistungsrechtlich separiert und damit ein Sonderregime für den - allgemein gesprochen Kreis der Menschen mit Demenz schafft. Immerhin sind in diesem Sonderregime ( $\S 45 a, 45 b$ SGB XI) jetzt auch Personen der sog. Pflegestufe 0 erfasst. Mit der immer noch auf Eis liegenden Erweiterung des Begriffs der Pflegebedürftigkeit könnte hier Abhilfe geschaffen werden. ${ }^{13}$

Erst mit Einführung der Sozialen Pflegeversicherung von 1994 wurde ein Problem des Rehabilitationsgeschehens deutlicher artikuliert: Die Einführung des Vorrangs von Prävention und Rehabilitation vor Pflegeleistungen hat das Bewusstsein für die Rehabilitationsfähigkeit auch des älteren Menschen geöffnet, auch wenn mit dem Vorrangpos-

10 Im Gesetzentwurf zur Gesundheitsreform, BT-Drucks. 16/3100, S. 11, war geplant, Leistungen einzuführen, die gemäß ihrer ursprünglichen Bezeichnung (,geriatrische Rehabilitation“) zum ersten Mal auf eine bestimmte Altersgruppe zugeschnitten sein sollten. Da eine altersgruppenspezifische Rehabilitation jedoch fachlich unangebracht ist, vielmehr die Rehabilitation indikationsspezifisch stattfinden muss, ist in dem zum Gesetz gewordenen Text auf diese Bezeichnung verzichtet worden (vgl. jetzt § 40 SGB V). S. zu dieser Thematik Fuchs, Rechtliche Rahmenbedingungen für die geriatrische Rehabilitation. Was hat sich durch die Gesundheitsreform geändert? Soziale Sicherheit 2007, S. $169 \mathrm{ff}$.

$11 \S 37 \mathrm{~b}$ SGB V, eingeführt durch Art. 1 Nr. 23 des Gesetzes v. 26.3.2007, BGBl. I 2007, S. 378 mit Wirkung vom 1.4.2007.

$12 \S 37$ SGB V, geändert durch Art. 1 Nr. 22 Buchst. a des Gesetzes v. 26.3.2007, BGBl. I 2007, S. 378 mit Wirkung vom 1.4.2007.

13 S. hierzu Wilcken, Pflegebedürftigkeit und Behinderung im Recht der Rehabilitation und Teilhabe und im Recht der Pflege, Berlin 2011 (zugl. Diss. Kiel 2011), S. 300 ff. 
tulat „nur“ die medizinische Rehabilitation gemeint ist (vgl. § 5 SGB XI). Dieses Postulat des Vorrangs von Prävention und medizinischer Rehabilitation blieb mehr als zehn Jahre nach Einführung der Pflegeversicherung meist mehr Ausdruck eines Wunsches als Umsetzung in die Realität. In einer der jüngeren Gesundheitsreformen ${ }^{14}$ musste der Gesetzgeber das gesetzliche Instrumentarium zur Realisierung dieses Rehabilitationsanliegens nach mehreren Versuchen noch einmal schärfen, damit die Sozialleistungsträger den gesetzlichen Auftrag als verbindlichen Auftrag begreifen und damit nicht nur der Vorrang der Prävention und Rehabilitation, sondern auch der Vorrang des Gesetzes, d.h. die Beachtung des Gesetzes durch die Sozialleistungsträger, hier endlich Realität wird.

Vielleicht haben die Schwierigkeiten bei der Realisierung des Postulates „Rehabilitation vor Pflege" auch damit zu tun, dass es im deutschen Rehabilitationswesen lange Zeit unüblich war, ältere Menschen zu rehabilitieren, ohne dass es um die Wiedereingliederung in den Arbeitsmarkt ging. Dazu gleich noch einmal:

\section{f) Behinderung im Alter: Teilhabe und Rehabilitation}

Der sozialrechtliche Schutz behinderter Menschen hat mit dem SGB IX explizit die Zielsetzung der Förderung der gleichberechtigten Teilhabe am Leben in der Gesellschaft erhalten (§ 1 Satz 1 SGB IX). Dieser Zielsetzung dienen die Leistungen zur Teilhabe ( $\$ 4$ Abs. 1 SGB IX). Diese moderne Rahmensetzung für den sozialen Schutz behinderter Menschen lässt leicht in Vergessenheit geraten, dass das deutsche Sozialleistungssystem seine Teilhabebemühungen lange Zeit vornehmlich auf die (Wieder)Erlangung von Erwerbsfähigkeit konzentriert hat. Dem alten und behinderten Mensch hat man trotz eines ausgebauten Rehabilitationswesen keine besonderen Rehabilitationsbemühungen angedeihen lassen. Der alte Mensch hat als nicht mehr rehabilitationsfähig gegolten. Was eine solche Wahrnehmung in der Sache ausmacht, wird erst dann besonders deutlich, wenn man dies in die heutige Terminologie übersetzt und nicht sagt, der alte Mensch ist nicht mehr rehabilitationsfähig, sondern, der alte Mensch ist nicht mehr teilhabefähig. Freilich gab es auch schon vor dem SGB IX von 2001 Rehabilitationsleistungen, häufig betitelt als Leistungen zur sozialen Rehabilitation, denen die in $\S 4$ Abs. 1 Nr. 4 SGB IX formulierte Zielsetzung der gesellschaftlichen Teilhabe innewohnte, so in der Eingliederungshilfe des Sozialhilferechts.

Die Eingliederungshilfe des Sozialhilferechts verfügte schon im Bundessozialhilfegesetz (BSHG) über die breiteste Leistungsspreizung für behinderte Personen (vgl. $\S \S 53,54$ SGB XII) und wurde deshalb der allgemeinen Teilhabezielsetzung am ehesten gerecht. Das höhere Lebensalter war, abgesehen von den schulischen Leistungen, ge-

14 Hierzu Igl, Das Gesetz zur strukturellen Weiterentwicklung der Pflegeversicherung, NJW 2008, S. $2214 \mathrm{ff}$. 
setzlich nicht als Ausschlusskriterium von bestimmten Leistungen vorgesehen. ${ }^{15}$ Aber ein Blick in die Eingliederungshilfe-Verordnung ${ }^{16}$ zeigt, dass sich das Leistungsspektrum auf die schulische und berufliche Ausbildung und die Eingliederung in das Arbeitsleben konzentriert. Spezielle Teilhabeleistungen für ältere Menschen sieht die Eingliederungshilfe-Verordnung nicht vor.

\section{g) Die Wirkungen der Wahrnehmung von Alter, Behinderung und \\ Pflegebedürftigkeit in den einzelnen Sozialleistungssystemen}

Das Sozialleistungssystem reagiert erst in jüngerer Zeit auf die besonderen Belange im Zusammenhang von Alter und Behinderung, allerdings nicht auf eine Weise, in der sich der Zusammenhang von Alter und Behinderung leistungsrechtlich explizit abbilden würde. Die Leistungsbereiche, in denen gerade der Zusammenhang von Alter und Behinderung eine Rolle spielt, nennen diesen Zusammenhang nicht. Dies gilt für die Leistungen bei Pflegebedürftigkeit. Diese Leistungen kommen statistisch ganz überwiegend hochaltrigen Personen zugute. Der Zusammenhang von Alter und Behinderung versteckt sich, so gesehen, leistungsrechtlich hinter dem Begriff der Pflegebedürftigkeit.

Die Wahrnehmung von älteren Menschen als medizinisch rehabilitationsfähigen Personen ist ebenfalls der Pflegeversicherung zu verdanken. Der gesetzlich angeordnete Vorrang von Prävention und medizinischer Rehabilitation vor Pflege hat hierfür das Verständnis eröffnet.

Auch in der Gesetzlichen Krankenversicherung sind in der letzten Dekade Leistungen aufgenommen worden, die - wiederum ohne es zu benennen - vor allem älteren Menschen zugutekommen sollen.

Der Zusammenhang von Alter und Behinderung bildet sich somit sozialleistungsrechtlich in drei Dimensionen ab: in der Dimension des Schutzes bei Pflegebedürftigkeit, in der Dimension der medizinischen Rehabilitationsfähigkeit und in der Dimension spezieller Gesundheitsleistungen. Allen drei Dimensionen liegt eine Form der Behinderung zugrunde, und allen drei Dimensionen ist gemeinsam, dass die Leistungen de facto, das heißt in der statistischen Verteilung, für Menschen höheren Alters besonders einschlägig sind.

Diese Reaktionen auf den Zusammenhang von Alter und Behinderung zeigen zweierlei: Erstens steht im Vordergrund des Sozialleistungsrechts der ältere Mensch, der im Alter behindert in Form der Pflegebedürftigkeit geworden ist, nicht der behinderte Mensch, der älter geworden ist. Zweitens wird das Alter nicht zum leistungsauslösenden Kriterium erhoben, was im Übrigen dem Gleichbehandlungsgrundsatz sehr entspricht.

15 Das wird auch in der Rechtsprechung immer wieder betont, s. zuletzt den Beschluss des LSG Baden-Württemberg vom 19.03.2012, Az.: L 2 SO 72/12 ER-B, Rdnr. 10, zitiert nach juris.de. S. auch BVerwG v. 21.12.2005, Az.: 5 C 26/04, Rdnr. 13, zitiert nach juris.de = NVwZ-RR 2006, 406.

16 Verordnung nach $\S 60$ SGB XII (Eingliederungshilfe-Verordnung). 
Damit bleiben vor allem die Leistungen der Eingliederungshilfe des SGB XII für jene Personen bestimmt, die als behinderte Menschen alt geworden sind. Im SGB XII sind das vor allem die Leistungen, die nicht eine schulische oder berufliche Rehabilitation zum Gegenstand haben.

Der Ausgangspunkt für das Sozialleistungsrecht ist für den behinderten Menschen im Alter ein anderer als für den im Alter behindert, d.h. pflegebedürftig gewordenen Menschen. Der behinderte Mensch erhält, bevor er älter wird, Leistungen, die ihm die Teilhabe in den verschiedenen Lebensbereichen sichern sollen (vgl. § 4 Abs. 1 SGB IX). Diese Teilhabeleistungen, die im Lebensabschnitt des Erwerbstätigenalters vor allem Leistungen zur Teilhabe am Arbeitsleben sind, stellen in diesem Lebensabschnitt das Zentrum des Rehabilitationsgeschehens dar. Alle anderen Leistungen zur Teilhabe gruppieren sich um die Teilhabe am Arbeitsleben, sie stehen aber nicht im Zentrum. Wenn dieses Zentrum bei Erreichen der Rentenaltersgrenze wegfällt, kommt dann den anderen Teilhabeleistungen mehr Gewicht zu.

\section{Sozialleistungspraxis bei älteren behinderten Menschen}

\section{Fragestellungen}

Im Folgenden soll nun einem Problem nachgegangen werden, das mit einem mancherorts ausgesprochenem Unbehagen zu tun hat, nämlich der Frage, ob gerade bei behinderten Menschen ab einer gewissen Schwelle des höheren Alters Leistungen der Eingliederungshilfe nicht mehr gewährt werden, und ob diese Personen ,in die Pflegeversicherung abgeschoben“ werden. Das würde, etwas verkürzt gesagt, bedeuten, dass diesem Personenkreis Leistungen zur Teilhabe am Leben in der Gemeinschaft aus Altersgründen versagt würden. ${ }^{17}$ Wie es dann mit dem Personenkreis aussieht, der erst im Alter behindert, d.h. pflegebedürftig geworden ist, und der dann ebenfalls zusätzlich auf Teilhabeleistungen angewiesen ist, wäre dann die andere Seite der Betrachtung der Realisierung des Teilhabegedankens bei älteren Menschen mit Behinderung.

Bei der Erörterung dieser Fragen kann man es möglicherweise auch mit einer spezifischen Diskriminierungsproblematik gegenüber älteren Menschen beim Sozialleistungsbezug zu tun haben. Deshalb zunächst einige Worte zu diesem Thema.

17 S. hierzu auch den Beitrag von Welti, Alter im sozialen Rechtsstaat, BtPrax. 2007, S. 51 ff. 


\section{Allgemeine Probleme der Feststellung einer altersspezifischen Leistungspraxis} bei Sozialleistungen

In verschiedenen Zusammenhängen wird eine altersspezifische Leistungspraxis bei Sozialleistungen gerügt. ${ }^{18} \mathrm{Zu}$ unterscheiden ist dabei zwischen einer altersspezifischen Leistungszuweisung (Leistungszugang) und einer altersspezifischen Art und Weise der Leistungserbringung.

In die erste Kategorie fallen dabei die rationierte Zuteilung bzw. die Nichtgewährung von bestimmten Sozialleistungen an ältere Menschen. Die Debatte hierüber kann an dieser Stelle nicht aufgenommen werden. Sie betrifft vor allem bestimmte Gesundheitssozialleistungen für Menschen ab einem definierten höheren Lebensalter. In diese erste Kategorie (Leistungszugang) fällt auch eine spezifische Praxis von Sozialleistungsträgern, Leistungen für ältere Menschen nicht mehr zu gewähren, obwohl die Leistungsvoraussetzungen hierfür noch gegeben sind. Dies gilt für die Nicht- und Nichtweitergewährung von Leistungen der Eingliederungshilfe für ältere Menschen auf dem Gebiet der Sozialhilfe. Diesem Thema soll im Folgenden nachgegangen werden.

Die zweite Kategorie einer altersspezifischen Leistungspraxis betrifft die Art und Weise der Leistungserbringung, z.B. den Umfang und die Intensität einer medizinischen Behandlung bei einem älteren Menschen im Vergleich zu einem Menschen im jüngeren Alter. Im Zusammenhang dieser Kategorie wird häufig eine Bewertung vorgebracht im Sinne einer besseren/schlechteren Behandlung. Allerdings wird manchmal nicht gesehen, dass eine andere Art und Weise der Behandlung im Alter unter Umständen fachlich geboten sein kann. So sieht beispielsweise die Psychiatrie-Personalverordnung 19 in der Personalbemessung in der Gerontopsychiatrie im Vergleich zur allgemeinen Psychiatrie geringere Minutenwerte für den Einsatz des ärztlichen Personals vor ( 5 Abs. 1 PsychPV). Daraus kann aber nicht gefolgert werden, dass der Kreis der gerontopsychiatrischen Patientinnen und Patienten medizinisch schlechter versorgt wird als derjenige der allgemeinen Psychiatrie. Vielmehr ist, spezifisch an den Therapiebedarfen der gerontopsychiatrischen Patientinnen und Patienten orientiert, sogar ein verstärkter Einsatz von Krankenpflegepersonal und Bewegungstherapeuten, Krankengymnasten und Physiotherapeuten vorgesehen (§ 5 Abs. 1 Psych-PV).

18 Die Angaben oder Hinweise sind jedoch zum Teil wenig verlässlich. An umfassenden und belastbaren repräsentativen Untersuchungen fehlt es bislang.

19 Verordnung über Maßstäbe und Grundsätze für den Personalbedarf in der stationären Psychiatrie (Psychiatrie-Personalverordnung - Psych-PV). 
Behinderung und Pflegebedürftigkeit im Alter - sind die sozialrechtlichen Reaktionen konsistent?

\section{Zur Problematik der Leistungspraxis der Eingliederungshilfe für ältere} behinderte Menschen

\section{a) Fragestellungen}

Ein besonderes Problem stellt die teilweise vermutete Praxis der Sozialleistungsträger bei der Vergabe von bestimmten Leistungen der Eingliederungshilfe für ältere Menschen dar, dies auch an der Schnittstelle zu den Leistungen der Pflegeversicherung. Hier wird teilweise - allerdings nicht belastbar - vorgebracht, dass sich gewisse Praktiken der Leistungsverlagerung bzw. der Leistungsversagung vor allem im Altersbereich zwischen 60 und 65 Jahren ergeben haben.

Den weiteren Ausführungen ist vorwegzuschicken, dass in der Bundesrepublik keine repräsentative oder gar flächendeckende Untersuchung zur Leistungspraxis der Sozialhilfeträger existiert. Verwaltungsinterne Anweisungen, Verwaltungsvorschriften in den verschiedenen rechtlichen Ausprägungen und andere untergesetzliche Vorschriften sind oft nicht publiziert oder zugänglich. Die Sozialhilferichtlinien der Länder sind nur in wenigen Fällen im Internet recherchierbar. In den großen juristischen Datenbanken sind sie - soweit ersichtlich - nicht enthalten.

Hier ist bereits Forschungsbedarf angemahnt worden. ${ }^{20}$ Bis jetzt liegen allerdings keine einschlägigen Untersuchungen vor. Der Sechste Altenbericht ${ }^{21}$, vorgelegt im Juni 2010, enthält ein Kapitel zu Altersgrenzen bei Sozialleistungen im Bereich Gesundheit, Pflege und Eingliederung. Leider hat es die Altenberichtskommission versäumt, hier einschlägige Gutachten einzuholen, damit endlich mit belastbaren Daten gearbeitet werden kann. Die Bundesregierung ist in ihrer Stellungnahme zum 6. Altenbericht nicht auf diese Problematik eingegangen. ${ }^{22}$

\section{b) Versuch einer Annäherung an die Problematik}

Man kann die geschilderte vermutete Leistungspraxis der Eingliederungshilfe für ältere behinderte Menschen unter dem Aspekt der Rationierung von bestimmten Sozialleistungen betrachten. Die Diskussion um die Rationierung bestimmter Leistungen aus Altersgründen findet in der Bundesrepublik mittlerweile breit statt. Sie konzentriert und beschränkt sich allerdings auf die Thematik der Rationierung von Gesundheitsleistungen. ${ }^{23}$ Die dort gebräuchliche Unterscheidung von impliziter und expliziter Rationie-

$20 \mathrm{Igl}$, in: Schütte (Fußn. 4), S. 95 f.

21 BT-Drucks. 17/3815, Sechster Bericht zur Lage der älteren Generation in der Bundesrepublik Deutschland. Altersbilder in der Gesellschaft und Stellungnahme der Bundesregierung, S. $206 \mathrm{f}$.

22 Igl, in: Schütte (Fußn. 4), S. I ff.

23 So auch die entsprechende Expertise für den 6. Altenbericht (Fußn. 21) von Remmers, Rationierung und Altersdiskriminierung, in: Berner/Rossow/Schwitzer (Hrsg.), Altersbilder in der Wirtschaft, im 
rung 24 und die Unterscheidung nach den Wirkungsebenen (Mikro-, Meso- und Makroebene) $)^{25}$ kann für die hier vorliegende Problematik zumindest begrenzt weiter helfen.

Versteht man unter Rationierung die Zuteilung begrenzt vorhandener Güter nach bestimmten Kriterien, dann wären die begrenzt vorhandenen Güter hier bestimmte Leistungen der Eingliederungshilfe, die dem Buchstaben und Sinn des Gesetzes entsprechend nur gemäß den gesetzlich beschriebenen Kriterien, nicht aber gemäß implizit angebrachten Kriterien wie Alter und/oder Pflegebedürftigkeit zugeteilt werden dürften. Diese implizite Rationierung würde dann auf der Mikroebene der Sozialleistungsgewährung, also im Verhältnis zwischen Sozialhilfeträger und Sozialhilfeberechtigtem, stattfinden. Wenn Sozialhilfeträger ein solches Verhalten gesteuert, z.B. durch entsprechende Richtlinien veranlasst, pflegen, würde die implizite Rationierung auf der Mesoebene organisiert werden. Dass eine solche implizite Rationierung in Widerspruch zum Gesetz steht, bedarf keiner näheren Erläuterung.

Die Besonderheit einer solchen Rationierung kann aber darin gesehen werden, dass die Sozialhilfeträger aus ihrer Sicht die betroffenen Personen nicht ohne Leistung lassen, sondern diese im Leistungssystem der Sozialen Pflegeversicherung als gut aufgehoben und versorgt betrachten. Solche Erwägungen - auch hier kann man nur aus eigenen Wahrnehmungen berichten - sind Sozialhilfeträgern nicht fremd. Schon bei Einführung der Pflegeversicherung ist bekannt geworden, dass es viele Bemühungen seitens der Sozialhilfeträger gegeben hat, behinderte Menschen in die Pflegeversicherung ,abzuschieben“. Vor diesem Hintergrund erlangt § 43a SGB XI - Reduzierung der Pflegeleistungen für Pflegebedürftige in vollstationären Einrichtungen der Hilfe für behinderte Menschen - eine besondere Konnotation.

Bei meinen eigenen statistischen Analysen zum Thema der Leistungspraxis der Eingliederungshilfe ${ }^{26}$ konnte ich immerhin zwei Ergebnisse festhalten:

- Der einwohnerproportionale Anteil der Empfänger von Leistungen der Eingliederungshilfe im Laufe des Berichtsjahres 2008 variiert zwischen den Bundesländern erheblich. ${ }^{27}$

- Mit zunehmendem Alter der Leistungsempfänger steigen die Anteile der nicht altersspezifischen Leistungen der Eingliederungshilfe von Alterskohorte zu Alterskohorte an. Lediglich die Hilfen zur Förderung der Verständigung mit der Umwelt bleiben anteilig fast auf gleicher Höhe, während der Anteil der anderen Leistungen zur Teilhabe am Leben in der Gemeinschaft fast um die Hälfte abnimmt. Im Verhältnis zu allen Beziehern von Leistungen der Eingliederungshilfe stellt

Gesundheitswesen und in der pflegerischen Versorgung. Expertisen zum Sechsten Altenbericht der Bundesregierung, Band 2, Wiesbaden 2012, S. 339-368.

$24 I g l$, in: Schütte (Fußn. 4), S. 343 f.

25 Igl, in: Schütte (Fußn. 4), S. 345 f.

26 Igl, in: Schütte (Fußn. 4), S. 92 ff.

27 Mittelwert Bundesrepublik: 8,7; höchster Wert in Mecklenburg-Vorpommern mit 13,0; niedrigste Werte in Baden-Württemberg $(6,0)$ und Bremen $(5,9)$, jeweils bezogen auf 1000 Einwohner; Statistisches Bundesamt, 2010, Tabelle B. 6.2. 
sich der Anteil der nicht altersspezifischen Leistungen bei den Hilfen zum selbstbestimmten Wohnen und bei den Leistungen zur Teilhabe am Leben in der Gemeinschaft in den jeweiligen Alterskohorten als ansteigend dar.

Mit diesen Ergebnissen ist sicher noch nicht viel gewonnen. Sie zeigen aber, dass ein pauschalierender Rationierungsvorwurf gegenwärtig mangels belastbaren Datenmaterials spekulativ bleibt. Nochmals ist also Forschungsbedarf anzumelden.

\section{Ergebnisse und Schlussfolgerungen}

Die Realisierung des Teilhabegedankens für ältere Menschen mit Behinderung hängt nicht nur von der sozialleistungsrechtlichen Situation, sondern auch von der Praxis der Sozialleistungsgewährung ab.

\section{Sozialleistungsrecht}

Das Sozialleistungsrecht bildet die besonderen Schutzbelange im Zusammenhang von Alter und Behinderung nicht explizit und nicht in allgemeiner Weise ab. Der Zusammenhang von Alter und Behinderung bildet sich sozialleistungsrechtlich aber in drei speziellen Dimensionen ab: in der Dimension des Schutzes bei Pflegebedürftigkeit, in der Dimension der medizinischen Rehabilitationsfähigkeit und in der Dimension spezieller Gesundheitsleistungen. Allen drei Dimensionen liegt eine Form der Behinderung zugrunde, und allen drei Dimensionen ist gemeinsam, dass die Leistungen de facto, das heißt in der statistischen Verteilung, für Angehörige der höheren Altersgruppen besonders einschlägig sind. Das Sozialleistungsrecht unterscheidet auch nicht danach, ob ein älterer Mensch im Alter behindert wird, oder ob ein behinderter Mensch älter wird.

Die Frage, ob die sozialleistungsrechtlichen Vorkehrungen in besonderer Weise den Belangen älterer Menschen mit Behinderungen, auch in Form von Pflegebedürftigkeit, Rechnung tragen sollten, muss dann mit Nein beantwortet werden, wenn mit den vorhandenen Leistungen bereits diesen Belangen Rechnung getragen wird. Wenn dies der Fall ist, bedarf es keiner eigenen Kategorie von Leistungen für ältere Menschen mit Behinderungen. Alleine die Tatsache, dass bestimmte Sozialleistungen statistisch von Angehörigen der älteren Generation in höherem Maß als von Angehörigen jüngerer Generationen in Anspruch genommen werden, spricht noch nicht dafür, dass solche besonderen Kategorien von Leistungen explizit, d.h. nur für ältere Menschen mit Behinderungen, geschaffen werden sollten. Dies hätte für andere, situativ gleich betroffene Personen Nachteile, denn jede gesetzlich angebrachte Altersgrenze wirkt für Personen jenseits des anspruchsbegründenden Alters leistungsausschließend. Solange gewährleistet ist, dass das vorhandene Sozialleistungsspektrum die Belange älterer Menschen mit 
Behinderungen abdeckt, ist daher von besonderen Leistungen für diesen Personenkreis abzusehen. Das setzt aber voraus, dass in der Leistungspraxis das Leistungsspektrum ausgeschöpft wird.

\section{Sozialleistungsrechtliche Praxis}

In der sozialleistungsrechtlichen Praxis werden Andeutungen gemacht und Vermutungen geäußert, dass die Gewährung von Leistungen der Eingliederungshilfe des SGB XII an ältere Menschen mit Behinderungen eingeschränkt wird, u.a. auch mit dem Hinweis auf mögliche Leistungen der Pflegeversicherung (SGB XI). Anhand des vorliegenden statistischen Materials sind diese Annahmen nicht belegbar. Die vorliegenden Daten deuten sogar darauf hin, dass ältere Menschen mit Behinderungen bestimmte Teilhabeleistungen der Eingliederungshilfe mit höherem Anteil erhalten als jüngere Menschen mit Behinderungen. Sozialwissenschaftliche fundierte und belastbare Daten und Erkenntnisse liegen jedoch nicht vor.

\section{Forschungsbedarf}

Angesichts der unbefriedigenden Erkenntnisse über die Praxis der Gewährung von Teilhabeleistungen für ältere Menschen mit Behinderungen ist es angezeigt, hier entsprechende Untersuchungen vorzunehmen. Diese Untersuchungen sollten in zwei Richtungen gehen:

- Feststellung des Anteils der Inanspruchnahme der einzelnen Hilfen im Rahmen der Eingliederungshilfe nach dem SGB XII durch ältere Menschen mit Behinderungen im Vergleich zu jüngeren Menschen mit Behinderung (Bundes-, Länderund Sozialhilfeträgervergleich);

- Untersuchung der Frage nach einer Praxis von Sozialhilfeträgern, ob ab einer bestimmten Altersgrenze bestimmte Leistungen der Eingliederungshilfe versagt und ggf. auch durch Leistungen der Pflegeversicherung abgelöst werden.

Diese Untersuchungen könnten auch unter dem Gesichtspunkt durchgeführt werden, ob die Teilhabebelange älterer Menschen mit Behinderungen mit dem vorhandenen Sozialleistungsspektrum angemessen abgedeckt sind und ob ggf. ein Bedarf an weiteren Leistungen oder an Veränderungen bzw. Präzisierungen vorhandener Leistungen besteht. $^{28}$

28 Für seelisch behinderte Kinder und Jugendliche ist ein solcher besonderer Schutz bereits realisiert, vgl. § 35a SGB VIII. Hierzu Greß/Rixen/Wasem, Eingliederungshilfe für seelisch behinderte Kinder und Jugendliche: Abgrenzungsprobleme und Reformszenarien, Vierteljahresschrift für Sozialrecht 2009, S. $43 \mathrm{ff}$. 
Mit diesen Untersuchungen könnte auch ein Beitrag zu der Forderung der 85. ASMK ${ }^{29}$ nach einer personenzentrierten teilhabeorientierten Weiterentwicklung des Eingliederungshilfesystems geleistet werden.

\section{Zur Eingangsfrage}

Auf die in der Überschrift gestellte Frage ,Sind die sozialrechtlichen Reaktionen auf Behinderung und Pflegebedürftigkeit im Alter konsistent?" kann mit zwei Sätzen im Konjunktiv geantwortet werden:

Die sozialrechtlichen Reaktionen wären konsistent,

- wenn in der Praxis der Eingliederungshilfe das vorhandene Leistungsspektrum auch für ältere behinderte und pflegebedürftige Menschen ausgeschöpft würde, und

- wenn der Begriff der Pflegebedürftigkeit so gefasst und gesetzlich implementiert würde, dass die Teilhabe auch der Menschen mit allgemeinem Betreuungsbedarf gesichert ist.

Der Antwort auf die in der Überschrift gestellte Frage ist hinzuzufügen, dass Pflegebedürftigkeit als Ausdruck einer Behinderung zu sehen ist. Damit würden die Leitprinzipien des SGB IX auch für pflegebedürftige Menschen gelten. Damit würde auch die Selbstverständlichkeit der Geltung dieser Leitprinzipien bei der Aufgabenwahrnehmung der Sozialhilfeträger in Richtung auf behinderte ältere Menschen unterstrichen werden, die heute noch als pflegebedürftig, nicht aber als behindert angesehen werden.

29 85. Arbeits- und Sozialministerkonferenz 2008, Weiterentwicklung der Eingliederungshilfe für Menschen mit Behinderungen. Hamburg, 13./14. November 2008. 


\title{
Behindertenrecht und Behindertenpolitik in der Kommune - ein Blick in die Rechtsprechung
}

\author{
Stephan Rittweger
}

$\begin{array}{ll}\text { I. Einleitung } & 135\end{array}$

II. Entwicklungen in der neueren Rechtsprechung 136

1. Kein Auto zulasten der Sozialhilfe 136

2. § 71 SGB XII: Kulturelle Teilhabe, kein Anspruch auf Theaterbesuch 136

3. Kein Sport-Rollstuhl - Krankenversicherung 137

4. Inklusionsziel Bildung 138

5. Kein Cialis zulasten der Gesetzlichen Krankenversicherung 139

III. Zusammenfassung und Ausblick 139

\section{Einleitung}

Die Gerichte haben als Dritte Gewalt im Staate die klassische Aufgabe, die anderen Staatsgewalten zu kontrollieren. Die Sozialgerichte haben den speziellen Auftrag, die Sozialleistungsträger zu überprüfen und sie immer wieder anzuhalten, dass jedem Berechtigten die ihm zustehenden Sozialleistungen in zeitgemäßer Weise, umfassend und zügig zukommen vgl. § 17 SGB I. Dabei hat das Bundesverfassungsgericht die Sozialgerichte schon manches Mal daran erinnert, dass sie sich schützend und fördernd vor die Rechtsgüter der Betroffenen stellen sollen. Diese Aufgabenstellung erfordert, Gerichtsverfahren einem zeitnahen Ende durch gütliche Einigung zuzuführen. Klagen über mehrere Instanzen und Jahre hinweg schaden oft mehr als sie den Betroffenen nutzen. Die weit überwiegende Zahl der Verfahren vor den Sozialgerichten endet deshalb unstreitig, Anerkenntnisse und Vergleiche beenden weitaus mehr Verfahren als Urteile und Beschlüsse. Das ist der Grund, warum ein Überblick über die sozialgerichtliche Rechtsprechung keineswegs vollständig abbildet, wie Sozialbehörden und Sozialgerichte zur Inklusion stehen.

Die Aufgaben der sozialgerichtlichen Rechtsprechung erschöpfen sich aber nicht in der Schaffung von Rechtsfrieden im Einzelfall. An vielen Stellen des Sozialgesetzbuches hat der Gesetzgeber unbestimmte Rechtsbegriffe verwendet, ja verwenden müssen. Deren Inhalte zu bestimmen ist eine weitere bedeutsame Aufgabe der Gerichte. Ihre 
Entscheidungen konkretisieren die Regelungen der Gesetze; auch bilden sie das Recht fort. Hier haben die Sozialgerichte einen Definitionsauftrag und eine Definitionshoheit.

Dies vorausgeschickt ist festzustellen, dass die Rechtsprechung bereit ist, sich auf die UN-Behindertenrechtskonvention als normative Handlungsdirektive einzustellen. Die Sozialgerichte lassen nach und nach erkennen, welche konkreten Rechtsansprüche aus dem abstrakten Ziel der Inklusion folgen - und wie die Balance zwischen Inklusion und Finanzlast zu finden ist.

\section{Entwicklungen in der neueren Rechtsprechung}

\section{Kein Auto zulasten der Sozialhilfe}

In einem Fall, über den das Bayerische LSG zu entscheiden hatte, machte ein 53 Jahre alter Kläger (GdB 100, Merkzeichen G, B, RF und aG) beim Träger der Sozialhilfe geltend, er benötige besondere Hilfen, um kulturelle Veranstaltungen besuchen zu können. Dazu sei er wegen seiner Wohnlage und seiner Behinderung auf einen PKW angewiesen. Er verlangte daher Kostenerstattung für Reparatur, TÜV, Versicherung und Benzin nach $\S \S 53,54$ SGB XI iVm der Eingliederungshilfe-VO.

Mit Urteil vom 26.02.2010 ${ }^{1}$ erkannte das Gericht einen Anspruch des Klägers auf Teilhabe an kulturellen Veranstaltungen dem Grunde nach an. Die KFZ-Kosten seien aber nicht zu übernehmen, denn der Behindertenfahrdienst des Bezirks ermögliche es dem Kläger in noch ausreichendem Umfang, am Leben in der Gemeinschaft teilzunehmen. Der Kläger könne auf diesem Wege einmal monatlich kulturelle Veranstaltungen besuchen und soziale Kontakte pflegen. Auch sei die Inanspruchnahme von öffentlichen Verkehrsmitteln mithilfe der Ehefrau zumutbar (§ 2 Abs. 1 SGB XII).

Die UN-Behindertenrechtskonvention wird in dieser Entscheidung nicht benannt.

\section{2. § 71 SGB XII: Kulturelle Teilhabe, kein Anspruch auf Theaterbesuch}

In einem weiteren Fall ging es um einen 1938 geborenen Kläger, der Grundsicherung im Alter bezog. Er bat um Auskunft, welche Möglichkeiten die Altenhilfe nach dem SGB XII eröffne, ihn zu unterstützen. Der Gesetzgeber habe bei § 71 SGB XII an eine finanzielle monatliche Unterstützung zum Besuch von Veranstaltungen oder Einrichtungen gedacht. Auch die Gewährung von Reisebeihilfen bis hin zu einem Erholungsurlaub sei in $\S 71$ SGB XII enthalten.

1 Az. L 8 SO 55/09. 
Der achte Senat des Bayerischen LSG hat mit Urteil vom 26.02.2010² einen Anspruch auf Teilhabe am kulturellen Leben dem Grunde nach anerkannt, im konkreten Fall aber für den Kläger verneint. Zu den persönlichen Bedürfnissen des täglichen Lebens gehörten im Rahmen der Altenhilfe gem. § 71 SGB XII in vertretbarem Umfang auch Beziehungen zur Umwelt und eine Teilnahme am kulturellen Leben (§ 27 Abs. 1 S. 2 SGB XII). Die Leistungen der Altenhilfe seien in einem Katalog nach § 71 Abs. 2 Nr. 5 SGB XII konkretisierend derart beschrieben, dass insbesondere Leistungen zum Besuch von Veranstaltungen oder Einrichtungen, die der Geselligkeit, der Unterhaltung, der Bildung oder den kulturellen Bedürfnissen alter Menschen dienen, in Betracht kämen. Damit komme durchaus ein individueller Einzelanspruch in Betracht. Voraussetzung für derartige Ansprüche sei aber das konkrete Vorliegen einer entsprechenden Infrastruktur und eines entsprechenden Angebots sowie deren Geltendmachung. Insoweit fehle es dem Kläger aber zur unmittelbaren Geltendmachung von Ansprüchen an einem Rechtsschutzbedürfnis, weil ihm keine konkreten Vergünstigungen versagt worden seien.

Zwar seien die Bedarfe abweichend von $\S 28$ Abs. 1 S. 2 SGB XII festzulegen, wenn im Einzelfall ein Bedarf ganz oder teilweise anderweitig gedeckt sei oder unabweisbar sei und der Höhe nach erheblich von einem durchschnittlichen Bedarf abweiche. Es sei aber nicht ersichtlich, wie hier der Bedarf des Klägers unabweisbar seiner Höhe nach erheblich vom üblichen Bedarf abweiche. Dieser könne jedenfalls nicht dadurch begründet werden, dass bestimmte kulturelle Bedürfnisse des Klägers aufgrund seiner bisherigen Lebensführung besonders ausgeprägt vorhanden seien.

Die UN-Behindertenrechtskonvention wird auch in dieser Entscheidung nicht benannt.

\section{Kein Sport-Rollstuhl-Krankenversicherung}

In einem vom BSG mit Urteil vom 18.05.2011 ${ }^{3}$ entschiedenen Fall begehrte der Kläger einen Sportrollstuhl, um am Behinderten-Basketball im Verein teilnehmen zu können. Der Rollstuhl, mit dem ihn die Krankenkasse versorgt hatte, war zwar für den Schulsport ausreichend, nicht aber für das Basketballspiel im Verein.

Das BSG hat einen Anspruch auf den Rollstuhl für den Vereinsbasketball nach $\S 33$ Abs. 1 Satz 1 SGB V verneint. Beim mittelbaren Behinderungsausgleich schulde die Kasse nur einen Basisausgleich, nicht aber einen Ausgleich im Sinne des vollständigen Gleichziehens mit den letztlich unbegrenzten Möglichkeiten eines gesunden Menschen (Bestätigung der bisherigen Rechtsprechung zum unmittelbaren und mittelbaren Behinderungsausgleich ${ }^{4}$ ).

2 Az. L 8 SO 129/09.

3 Az. B 3 KR 10/10 R.

4 Vgl. BSG Urteil vom 16.9.2004 - B 3 KR 20/04 R - C-Leg II. 
Durch das Übereinkommen der Vereinten Nationen über die Rechte von Menschen mit Behinderungen ergebe sich keine weitere Leistungsverpflichtung der Krankenkassen. Aus der UN-Konvention resultiere kein subjektiv-öffentliches Recht, ein konkretes und der persönlichen Mobilität dienendes Hilfsmittel von einem bestimmten Leistungsträger verlangen zu können. Die Bundesrepublik trage dem Zweck der UN-Konvention, den vollen und gleichberechtigten Genuss aller Menschenrechte und Grundfreiheiten durch alle Menschen mit Behinderungen zu fördern, zu schützen und zu gewährleisten sowie die Achtung der ihnen innewohnenden Würde zu fördern (Art. $1 \mathrm{UN}$ Konvention) ausreichend durch das gegliederte Leistungssystem des SGB und insbesondere durch dessen Neuntes Buch (Rehabilitation und Teilhabe behinderter Menschen - SGB IX) Rechnung. Weitergehende Einzelansprüche würden - zumindest im SGB V - durch die UN-Konvention nicht begründet.

Die UN-Behindertenkonvention wird in den kommenden Jahren zu einem Paradigmenwechsel weg von der Funktionsstörung hin zur Teilhabestörung führen. In diesem Rahmen wird es wohl dazu kommen, dass die von der Rechtsprechung entwickelte Unterscheidung von unmittelbarem und mittelbarem Behinderungsausgleich auf den Prüfstand gestellt wird.

\section{Inklusionsziel Bildung}

In einem anderen Fall, der dem Bayerischen LSG vorlag, ging es um zwei gehörlose sechsjährige Mädchen. Sie besuchten die Grundschule und nicht, wie vom Sozialhilfeträger gewollt, die Förderschule. Für den Inklusionsunterricht an der Regelschule benötigten sie die Hilfe von Gehörlosen-Sprachdolmetschern. Deren Kosten zu tragen, versagte der Sozialhilfeträger.

Mit seinen Beschlüssen vom 02.11.20115 konstatierte das LSG, Inklusion werde nicht vordergründig durch den Besuch der Regelschule hergestellt. Bildung als Ziel des Schulbesuchs sei mehr, als nur die funktionellen Fertigkeiten zu erwerben, wie Lesen, Rechnen und Schreiben. Das Ziel der integrativen Teilhabe an der Gemeinschaft als individuell entwickelte Persönlichkeit (Inklusion) lasse sich im konkreten Falle eher an der Förderschule erreichen.

Auch wenn der Senat entschieden hat, die UN-Behindertenrechtskonvention führe nicht zu einem unmittelbaren Rechtsanspruch auf Eingliederungshilfe, können beide Entscheidungen als Meilenstein gesehen werden. Das Gericht wendet nämlich die UNBehindertenrechtskonvention an. Und es stellt klar, dass sich ein angemessener Bedarf bei einer Eingliederungsmaßnahme nicht schon wegen Mehrkosten verneinen lässt. Ein Hauptsatz der Entscheidungen besagt nämlich, dass unter angemessener Schulbildung

5 Az. L 8 SO 165/11 B ER und L 8 SO 164/11 B ER. 
iSd $\S \S 53,54$ SGB XII alles zu verstehen ist, was der Erreichung des Ziels, der Integration in die Gesellschaft, dient.

Beide Entscheidungen waren im vorläufigen Rechtsschutz ergangen. Mittlerweile ist ein Hauptsacheverfahren durch gerichtlichen Vergleich beendet worden.

\section{Kein Cialis zulasten der Gesetzlichen Krankenversicherung}

In einem Urteil vom 6.3.20126 hatte das BSG über das Begehren eines Klägers auf Gewährung des von der Versorgung durch die gesetzliche Krankenversicherung ausgeschlossenen Arzneimittels „Cialis“ zu entscheiden. Der Kläger litt an einer erektilen Dysfunktion als Folge einer chronisch progredienten Multiplen Sklerose und sah in der Nichtgewährung des Arzneimittels unter anderem eine Verletzung des Art. 25 UN-BRK (Recht auf Gesundheit).

Im Ergebnis hat das BSG einen Anspruch auf besagtes Arzneimittel zwar verneint. Der gesetzliche Leistungsausschluss nach dem SGB V kollidiere weder mit Art. 25 UNBRK noch verstoße er gegen das Diskriminierungsverbot des Art. 5 Abs. 2 UN-BRK oder des Art. 3 Abs. 3 Satz 2 GG, weil er nicht an eine Behinderung im konventionsoder verfassungsrechtlichen Sinne anknüpfe, sondern alle Fälle der Erkrankung erfasse. Dessen unbeschadet hat sich das BSG aber hier sehr ausführlich Rang und Bedeutung der UN-BRK innerhalb der deutschen Rechtsordnung verankert.

\section{Zusammenfassung und Ausblick}

Der Blick auf diese fünf Entscheidungen zeigt verlässlich, dass die Sozialgerichte den Rechtsanspruch auf Inklusion nicht nur erkennen, sondern umsetzen und im Einzelnen konkretisieren. Ob Hilfen zur Teilhabe

- erforderlich,

- angemessen und

- notwendig sind,

wird künftig mehr und mehr nach den Grundsätzen des Rechtsbegriffes der Inklusion entschieden werden. Hier stellen sich die Sozialgerichte ihren Aufträgen zur Konkretisierung und zur Fortbildung des Rechts der Behinderten. Definitionsauftrag und Definitionshoheit bedeutet aber auch für die Sozialgerichte eine besondere Definitionsverantwortung. Der gilt es, gerecht zu werden.

6 Az. B 1 KR 10/11 R. 


\title{
Behindertenrecht und Behindertenpolitik in der Kommune aus kommunaler Sicht
}

\author{
Helmut Kneppe
}

I. Einleitung 141

1. Sozialraum 141

2. Personenzentrierung 142

3. Aufgabenverständnis der Kommunen 142

II. Kommentierung aus kommunaler Sicht 143

1. Ausgangspunkte der Ausführungen 143

2. Die Realisierung - aus dem Blickwinkel eines kommunalen Praktikers $\quad 144$

$\begin{array}{ll}\text { III. Fazit } & 147\end{array}$

\section{Einleitung}

Der Kommentar erfasst folgende Aspekte: Unter dem Eindruck der bisher vorgetragenen Tagungsbeiträge insgesamt werde ich in aller Kürze auf drei Stichworte eingehen. Diese Stichworte spreche ich aus meinem Blickwinkel ganz praktischer Planungsund Umsetzungsbelange im Sozialraum an. Hierbei bitte ich um ihr Verständnis für die eben wegen der Kürze an der Oberfläche gebliebene Argumentation:

\section{Sozialraum}

Aus meiner von praktischen Zusammenhängen geprägten Sicht ist der Sozialraum für den betroffenen Menschen (Adressaten einer sozialen Leistung) in der Regel keine universell und über bestimmte Koordinaten objektiv zu beschreibende absolute Fläche bzw. kein absoluter Raum. Trotzdem ist ein Sozialraum natürlich räumlich zu verstehen und hat insoweit auch die Aufgabe, räumliche Bezüge und Zusammenhänge herzustellen. Er ist in seiner konkreten Gestaltung immer abhängig von objektiven und subjektiven Rahmenbedingungen des einzelnen (behinderten) Menschen und seinem individuellen (Lebens-) Umfeld. 
Wie auch immer sich Gemeinschaften, Gesellschaften oder auch Staaten organisiert haben, die sozialen Belange der Menschen, ihrer Familien und auch der Gemeinschaften selbst sind nach meiner Kenntnis von der Organisationsebene wahrgenommen worden, die ihnen am nächsten war. Das Grundprinzip dieser örtlichen Kompetenzzuweisung, der Beauftragung der örtlichen Ebene nah am Menschen, haben wir in unsere heutige Situation übernommen:

Der Sozialraum ist der Ursprung und das Urgeschäft der Kommunen.

Im Sozialraum findet der Mensch die Wertschätzung, Kompetenzen, Strukturen, Leistungen, Angebote, Menschen und Partner, um sein Leben nach seinen Möglichkeiten unbehindert und selbstbestimmt führen und am Leben in seinem Sozialraum teilhaben zu können. Der Sozialraum ist sein gelingendes Lebensumfeld „nah am Menschen“.

Die in und für dieses Lebensumfeld zuständigen Akteure gelingender Teilhabe arbeiten in diesem Sinne vernetzt und koordiniert miteinander. Ihnen gelingt es, bedarfsgerecht Infrastruktur-, Geschäftsmodellentwicklung und Hilfeplanung im Einzelfall zu verbinden.

\section{Personenzentrierung}

Personenzentrierung verstehe ich als eine wichtige Perspektive für die Anforderungen an die Gestaltung gelingender Sozialleistungen. Personenzentriert interpretiere ich dementsprechend i.S. der Kundenperspektive der Balanced-Score-Card für soziale Organisationen (herausgearbeitet für not for profit Organisationen ${ }^{1}$ ).

Das heißt, im Rahmen der gesetzlich geregelten Ansprüche und Möglichkeiten sind der (behinderte) Mensch und seine Perspektive (Bedarfe, Lebensentwurf) die inhaltlich gestaltende Bezugsebene. Es sind daher konsequent und dem Grunde nach konsistent auch die Budgets auf die Person und/oder auf den Sozialraum bezogen zu gestalten.

Hieraus folgt: Nicht der Sozialraum oder der/die Sozialplaner/innen stehen im Mittelpunkt, sondern der (behinderte) Mensch und seine Bedarfe im Kontext sozialräumlicher Belange. Ich grenze „,personenzentriert“ in diesem Sinne inhaltlich zu den Begriffen „struktur- und prozesszentriert“, „kompetenzzentriert“ sowie „,ressourcen- und finanzzentriert" ab.

\section{Aufgabenverständnis der Kommunen}

Die erfolgreiche Steuerung des Ressourceneinsatzes bei knappen Ressourcen kann nur gelingen, wenn die Kommunen schon zu einem Zeitpunkt die Zugänge in soziale Leistungen aktiv gestalten, zu dem wir aufgrund der gesetzlichen Regelungen noch

1 Stollmann, BSC für soziale Organisation, Regensburg 2008. 
nicht zuständig sind. Unser Aufgabenverständnis muss/müsste also konsequent proaktiv gestaltend und nicht lediglich verwaltend sein.

Zum Beleg, dass rechtliche Grundlagen für ein solches Aufgabenverständnis an einigen Stellen schon heute vorhanden sind, darf ich zustimmend beispielsweise auf die Darstellungen durch Felix Welti und Gerhard $\operatorname{Ig}^{2}$ Bezug nehmen.

Eine solchermaßen aktiv gestaltende Aufgabenwahrnehmung ist in der Praxis jedoch offensichtlich nicht konsistent zu haushaltsrechtlichen Regelungen und Entscheidungen. Finanzaufsicht stuft mit dem Ziel der Haushaltskonsolidierung die Wahrnehmung derart präventiv angelegter Maßnahmen als „freiwillige Aufgabe“ ein und gestattet diese im Kontext einer Haushaltssicherung oder eines Nothaushaltes in der Regel nicht.

Zahlreiche gute Ansätze zur Wahrnehmung einer modernen Sozialplanung und Steuerungsunterstützung stoßen in einer solchen Lage an die Grenzen des tatsächlich Machbaren.

Aus meiner Sicht müssen hier auch wir Kommunen uns bewegen und verstärkt den tatsächlichen Willen und Mut aufbringen, aktiv zu gestalten. Wir dürfen bei aller berechtigten Anmahnung bedarfsgerechter Ressourcen uns nicht auf eine Opferrolle beschränken und eine entsprechende Mentalität entwickeln.

\section{Kommentierung aus kommunaler Sicht}

\section{Ausgangspunkte der Ausführungen}

a) Soziale Rechte, Ansprüche und Leistungen haben in der Regel die Aufgabe, ökonomische und/oder nicht ökonomische Beratungs-, Assistenz- und Hilfebedarfe der betroffenen Menschen sowie auch ihrer Familien bzw. persönlichem Umfeld zu befriedigen bzw. Beiträge dazu zu leisten.

Sozialpolitisches Wirkungsziel der zu diesem Zweck begründeten Rechte, Ansprüche und Leistungen ist es, die eigenständige und selbstverantwortliche Lebensführung und Teilhabe der Betroffenen zu fördern bzw. diese zu ermöglichen.

b) Zur Erreichung dieser Ziele werden auf Gesetzesebene und unterhalb dieser Anspruchsvoraussetzungen, (angemessene) Beratungs-, Assistenz- und Hilfeleistungen sowie der berechtigte Personenkreis abstrakt beschrieben und in Form von Ansprüchen und Rechten (z.B.) als persönliche -, Sach- oder Geldleistung artikuliert und für die Berechtigten mehr oder weniger formgebunden verfügbar gemacht.

Die Realisierung obliegt sehr vielfältig aufgestellten und positionierten Sozialleistungsträgern. Ihre Leistungserbringung ist in der Regel nicht im wünschenswerten Maße vernetzt.

2 Siehe deren Beiträge, S. 87 und S. 119 in diesem Band. 
Den Kommunen (kreisfreie Städte, Kreise, kreisangehörige Städte und Gemeinden, kommunale Verbände) kommt in diesem Gesamtsystem aus dem Blickwinkel der Leistungsberechtigten strukturell, prozessbezogen und inhaltlich eine wichtige Position zu. Die betroffenen Menschen weisen ihnen eine hohe Lösungs- und Handlungskompetenz zu.

c) Unsere Sozialleistungssysteme stehen unter einem enormen Druck. Die Systeme selbst und deren Planungs-, Aufgaben- und Kostenträger werden zunehmend an ihre Leistungsgrenzen und auch darüber hinaus geführt. Schon jetzt bzw. in absehbarer Zeit werden die verfügbaren Ressourcen, Kompetenzen und Möglichkeiten überfordert sein. Es sei denn, wesentliche Rahmenbedingungen des „Gelingens sozialer Leistungserbringung" werden mit nachhaltiger Wirkung verändert.

Wir müssen daher schon heute das Leistungs-, Organisations- und Finanzierungssystem im $\mathrm{Ob}$ und im Wie gründlich hinterfragen!

d) Aus meinem Blickwinkel des Praktikers führt die oben beschriebene Gefahr der Überforderung der Systeme zunehmend zu Reaktionen auf allen Ebenen.

Auf der kommunalen Ebene reichen diese von der Etablierung aktiv gestaltender präventiv wirkender Konzepte bis hin zu absolut destruktiven Entscheidungen im Rahmen der Haushaltskonsolidierung, die Auswirkungen bis auf die Leistungsebene im Einzelfall haben können. Ganz entscheidende Bedeutung haben hierbei die wirtschaftliche Leistungsfähigkeit der Kommunen und die darauf folgenden Reaktionen.

Destruktive und aus der Sicht der Betroffenen sogar kontraproduktiv wirkende Einzelentscheidungen müssen konstruktiv in Frage gestellt bzw. beendet werden.

Hieraus folgt: Wir brauchen auf der strategischen Entscheidungs-, Planungs- und Steuerungsebene und der operativen Leistungsebene die Entwicklung neuer strategischer und ganzheitlich angelegter Handlungskonzepte. Ich denke, hier liegt ein wichtiges Aufgabenfeld für die Sozialleistungsträger, insbesondere auch die Kommunen, sowie Wissenschaft und Forschung. Der von Wissenschaft und Forschung sowie manchen Sozialleistungsträgern schon seit einigen Jahren eingeschlagene Weg der Entwicklung aktiver Planungs- und Steuerungskonzepte - aus meiner Sicht ganz bewusst auch in Anlehnung an den „betriebswirtschaftlichen Methodenkoffer“ - sollte intensiviert werden.

\section{Die Realisierung - aus dem Blickwinkel eines kommunalen Praktikers}

a) Die Erreichung der gewollten Ziele/Wirkung gelingt, wenn die Leistungen

_ „nah am Menschen“ und

- tatsächlich, verlässlich, qualitätsvoll, wertschätzend und finanziert sind.

„Nah am Menschen“ heißt: bedarfsgerecht; ortsnah; vernetzt und eingebunden; finanziert. „Nah am Menschen“ heißt daher zwingend auch: Soziale Leistungserbringung 
muss personenzentriert und ganzheitlich gestaltet sein. Personenzentriert und ganzheitlich gestaltet bedeutet aus der Sicht der betroffenen Menschen und der Sozialleistungsträger:

- Die Infrastruktur, das Leistungsangebot, die Geschäftsmodelle müssen dem entsprechend entwickelt und für die betroffenen Menschen im Sozialraum verfügbar sein (Stichwort: Care-Management);

- die Leistung, der Leistungsprozess kommen passend im jeweiligen Quartier an und entfalten die gewünschte Wirkung (Stichwort: Case-Management);

- die für eine wertschätzende Leistungserbringung notwendigen Kompetenzen und Ressourcen sind im erforderlichen notwendigen Maße verlässlich und bezahlbar vorhanden.

Das Gelingen sozialer Leistungen entsprechend diesen Maßgaben zu organisieren, bedarf einer diesen Anforderungen entsprechenden Sozialplanung, Sozialraumplanung sowie Organisation und Steuerung der Leistungserbringung, also einer planvollen, zielgerichteten, partnerschaftlichen und disziplinübergreifenden Interaktion aller an der sozialen Leistungserbringung beteiligten Akteure.

b) Die Ebene, auf der alle beteiligten Akteure, die notwendigen Kompetenzen und die betroffenen Menschen zusammenkommen bzw. vertreten sind, ist die Kommune.

Nur auf kommunaler Ebene, bei und mit den Menschen vor Ort, können soziale Leistungen ihre gewollte Wirkung in vollem Umfang entfalten. Letztlich werden dort Ansprüche in konkrete Leistungen übersetzt. Nur auf der kommunalen Ebene haben soziale Leistungen einen persönlichen Ansprechpartner und sind nicht anonym. (Stichwort „Dienstleistung aus einer Hand“). Hierbei ist es ein Qualitätsmerkmal und nicht Hindernis, dass die kommunale Ebene Unterebenen aufweist und in dieser Republik sehr unterschiedlich gestaltet ist. Unsere vielfältigen kommunalen Strukturen sind letztlich auch Garanten für die Nähe zum Menschen. Die Kommunen sind die „geborenen“ (und legitimierten) Gestalter ihrer Sozialräume und Partner ihrer Menschen, Familien und regionalen Akteure. Der Sozialraum ist das Urgeschäft der Kommunen.

c) Aus der bisherigen - ganz bewusst nicht juristisch, sondern sozialpolitisch aus der Sicht der betroffenen Menschen vorgenommenen - Ableitung folgt zwangsläufig, dass sich damit auch eine entsprechende Verpflichtung und Aufgabenstellung der kommunalen Akteure ergibt. Unter Aufgreifen des von Gerhard $\mathrm{Igl}^{3}$ dargestellten Gedankens darf auch aus meiner Sicht eine „Entpflichtung“ bzw. „Selbstentpflichtung“ der Kommunen nicht Raum greifen.

Ich sehe im Rahmen der aktuellen Inklusionsdiskussion die Entwicklung, dass wir Kommunen uns formal hinter den noch ausstehenden Regelungen des Bundes und der Länder sowie unseren Zuständigkeiten verstecken. Findet auch hier wieder eine Selbstentpflichtung statt?

3 S. 119 in diesem Band. 
Jedoch darf es auch für Bund und Land keine Selbstentpflichtung zu Lasten der Kommunen geben. Bund und Land überfordern ihre kommunalen Partner, indem sie kommunale Leistungen beauftragen und die erforderlichen Mittel nicht, nicht ausreichend bzw. nur ,ausreichend“ im Sinne einer recht eigenen Sichtweise/Bedarfsermittlung zur Verfügung stellen.

d) Was ist vor Ort notwendig, damit soziale Leistungserbringung gelingt? Die nachfolgenden Beispiele beziehen sich auf Klarheit und Wertschätzung in Aufgabe, Auftrag, Zielen und Wirkungen, Zielgruppe, Verantwortlichkeiten, Terminen und Ressourcen:

- Benennung der Aufgabe;

- aus welchem Grund und Anlass greifen wir diese auf;

- welche Wirkungen und Ziele verfolgen wir?;

- für wen, Zielgruppe?;

- welche Ist-Situation wollen wir in welche Soll-Situation entwickeln?;

- mit welchen Maßnahmen, Projekten, Produkten erreichen wir die Ziele, die Wirkungen?;

- wer ist verantwortlich und kümmert sich um Planung, Produktion, Evaluation und Steuerung?;

- welche Partner und Beteiligten sind einzubinden?;

- womit wird die Aufgabe, der Auftrag erledigt, was ist erforderlich? Das bezieht sich auf Personal (Kompetenzen), Sach-, Betriebs- und Hilfsmittel, IT TUIV, sowie die Finanzen.

Diese Punkte sind abgeleitet aus verschiedenen Steuerungsansätzen und bedürfen inhaltlich und methodisch der Konkretisierung. Einer Anforderung die sich zuvorderst an den Aufgabenverantwortlichen wendet und diese zur Schaffung leistungsfähiger Angebote verpflichtet.

Insbesondere die Bereitstellung der für die Aufgabenwahrnehmung notwendigen Ressourcen ist in der Praxis häufig mangelhaft ausgeprägt. Für mich steckt darin auch ein Signal mangelnder Wertschätzung und mangelnden Respekts gegenüber Betroffenen und Handelnden.

e) Eine ehrliche Analyse der allgemeinen und örtlichen Ausgangssituation muss die folgenden Rahmenbedingungen vor Ort erfassen:

- Demografische Entwicklung, heruntergebrochen auf die örtliche Situation;

- Knappheit der verfügbaren Ressourcen, soziale Leistungen im Wettbewerb um kommunale Ressourcen;

- Aufgaben- und Methodenkritik, Kosten-Nutzen-Analysen;

- neues kommunales Finanzmanagement NkF, Kaufmännische Buchführung, Doppik, Verwaltungsunternehmen, unternehmerische Führung;

- Aufgabenbezogene Bestandsaufnahme, Ist-Erhebung, Repräsentativbefragungen. 
Der politische und fachliche Gestaltungswille der Kommune muss von den kommunalen Gremien beschlossen, kommuniziert und verlässlich gelebt werden - als politische Kommune und als Sozialleistungsträger.

f) In der örtlichen Situation ist Ganzheitlichkeit und gemeinsame Zielorientierung in ländlichen Gebieten methodisch anders herzustellen als in städtischen Gebieten. Dies liegt nicht nur an den Anforderungen der Versorgung in der Fläche. Das liegt auch am „Kirchturmdenken“.

In den Städten, Gemeinden und Kreisen, Regiestellen und Servicestellen Soziales sollen örtliche „Kümmerer“ etabliert werden. Sie sollen aus den Rathäusern und Kreishäusern heraus mit den betroffenen Menschen, den Aufgaben- und Kostenträgern, den Dienstleistern und dem bürgerschaftlichen Engagement gemeinsam passgenaue Infrastruktur, Strategien und Angebote entwickeln.

\section{Fazit}

Gelingende Sozialpolitik und gelingende Sozialleistung zu gewährleisten und dies auch noch zur Zufriedenheit der Leistungsempfänger, ihres Umfeldes und der Aufgaben- und Kostenträger ist eine „Übung für Fortgeschrittene“.

Grundvoraussetzungen des Gelingens sind

- die Verankerung der Leistungserbringung im Sozialraum

- und dem entsprechend die Akzeptanz und positive gestaltende Annahme dieser Aufgabe durch die Kommunen.

Die schon bestehenden gesetzlichen und auch untergesetzlichen Regelungen, sich dieser Aufgabe im erforderlichen Maße legitimiert anzunehmen, sind aus meiner kommunalen Sicht vielfältig. Sie werden derzeit durch uns Kommunen sehr unterschiedlich aufgegriffen. In einer Gesamtsicht nutzen wir diese Handlungsfelder und die in ihnen liegenden Gestaltungsräume wohl noch nicht umfassend.

Ich sehe hier wichtige Handlungsfelder und Entwicklungsmöglichkeiten für uns Kommunen sowie Gestaltungsraum für den Gesetz- und Verordnungsgeber, Planung, Koordination und Vernetzung personenzentrierter Hilfeleistung im örtlichen Sozialraum kommunal verlässlich zu verankern.

Im Sinne einer Zielbeschreibung bedeutet dies soziale Leistungserbringung in modernen Dienstleistungsunternehmen zu organisieren, zu steuern und sicherzustellen.

Die Kommunen sind jedoch aus meiner Perspektive für die Wahrnehmung der ihnen obliegenden Aufgaben in der geforderten Qualität insgesamt unterfinanziert.

Es dürfte nicht zulässig sein, ihnen Aufgaben zu übertragen, bestehende Aufgaben zu erweitern, ohne die Aufgabenfinanzierung verlässlich zu regeln. Alternativ wäre die geforderte Produktqualität anzupassen. 
Wir Kommunen haben hierbei auch weiterhin unseren Beitrag der eigenen Strukturund Prozessoptimierung zu erbringen.

Die Wahrnehmung, Übertragung bzw. Erweiterung von Aufgaben (z.B. als Sozialleistungsträger) muss getragen sein von einer eindeutigen Auftragslage, Klarheit in der Zielsetzung, in den Zuständigkeiten und in der Ausstattung mit Ressourcen.

Eine der Gesetzesentscheidung vorgelagerte gründliche und umfassende Produkt- bzw. Leistungsplanung, Ziel- und Maßnahmenabstimmung sowie auch Planung der notwendigen Transfermittel und einzusetzenden Ressourcen sollte eine wichtigere Position einnehmen.

Sozialleistung gelingt, wenn sie personenzentriert gestaltet, im Sozialraum verankert ist, nah am Menschen ankommt sowie verlässlich, qualitativ hochwertig, wertschätzend und finanziert erbracht wird. Aus der praktischen Sicht ergibt sich zum heutigen Thema folgende Ableitung:

Für mich und für die Kommunikation in meinen kommunalen Bezügen nehme ich aus dieser Fachtagung ein hohes Maß, ja sogar die Aufforderung mit, den aktiv gestaltenden Ansatz unserer kommunalen Sozialpolitik weiter zu verfolgen, sogar noch zu verstärken.

Alter und Behinderung stehen in den Zusammenhängen dieser Fachtagung für mich beispielhaft. Sie können - wenn auch nicht beliebig - so doch durch andere Beschreibungen von Lebenslagen ersetzt werden. Daraus folgt, dass meine Ausführungen eine auch über das Thema hinausgehende Dimension haben.

Wenn sich das Sozialleistungsrecht in drei Dimensionen (Schutz bei Pflegebedürftigkeit, medizinische Rehabilitationsfähigkeit, spezielle Gesundheitsleistungen) abbildet, so resultiert daraus, dass das Sozialleistungsrecht das Entstehen des zugeordneten Leistungsanspruches an eine defizitäre Lebenssituation bindet.

Der auslösende Zustand muss die Teilhabe, die Lebensqualität des Betroffenen beeinträchtigen. Sozialleistung repariert demnach und gestaltet nur eingeschränkt.

Sozialpolitisch muss aus alledem folgend sehr viel stärker als dies heute schon möglich ist und geschieht Präventionsleistung vor Eintritt des Leistungsanspruches verankert werden. Besonders gilt dies aus meiner Sicht für Sozialleistungen, die nicht beitragsfinanziert sind wie z.B. die Jugendhilfe, die Sozialhilfe, die Behindertenhilfe, die Wohnungshilfe.

Ziel sollte sein, den Eintritt des Leistungsfalles zu verhindern bzw. mindestens zu verzögern, d.h., den Zugang, den Verbleib und das Verlassen in/von Sozialleistungen aktiv zu steuern.

Nur auf der kommunalen Ebene im örtlichen Sozialraum laufen all diese Stränge zusammen. Es bedarf einer ganzheitlich angelegten Planung, Vernetzung und Koordination der örtlichen Akteure. Ansprüche, Bedarfe, Strukturen und Prozesse müssen zusammengeführt werden.

In der aktuellen Ausgangslage ist es wesentlich an den Kommunen, diesen Handlungs- 
bedarf aufzugreifen und weiter zu entwickeln, und zwar über Zuständigkeits- und Aufgabengrenzen hinweg.

Auch aus der kommunalen Sicht teile ich den in dieser Fachtagung an zahlreichen Stellen herausgearbeiteten und dargestellten Handlungsbedarf, gelingende Teilhabe im Sozialraum aktiv aufzugreifen und zu gestalten. Dies bedarf einer verstärkten und gemeinsamen Anstrengung aller Beteiligten, aller Akteure im Sozialraum. 
2. Kinder und Jugendliche mit Behinderungen 


\title{
Die ,große Lösung“ - eine unendliche Geschichte?
}

\author{
Reinhard Wiesner
}

$\begin{array}{ll}\text { I. Einleitung } & 154\end{array}$

II. Das SGB VIII und die kleine Lösung 154

1. Die Entwicklung eines selbstständigen Behindertenrechts 154

2. Die kleine Lösung als Ergebnis der Diskussion zur Reform des Kinder-
und Jugendhilferechts

III. Die Diskussion um die große Lösung 156

1. Die große Lösung - zurück auf der politischen Agenda 156

2. Der Transfer von Finanzen und Personal 157

3. Die unterschiedlichen Leistungsvoraussetzungen 158

4. Der altersbedingte Übergang in die Sozialhilfe 159

5. Die unterschiedliche Kostenbeteiligung der Eltern 159

IV. Der inklusive Ansatz und seine Herausforderungen 160

1. Das Verständnis der UN-Behindertenrechtskonvention 160

2. Von der Integration zur Inklusion 161

3. Das Konzept der Inklusion als Auftrag für alle Leistungssysteme 161

V. Inklusion: Kinder- und Jugendhilfe neu denken? 162

1. Die inklusive Ausgestaltung des Leistungssystems 162

2. Leistungen für Eltern mit Behinderung 163

3. Anforderungen an die Angebotsstruktur 163

4. Flexible Übergänge in die Sozialhilfe 164

5. Leistungsfähigkeit der Jugendämter 164

6. Das Ende der Ausfallbürgschaft für andere Systeme 164

VI. Zum Stand der (politischen) Debatte 165

1. Die Positionierung des 13. Kinder- und Jugendberichts 165

2. Der Nationale Aktionsplan zur Umsetzung der
UN-Behindertenrechtskonvention

VII. Inklusion als gesellschaftspolitische Herausforderung 166

$\begin{array}{ll}\text { VIII. Literatur } & 167\end{array}$ 


\section{Einleitung}

Mit der Ratifizierung der UN-Konvention über die Rechte von Menschen mit Behinderungen im Jahr 2009 hat in Deutschland auch die Debatte über eine Neujustierung des Systems der Kinder- und Jugendhilfe an der Schnittstelle zur Sozialhilfe (große Lösung) neuen Auftrieb erhalten, nachdem der Gesetzgeber im Rahmen der Neuordnung des Kinder- und Jugendhilferechts im Jahre 1990 nur die kleine Lösung realisiert hatte. Ist bereits die Umsetzung der großen Lösung mit einem erheblichen Transfer an Finanzen und fachlichen Ressourcen von der Sozial- zur Kinder- und Jugendhilfe verbunden, so sind vor dem Hintergrund des Inklusionsparadigmas, das der UN-Behindertenrechtskonvention zu Grunde liegt, alle staatlichen Leistungssysteme in diesem Hinblick auf den Prüfstand zu stellen. Die große Lösung kann nur ein erster Schritt hin zu einer neuen Architektur des Leistungssystems Kinder- und Jugendhilfe sein.

\section{Das SGB VIII und die kleine Lösung}

\section{Die Entwicklung eines selbstständigen Behindertenrechts}

Das Behindertenrecht wurde in Deutschland stufenweise entwickelt. Den Anfang bildeten landesgesetzliche Regelungen zur sog. Krüppelfürsorge - also Regelungen für Personen mit einer körperlichen Beeinträchtigung. Von diesen Gesetzen ist vor allem das preußische Krüppelfürsorgegesetz aus dem Jahre 1920 zu erwähnen. Es sah Beratungsstellen in jedem Stadt- und Landkreis vor und verpflichtete Ärzte und Hebammen, Verkrüppelungen bei unter 18-Jährigen dem Jugendamt anzuzeigen ${ }^{1}$. Nachdem im Bundestag bereits im Jahre 1951 eine Harmonisierung des zersplitterten Landesrechts gefordert worden war, wurde erst im Jahre 1957 ein erstes Körperbehindertengesetz verabschiedet, das im Jahre 1961 Eingang in das Bundessozialhilfegesetz im Abschnitt „Hilfe in besonderen Lebenslagen“ fand. Die Kooperationsverpflichtung mit dem Jugendamt wurde nicht aus dem Landesrecht übernommen ${ }^{2}$. Die bis dahin auf körperliche und geistige Behinderungen begrenzte Eingliederungshilfe wurde schließlich im Jahre 1976 auf die seelische Behinderung erweitert.

1 Hasenclever, Jugendhilfe und Jugendgesetzgebung seit 1900, Göttingen 1978, S. 97.

2 Hasenclever (Fußn. 1), S. 189. 


\section{Die kleine Lösung als Ergebnis der Diskussion zur Reform des Kinder- und Jugendhilferechts}

Mit der Neuordnung des Kinder- und Jugendhilferechts durch das KJHG im Jahre 1990 kam eine zwanzigjährige Debatte zu einem vorläufigen Ende. Im Rahmen dieser Neuordnung, deren Kernstück (Art. 1 KJHG) das Achte Buch Sozialgesetzbuch Kinder- und Jugendhilfe (SGB VIII) war, wurde die „kleine Lösung“ umgesetzt, d. h. die Zuständigkeit für Kinder und Jugendliche mit seelischer Behinderung aus der Gesamtzuständigkeit der Eingliederungshilfe im (damaligen) Bundessozialhilfegesetz (BSHG) herausgelöst und der Kinder- und Jugendhilfe übertragen. Konkret sichtbar wurde diese Gesetzesänderung in der Einführung eines neuen Leistungstatbestandes, des $\S 35$ a SGB VIII, nachdem zunächst die Eingliederungshilfe als Unterfall der Hilfe zur Erziehung in $\S 27$ SGB VIII geregelt war. Mit diesem Schritt folgte der Gesetzgeber Reformvorschlägen, wie sie etwa der Deutsche Verein für öffentliche Fürsorge in seinen Thesen zu einem neuen Jugendhilferecht ${ }^{3}$ vorgestellt hatte. Schon damals hatte es Stimmen in der Fachszene gegeben, die sich für eine umfassende Zuständigkeit der Kinder und Jugendhilfe für alle jungen Menschen ausgesprochen hatten.

Grund für diese „kleine Lösung“ war die Erkenntnis, dass die Schnittmengen zwischen seelischer Behinderung einerseits und Verhaltensauffälligkeit und Entwicklungsstörung andererseits so groß sind, dass eine praktikable Zuordnung der Symptomatik und des daraus resultierenden Hilfebedarfs zur Eingliederungshilfe (wegen der seelischen Behinderung) bzw. zur Kinder- und Jugendhilfe (wegen der Störung der Entwicklung) nicht möglich ist ${ }^{4}$. Die ,große Lösung“ wurde wegen der damit verbundenen Vollzugsprobleme, auf die noch näher einzugehen sein wird, aber auch wegen der Ablehnung durch die Behindertenverbände zurückgestellt. Die Zuweisung junger Menschen mit Behinderung zum Jugendamt wurde - und wird zum Teil bis heute - dort als Stigma empfunden, da sie elterliches Versagen in der Erziehung der Kinder impliziere. Dabei wird nicht berücksichtigt, dass sich mit der Neuordnung des Kinder- und Jugendhilferechts das Aufgabenverständnis der Kinder- und Jugendhilfe im Sinne eines Perspektivenwechsels hin zu einer stärker präventiven, familienunterstützenden Funktion geändert hat.

Manche Eltern sind allerdings auch (bis heute) nicht bereit, sich auf die systemische Sichtweise der Kinder- und Jugendhilfe einzulassen, die die Beeinträchtigung des Kindes im Zusammenhang mit dem Erziehungsverhalten der Eltern sieht und damit (auch) von ihnen Verhaltensänderungen erwartet.

3 Deutscher Verein für öffentliche und private Fürsorge, Thesen zu einem neuen Jugendhilferecht, Frankfurt am Main 1973.

4 Wiesner, Eingliederungshilfe für seelisch behinderte junge Menschen als Aufgabe der Jugendhilfe, Zentralblatt für Jugendrecht 1996, S. 199. 
Die „kleine Lösung“ erwies sich von Anfang an als Kompromiss mit vielen Nachteilen, weil sie nach den Formen der Behinderung differenziert, keine überzeugende Antwort auf die Behandlung einer sogenannten Mehrfachbehinderung gibt und im Hinblick auf Kinder und Jugendliche mit körperlicher oder geistiger Behinderung den Zusammenhang zwischen Erziehungskompetenz der Eltern einerseits und Beeinträchtigung des Kindes oder Jugendlichen andererseits vernachlässigt ${ }^{5}$.

So blieb es bis zum heutigen Tag bei einer Zweiteilung - jungen Menschen mit einer körperlichen oder geistigen Behinderung, die der Eingliederungshilfe nach dem SGB XII zugeordnet sind, und jungen Menschen mit einer seelischen Behinderung, die der Kinder- und Jugendhilfe zugeordnet sind. Auch die Schaffung des SGB IX als gemeinsames Dach über den verschiedenen Rehabilitationsträgern konnte diese Kluft nicht beseitigen 6 .

\section{Die Diskussion um die große Lösung}

\section{Die große Lösung - zurück auf der politischen Agenda}

Die Debatte über eine umfassende Zuständigkeit der Kinder- und Jugendhilfe für alle Kinder und Jugendlichen wird schon seit langer Zeit geführt. So befasste sich bereits der Diskussionsentwurf zu einem Jugendhilfegesetz aus dem Jahre 1973 mit dem The$\mathrm{ma}^{7}$. Namhafte Vertreter der Kinder- und Jugendpsychiatrie, aber auch der Kinder- und Jugendhilfe haben sich im Rahmen der Reformdiskussion für eine neue Architektur des Kinder- und Jugendhilferechts ausgesprochen. Behinderte Kinder und Jugendliche sollten nicht länger dem Sondersystem „Behindertenrecht“ zugeordnet werden, sondern der für die Lebenslage von Kindern und Jugendlichen zuständigen Jugendhilfe (sog. große Lösung). Mehrere Sachverständigenkommissionen haben in den von ihnen vorgelegten (Kinder- und) Jugendberichten für die große Lösung votiert ${ }^{8}$.

Diese Forderung gründet nicht nur auf einer anderen Sichtweise auf junge Menschen mit Behinderung, sie ist auch das Fazit aus leidvollen Erfahrungen mit der Klärung des zuständigen Leistungsträgers und daraus resultierenden Streitigkeiten zwischen Trägern

5 Fegert, Kinder und Jugendliche mit Behinderung, Gemeinsames Sonderheft Jugendamt/Zeitschrift für Kindschaftsrecht und Jugendhilfe 2010, S. 13 f. Meysen, Kinder- und Jugendhilfe an allen Schnittstellen: zentrale Anlaufstelle, Ausfallbürge, Netzeknüpfer, Gemeinsames Sonderheft Jugendamt/Zeitschrift für Kindschaftsrecht und Jugendhilfe 2010, S. 21 ff.

6 Wiesner, Die Bedeutung des Neunten Buches Sozialgesetzbuch - Rehabilitation und Teilhabe behinderter Menschen - für die Kinder- und Jugendhilfe, Zentralblatt für Jugendrecht 2001, S. 199 ff.

7 Schwengers, Eingliederungshilfen für seelisch behinderte Kinder und Jugendliche, Stuttgart u.a. 2007, S. $43 \mathrm{f}$.

8 10. Kinder- und Jugendbericht, BT-Dr. 13/11368 S. 280; 11. Kinder- und Jugendbericht, BTDr. 14/8181 S. 229. 
der Sozialhilfe und Trägern der Kinder- und Jugendhilfe, die häufig vor den Verwaltungsgerichten ausgetragen wurden ${ }^{9}$.

Nachdem der Gesetzgeber im Rahmen der Neuordnung des Kinder- und Jugendhilferechts im Jahre 1990 nur die kleine Lösung realisiert hatte, hat das Thema ,große Lösung" in verschiedenen Strukturdebatten wieder neue fachpolitische Aufmerksamkeit erhalten. So wird von Ländern, kommunalen Spitzenverbänden und Behindertenorganisationen seit Jahren eine Neuordnung des Behindertenrechts gefordert. Unter dem Stichwort „Weiterentwicklung der Sozialhilfe“ werden neben einer Stärkung von Selbstbestimmung, Eigenverantwortung und Selbsthilfepotenzialen sowie einer verbesserten Steuerung und Wirkungskontrolle die Herauslösung der Eingliederungshilfe aus der steuerfinanzierten öffentlichen Fürsorge und ihre Verankerung in einem vom Bund (mit-)finanzierten Leistungssystem gefordert. Um die Zustimmung der Länder zum so genannten Fiskalpakt zu erreichen, will der Bund die Länder bei ihren Sozialausgaben entlasten. So haben sich Bund und Länder über die Verabschiedung eines Bundesleistungsgesetzes für die Eingliederungshilfe in der kommenden Legislaturperiode verständigt. Hier sollen die Länder jährlich um vier Mrd. Euro entlastet werden.

Im Rahmen dieser Grundsatzdiskussion wurde zur Prüfung von Möglichkeiten für eine Neuordnung der Zuständigkeiten zur Sicherung der Teilhabe von Kindern und Jugendlichen mit Behinderungen eine Interkonferenzielle Arbeitsgruppe (Arbeits- und Sozialministerkonferenz - ASMK; Jugend- und Familienministerkonferenz - JFMK; Kultusministerkonferenz - KMK; Gleichstellungs- und Frauenministerkonferenz GFMK unter Beteiligung des Bundesministeriums für Arbeit und Soziales - BMAS sowie des Bundesministeriums für Familie, Senioren, Frauen und Jugend - BMFSFJ) eingerichtet, die sich eindeutig für die „große Lösung“ ausgesprochen hat ${ }^{10}$. Auch im Koalitionsvertrag für die aktuelle 17. Legislaturperiode wird das Thema - wenn auch nur sehr abstrakt - angesprochen ${ }^{11}$.

\section{Der Transfer von Finanzen und Personal}

Schon in der Debatte der 70er-Jahre über die Realisierung der großen Lösung erwiesen sich die damit verbundenen Fragen des Transfers von Finanzen und Personal von dem einen Leistungssystem in das andere als zentrale Herausforderung, die nach der Kompetenzordnung des Grundgesetzes nicht durch den Bund, sondern durch Länder und Kommunen zu meistern ist. Viel stärker als bei der Erbringung von Geldleistungen hängt die Erbringung personenbezogener sozialer Dienstleistungen von einer an den Zielen des Gesetzes orientierten Leistungssteuerung, insbesondere von einschlägigen

9 Wiesner (Fußn. 6), S. 281.

10 Struck/Porr/Koch, Zeit lassen ... aber ganz schnell anfangen! Zur aktuellen Debatte um die große Lösung, Forum Erziehungshilfen 2010, S. 196 ff.

11 Koalitionsvertrag, S. 71, http://www.cdu.de/doc/pdfc/091026-koalitionsvertrag-cducsu-fdp.pdf. 
fachlichen Kompetenzen, ab. Zielsetzungen des Gesetzes können so durch eine dysfunktionale Verwaltung konterkariert werden. Da die Eingliederungshilfe nach dem SGB XII in den Bundesländern unterschiedlich organisiert (und finanziert) ist, können die Herausforderungen, die mit einer Neuordnung der Zuständigkeiten verbunden sind, nur landesspezifisch identifiziert und bewertet werden. Am einfachsten dürfte sich eine Neuordnung in den Stadtstaaten sowie in den Ländern gestalten lassen, in denen die Eingliederungshilfe kommunalisiert ist und die örtlichen Träger beider Systeme (weitgehend) identisch sind.

Die Aufgabenwahrnehmung setzt aber nicht nur eine entsprechende Finanzausstattung, sondern vor allem auch die notwendige fachliche Kompetenz voraus. Die Feststellung von (spezifischen) Bedarfen von Kindern und Jugendlichen mit körperlicher oder geistiger Behinderung gehörte bisher nicht zu den zentralen Aufgaben der Kinder- und Jugendhilfe. Jeder Neuzuschnitt der Aufgabenverantwortung ist deshalb zwangsläufig mit einem Erwerb zusätzlicher Kompetenzen verbunden. Dies kann durch die Umschichtung von Fachpersonal, das durch die Aufgabenverlagerung in den bisherigen Behörden frei wird, geschehen, aber auch dadurch, dass vorhandenes Personal entsprechend qualifiziert wird. Jedenfalls bedarf es entsprechender Strategien, die gewährleisten, dass Jugendämter die erweiterten Aufgaben auch mit der nötigen Sachkompetenz erfüllen.

\section{Die unterschiedlichen Leistungsvoraussetzungen}

Während § 35a SGB VIII im Hinblick auf den Rechtsanspruch auf Eingliederungshilfe für Kinder und Jugendliche mit einer seelischen Behinderung unmittelbar an den Behinderungsbegriff des SGB IX anknüpft, beschränkt § 53 SGB XII bei der Eingliederungshilfe für Menschen mit körperlicher oder geistiger Behinderung den Rechtsanspruch auf Menschen mit einer wesentlichen Behinderung und stellt die Leistungsgewährung im Übrigen in das pflichtgemäße Ermessen der Behörde. Im Hinblick auf die Folgen einer körperlichen, geistigen oder seelischen Beeinträchtigung für die weitere Entwicklung des Kindes oder Jugendlichen sollte der Gesetzgeber den präventiven Aspekt im Blick haben und sich am Modell des Rechtsanspruchs auf Eingliederungshilfe für Kinder und Jugendliche mit einer seelischen Behinderung orientieren, die allerdings wegen der damit verbunden Senkung der Leistungsvoraussetzungen für Kinder und Jugendliche mit einer körperlichen oder geistigen Behinderung mit Mehrkosten verbunden ist. 


\section{Der altersbedingte Übergang in die Sozialhilfe}

Bei der Realisierung der großen Lösung ist auch zu bedenken, dass (vor allem) körperliche und geistige Beeinträchtigungen (leider) über das Erreichen der Volljährigkeit und der sozialen Integration und damit über die magische Altersgrenze des Kinder- und Jugendhilferechts, das 27. Lebensjahr, hinaus fortdauern. Dieser Befund scheint auf den ersten Blick für eine generelle Zuordnung der Leistungen für junge Menschen mit Behinderung zu einem einheitlichen Behindertenrecht zu sprechen. Bei diesem Konzept, das innerhalb der kommunalen Spitzenverbände immer noch Anhänger findet ${ }^{12}$, wird aber die spezifische Bedeutung der Lebenslage Kindheit und Jugend für die Bewertung der verschiedenen Formen der Beeinträchtigung ausgeblendet. Damit es an dieser altersbezogenen Schnittstelle nicht zu erneuten Zuständigkeitsstreitigkeiten kommt, bedarf es einer praktikablen Abgrenzungsregelung, die sich aber in Form einer Altersgrenze - etwa bei 18 oder 21 Jahren - relativ leicht finden lässt.

\section{Die unterschiedliche Kostenbeteiligung der Eltern}

Schließlich bedarf es auch einer Harmonisierung der Kostenbeteiligung. Im Augenblick ist die Heranziehung von Eltern und jungen Menschen zu den Kosten der Leistung in der Jugendhilfe nach dem SGB VIII und in der Eingliederungshilfe nach dem SGB XII unterschiedlich ausgestaltet. In der Jugendhilfe wirkt (jedenfalls unterschwellig) noch immer das Paradigma fort, dass deren Hilfen von Eltern in Anspruch genommen werden, die ihrer Erziehungspflicht nicht richtig nachkommen können oder wollen. Andererseits war gerade die steigende Inanspruchnahme von Eingliederungshilfe für Kinder und Jugendliche mit seelischer Behinderung nach § 35a SGB VIII Anlass für eine Verschärfung der Heranziehung von Eltern mit höherem Einkommen zu den Kosten bei den individuellen Hilfen nach dem SGB VIII. In der Eingliederungshilfe (nach dem SGB XII) ist die Vorstellung handlungsleitend, dass eine Behinderung ohnehin schon eine schwere Belastung für das Kind und für dessen Eltern bedeute und daher der Staat diese Personen nicht noch zusätzlich finanziell belasten dürfe. Die Heranziehung wird hier auf die häusliche Ersparnis begrenzt und diese pauschaliert (§ 92 SGB XII). Schon bisher sind diese unterschiedlichen Formen der Heranziehung (verfassungs-) rechtlich nicht unproblematisch, wenn man sich vergegenwärtigt, dass Eltern mit gleichem Einkommen bei der Inanspruchnahme von Eingliederungshilfe für Kinder mit seelischer Behinderung stärker zu den Kosten herangezogen werden als bei der Eingliederungshilfe für Kinder mit körperlicher oder geistiger Behinderung.

12 Vorholz, „Große Lösung?“ Zur Diskussion in den kommunalen Spitzenverbänden, Forum Erziehungshilfen 2010, S. $216 \mathrm{ff}$. 
Eine große Lösung ist deshalb nur bei gleichzeitiger Harmonisierung der Kostenbeteiligung denkbar. Die generelle Übernahme der Maßstäbe des Kinder- und Jugendhilferechts würde Eltern von Kindern mit körperlicher oder geistiger Behinderung mit mittlerem oder höherem Einkommen deutlich stärker belasten. Daher ist eher mit einer vermittelnden Lösung oder der Übernahme der Maßstäbe aus dem SGB XII zu rechnen, was dann aber zu Einnahmeausfällen in den öffentlichen Kassen bzw. zu Mehrkosten für die kommunalen Gebietskörperschaften im Bereich der Kinder- und Jugendhilfe führen muss.

\section{Der inklusive Ansatz und seine Herausforderungen}

\section{Das Verständnis der UN-Behindertenrechtskonvention}

Einen neuen Schub hat die Debatte über eine Neujustierung der Zuständigkeiten von Jugend- und Sozialhilfe im Zusammenhang mit dem Inkrafttreten der UNBehindertenrechtskonvention erhalten. ${ }^{13}$ Der Konvention liegt ein Verständnis von Behinderung zugrunde, das diese - bzw. die ihr zugrunde liegende Beeinträchtigung nicht von vornherein defizitär bzw. negativ betrachtet, sondern als normalen Bestandteil menschlichen Lebens und menschlicher Gesellschaft ausdrücklich bejaht und darüber hinaus als Quelle möglicher kultureller Bereicherung wertschätzt (sogenannter Diversity-Ansatz). Die Konvention würdigt das Leben mit Behinderungen als Ausdruck gesellschaftlicher Vielfalt und fordert damit Anerkennung nicht nur für den behinderten Menschen und seine Würde, sondern auch für die durch die Behinderung bedingten besonderen Lebensformen ${ }^{14}$. Behinderung resultiert - so die Sichtweise der Konvention - aus der Beziehung zwischen Personen mit Beeinträchtigungen und den in Grundhaltungen und Umweltfaktoren bestehenden Barrieren mit der Folge, dass dadurch die vollständige und wirksame Beteiligung der Betroffenen auf der Grundlage der Gleichheit mit anderen behindert wird. An die Stelle des medizinischen Modells der Behinderung tritt das menschenrechtliche Model1 ${ }^{15}$. Die Behinderung eines Menschen ist damit keine Eigenschaft, sondern ein Konstrukt - das Ergebnis einer Kontextabwägung. Die Personen haben zwar eine individuelle Benachteiligung, diese wächst sich aber erst dann zu einer Behinderung aus, wenn die Umwelt nicht entsprechend organisiert und

13 Banafsche, Kinder und Jugendliche mit Behinderung zwischen SGB VIII und SGB XII - im Lichte der UN-Behindertenrechtskonvention, Zeitschrift für Kindschaftsrecht und Jugendhilfe 2011, S. $116 \mathrm{ff}$.

14 Bielefeldt, Zum Innovationspotenzial der Behindertenrechtskonvention, 3. Aufl. Berlin 2009, www.institut-fuer-menschenrechte.de. Zinke, Die UN-Behindertenrechtskonvention und ihre Bedeutung für eine Inklusionsperspektive junger Menschen, Forum Erziehungshilfen 2010, S. 212 ff.

15 Degener, Die UN-Behindertenrechtskonvention als Inklusionsmotor, Recht der Jugend und des Bildungswesens 2009, S. $200 \mathrm{ff}$. 
strukturiert ist. Umwelt umfasst einerseits die Haltung der Personen, die im Kontakt zu den Personen mit Beeinträchtigungen stehen, andererseits die Umweltfaktoren, zum Beispiel Barrieren usw. Denkt man die Konvention zu Ende, wird es zwar wohl immer beeinträchtigte Personen geben, aber der Anteil von Menschen „mit Behinderung“ müsste mit der Umsetzung der Konvention tendenziell deutlich sinken.

Die UN-Behindertenrechtskonvention - bestehend aus dem Übereinkommen über die Rechte von Menschen mit Behinderung und einem Fakultativprotokoll - ist am 13. Dezember 2006 verabschiedet worden. Deutschland hat sie am 30. März 2007 unterzeichnet. Seit dem 26. März 2009 ist sie geltendes Recht in Deutschland. Sie reiht sich ein in die Menschenrechtskonventionen. So basieren verschiedene Artikel auf der UN-Kinderrechtskonvention. Zentral ist die Verpflichtung zur Schaffung eines inklusiven Bildungssystems (Art 24). Welche Folgen sich aus dem Inkrafttreten der Behindertenrechtskonvention für die Rechtslage in Deutschland ergeben, wird (auch unter Juristen) kontrovers diskutiert. Der Streit hat sich vor allem an der Frage entzündet, ob die Länder zur Aufnahme von Schülerinnen und Schülern mit sonderpädagogischem Förderungsbedarf in eine Regelschule verpflichtet sind.

\section{Von der Integration zur Inklusion}

Im Hinblick auf die Anforderungen für die Umsetzung ist das Konzept der Inklusion radikaler und weiter gehend als das der Integration. Während die Integration auf die Wiedereingliederung behinderter Menschen in bestehende gesellschaftliche Strukturen zielt, weist das Konzept der Inklusion auf die Umgestaltung der sozialen Umwelt als Voraussetzung für die gemeinsame Nutzung und die gesellschaftliche Teilhabe durch heterogene Gruppen von Menschen - hier der Kinder und Jugendlichen - hin ${ }^{16}$. Zu Recht ist deshalb die amtliche deutsche Fassung der Konvention kritisiert worden, die den Begriff „Inklusion“ mit „Integration“ übersetzt und im Ergebnis die so definierten Anforderungen der Konvention für (weitgehend) erfüllt ansieht ${ }^{17}$.

\section{Das Konzept der Inklusion als Auftrag für alle Leistungssysteme}

Das Paradigma der Inklusion hat aber nicht nur Auswirkungen auf das Verhältnis zwischen Jugend- und Sozialhilfe. Es bedeutet, dass alle Leistungssysteme sich so verändern müssen, dass sie eine individuelle Förderung aller Personen ermöglichen - unabhängig von der Art ihrer Beeinträchtigung bzw. ihres Handicaps. Verbunden damit ist die Forderung nach der Abschaffung von Parallelstrukturen und Sondereinrichtungen. Dies kann und darf aber nicht durch eine bloße Schließung der Sondereinrichtungen und

16 Dannenbeck, Vom Integrations- zum Inklusionsparadigma, Gemeinsam Leben 2008, S. 195 ff.

17 Degener (Fußn. 15); Zinke (Fußn. 14). 
die Aufnahme der Kinder und Jugendlichen mit Behinderungen bzw. mit Beeinträchtigungen in die Regeleinrichtungen geschehen. Die große Herausforderung wird darin bestehen, das bzw. die Regelsysteme qualitativ so umzugestalten, dass die Bedarfe aller Personen abgedeckt werden. Am Ende wird die Frage zu beantworten sein, ob es noch eine Existenzberechtigung für ein Sonderrecht, für spezifische Rechtsvorschriften zur Teilhabe bzw. Eingliederung nach dem Muster des SGB IX geben kann oder ob dieses Ziel nicht bereichsspezifisch in den einzelnen Leistungsgesetzen umgesetzt, also dort inkludiert werden muss.

\section{Inklusion: Kinder- und Jugendhilfe neu denken?}

\section{Die inklusive Ausgestaltung des Leistungssystems}

Nimmt man die Botschaft der UN-Behindertenrechtskonvention ernst, so geht es also nicht länger „nur“ um die Umsetzung der sogenannten großen Lösung, nämlich eine Verlagerung des Leistungsbereichs der Eingliederungshilfe für junge Menschen mit einer körperlichen oder geistigen Behinderung aus dem SGB XII in das SGB VIII, sondern um eine neue Architektur der Kinder- und Jugendhilfe, nämlich um eine inklusive Ausgestaltung des Leistungssystems.

Dies verlangt, den gesamten Leistungskatalog des SGB VIII auf den Prüfstand zu stellen und sich Gewissheit zu verschaffen, ob alle Leistungsangebote auch die Anforderungen und Bedürfnisse der jungen Menschen mit Behinderung mit im Blick haben. Dabei wird zwischen Leistungen (der Kinder- und Jugendhilfe) zu unterscheiden sein, die als Regelleistungen einzustufen sind bzw. sich an Gruppen von Kindern und Jugendlichen richten - wie etwa Kindertagesstätten oder Jugendarbeit -, und solchen, die spezifische Bedarfe im Einzelfall decken sollen.

Im ersten Fall werden die Strukturen der Einrichtungen so umzugestalten sein, dass dort die unterschiedlichen Bedarfe aller Kinder der jeweiligen Altersgruppe gedeckt werden können. Die große Herausforderung wird (wie in der Schule) darin bestehen, auch in einem ,inklusiveren“ System unter größtmöglichem Verzicht auf besondere Einrichtungen und Dienste für spezielle Gruppen dennoch jedem jungen Menschen mit seinem individuellen Bildungs-, Betreuungs- und Förderbedarf gerecht zu werden. Es würde jedenfalls nicht ausreichen, einfach alle Kinder in einer Einrichtung zu betreuen. Wenn nämlich nicht durch entsprechende (sonder-)pädagogische Qualifizierung und Aufstockung des Personals und einer diversity-sensiblen Strukturierung der Angebote sichergestellt werden könnte, dass Kinder und Jugendliche mit festgestellten Beeinträchtigungen oder sonstigen „Eigenheiten“ (Sprachproblemen, Verhaltensauffälligkeiten, sog. ADHS etc.) eine bestmögliche individuelle Förderung erhalten, dann könnte sich das ambitionierte Konzept der Inklusion in sein Gegenteil verwandeln. Kinder mit 
speziellen Förderbedarfen würden gewissermaßen in die Rolle von inkludierten Außenseitern gedrängt. In der Phase des Übergangs zu inklusiven Angeboten müssen daher Angebotslücken vermieden werden. Keinesfalls darf es aufgrund inklusiver Angebote zu Leistungsverschlechterungen für diejenigen kommen, die bislang in Sondersystemen betreut werden.

Besonderer Aufmerksamkeit bedarf das Verhältnis der Hilfe zur Erziehung ( $\$ 27$ SGB VIII) zur Eingliederungshilfe für Kinder und Jugendliche mit Behinderungen (§ 35a SGB VIII bzw. §53 SGB XII). Hier bedarf es eines neuen Konzepts, das über die Lösung des $\S 35 \mathrm{a}$ Abs. 4 SGB VIII hinausgeht, der eine Leistungserbringung ,aus einer Hand" sicherstellen will. Zu entwickeln ist ein neuer Leistungstatbestand, der die bisher getrennten Hilfetypen zu einer „Hilfe zur Erziehung und Teilhabe für Kinder und Jugendliche“ zusammenführt ${ }^{18}$. In Bezug auf das einzelne Kind/den einzelnen Jugendlichen und dessen Lebenssituation ist dann jeweils zu entscheiden, welche Form der Unterstützung es/er bzw. das jeweilige familiale System braucht. Dazu können im Einzelfall auch Hilfsmittel für Kinder und Jugendliche mit Beeinträchtigungen gehören. Für eine spezielle Eingliederungshilfe als Leistungstatbestand ist dann kein Raum mehr.

\section{Leistungen für Eltern mit Behinderung}

Schließlich sollte in diesem Zusammenhang auch eine Lösung für die Zuordnung der Hilfebedarfe von Eltern(teilen) mit Behinderung gefunden werden. So sehr gute Gründe dafür sprechen, die Wahrnehmung von Elternaufgaben als Dimension der Teilhabe der Person anzusehen und deshalb Elternassistenz als Aufgabe der Eingliederungshilfe für den Elternteil zu qualifizieren ${ }^{19}$, so überzeugend scheint auch ein systemischer Ansatz, der alle Beeinträchtigungen des Eltern-Kind-Verhältnisses - egal wo sie verortet sind dem System der Kinder- und Jugendhilfe zuweist.

Wesentliche Voraussetzungen für die Realisierung eines solchen inklusiven Modells der Kinder- und Jugendhilfe mit all seinen Facetten sind die Qualifizierung der Fachkräfte und ihre interdisziplinäre Zusammenarbeit.

\section{Anforderungen an die Angebotsstruktur}

Konsequenzen ergeben sich auch für die Leistungserbringer. Sie haben sich mit den Kategorien Eingliederungshilfe und Sozialhilfe und den jeweiligen Kostenträgern ,arrangiert“". Wenn es künftig keine Spezialeinrichtungen mehr geben soll, höchstens noch eine Binnendifferenzierung, so bedarf es eines flexiblen Leistungsangebots, das sich auf unterschiedliche Bedarfe einstellt und in der Lage ist, möglichst maßgeschneiderte Hil-

18 Vorholz (Fußn. 12).

19 Bundesverwaltungsgericht, Urteil vom 22.10.2009 - 5 C 19.08 - juris Rdnr. 15. 
fen zu erbringen. Wir brauchen also eine bunte, breite, inklusive Hilfelandschaft. Sicherlich werden auch die Träger der öffentlichen Jugendhilfe gut beraten sein, mit den Einrichtungen und Diensten zusammenzuarbeiten, die bisher vertraglich in engem Kontakt zu den Trägern der Sozialhilfe standen. Dennoch kann es keine Besitzstandswahrung geben, d. h., Träger von Einrichtungen und Diensten müssen sich den neuen Anforderungen des Inklusionsparadigmas stellen.

\section{Flexible Übergänge in die Sozialhilfe}

Wenn es wegen der Altersgrenze mit 18 oder 21 Jahren zu einem Zuständigkeitswechsel von der Jugendhilfe zur Sozialhilfe kommt, dann sollte sichergestellt werden, dass damit nicht automatisch auch die Einrichtung gewechselt werden muss und junge Menschen mit Behinderung in einer Einrichtung „landen“, in der sich vor allem alte Menschen aufhalten. Die Übergänge sind fließend zu gestalten, so dass trotz eines Wechsels der Zuständigkeit und des Systems das Setting und die Umgebung für die betreffende Person zunächst einmal erhalten bleiben können. Hier bedarf es entsprechender Vereinbarungen zwischen den Leistungsträgern und den Einrichtungen und Diensten. Das System der Hilfeplanung (§36 SGB VIII), das sich in der Kinder- und Jugendhilfe bewährt hat, ist entsprechend weiterzuentwickeln und zu qualifizieren.

\section{Leistungsfähigkeit der Jugendämter}

Die Jugendämter müssen, um die neuen Aufgaben unter dem Blickwinkel des Inklusionsansatzes bewältigen zu können, personell und in der fachlichen Kompetenz viel breiter ausgestattet sein und über vielfältige Kompetenzen verfügen. Dies setzt ein bestimmtes Niveau personeller und finanzieller Leistungsfähigkeit voraus. Dieses wiederum kann - ökonomisch betrachtet - erst ab einer bestimmten Mindestgröße erwartet werden. Jugendämter mit einem Einzugsbereich von 20000 Einwohnern, wie sie in kreisangehörigen Gemeinden einzelner Bundesländer etabliert worden sind, können diese Aufgaben nicht bewältigen.

\section{Das Ende der Ausfallbürgschaft für andere Systeme}

Die Forderung nach einem inklusiven Hilfesystem richtet sich aber nicht nur an die Kinder- und Jugendhilfe, sondern an alle Leistungssysteme, also auch an die Schulen, die Arbeitsförderung, das Gesundheitssystem und die Sozialhilfe. Wenn alle Systeme die Implikationen der UN-Behindertenrechtskonvention ernst nehmen und sich auf den Weg zur Etablierung einer inklusiven Ausrichtung ihres Leistungsangebots machen, 
dann muss dies auch Folgen an den Schnittstellen haben. So muss inklusive Bildung (in der Schule) die Förderung aller Kinder im Blick haben - was etwa die Förderung von Kindern mit Lese- oder Rechtschreibschwäche bzw. auch die Bereitstellung der Assistenzdienste einschließt. Die Auslagerung solcher Formen der Sonderförderung oder Assistenzaufgaben auf die subsidiären Fürsorgesysteme, wie wir sie bisher kennen, hat damit keine Berechtigung mehr.

So wird genauer zu prüfen sein, welche Leistungen bei konsequenter Umsetzung des Inklusionsansatzes bei der Kinder- und Jugendhilfe verbleiben und welche originär vom Gesundheitssystem, vom Schulsystem, von den Trägern der Grundsicherung für Arbeitssuchende (SGB II) und von den Trägern der Leistungen der Arbeitsförderung (SGB III) zu gewähren sind. Auf die Kinder- und Jugendhilfe werden daher neben den erheblichen neuen Anforderungen auch entlastende Effekte zukommen - wenn alle Systeme ihrer Verantwortung gerecht werden!

\section{Zum Stand der (politischen) Debatte}

\section{Die Positionierung des 13. Kinder- und Jugendberichts}

Im zeitlich engen Zusammenhang mit der Konvention ist der 13. Kinder- und Jugendbericht zum Thema Kinder und Gesundheit erstellt worden, der sich allgemein und abstrakt auch mit dem Thema Behinderung befasst, jedoch keine konkreten Empfehlungen zur Neugestaltung des deutschen Rechts aufstellt ${ }^{20}$. Allerdings findet sich in der Stellungnahme der Bundesregierung ein eindeutiges Bekenntnis. Sie unterstützt nicht nur den inklusiven Ansatz der Berichtskommission, sondern auch deren Aussage, dass alle Maßnahmen an einer Inklusionsperspektive auszurichten sind, die keine Aussonderung akzeptiert. Schließlich stellt die Bundesregierung fest: „Das Leistungsangebot für behinderte Kinder und Jugendliche muss sich primär an der Lebenslage Kindheit und Jugend orientieren", benennt aber auch die Herausforderungen für die Ausgestaltung eines inklusiven Hilfesystems. Niemanden wird der Hinweis überraschen, dass sich (auch) die Sachverständigenkommission zum 14. Kinder- und Jugendbericht, der zu Beginn des Jahres 2013 mit der Stellungnahme der Bundesregierung dem Bundestag und damit auch der Fachöffentlichkeit vorgelegt worden ist, nachdrücklich für die Verwirklichung der großen Lösung ausspricht ${ }^{21}$.

20 Lüders, Der 13. Kinder- und Jugendbericht und die „Große Lösung“, Forum Erziehungshilfen 2010, S. $207 \mathrm{ff}$.

21 14. Kinder- und Jugendbericht, BT-Dr. 17/12200, S. 17, 370 f. 


\section{Der Nationale Aktionsplan zur Umsetzung der UN-Behindertenrechtskonvention}

In ihrem Nationalen Aktionsplan zur Umsetzung der UN-Behindertenrechtskonvention vom September 2011 räumt die Bundesregierung Abgrenzungs- und Schnittstellenprobleme bei der gegenwärtigen Aufgabenverteilung zwischen Sozial- und Jugendhilfe ein und verweist auf die Beratungen der gemeinsamen Arbeitsgruppe der Arbeits- und Sozialministerkonferenz (ASMK) und der Jugend- und Familienministerkonferenz (JFMK) mit dem Bund, den kommunalen Spitzenverbänden und der Bundesarbeitsgemeinschaft der überörtlichen Träger der Sozialhilfe, um am Ende folgendes Signal zu setzen:,Perspektivisch gilt es, die unterschiedliche Verantwortungsaufteilung zu überwinden und die Eingliederungshilfe für Kinder und Jugendliche mit Behinderungen unter dem Dach des SGB VIII im Konsens zwischen Bund, Ländern und Gemeinden zusammenzuführen („Große Lösung SGB VIII“)“. Inzwischen hat sich auch die gemeinsame Arbeitsgruppe mehrheitlich für die große Lösung ausgesprochen.

\section{Inklusion als gesellschaftspolitische Herausforderung}

Die Botschaft der UN-Behindertenrechtskonvention richtet sich aber nicht nur an die staatlichen Institutionen, sondern auch an die Gesellschaft insgesamt. Wenn wir unser soziales Umfeld ansehen, dann können wir feststellen, dass noch ein weiter Weg zurückzulegen ist, bis Behinderung ,als Ausdruck der Vielfalt menschlichen Lebens, als Normalität" verstanden wird. In einer Gesellschaft, die Effizienz, Schnelligkeit und Schönheit als „Maß aller Dinge“ begreift, bedarf es eines erheblichen Umdenkens, wenn nun die Kinder ehrgeiziger Eltern gemeinsam mit Kindern mit (schweren) körperlichen oder geistigen Beeinträchtigungen gefördert werden sollen. Aber auch für die Kinder mit Beeinträchtigungen wird die Erkenntnis schmerzhaft sein, wenn sie (immer wieder) feststellen müssen, dass andere schneller und fitter sind als sie selbst. Auf die Fachkräfte kommen daher enorme Herausforderungen zu, damit das Ziel, dass die gemeinsame Erziehung und Förderung für die Entwicklung aller Kinder vorteilhaft ist, auch tatsächlich erreicht werden kann.

Dies bedeutet andererseits, dass die rechtliche und fachpolitische Diskussion nur und erst dann ihr Ziel erreichen kann, wenn sie von einer gesellschaftspolitischen Diskussion begleitet wird. Hier stehen wir erst am Anfang. 
Die „große Lösung“ - eine unendliche Geschichte?

\section{Literatur}

Banafsche, Kinder und Jugendliche mit Behinderung zwischen SGB VIII und SGB XII - im Lichte der UN-Behindertenrechtskonvention, Zeitschrift für Kindschaftsrecht und Jugendhilfe (ZKJ) 2011, S. 116-123.

Bielefeldt, Zum Innovationspotenzial der Behindertenrechtskonvention, 3. Aufl. 2009 (www.institut-fuer-menschenrechte.de).

Dannenbeck, Vom Integrations- zum Inklusionsparadigma, Gemeinsam leben 2008, S. 195-202.

Degener, Die UN-Behindertenrechtskonvention als Inklusionsmotor, Recht der Jugend und des Bildungswesens 2009, S. 200-219.

Fegert, Kinder und Jugendliche mit Behinderung, Gemeinsames Sonderheft Jugendamt/ Zeitschrift für Kindschaftsrecht und Jugendhilfe 2010, S. 13-14.

Gerlach/Hinrichs, Inklusion und die „Große Lösung“ für die Jugend- und Behindertenhilfe, Zeitschrift für Kindschaftsrecht und Jugendhilfe 2011, S. 86-92, S. 130-135.

Hasenclever, Jugendhilfe und Jugendgesetzgebung seit 1900, Göttingen 1978.

Lüders, Der 13. Kinder- und Jugendbericht und die „Große Lösung“, Forum Erziehungshilfen 2010, S.207-211.

Meysen, Kinder- und Jugendhilfe an allen Schnittstellen: zentrale Anlaufstelle, Ausfallbürge, Netzeknüpfer, Gemeinsames Sonderheft Jugendamt/Zeitschrift für Kindschaftsrecht und Jugendhilfe 2010, S. 21-24.

Schwengers, Eingliederungshilfen für seelisch behinderte Kinder und Jugendliche, Stuttgart u. a. 2007.

Struck/Porr/Koch, Zeit lassen....aber ganz schnell anfangen! Zur aktuellen Debatte um die große Lösung, Forum Erziehungshilfen 2010, S. 196-201.

Vorholz, „Große Lösung“? Zur Diskussion in den kommunalen Spitzenverbänden, in: Forum Erziehungshilfen 2010, S. 216-218.

Wiesner, Eingliederungshilfe für seelisch behinderte junge Menschen als Aufgabe der Jugendhilfe, Zentralblatt für Jugendrecht 1996, S. 199-203 und S.278-282.

Wiesner, Die Bedeutung des Neunten Buches Sozialgesetzbuch - Rehabilitation und Teilhabe behinderter Menschen - für die Kinder- und Jugendhilfe, Zentralblatt für Jugendrecht 2001, S. 281-287.

Zinke, Die UN-Behindertenrechtskonvention und ihre Bedeutung für eine Inklusionsperspektive junger Menschen, Forum Erziehungshilfen 2010, S. 212-215. 


\title{
Die ,große Lösung“ als gleichheitsrechtliches Gebot - Verfassungsrechtliche Grenzen des dualen Systems der Eingliederungshilfe für Kinder und Jugendliche
}

\author{
Minou Banafsche
}

I. Einleitung

II. Die Vereinbarkeit des „dualen Systems“ mit Art. 3 GG 171

1. Prüfungsmaßstab

a) Persönlicher Anwendungsbereich des Art. 5 Abs. 2 UN-BRK 171

b) Sachlicher Anwendungsbereich des Art. 5 Abs. 2 UN-BRK 172

c) Das Verhältnis der UN-BRK zum Grundgesetz 174

d) Ergebnis 176

2. Vereinbarkeit der dualen Eingliederungshilfe mit Art. 3 GG 176

a) Gleichheitsrechtlicher Prüfungsmaßstab 177

b) Rechtlich relevante Ungleichbehandlung 179

aa) Zuständigkeitsdivergenzen 179

bb) Leistungsrechtliche Divergenzen 180

cc) Ergebnis 181

c) Verfassungsrechtliche Rechtfertigung der Ungleichbehandlung 181

aa) Zuständigkeitsdivergenzen $\quad 182$

bb) Leistungsrechtliche Divergenzen 183

cc) Ergebnis 185

3. Auflösung der Ungleichbehandlung 185

a) Vorzüge einer einheitlichen Regelung per se 186

b) Vorzüge einer einheitlichen Regelung im SGB XII 186

c) Vorzüge einer einheitlichen Regelung im SGB VIII (,große Lösung“) 187

aa) Die gesetzliche Verortung der Eingliederungshilfe im SGB XII 187

bb) Größere Sachnähe des SGB VIII 190

d) Ergebnis 191

$\begin{array}{ll}\text { III. Schluss } & 191\end{array}$

\section{Einleitung}

Das deutsche Sozialleistungsrecht ordnet Kinder und Jugendliche mit Behinderungen unterschiedlichen Regelungssystemen je nach Art ihrer Behinderung zu. Kinder und Jugendliche mit seelischen Behinderungen erhalten Eingliederungshilfe nach $\S 35 \mathrm{a}$ 
SGB VIII, Kinder und Jugendliche mit körperlichen und/oder geistigen Behinderungen erhalten bei Vorliegen aller Leistungsvoraussetzungen Eingliederungshilfe nach den $\S \S 53$ ff. SGB XII.

Unter dem Punkt „Neue Perspektiven für Kinder und Jugendliche mit Behinderung“ postuliert der 13. Kinder- und Jugendbericht Folgendes:

„Die Einnahme einer inklusiven Perspektive verlangt ein Leistungsangebot für behinderte Kinder und Jugendliche, das sich primär an der Lebenslage ,Kindheit und Jugend' orientiert und erst sekundär nach der Behinderung oder anderen Benachteiligungen und Belastungen in dieser Lebenslage differenziert. Dies ist nach Auffassung der Bundesregierung der Maßstab, an dem die Leistungen für junge Menschen mit Behinderung zu messen sind."1

Fest steht, dass diese Forderung mitnichten neu ist ${ }^{2}$. Bezweifelt werden muss, ob die bis dato vorliegenden Dokumente der maßgeblichen politischen Akteure - darunter der zitierte 13. Kinder- und Jugendbericht, der Beschluss der 86. Arbeits- und Sozialministerkonferenz (ASMK) 2009³, der Zwischenbericht der von der ASMK und Jugend- und Familienministerkonferenz (JFMK) eingesetzten Arbeitsgruppe „Inklusion von jungen Menschen mit Behinderung" unter Beteiligung von Bund, Ländern, kommunalen Spitzenverbänden, der Bundesarbeitsgemeinschaft der Landesjugendämter und der Bundesarbeitsgemeinschaft der überörtlichen Sozialhilfeträger aus dem Jahre $2011^{4}$ - tatsächlich eine „Perspektive“ ihrem ursprünglichen Wortsinn nach eröffnen, der dem lateinischen ,perspicere“ entstammt, was so viel bedeutet wie „mit dem Blick durchdringen, deutlich sehen, wahrnehmen"5.

Den Durchblick mag der eine oder andere bei der Debatte um die Eingliederungshilfe für Kinder und Jugendliche mit Behinderungen wohl eher verlieren. Zahlreich sind die in diesem Kontext sich ergebenden Probleme ${ }^{6}$, divers die Lösungsansätze ${ }^{7}$.

1 Bericht über die Lebenssituation junger Menschen und die Leistungen der Kinder- und Jugendhilfe in Deutschland - 13. Kinder- und Jugendbericht, BT-Drucks. 16/12860, S. 12.

2 Florian Gerlach/Knut Hinrichs, Inklusion und die „Große Lösung“ für die Jugend- und Behindertenhilfe (Teil 1), ZKJ 2012, S. 86.

3 Beschlussprotokoll der 86. Konferenz der Ministerinnen und Minister, Senatorinnen und Senatoren für Arbeit und Soziales der Länder, 2009, S. 11 f., abrufbar unter: http://www.sms.sachsen.de/ download/Verwaltung/ergebnis-asmk2009.pdf (letzter Zugriff: 6.5.2012).

4 Siehe das Beschlussprotokoll der 88. Konferenz der Ministerinnen und Minister, Senatorinnen und Senatoren für Arbeit und Soziales der Länder, 2011, S. 34 ff., abrufbar unter: http://www.sms. sachsen.de/download/Verwaltung/Protokoll_extern.pdf (letzter Zugriff: 5.6.2012).

5 Duden, Das Herkunftswörterbuch - Etymologie der deutschen Sprache, 4. Aufl. Mannheim 2007, S. 600.

6 Siehe etwa: Minou Banafsche, Kinder und Jugendliche mit Behinderung zwischen SGB VIII und SGB XII - im Lichte der UN-Behindertenrechtskonvention, ZKJ 2011, S. 116 ff.; Gerlach/Hinrichs (Fußn. 2), ZKJ 2012, S. 86 ff.; Zwischenbericht 2011 (Fußn. 4), S. 36 ff.

7 Eine Bewertung vornehmend: Stefan Greß/Stephan Rixen/Jürgen Wasem, Eingliederungshilfe für seelisch behinderte Kinder und Jugendliche: Abgrenzungsprobleme und Reformszenarien, VSSR 2009, S. 43, 51 ff.; ferner: Zwischenbericht 2011 (Fußn. 4), S. 24 ff., 28 ff. 
Ziel des vorliegenden Beitrags soll es sein, das „duale System“ der Eingliederungshilfe für Kinder und Jugendliche mit Behinderungen auf seine Vereinbarkeit mit dem Gleichheitssatz des Art. 3 Grundgesetz (GG) zu überprüfen.

\section{Die Vereinbarkeit des ,,dualen Systems “ mit Art. 3 GG}

\section{Prüfungsmaßstab}

Denkbar wäre als Prüfungsmaßstab neben Art. 3 GG auch das Verbot der Diskriminierung aufgrund von Behinderung aus Art. 5 Abs. 2 UN-BRK, welches den allgemeinen Grundsatz der Nichtdiskriminierung aus Art. 3 Buchstabe a UN-BRK konkretisiert. Danach verbieten die Vertragsstaaten ,jede Diskriminierung aufgrund von Behinderung [...].“ Darunter fällt gem. Art. 2 Unter-Abs. 3 UN-BRK, ,jede Unterscheidung, Ausschließung oder Beschränkung aufgrund von Behinderung, die zum Ziel oder zur Folge hat, dass das auf die Gleichberechtigung mit anderen gegründete Anerkennen, Genießen oder Ausüben aller Menschenrechte und Grundfreiheiten im politischen, wirtschaftlichen, sozialen, kulturellen, bürgerlichen oder jedem anderen Bereich beeinträchtigt oder vereitelt wird. Sie umfasst jede Form der Diskriminierung, einschließlich der Versagung angemessener Vorkehrungen." 8 Letztere werden ihrerseits in Art. 2 Unter-Abs. 4 UNBRK definiert. 9

\section{a) Persönlicher Anwendungsbereich des Art. 5 Abs. 2 UN-BRK}

Eine Definition von „Behinderung“ enthält die UN-BRK nicht, insbesondere nicht in dem eigens mit „Begriffsbestimmungen“ (,definitions“) überschriebenen Art. 2 UNBRK. 10 Allerdings eröffnet Art. 1 UN-BRK, der eigentlich den „Zweck“ (,purpose“) der Konvention zu regeln vorgibt, in Unter-Abs. 2 ihren persönlichen Anwendungsbereich. Danach zählen zu den Menschen mit Behinderungen solche, ,die langfristige körperliche, seelische, geistige oder Sinnesbeeinträchtigungen haben, welche sie in Wechselwirkung mit verschiedenen Barrieren an der vollen, wirksamen und gleichberechtigten Teilhabe an der Gesellschaft hindern können." Einen Hinweis, warum von

8 Zum Grundsatz der Nichtdiskriminierung nach der UN-BRK: Valentin Aichele/Nina Althoff, NichtDiskriminierung und angemessene Vorkehrungen in der UN-Behindertenrechtskonvention, in: Antje Welke (Hrsg.), UN-Behindertenrechtskonvention mit rechtlichen Erläuterungen, Berlin 2012, S. 104, Rdnr. 5 ff.

$9 \mathrm{Zu}$ den angemessenen Vorkehrungen nach Art. 2 Unter-Abs. 4 UN-BRK: Aichele/Althoff (Fußn. 8), S. 104, Rdnr. $41 \mathrm{ff}$.

10 Theresia Degener, Welche legislativen Herausforderungen bestehen in Bezug auf die nationale Implementierung der UN-Behindertenrechtskonvention in Bund und Ländern?, br 2009, S. 34. 
einer Definition von Behinderung abgesehen wurde, gibt Buchstabe e der Präambel, dem die Erkenntnis zugrunde liegt, dass sich das Verständnis von Behinderung ständig weiterentwickelt. ${ }^{11}$ Angesichts dessen wollte man den Schutzbereich der UN-BRK nicht durch eine statische Begriffsbestimmung verengen. ${ }^{12}$

Da Art. 1 Unter-Abs. 2 UN-BRK weiter reicht als die Definition des $\S 2$ Abs. 1 Satz 1 SGB IX ${ }^{13}$, auf den wiederum die im Falle von Kindern und Jugendlichen mit körperlichen und/oder geistigen Behinderungen maßgebliche Vorschrift des $\S 53$ Abs. 1 Satz 1 SGB XII verweist ${ }^{14}$ und auf den die den Anspruch auf Eingliederungshilfe für Kinder und Jugendliche mit seelischen Behinderungen gewährende Norm des § 35a Abs. 1 Satz 1 SGB VIII mittelbar rekurriert ${ }^{15}$, ist der persönliche Anwendungsbereich der UN-BRK vorliegend eröffnet.

\section{b) Sachlicher Anwendungsbereich des Art. 5 Abs. 2 UN-BRK}

Die UN-BRK unterscheidet nicht zwischen Behinderungsarten, sondern bezieht alle unter Art. 1 Unter-Abs. 2 fallenden Menschen gleichermaßen in ihren Schutzbereich ein. Zweck des Übereinkommens ist es gem. Art. 1 Unter-Abs. 1, „den vollen und

11 Gem. Art. 31 Abs. 2 Wiener Vertragsrechtskonvention (WVK) ist die Präambel eines völkerrechtlichen Vertrages für dessen Auslegung neben dem Vertragswortlaut, Zusammenhang sowie Sinn und Zweck des Vertrages ebenfalls von Bedeutung. Vgl. auch: Manfred Nowak, U.N. Covenant on Civil and Political Rights - CCPR Commentary, 2. Aufl. Kehl am Rhein 2005, Präambel Rdnr. 1.

12 Dazu: Theresia Degener, Die UN-Behindertenrechtskonvention als Inklusionsmotor, RdJB 2009, S. 200, 204; Theresia Degener, Die UN-Behindertenrechtskonvention - Grundlage für eine neue inklusive Menschenrechtstheorie, VN 2010, S. 57 f.; zu dem Streitstand während des Entstehungsprozesses der Konvention: Theresia Degener, Menschenrechtsschutz für behinderte Menschen - Vom Entstehen einer neuen Menschenrechtskonvention der Vereinten Nationen, VN 2006, S. 104, 106; Jochen von Bernstorff, Menschenrechte und Betroffenenrepräsentation: Entstehung und Inhalt eines UN-Antidiskriminierungsübereinkommens über die Rechte von behinderten Menschen, ZaöRV 67 (2007), S. 1041, 1047.

13 Siehe die Gegenüberstellung von Art. 1 Unter-Abs. 2 UN-BRK und $\S 2$ Abs. 1 SGB IX bei: Minou Banafsche, Die UN-Behindertenrechtskonvention und das deutsche Sozialrecht - eine Vereinbarkeitsanalyse anhand ausgewählter Beispiele (Teil I), SGb 2012, S. 373, 374 ff.

14 Einen eigenständigen, von $\S 2$ Abs. 1 Satz 1 SGB IX abweichenden Behinderungsbegriff implementiert $\S 53$ Abs. 1 Satz 1 SGB XII daher nicht. Siehe: Felix Welti, in: Klaus Lachwitz/Walter Schellhorn/ders. (Hrsg.), HK-SGB IX, 3. Aufl. Köln 2010, § 2 Rdnr. 10; Eckart Stevens-Bartol, in: Werner Feldes/Wolfhard Kohte/ders. (Hrsg.), SGB IX, Frankfurt am Main 2009, 2 Rdnr. 5; Bernhard Knittel, SGB IX, 6. Aufl. Köln 2012, § 2 Rdnr. 9; Hartmut Haines, in: Dirk Dau/Franz Josef Düwell/Jacob Joussen (Hrsg.), LPK-SGB IX, 3. Aufl. Baden-Baden 2011, § 2 Rdnr. 15; von einem einheitlichen Behinderungsbegriff des $\S 2$ Abs. 1 Satz 1 SGB IX gehen ebenfalls aus: Volker Wahrendorf, in: Christian Grube/ders. (Hrsg.), SGB XII, 4. Aufl. München 2012, § 53 Rdnr. 13; Renate Bieritz-Harder, in: dies./Wolfgang Conradis/Stephan Thie (Hrsg.), LPK-SGB XII, 9. Aufl. Baden-Baden 2012, § 53 Rdnr. 3 ff., 11.

15 Vgl. Thomas Meysen, in: Johannes Münder/ders./Thomas Trenczek (Hrsg.), Frankfurter Kommentar zum SGB VIII, 6. Aufl. Baden-Baden 2009, § 35a Rdnr. 6, 16; Reinhard Wiesner, in: ders. (Hrsg.), SGB VIII, 4. Aufl. München 2011, § 35a Rdnr. 3, 5 f. 
gleichberechtigten Genuss aller Menschenrechte und Grundfreiheiten durch alle Menschen mit Behinderungen zu fördern, zu schützen und zu gewährleisten und die Achtung der ihnen innewohnenden Würde zu fördern." Verboten ist demgemäß nach Art. 5 Abs. 2 UN-BRK, ,jede Diskriminierung aufgrund von Behinderung“. Die Passus „,durch alle Menschen mit Behinderungen“" und ,jede Diskriminierung aufgrund von Behinderung" erlauben indes nicht nur eine Blickrichtung, die die Gruppe der Menschen mit Behinderungen der Gruppe der Menschen ohne Behinderungen gegenüberstellt, sondern zudem eine solche, die ungerechtfertigte Unterscheidungen innerhalb der Gruppe der Menschen mit Behinderungen verbietet.

Dies gilt umso mehr, als „,die Achtung vor der Unterschiedlichkeit von Menschen mit Behinderungen“ (,respect for difference“) im Kontext mit der „,menschlichen Vielfalt“ (,human diversity“) die Individualität von Menschen mit Behinderungen zum allgemeinen Grundsatz der Konvention erhebt (vgl. Art. 3 Buchstabe d UN-BRK, Buchstabe i der Präambel). Dem wird auch durch die teilweise erfolgte Bezugnahme auf bestimmte Beeinträchtigungen in einzelnen Vorschriften Rechnung getragen, zum Beispiel in Art. 9 Abs. 2 UN-BRK, der in öffentlichen Gebäuden explizit Beschilderungen in Brailleschrift (Buchstabe d) oder das Zurverfügungstellen von Gebärdendolmetschern verlangt (Buchstabe e), um Zugangsbarrieren abzubauen oder nicht zur Entstehung gelangen zu lassen, oder in Art. 24 Abs. 3 Satz 2 Buchstabe c UN-BRK, der zu geeigneten Maßnahmen gegenüber blinden, gehörlosen oder taubblinden Menschen verpflichtet, um diesen eine bestmögliche schulische und soziale Entwicklung zu ermöglichen. Gemäß dem Buchstaben h der Präambel liegt dem Übereinkommen die Erkenntnis zugrunde, „dass jede Diskriminierung aufgrund von Behinderung eine Verletzung der Würde und des Wertes darstellt, die jedem Menschen innewohnen [...].“ Der Schutz vor Diskriminierungen von Menschen mit Behinderungen vermittelt somit einen über einen bloß gruppenbezogenen Schutz hinausgehenden Individualschutz.

Wenn demnach der sich als Synthese der Maßgaben des Art. 1 Unter-Abs. 1 und des Art. 5 Abs. 2 UN-BRK darstellende Art. 4 Abs. 1 Satz 1 UN-BRK den Vertragsstaaten auferlegt, „die volle Verwirklichung aller Menschenrechte und Grundfreiheiten für alle Menschen mit Behinderungen ohne Diskriminierung aufgrund von Behinderung zu gewährleisten und zu fördern“ und zu diesem Zweck „alle geeigneten Gesetzgebungs-, Verwaltungs- und sonstigen Maßnahmen zur Umsetzung der in diesem Übereinkommen anerkannten Rechte zu treffen“ (Art. 4 Abs. 1 Satz 2 Buchstabe a UN-BRK) ${ }^{16}$, ebenso

16 Daraus i. V. m. Art. 4 Abs. 5 UN-BRK folgt, dass Verpflichtungsadressaten Legislative, Exekutive und Judikative auf den Ebenen von Bund, Ländern und Kommunen gleichermaßen sind. Freilich ergibt sich dies letztlich schon aus Art. 20 Abs. 3 GG, wonach die Gesetzgebung an die verfassungsmäßige Ordnung gebunden ist und vollziehende Gewalt und Rechtsprechung an Gesetz und Recht gebunden sind. Vgl. zum Ganzen auch: Valentin Aichele, Die UN-Behindertenrechtskonvention und ihr Fakultativprotokoll - Ein Beitrag zur Ratifikationsdebatte, Policy Paper No. 9, Berlin 2008, S. 8; Valentin Aichele, Behinderung und Menschenrechte: Die UN-Konvention über die Rechte von Menschen mit Behinderungen, APuZ 23/2010, S. 13, 17; zu der Rolle der Kommunen 
„zur Änderung oder Aufhebung bestehender Gesetze, Verordnungen, Gepflogenheiten und Praktiken [...], die eine Diskriminierung von Menschen mit Behinderungen darstellen“ (Art. 4 Abs. 1 Satz 2 Buchstabe b UN-BRK), bedeutet dies die Verpflichtung, ihre Rechtsordnungen unbeschadet relationaler Erwägungen unter anderem auf solche Regelungen zu überprüfen, die an das Merkmal der Behinderung spezifische Rechtsfolgen knüpfen, um sodann ihre unter Umständen diskriminierende Wirkung zu eruieren.

\section{c) Das Verhältnis der UN-BRK zum Grundgesetz}

Vor dem Hintergrund der vorstehenden Ausführungen tangiert die unterschiedliche gesetzliche Behandlung von Kindern und Jugendlichen mit Behinderungen je nach Behinderungsart den Schutzbereich des Art. 5 Abs. 2 UN-BRK.

Gemäß dem Grundsatz der Völkerrechtsfreundlichkeit, dem die deutsche Rechtsordnung im Gesamten und mit ihr das Grundgesetz folgt ${ }^{17}$, gilt es, das einfache wie das Verfassungsrecht im Sinne der für Deutschland verbindlichen völkerrechtlichen Normen - völkerrechtskonform - zu interpretieren. ${ }^{18}$ Die Grenzen der Auslegung bilden dabei - entsprechend den Grundsätzen verfassungskonformer Auslegung ${ }^{19}$ - Wortlaut

als Träger öffentlicher Gewalt und Teil der Exekutive: BVerfGE 73, 118, 191; 83, 37, 53 f.; vgl. auch: Bodo Pieroth, in: Hans Jarass/ders., GG, 12. Aufl. München 2012, Art. 28 Rdnr. 10.

17 Die Völkerrechtsfreundlichkeit des Grundgesetzes folgt namentlich aus Art. 1 Abs. 2, Art. 24 bis 26 und 100 Abs. 2 GG. Vgl.: Karl-Peter Sommermann, Völkerrechtlich garantierte Menschenrechte als Maßstab der Verfassungskonkretisierung - Die Menschenrechtsfreundlichkeit des Grundgesetzes, AöR 114 (1989), S. 391, 417; Karl-Peter Sommermann, in: Hermann von Mangoldt/Friedrich Klein/Christian Starck (Hrsg.), Grundgesetz, Bd. 2, 6. Aufl. München 2010, Art. 20 Rdnr. 254; vgl. auch: BVerfGE 111, 307, $317 \mathrm{f}$.

18 Sommermann, in: von Mangoldt/Klein/Starck (Fußn. 17), Art. 20 Rdnr. 254; Rudolf Geiger, Grundgesetz und Völkerrecht, 5. Aufl. München 2010, § 38 II.; Albert Bleckmann, Die Völkerrechtsfreundlichkeit der deutschen Rechtsordnung, DÖV 1979, S. 309, 312 f.; Albert Bleckmann, Der Grundsatz der Völkerrechtsfreundlichkeit der deutschen Rechtsordnung, DÖV 1996, S. 137, 140 f.; Thomas Giegerich, Wirkung und Rang der EMRK in den Rechtsordnungen der Mitgliedstaaten, in: Rainer Grote/Thilo Marauhn (Hrsg.), EMRK/GG - Konkordanzkommentar zum europäischen und deutschen Grundrechtsschutz, Tübingen 2006, Kap. 2 Rdnr. 19 ff.; Robert Uerpmann, Die Europäische Menschenrechtskonvention und die deutsche Rechtsprechung - Ein Beitrag zum Thema Völkerrecht und Landesrecht, Berlin 1993, S. 109 ff.; ferner: BVerfGE 74, 358, 370; 111, 307, 317 f.; 128, 326, 366 ff.; spezifisch zur UN-BRK: BVerfGE 128, 282, 306.

$19 \mathrm{Zu}$ den Grenzen verfassungskonformer Auslegung: BVerfGE 8, 28, 34; 8, 38, 41; 18, 97, 111;54, 277, 299 f.; 71, 81, 105; 90, 263, 275; 95, 64, 93; 110, 226, 267; 118, 212, 234; 119, 247, 274; ferner: Sommermann, in: von Mangoldt/Klein/Starck (Fußn. 17), Art. 20 Rdnr. 260; Christian Starck, in: Hermann von Mangoldt/Friedrich Klein/ders. (Hrsg.), Grundgesetz, Bd. 1, 6. Aufl. München 2010, Art. 1 Rdnr. 328; Christian Hillgruber, Richterliche Rechtsfortbildung als Verfassungsproblem, JZ 1996, S. 118, 119; Andreas Voßkuhle, Theorie und Praxis der verfassungskonformen Auslegung von Gesetzen durch Fachgerichte - Kritische Bestandsaufnahme und Versuch einer Neubestimmung, AöR 125 (2000), S. 177, 193 f. 
und erkennbarer Zweck der infrage stehenden Normen. ${ }^{20} \mathrm{Zu}$ berücksichtigen ist allerdings, dass völkerrechtliche Verträge gem. Art. 59 Abs. 2 Satz 1 GG jeweils durch Bundesgesetz in deutsches Recht überführt werden ${ }^{21}$ und selbst den Rang einfachen Bundesrechts einnehmen ${ }^{22}$. Aus diesem Grund sind völkerrechtliche Gewährleistungen kein unmittelbarer verfassungsrechtlicher Prüfungsmaßstab ${ }^{23}$, „, beeinflussen jedoch die Auslegung der Grundrechte und rechtsstaatlichen Grundsätze des Grundgesetzes“ und „dienen auf der Ebene des Verfassungsrechts als Auslegungshilfen für die Bestimmung von Inhalt und Reichweite von Grundrechten und rechtsstaatlichen Grundsätzen des Grundgesetzes, sofern dies nicht zu einer selbst nicht gewollten ${ }^{24}[\ldots]$ Einschränkung oder Minderung des Grundrechtsschutzes nach dem Grundgesetz führt [...]“"25 Das Grundgesetz ist, wie es das Bundesverfassungsgericht (BVerfG) formuliert, „nicht die weitesten Schritte der Öffnung für völkerrechtliche Bindungen gegangen“, zum einen mit Blick auf die Erforderlichkeit eines Zustimmungsgesetzes nach Art. 59 Abs. 2 Satz 1 GG, um völkerrechtlichen Verträgen zur innerstaatlichen Geltung zu verhelfen, zum anderen, weil es das Völkervertragsrecht „nicht mit dem Rang des Verfassungsrechts ausgestattet“ und somit nicht „auf die in dem letzten Wort der deutschen Verfassung liegende Souveränität" verzichtet hat. ${ }^{26}$

20 Siehe etwa: Sommermann, AöR 114 (Fußn. 17), S. 391, 418; Sommermann, in: von Mangoldt/Klein/Starck (Fußn. 17), Art. 20 Rdnr. 254; vgl. auch: Bleckmann, DÖV 1979 (Fußn. 18), S. 309, 312.

21 Im Falle der UN-BRK durch das „Gesetz zu dem Übereinkommen der Vereinten Nationen vom 13. Dezember 2006 über die Rechte von Menschen mit Behinderungen sowie zu dem Fakultativprotokoll vom 13. Dezember 2006 zum Übereinkommen der Vereinten Nationen über die Rechte von Menschen mit Behinderungen“" vom 21.12.2008, BGB1. II 2008, S. 1419.

22 BVerfGE 74, 358, 370; 111, 307, 317; exemplarisch: Bernhard Kempen, in: von Mangoldt/Klein/Starck (Fußn. 17), Art. 59 Rdnr. 92. Nach der sogenannten „Vollzugslehre“ bleibt völkerrechtlichen Verträgen aber auch nach der Überführung in deutsches Recht ihr völkerrechtlicher Charakter erhalten. Dazu und zu der Abgrenzung von der sogenannten „Transformationslehre“, nach der in deutsches Recht überführte völkerrechtliche Verträge ihren völkerrechtlichen Charakter verlieren, m. w. N.: Jochen von Bernstorff, Anmerkungen zur innerstaatlichen Anwendbarkeit ratifizierter Menschenrechtsverträge: Welche Rechtswirkungen erzeugt das Menschenrecht auf inklusive Schulbildung aus der UN-Behindertenrechtskonvention im deutschen Sozial- und Bildungsrecht?, RdJB 2011, S. 203, 204 ff.

23 BVerfGE 10, 271, 274; 34, 384, 395; 41, 126, 149; 64, 135, 157; 74, 102, 128; 111, 307, 317.

24 Siehe Art. 4 Abs. 4 Satz 2 UN-BRK, wonach ,die in einem Vertragsstaat durch Gesetze, Übereinkommen, Verordnungen oder durch Gewohnheitsrecht anerkannten oder bestehenden Menschenrechte und Grundfreiheiten [...] nicht unter dem Vorwand beschränkt oder außer Kraft gesetzt werden [dürfen], dass dieses Übereinkommen derartige Rechte oder Freiheiten nicht oder nur in einem geringeren Ausmaß anerkenne.“

25 BVerfGE 111, 307, 317; in der Sache ebenso: BVerfGE 74, 358, 370.

26 BVerfGE 111, 307, $318 \mathrm{f}$. 


\section{d) Ergebnis}

Aus den genannten Gründen, ihr Verhältnis zum Grundgesetz betreffend, soll vorliegend nicht die UN-BRK, sondern eben jenes als Prüfungsmaßstab herangezogen werden. Hierbei wird indes zu berücksichtigen sein, dass die verfassungsrechtlichen Gewährleistungen - „im Rahmen des demokratischen und rechtsstaatlichen Systems des Grundgesetzes“27 - im Lichte der UN-BRK zu interpretieren sein werden. ${ }^{28}$

\section{Vereinbarkeit der dualen Eingliederungshilfe mit Art. $3 G G$}

Gleichheitsrechtlicher Anknüpfungspunkt für die unterschiedliche gesetzliche Behandlung von Kindern und Jugendlichen mit Behinderungen je nach Behinderungsart könnte sowohl der allgemeine Gleichheitssatz des Art. 3 Abs. 1 GG als auch der besondere Gleichheitssatz des Art. 3 Abs. 3 Satz 2 GG sein, wonach niemand wegen seiner Behinderung benachteiligt werden darf. ${ }^{29}$ Der maßgebliche Unterschied liegt in den höheren Anforderungen des Art. 3 Abs. 3 Satz 2 GG an eine verfassungsrechtliche Rechtfertigung der Ungleichbehandlung. ${ }^{30}$

27 BVerfGE 111, 307, 318.

28 Diese Form der gegenseitigen Beeinflussung von Grundgesetz und Völkervertragsrecht ähnelt strukturell der sogenannten „Wechselwirkungstheorie“ von Freiheitsrechten aus Art 5 Abs. 1 GG und den allgemeinen Gesetzen nach Art. 5 Abs. 2 GG als Grundrechtsschranken. So ist „,die gegenseitige Beziehung zwischen Grundrecht und ,allgemeinem Gesetz' $[\ldots]$ nicht als einseitige Beschränkung der Geltungskraft des Grundrechts durch die ,allgemeinen Gesetze' aufzufassen; es findet vielmehr eine Wechselwirkung in dem Sinne statt, daß die ,allgemeinen Gesetze‘ zwar dem Wortlaut nach dem Grundrecht Schranken setzen, ihrerseits aber aus der Erkenntnis der wertsetzenden Bedeutung dieses Grundrechts im freiheitlichen demokratischen Staat ausgelegt und so in ihrer das Grundrecht begrenzenden Wirkung selbst wieder eingeschränkt werden müssen“, BVerfGE 7, 198, 208 f.; ferner: BVerfGE 59, 231, 265; 60, 234, 240; 61, 1, 10 f.; 66, 116, 150; 71, 206, 214; von dem Erfordernis eines „Abwägungsprozess[es] am Maßstab des Verhältnismäßigkeitsgrundsatzes“ im Rahmen der Wechselwirkungslehre spricht: Klaus Stern, Das Staatsrecht der Bundesrepublik Deutschland, Bd. IV/1, München 2006, § 108, IV 7 (S. 1476); ferner: Hans Jarass, in: ders./Pieroth (Fußn. 16), Art. 5 Rdnr. 57; Herbert Bethge, in: Michael Sachs (Hrsg.), Grundgesetz, 6. Aufl. München 2011, Art. 5 Rdnr. 146.

29 Zur Verantwortung des Staates in diesem Kontext: Hans Friedrich Zacher, Der soziale Rechtsstaat in der Verantwortung für Menschen mit Behinderungen, in: Ulrich Becker/Franz Ruland (Hrsg.), Abhandlungen zum Sozialrecht II, Heidelberg 2008, S. 175 ff.

30 Dazu unten II. 2. c). 


\section{a) Gleichheitsrechtlicher Prüfungsmaßstab}

Art. 3 Abs. 3 Satz 2 GG verbietet eine Benachteiligung „,wegen“ einer Behinderung, mithin eine Benachteiligung, die an das Merkmal der Behinderung anknüpft. ${ }^{31}$ Das Benachteiligungsverbot des Art. 3 Abs. 3 Satz 2 GG ist somit einschlägig, wenn eine Regelung eine bestimmte Rechtsfolge pauschal an den Tatbestand der „Behinderung“"32 oder an eine bestimmte Art von Behinderung knüpft, wenn die Vergleichsgruppe Menschen ohne Behinderungen sind 33 .

In der vorliegenden Konstellation der dualen Eingliederungshilfe für Kinder und Jugendliche mit Behinderungen werden die Ansprüche auf Eingliederungshilfe jeweils an eine Behinderungsart geknüpft, allerdings ist die maßgebliche Vergleichsgruppe nicht die der Menschen ohne Behinderungen, sondern die der Menschen mit einer anderen Behinderungsart. Überwiegend wird in diesem Zusammenhang eine Benachteiligung wegen einer Behinderung mit dem Argument abgelehnt, es handele sich nur um eine Differenzierung innerhalb der Gruppe von Menschen mit Behinderungen ${ }^{34}$, und auf Art. 3 Abs. 1 GG zurückgegriffen ${ }^{35}$. Dem liegt offenbar der gedankliche Automatismus zugrunde, es gehe dem besonderen Gleichheitssatz darum, die als Einheit betrachtete Gruppe der „Behinderten“ gegenüber den „Nichtbehinderten“ zu stärken.

Zwar mögen diese Erwägungen bei der Schaffung des Art. 3 Abs. 3 Satz 2 GG im Vordergrund gestanden haben, ein zwingender Gegenschluss, dass Benachteiligungen innerhalb der Gruppe von Menschen mit Behinderungen per se aus dem Schutzbereich des Art. 3 Abs. 3 Satz 2 GG ausgeschlossen sind, lässt sich dem indes nicht entnehmen. ${ }^{36}$ Beschlussempfehlung und Bericht des Rechtsausschusses zu dem „Entwurf eines Gesetzes zur Änderung des Grundgesetzes der Fraktionen der CDU/CSU, SPD und F.D.P.“37 enthalten keine Ausführungen zu einer Beschränkung des Art. 3 Abs. 3 Satz 2 GG auf das Verhältnis zwischen Menschen mit Behinderungen und Menschen ohne Behinderungen. Im Vordergrund stand das Ziel, gesellschaftliche und rechtliche Aus-

31 Guy Beaucamp, Das Behindertengrundrecht (Art. 3 Abs. 3 Satz 2 GG) im System der Grundrechtsdogmatik, DVB1. 2002, S. 997, 998; Felix Welti, Behinderung und Rehabilitation im sozialen Rechtsstaat, Tübingen 2005, S. 446 f.; Volker Neumann, in: Olaf Deinert/ders. (Hrsg.), Rehabilitation und Teilhabe behinderter Menschen - Handbuch SGB IX, 2. Aufl. Baden-Baden 2009, § 2 Rdnr. 24.

32 Beispielhaft: Welti (Fußn. 31), S. 467.

33 Lerke Osterloh, in: Sachs (Fußn. 28), Art. 3 Rdnr. 311.

34 So etwa: Rupert Scholz, in: Theodor Maunz/Günter Dürig (Begr.), Grundgesetz, Bd. I, 64. Ergänzungslieferung, Stand: Januar 2012, München 2012, Art. 3 Rdnr. 174 (Fußn. 1); Osterloh, in: Sachs (Fußn. 28), Art. 3 Rdnr. 311.

35 Siehe: Neumann, in: Deinert/ders. (Fußn. 31), § 2 Rdnr. 20, der Art. 3 Abs. 1 GG für den „flexiblere[n] Prüfungsmaßstab" erachtet.

36 Dazu, dass Gegenschlüsse nicht per se zwingend sind: Ulrich Klug, Juristische Logik, 4. Aufl. Berlin 1982, S. 137 ff.; Karl Larenz, Methodenlehre der Rechtswissenschaft, 5. Aufl. Berlin 1983, S. 374.

37 BT-Drucks. 12/6633. 
grenzungen von Menschen mit Behinderungen zu verhindern. ${ }^{38}$ Eine darüber hinausgehende Einigkeit zwischen den verschiedenen Fraktionen im Detail bestand hingegen nicht. ${ }^{39}$ Dass dabei von der „Gruppe der Behinderten“ die Rede war, belegt nicht, dass diese per se als geschlossene Gruppe betrachtet wurde, sondern nur, dass alle Menschen mit Behinderungen an dem besonderen Diskriminierungsschutz partizipieren sollten. Als Ausdruck der Wertordnung des Grundgesetzes verstärke aus Sicht der Fraktion der SPD ,das Benachteiligungs[verbot] den sozialstaatlichen Auftrag, die Voraussetzungen grundrechtlicher Freiheit und eines menschenwürdigen Daseins zu sichern und auf eine gleichberechtigte Teilhabe behinderter Menschen in der Gesellschaft hinzuwirken." 40 Die Menschenwürde ist aber nun, unbeschadet der Schwierigkeit einer Schutzbereichsbestimmung ${ }^{41}$, stets subjektbezogen ${ }^{42}$ : So wird sie zum Teil als naturgegebener Eigenwert eines jeden Menschen begriffen, der ihm qua Menschsein zukommt ${ }^{43}$, zum Teil als erst zu erreichendes Ergebnis von Selbstdarstellung in der Kommunikation mit anderen ${ }^{44}$. Das BVerfG erachtet es ,ausgehend von der Vorstellung des Grundgesetzgebers, dass es zum Wesen des Menschen gehört, in Freiheit sich selbst zu bestimmen und sich frei zu entfalten, und dass der Einzelne verlangen kann, in der Gemeinschaft grundsätzlich als gleichberechtigtes Glied mit Eigenwert anerkannt zu werden“, für durch die Verpflichtung zur Achtung und zum Schutz der Menschenwürde generell ausgeschlossen, ,den Menschen zum bloßen Objekt des Staates zu machen [...]. Schlechthin verboten ist damit jede Behandlung des Menschen durch die öffentliche Gewalt, die dessen Subjektqualität, seinen Status als Rechtssubjekt, grundsätzlich in Frage stellt [...], indem sie die Achtung des Wertes vermissen lässt, der jedem Menschen um seiner selbst willen, kraft seines Personseins, zukommt [...].“45

38 BT-Drucks. 12/8165, S. 28.

39 Vgl.: BT-Drucks. 12/8165, S. 29.

40 BT-Drucks. 12/8165, S. 29.

41 Zusammenfassend: Bodo Pieroth/Bernhard Schlink, Grundrechte - Staatsrecht II, 28. Aufl. Heidelberg 2012, Rdnr. $368 \mathrm{ff}$.

42 Von dem „Prinzip der Personhaftigkeit des Menschen“ spricht: Hasso Hofmann, Die versprochene Menschenwürde, AöR 1993, S. 353, 358, 364.

43 In der Sache bejahend, allerdings den Gemeinschaftsbezug des Menschen hervorhebend, um nicht Gefahr zu laufen, den Satz von der Würde der menschlichen Persönlichkeit ,zur magna charta des Individualismus zu machen“: Günter Dürig, Die Menschenauffassung des Grundgesetzes, JR 1952, S. 259, 261; den Eigenwert des Menschen herausstellend: Hans Carl Nipperdey, Die Würde des Menschen, in: Franz Neumann/ders./Ulrich Scheuner (Hrsg.), Die Grundrechte - Handbuch der Theorie und Praxis der Grundrechte, Bd. II, Berlin 1954, S. 1 ff., der Art. 1 Abs. 1 GG als ,naturrechtliches Elementarprinzip“ begreift; Starck, in: von Mangoldt/Klein/ders. (Fußn. 19), Art. 1 Rdnr. 11; kritisch gegenüber einer überpositiven Deutung der Menschenwürdegarantie: Matthias Herdegen, in: Maunz/Dürig (Fußn. 34), Art. 1 Rdnr. 19 f.

44 Niklas Luhmann, Grundrechte als Institution - Ein Beitrag zur politischen Soziologie, 2. Aufl. Berlin 1974, S. $68 \mathrm{f}$.

45 BVerfGE 115, 118, 153; vgl. ferner: BVerfGE 45, 187, 227 f.; 87, 209, 228; 96, 375, 399 f.; 109, 279, 312 f.; vgl. bereits: BVerfGE 30, 1, 26, wo das BVerfG seine „Objektformel“ erweitert hat, die 
Dafür, dass Art. 3 Abs. 3 Satz 2 GG weniger als kollektiv denn als individuell ausgerichtetes Recht zu begreifen ist, spricht auch der Wortlaut, ausweislich dessen niemand wegen „seiner“ Behinderung benachteiligt werden darf. ${ }^{46}$

Gestützt wird dieses Ergebnis schließlich durch die zu Art. 5 Abs. 2 UN-BRK gemachten Ausführungen ${ }^{47}$, die in die Auslegung des Grundrechts aus Art. 3 Abs. 3 Satz 2 GG im Wege völkerrechtskonformer Interpretation 48 einfließen.

Als gleichheitsrechtlicher Prüfungsmaßstab wird hier folglich Art. 3 Abs. 3 Satz 2 GG herangezogen.

\section{b) Rechtlich relevante Ungleichbehandlung}

Da sich die Zuständigkeit und die Voraussetzungen für die Leistungen zur Teilhabe gem. § 7 Satz 2 SGB IX, § 53 Abs. 4 Satz 2 SGB XII nach den für den jeweiligen Rehabilitationsträger geltenden Leistungsgesetzen richten, wirkt sich die gesetzliche Aufteilung von Kindern und Jugendlichen mit Behinderungen auf zwei Sozialleistungssysteme rechtlich vor allem in den Bereichen des Leistungsrechts und der Zuständigkeit aus. 49

\section{aa) Zuständigkeitsdivergenzen}

Zuständig für die Eingliederungshilfe nach dem SGB VIII sind mangels Zuständigkeit der überörtlichen Träger gem. $§ 85$ Abs. 2 SGB VIII ${ }^{50}$ die örtlichen Träger der Jugendhilfe ( $\$ 85$ Abs. 1 SGB VIII). Die Träger der öffentlichen Jugendhilfe werden gem. $\S 69$ Abs. 1 SGB VIII durch Landesrecht bestimmt.

In der Sozialhilfe sind gem. §97 Abs. 1 SGB XII ebenfalls die örtlichen Träger gem. § 3 Abs. 2 Satz 1 SGB XII sind dies vorbehaltlich anderslautender landesrechtlicher Bestimmungen die Kreise und kreisfreien Städte - sachlich zuständig, soweit nicht die überörtlichen Träger sachlich zuständig sind. Allerdings wird gem. § 97 Abs. 2 Satz 1 SGB XII die sachliche Zuständigkeit des überörtlichen Trägers durch Landesrecht bestimmt wie gem. § 3 Abs. 3 SGB XII auch, wer überörtlicher Träger der Sozialhilfe

zunächst allein auf das Verbot einer Objektivation des Menschen rekurrierte. Siehe etwa: BVerfGE $27,1,6$.

46 Welti (Fußn. 31), S. 470; zur Heterogenität von Gruppen und Individualität von „Gruppenrechten“: Rainer Nickel, Gleichheit und Differenz in der vielfältigen Republik - Plädoyer für ein erweitertes Antidiskriminierungsrecht, Baden-Baden 1999, S. 63 f.

47 Siehe II.1.b).

48 Dazu oben II.1.c).

49 Siehe dazu bereits: Banafsche (Fußn. 6), ZKJ 2011, S. 116, 119 f., 121 f.; Minou Banafsche, Die UN-Behindertenrechtskonvention und das deutsche Sozialrecht - eine Vereinbarkeitsanalyse anhand ausgewählter Beispiele (Teil II), SGb 2012, S. 440, 443.

50 Ihnen obliegt im Rahmen der Eingliederungshilfe für seelisch behinderte Kinder und Jugendliche gem. $\S 85$ Abs. 2 Nr. 2 SGB VIII eine fördernde Funktion. Vgl. auch: Gila Schindler, in: Münder/Meysen/Trenczek (Fußn. 15), § 85 Rdnr. 3; Wiesner, in: ders. (Fußn. 15), § 85 Rdnr. 12 ff. 
ist. Dies hat im Rahmen der Eingliederungshilfe nach dem SGB XII innerhalb einiger Länder 51 eine geteilte Zuständigkeit für ambulante und stationäre Leistungen hervorgebracht.

\section{bb) Leistungsrechtliche Divergenzen}

Während § 35a Abs. 1 Satz 1 SGB VIII für die Gewährung eines Anspruchs auf Eingliederungshilfe allein das Vorliegen einer seelischen Behinderung verlangt, erhalten Leistungen der Eingliederungshilfe auf Grundlage des §53 Abs. 1 Satz 1 SGB XII nur Personen, die durch eine Behinderung i. S. d. § 2 Abs. 1 Satz 1 SGB IX ,wesentlich in ihrer Fähigkeit, an der Gesellschaft teilzuhaben, eingeschränkt oder von einer solchen wesentlichen Behinderung bedroht sind [...], wenn und solange nach der Besonderheit des Einzelfalles, insbesondere nach Art oder Schwere der Behinderung, Aussicht besteht, dass die Aufgabe der Eingliederungshilfe erfüllt werden kann.“ Gem. § 19 Abs. 3 SGB XII werden zudem Leistungen der Eingliederungshilfe nur gewährt, „,soweit den Leistungsberechtigten [...] und, wenn sie minderjährig und unverheiratet sind, auch ihren Eltern oder einem Elternteil die Aufbringung der Mittel aus dem Einkommen und Vermögen nach den Vorschriften des Elften Kapitels dieses Buches nicht zuzumuten ist."

Der sozialhilferechtliche Anspruch des $\S 53$ Abs. 1 Satz 1 i. V. m. $§ 19$ Abs. 3 SGB XII setzt somit über das Vorliegen einer Behinderung hinaus die Bedürftigkeit des Betroffenen voraus, mithin das Unvermögen, seinen Lebensunterhalt aus eigenen Kräften zu bestreiten oder sich in der bestehenden Lebenslage selbst zu helfen ${ }^{52}$.

Des Weiteren bedarf es gem. $\S 53$ Abs. 1 Satz 1 SGB XII einer „wesentlichen“ Behinderung. Darüber, was unter einer wesentlichen körperlichen und wesentlichen geistigen Behinderung zu verstehen ist, gibt die auf Grundlage des § 60 SGB XII erlassene Eingliederungshilfe-Verordnung (EinglH-VO) in den $\S \S 1$ und 2 Aufschluss. ${ }^{53}$

Schließlich wird Eingliederungshilfe nach $\S 53$ Abs. 1 Satz 1 Halbsatz 2 SGB XII im Sinne einer Erfolgsbezogenheit nur geleistet, „,wenn und solange nach der Besonderheit

51 Namentlich sind dies Hessen, Niedersachsen, Nordrhein-Westfalen, Rheinland-Pfalz und Sachsen.

52 So: Wahrendorf, in: Grubelders. (Fußn. 14), § 9 Rdnr. 28. Auf die vom Erfordernis der Bedürftigkeit als Leistungsvoraussetzung abstrakt zu betrachtende Einschränkung der Anrechnung von Einkommen und Vermögen bei Menschen mit Behinderungen nach Maßgabe der $\S 86,87$ Abs. 1 Sätze 2 und 3 SGB XII und des $\S 92$ SGB XII, gegebenenfalls auch des $\S 92$ a SGB XII, sei an dieser Stelle hingewiesen.

53 Das Vorliegen einer wesentlichen geistigen Behinderung kann insbesondere bei Teilleistungsstörungen, wie Legasthenie und Dyskalkulie, problematisch sein: Peter Scheider, in: Walter Schellhorn/Helmut Schellhorn/Karl-Heinz Hohm (Hrsg.), SGB XII, 18. Aufl. Köln 2010, § 2 EinglH-VO Rdnr. 4; Meysen, in: Münder/ders./Trenczek (Fußn. 15), § 35a Rdnr. 40 f.; Jörg Fegert, in: Wiesner (Fußn. 15), § 35a Rdnr. 71 ff. 
des Einzelfalls, insbesondere nach Art oder Schwere der Behinderung, Aussicht besteht, dass die Aufgabe der Eingliederungshilfe erfüllt werden kann."54

\section{cc) Ergebnis}

Leistungsrechtlich ist der Erhalt von Eingliederungshilfe nach dem SGB XII demnach von weiter gehenden Voraussetzungen abhängig als im Regelungskontext des SGB VIII, so dass im Ergebnis eine rechtlich relevante Ungleichbehandlung zu bejahen ist.

Die im Rahmen der Sozialhilfe in einigen Ländern geteilte Zuständigkeit führt dazu, dass Kinder und Jugendliche mit körperlichen und/oder geistigen Behinderungen sich dort bei einem Bedarf an sowohl ambulanten als auch stationären Leistungen an unterschiedliche Leistungsträger halten müssen und auch insoweit gegenüber Kindern und Jugendlichen mit seelischen Behinderungen benachteiligt sind. Da rechtfertigungsbedürftig nur eine rechtlich relevante Ungleichbehandlung von wesentlich Gleichem ist ${ }^{55}$ und eine „Gleichbehandlung nur innerhalb des Zuständigkeitsbereichs des jeweiligen Gesetzgebers" verlangt werden kann ${ }^{56}$, beschränkt sich im Hinblick auf die Zuständigkeit das Vorliegen einer rechtlich relevanten Ungleichbehandlung von Kindern und Jugendlichen mit körperlichen und/oder geistigen Behinderungen auf die Länder mit geteilter Zuständigkeit. 57

\section{c) Verfassungsrechtliche Rechtfertigung der Ungleichbehandlung}

Eine verfassungsrechtliche Rechtfertigung der Ungleichbehandlung im Rahmen des Art. 3 Abs. 3 Satz 2 GG kann nur bejaht werden, „wenn zwingende Gründe dafür vorliegen [...]. Die nachteiligen Auswirkungen müssen unerlässlich sein, um behinderungsbezogenen Besonderheiten Rechnung zu tragen [...]."58 Es bedarf demgemäß einer strengen Verhältnismäßigkeitsprüfung ${ }^{59}$, die dem gesetzgeberischen Gestaltungsspielraum enge Grenzen setzt ${ }^{60}$.

54 Siehe zu diesem Merkmal: Wahrendorf, in: Grubelders. (Fußn. 14), § 53 Rdnr. 31; Bieritz-Harder, in: LPK-SGB XII (Fußn. 14), § 53 Rdnr. 20; VG Meiningen RdLH 1999, S. 63, 64; LSG SachsenAnhalt FEVS 57 (2006), S. 553, $554 \mathrm{f}$.

55 Pieroth/Schlink (Fußn. 41), Rdnr. 463.

56 BVerfG NVwZ 1997, S. 1207; siehe bereits: BVerfGE 10, 354, 371; 12, 139, 143; 12, 319, 324; 21, 54,68 .

57 Zum weit verstandenen Begriff des Nachteils im Rahmen des Art. 3 Abs. 3 Satz 2 GG mit weiteren Nachweisen: Osterloh, in: Sachs (Fußn. 28), Art. 3 Rdnr. 312.

58 BVerfGE 99, 341, 357; Jarass, in: ders./Pieroth (Fußn. 16), Art. 3 Rdnr. 149.

59 Osterloh, in: Sachs (Fußn. 28), Art. 3 Rdnr. 314.

60 Vgl: Pieroth/Schlink (Fußn. 41), Rdnr. 481, 488. 


\section{aa) Zuständigkeitsdivergenzen}

$\S 97$ SGB XII normiert die sachliche Zuständigkeit für die gesamte Sozialhilfe und überantwortet es dem Landesgesetzgeber zu regeln, welche Bereiche der Sozialhilfe in die sachliche Zuständigkeit der überörtlichen Träger der Sozialhilfe fallen sollen (§97 Abs. 1 Halbsatz 2 und Abs. 2 Satz 1 SGB XII) und wer überörtlicher Träger ist (§ 3 Abs. 3 SGB XII). ${ }^{61}$ Als Teil des Verwaltungsorganisationsrechts dient $\S 97$ SGB XII der Verwirklichung des materiellen Rechts. ${ }^{62}$ Nach der nach Maßgabe des Art. 30 GG statuierten bundesstaatlichen Kompetenzverteilung als für das in Art. 20 Abs. 1 und Art. 28 Abs. 1 GG verankerte Bundesstaatsprinzip ,grundlegende Vorschrift"63 führen die Länder die Sozialhilfe als eigene Angelegenheit aus (Art. 30 i. V. m. Art. 83 Halbsatz 1 GG). Gem. Art. 84 Abs. 1 Satz 1 GG regeln sie im Rahmen der Landeseigenverwaltung unter anderem die Einrichtung der Behörden 64 , was neben deren Errichtung auch die Zuweisung von Aufgaben und Befugnissen, ,insbesondere die Festlegung der Zuständigkeit für die Gesetzesausführung “65, umfasst. ${ }^{66}$ Die Regelung der sachlichen Zuständigkeit durch die Länder ist damit unmittelbarer Ausdruck und Folge des föderalen Prinzips, mithin verfassungsimmanent, und erscheint unter gleichheitsrechtlichen Gesichtspunkten nicht problematisch.

Zwar „soll“ gem. § 97 Abs. 2 Satz 2 SGB XII berücksichtigt werden, dass unter anderem für Leistungen der Eingliederungshilfe ( 8 Nr. 4 SGB XII) ,so weit wie möglich [...] eine einheitliche sachliche Zuständigkeit gegeben ist." Entgegen der durch den Begriff ,soll“ entstehenden Vermutung erfordert eine uneinheitliche Regelung der sachlichen Zuständigkeit durch den Landesgesetzgeber keinen Ausnahmefall im Sinne einer atypischen Situation 67.68 Vielmehr handelt es sich hierbei nur um eine Anregung ge-

61 Siehe bereits oben II.2.b)aa).

62 So: Wahrendorf, in: Grubelders. (Fußn. 14), § 97 Rdnr. 2.

63 BVerfGE 12, 205, 244; ferner: BVerfGE 36, 342, 365 f., wo Art. 30 GG als ,fundamentale Grundsatzbestimmung" aufgeführt wird; für das Schrifttum: Pieroth, in: Jarass/ders. (Fußn. 16), Art. 30 Rdnr. 1; Wilfried Erbguth, in: Sachs (Fußn. 28), Art. 30 Rdnr. 1; Wolfgang März, in: von Mangoldt/Klein/Starck (Fußn. 17), Art. 30 Rdnr. 1 f.

64 BVerfGE 10, 20, 48, wonach man unter einer Behörde ,im allgemeinen eine in den Organismus der Staatsgewalt eingeordnete, organisatorische Einheit von Personen und sächlichen Mitteln [versteht], die mit einer gewissen Selbständigkeit ausgestaltet dazu berufen ist, unter öffentlicher Autorität für die Erreichung der Zwecke des Staates oder von ihm geförderter Zwecke tätig zu sein.“ Dazu gehören auch die Kommunen, Kommunal- und Planungsverbände als Teil der Landesstaatsgewalt. Zur Rechtsnatur der Kommunen im Kontext mit Art. 83 GG: BVerfGE 39, 96, 109; aus dem Schrifttum: Ulrich Becker, Bayerisches Kommunalrecht, in: ders./Dirk Heckmann/Bernhard Kempen/Gerrit Manssen, Öffentliches Recht in Bayern, 5. Aufl. München 2011, 2. Teil Rdnr. 6 ff. (S. 74 f.).

65 BSGE 102, 149, 157, Rdnr. 39.

66 Vgl.: BVerfGE 105, 313, 331; BSGE 102, 149, 157, Rdnr. 39; implizit auch: BVerfGE 75, 108, $151 ; 126,77,99$.

67 Exemplarisch zu den „Soll“-Vorschriften: Hartmut Maurer, Allgemeines Verwaltungsrecht, 18. Aufl. München 2011, § 7 Rdnr. 11.

68 Bayerisches LSG, Urt. v. 31.7.2006 - L 11 SO 27/06 - Rdnr. 22 (zitiert nach juris). 
genüber dem jeweiligen Landesgesetzgeber69, der frei entscheiden kann, ob er dieser Folge leistet. ${ }^{70}$

\section{bb) Leistungsrechtliche Divergenzen}

Gegen eine verfassungsrechtliche Rechtfertigung der leistungsrechtlichen Divergenzen spricht zum einen die Normgenese des § 35a SGB VIII und sprechen zum anderen gesetzessystematische Erwägungen.

\section{(1) Die Normgenese des $\S 35$ a SGB VIII}

Die Normgenese des $\S 35$ a SGB VIII macht sichtbar, dass von Anfang an die Zuständigkeitsstreitigkeiten zwischen Jugend- und Sozialhilfe im Vordergrund standen, nicht hingegen sachliche Erwägungen für eine unterschiedliche leistungsrechtliche Behandlung von Kindern und Jugendlichen mit Behinderungen je nach Behinderungsart.

Ansprüche für Menschen mit seelischer Behinderung waren zunächst der Sozialhilfe vorbehalten; durch das Zweite Gesetz zur Änderung des Bundessozialhilfegesetzes vom 14. August 196971, gem. Art. 2 § Abs. 1 in Kraft getreten am 1. Oktober 1969, wurde dem $\S 39$ Abs. 1 Satz 1 eine Nummer 6 angefügt, die Menschen erfasste, die ,seelisch wesentlich behindert" waren. Das Gesetz für Jugendwohlfahrt (JWG) vom 11. August $1961^{72}$ enthielt demgegenüber keine spezifischen Leistungen für Kinder und Jugendliche mit Behinderungen. Allein für Minderjährige, deren „leibliche, geistige oder seelische Entwicklung gefährdet oder geschädigt" war, sah $\S 55$ JWG die Bestellung eines Erziehungsbeistands und § 62 JWG die Gewährung freiwilliger Erziehungshilfe vor, bei Gefahr im Verzug konnte das Vormundschaftsgericht gem. § 67 Abs. 1 JWG die vorläufige Fürsorgeerziehung anordnen. Störungen in der seelischen Entwicklung wurden jugendhilferechtlich somit als Erziehungsdefizit behandelt. ${ }^{73}$

Ein Diskussionsentwurf aus dem Jahre 1973 zu einem Jugendhilfegesetz (JHG) forderte die Überführung junger Menschen mit seelischer Behinderung in die Jugendhilfe und ihre dortige Verortung im Rahmen der Erziehungshilfen, um Zuständigkeitsstreitigkeiten zwischen Jugend- und Sozialhilfe zu unterbinden, die aufgrund der Abgren-

69 Begründung der Bundesregierung zum „Entwurf eines Gesetzes zur Einordnung des Sozialhilferechts in das Sozialgesetzbuch", BT-Drucks. 15/1514, S. 67.

70 Bayerisches LSG, Urt. v. 31.7.2006 - L 11 SO 27/06 - Rdnr. 22 (zitiert nach juris); Dietrich Schoch, in: LPK-SGB XII (Fußn. 14), §97 Rdnr. 12; vgl. auch: Volker Schlette, in: Karl Hauck/Wolfgang Noftz (Begr.), SGB XII, 2. Bd., 28. Lieferung, Stand: Juli 2012, Berlin 2012, § 97 Rdnr. 17, der $\S 97$ Abs. 2 SGB XII als „Blankettermächtigung des Landesgesetzgebers“ bezeichnet; ebenso: BVerwG, Beschl. v. 2.6.2008 - 5 B 188/07 - Rdnr. 6 (zitiert nach juris).

71 BGB1. I 1969, S. 1153.

72 BGBl. I 1961, S. 1206.

73 Clarita Schwengers, Eingliederungshilfen für seelisch behinderte Kinder und Jugendliche nach $\S 35$ a SGB VIII im Verhältnis zu konkurrierenden Leistungen nach dem (Sozial-)Leistungsrecht Zugleich ein Beitrag zu öffentlich-rechtlichen Ausgleichsansprüchen bei Doppelzuständigkeiten von Leistungsträgern, Stuttgart 2007, S. 43. 
zungsschwierigkeiten von seelischen Behinderungen und den Erziehungs- und Verhaltensstörungen entstanden. ${ }^{74}$ Dieser Diskussionsentwurf wurde nicht umgesetzt. ${ }^{75}$ Mit dem Gesetz zur Neuordnung des Kinder- und Jugendhilferechts (Kinder- und Jugendhilfegesetz - KJHG) vom 26. Juni 199076, gem. Art. 24 Satz 1 in Kraft getreten am 1. Januar 1991, wurden dann aber Maßnahmen der Eingliederungshilfe nach Maßgabe des $\S 40$ BSHG als Hilfen zur Erziehung in $\S 27$ Abs. 4 geregelt. 77 Die Kollisionsnorm des $\S 10$ Abs. 2 Sätze 1 und 2 (jetzt $\S 10$ Abs. 4 Sätze 1 und 2) statuierte den Vorrang des SGB VIII für Maßnahmen der Eingliederungshilfe für junge Menschen mit seelischer Behinderung vor dem BSHG. Danach waren zwar die Schwierigkeiten bei der Abgrenzung von seelischer Behinderung, die zuvor der Zuständigkeit der Sozialhilfeträger unterlegen hatte, und einem jugendhilferechtlich zu deckenden erzieherischen Bedarf behoben, allerdings entzündete sich der Streit nunmehr an der Frage, ob Eingliederungshilfe für junge Menschen mit seelischer Behinderung als Bestandteil der Erziehungshilfen auch gewährt werden konnte, wenn ein Erziehungsdefizit i. S. d. § 27 Abs. 1 SGB VIII als Grundnorm für den Rechtsanspruch auf Hilfen zur Erziehung 78 nicht vorlag; andernfalls wären die Sozialhilfeträger bei Nichterweislichkeit eines Erziehungsdefizits immer auch für Maßnahmen der Eingliederungshilfe bei seelischer Behinderung von Kindern und Jugendlichen zuständig gewesen. 79

Diesem neuerlich sich abzeichnenden Abgrenzungs- und demgemäß Zuständigkeitsproblem abzuhelfen, war schließlich die Einführung des § 35a SGB VIII als eigenständige Anspruchsnorm für Leistungen der Eingliederungshilfe bei seelischer Behinderung durch das Erste Gesetz zur Änderung des Achten Buches Sozialgesetzbuch vom 16. Februar 1993 (Art. 1 Nr. 17) ${ }^{80}$, gem. Art. 7 Abs. 1 Satz 2 des Ersten Änderungsgesetzes in Kraft getreten am 1. April 1993, zu dienen bestimmt. ${ }^{81}$

\section{(2) Die Gesetzessystematik}

Dass eine gesetzliche Verschiedenbehandlung von Kindern und Jugendlichen mit Behinderungen abhängig von der Behinderungsart nicht notwendig ist, um behinderungsbedingten Besonderheiten Rechnung zu tragen, belegt bereits der Umstand, dass $\S 35 \mathrm{a}$ Abs. 3 SGB VIII im Hinblick auf Aufgaben, Ziele und Art der Eingliederungshil-

74 Bundesministerium für Jugend, Familie und Gesundheit (Hrsg.), Diskussionsentwurf eines Jugendhilfegesetzes, Bonn 1973, S. 121 (zu § 42), S. 122 f. (zu § 42 Absatz 1), S. 128 (zu § 47 Absatz 1, Fußn. 1).

75 Schwengers (Fußn. 73), S. 44.

76 BGBl. I 1990, S. 1163.

77 Dazu die Begründung der Bundesregierung zum „Entwurf eines Gesetzes zur Neuordnung des Kinder- und Jugendhilferechts (Kinder- und Jugendhilfegesetz - KJHG)“, BT-Drucks. 11/5948, S. 53.

78 Britta Tammen/Thomas Trenczek, in: Münder/Meysen/Trenczek (Fußn. 15), § 27 Rdnr. 1; Heike Schmid-Obkirchner, in: Wiesner (Fußn. 15), § 27 Rdnr. 1.

79 Siehe: Schwengers (Fußn. 73), S. 45.

80 BGBl. I 1993, S. 239.

81 Ausführlich dazu und zu den Debatten im Kontext mit §35a SGB VIII: Schwengers (Fußn. 73), S. $45 \mathrm{ff}$. 
feleistungen keine eigenständigen Vorgaben trifft, sondern auf diejenigen des SGB XII, konkret auf $\S 53$ Abs. 3 und 4 Satz 1, §§ 54, 56 und 57 SGB XII, verweist. Dies macht im Gegenteil deutlich, dass der Gesetzgeber die differierenden leistungsrechtlichen und Zuständigkeitsnormen in einen möglichst einheitlichen Rahmen einpassen wollte, was das kompetenzrechtliche Moment als Ursache für den Dualismus ${ }^{82}$ umso stärker in den Vordergrund treten lässt.

cc) Ergebnis

Die Zuteilung von Kindern und Jugendlichen mit Behinderungen zu zwei unterschiedlichen Sozialleistungssystemen ist demnach nicht aufgrund behinderungsbezogener Besonderheiten unerlässlich, vielmehr ist die „gesetzliche Segregation“83 das Ergebnis von Zuständigkeitskonflikten zwischen den Trägern der öffentlichen Jugendhilfe und der Sozialhilfe und werden die praktischen wie rechtlichen Vorzüge einer einheitlichen Lösung von keiner Seite bestritten. Die rechtlich relevante Ungleichbehandlung ist daher nicht verfassungsrechtlich gerechtfertigt. ${ }^{84}$

\section{Auflösung der Ungleichbehandlung}

Ein Verstoß des Gesetzgebers gegen Art. 3 GG „kann [...] regelmäßig auf verschiedene Weise geheilt werden [...]." 85 Welchen Weg der Gesetzgeber hier wählt, „muss grundsätzlich der Gestaltungsfreiheit des Gesetzgebers überlassen bleiben [...].“"86

Im Folgenden sollen die zwei vorliegend im Wesentlichen ins Feld geführten Lösungsmodelle, eine einheitliche Regelung der Eingliederungshilfe für Kinder und Jugendliche mit Behinderungen im SGB XII einerseits und im SGB VIII andererseits (,große Lösung“) ${ }^{87}$, unter dem Aspekt ihrer jeweiligen Vorzüge betrachtet werden.

Die Vorteile, die eine einheitliche Regelung ungeachtet des Regelungsortes mit sich bringt, werden der Bewertung der einzelnen Modelle vorangestellt.

82 Siehe unter II.2.c).

83 Thomas Meysen, Kinder- und Jugendhilfe an allen Schnittstellen: zentrale Anlaufstelle, Ausfallbürge, Netzeknüpfer, JAmt/ZKJ Sonderheft 2012, S. 21.

84 Siehe auch die Andeutung bei: Schwengers (Fußn. 73), S. 340.

85 BVerfGE 22, 349, 361; vgl. auch: BVerfGE 103, 225, 240; ferner: Pieroth/Schlink (Fußn. 41), Rdnr. 515; Jarass, in: ders./Pieroth (Fußn. 16), Art. 3 Rdnr. 40.

86 BVerfGE 22, 349, 361.

87 Zwischenbericht 2011 (Fußn. 4), S. 24 ff. 


\section{a) Vorzüge einer einheitlichen Regelung per se}

Die Vorzüge einer einheitlichen Regelung ergeben sich schon logisch aus den die rechtlich relevante Ungleichbehandlung begründenden und dementsprechend nachteilig wirkenden Divergenzen im Bereich des Leistungsrechts und der Zuständigkeit. ${ }^{88}$

Zum einen würde dadurch die Abhängigkeit eines Eingliederungshilfeanspruchs von unterschiedlichen Leistungsvoraussetzungen beseitigt, zum anderen könnten die Leistungen im Falle von Mehrfachbehinderungen aus einer Hand erbracht und auf Zuständigkeitsstreitigkeiten basierende Leistungsverzögerungen, insbesondere bei Schwierigkeiten der Zuordnung der Beeinträchtigung zu einer Behinderungsart ${ }^{89}$, vermieden werden. Zwar verpflichtet $\S 14$ Abs. 1 Satz 1 SGB IX ${ }^{90}$ den angegangenen Rehabilitationsträger, innerhalb von zwei Wochen nach Eingang des Antrags bei ihm festzustellen, „ob er nach dem für ihn geltenden Leistungsgesetz für die Leistung zuständig ist [...].“91 Die Regelung soll „,durch rasche Klärung von Zuständigkeiten Nachteilen des gegliederten Systems entgegen[zu]wirken."92 Allerdings wäre dies bei einer einheitlichen Regelung überhaupt nicht erforderlich.

Hinzu kommt, dass im Falle der gerichtlichen Auseinandersetzung ein einheitlicher Rechtsweg zur Verfügung stünde. 93

\section{b) Vorzüge einer einheitlichen Regelung im SGB XII}

Für die einheitliche Regelung der Eingliederungshilfe für Kinder und Jugendliche mit Behinderungen im SGB XII wird im Wesentlichen die Vermeidung eines Zuständigkeitswechsels bei einem Übertritt in die Volljährigkeit angeführt, wenn also die Phase „Kindheit und Jugend“ i. S. d. § 7 Abs. 1 Nrn. 1 und 2 SGB VIII endet. ${ }^{94}$ Für junge

88 Siehe oben II.2.b).

89 Ein Beispiel ist Autismus: Helmut Remschmidt/Christian Frese, Aktuelle Entwicklungen bei der sozialrechtlichen Zuordnung autistischer Störungen - Zu den Folgen des Urteils des OVG Nordrhein-Westfalen vom 20.2.2002, Az: 12 A 5322/00, SGb 2006, S. 410 ff.; Reinhart Lempp, Die seelische Behinderung bei Kindern und Jugendlichen als Aufgabe der Jugendhilfe, 5. Aufl. Stuttgart 2006, S. 38 ff.; Schwengers (Fußn. 73), S. 147 ff.; Franz Dillmann/Knut-Egbert Dannat, „Forever young“ - Ewig junge Abgrenzungsprobleme zwischen Leistungen für junge behinderte Menschen nach dem SGB VIII und dem SGB XII, ZfF 2009, S. 25, 26; Fegert, in: Wiesner (Fußn. 15), § 35a Rdnr. 75 ff.; BT-Drucks. 16/12860, S. 13.

90 Dazu, dass dieser gem. § 7 Satz 1 Halbsatz 1 SGB IX mangels abweichender Regelungen auch im Zusammenhang mit $\S 10$ SGB VIII Anwendung findet: Meysen, in: Münder/ders./Trenczek (Fußn. 15), § 10 Rdnr. 47 und § 35a Rdnr. 81 ff.; Wiesner, in: ders. (Fußn. 15), § 10 Rdnr. 49; Wel$t i$, in: HK-SGB IX (Fußn. 14), § 14 Rdnr. 2.

91 Ausführlich zu dem Procedere: Welti, in: HK-SGB IX (Fußn. 14), § 14 Rdnr. 34 ff.; Peter Ulrich, Die (Nicht-)Weiterleitung des Teilhabeantrages und ihre Folgen - § 14 SGB IX als gesetzesübergreifende Nahtstelle materiell- und verfassungsrechtlicher Fragen, SGb 2008, S. 452,454 ff., 456 ff.

92 BT-Drucks. 14/5074, S. 102.

93 Zum Ganzen auch: Zwischenbericht 2011 (Fußn. 4), S. 24 ff.

94 Abwägend dazu: Greß/Rixen/Wasem (Fußn. 7), VSSR 2009, S. 43, 53 ff. 
Volljährige, mithin Personen, die das 18., aber noch nicht das 27. Lebensjahr vollendet haben (§ 7 Abs. 1 Nr. 3 SGB VIII) und die nach Maßgabe des § 41 SGB VIII unter Umständen noch Eingliederungshilfe nach $\S 35$ a SGB VIII beanspruchen können, würde die Gefahr der Entstehung von Doppelstrukturen vermieden. 95

Außerdem sprenge § 35a SGB VIII „die Logik, Systematik und die Strukturmaximen des SGB VIII“, weil dieses von seinem Selbstverständnis her ein Erziehungsgesetz sei. ${ }^{96}$ Darüber hinaus werde mit $\S 35$ a SGB VIII allein für die Gruppe der seelisch behinderten jungen Menschen ein individueller Rechtsanspruch eröffnet, während im Übrigen SGB VIII - dem Gesamtkonzept des Gesetzes entsprechend - immer die Eltern beziehungsweise die Personensorgeberechtigten leistungsberechtigt seien. ${ }^{97}$

\section{c) Vorzüge einer einheitlichen Regelung im SGB VIII (,,große Lösung “)}

Für eine Zusammenfassung der Eingliederungshilfe für Kinder und Jugendliche mit Behinderungen unter dem Dach des SGB VIII spricht zum einen die gesetzessystematische Erwägung, dass die Verankerung der Eingliederungshilfe im SGB XII an sich nicht konsistent ist. Hinzu kommt die größere Sachnähe des SGB VIII.

aa) Die gesetzliche Verortung der Eingliederungshilfe im SGB XII ${ }^{98}$

Grundgesetzlicher Kompetenztitel für die Sozialhilfe ist Art. 74 Abs. 1 Nr. 7, der von „öffentlicher Fürsorge“ spricht. Darunter ist grundsätzlich die behördliche Unterstützung Hilfebedürftiger zu verstehen, die sich in einer individuellen Notlage befinden. 99 Die Sozialhilfe als „das letzte Netz der sozialen Sicherung“100 ist daher der Prototyp öffentlicher Fürsorge. Ihre Aufgabe ist es gem. § 1 Satz 1 SGB XII, „den Leistungsberechtigten die Führung eines Lebens zu ermöglichen, das der Würde des Menschen entspricht" (vgl. auch § 9 SGB I).

Besondere Aufgabe der Eingliederungshilfe ist es hingegen gem. §53 Abs. 3 Satz 1 SGB XII, „eine drohende Behinderung zu verhüten oder eine Behinderung oder deren Folgen zu beseitigen oder zu mildern ${ }^{101}$ und die behinderten Menschen in die Gesellschaft einzugliedern." Hierzu gehört nach Satz 2 insbesondere, den behinderten Men-

95 Zwischenbericht 2011 (Fußn. 4), S. 26.

96 So die Begründung der Bundesregierung zum „Entwurf eines Gesetzes zur Entlastung der Kommunen im sozialen Bereich (KEG)“, BT-Drucks. 15/4532, S. 14.

97 BT-Drucks. 15/4532, S. 14.

98 Siehe bereits: Banafsche (Fußn. 13), SGb 2012, S. 373, 376 ff.

99 Peter Lerche, Verfassungsfragen um Sozialhilfe und Jugendwohlfahrt - Ein Rechtsgutachten, Berlin 1963, S. 16, 44; ferner: Christoph Degenhart, in: Sachs (Fußn. 28), Art. 74 Rdnr. 35; Stefan Oeter, in: von Mangoldt/Klein/Starck (Fußn. 17), Art. 74 Rdnr. 55; Rupert Stettner, in: Horst Dreier (Hrsg.), Grundgesetz, Bd. II, 2. Aufl. Tübingen 2006, Art. 74 Rdnr. 44.

100 Gerhard Igl/Felix Welti, Sozialrecht, 8. Aufl. Neuwied 2007, § 55 Rdnr. 1.

101 Nähere Ausgestaltung durch die Leistungen zur medizinischen Rehabilitation gem. § 54 Abs. 1 Satz 1 SGB XII i. V. m. $\S \S 26$ bis 32 SGB IX. 
schen die Teilnahme ${ }^{102}$ am Leben in der Gemeinschaft zu ermöglichen oder zu erleichtern 103 , ihnen die Ausübung eines angemessenen Berufs oder einer sonstigen angemessenen Tätigkeit zu ermöglichen 104 oder sie so weit wie möglich unabhängig von Pflege zu machen. 105 Es geht also bei behinderungsbedingten Leistungen - nicht zuletzt angesichts der Entwicklungen der vergangenen 20 Jahre, beginnend mit der Einfügung des Art. 3 Abs. 3 Satz 2 in das Grundgesetz durch das Gesetz zur Änderung des Grundgesetzes vom 27. Oktober 1994106 - weniger um Fürsorge als vielmehr um einen Ausgleich gesellschaftlicher beziehungsweise umweltbedingter Nachteile. ${ }^{107}$ Diese Entwicklung weg vom Fürsorgedenken und hin zu einer menschenrechtlichen Betrachtungsweise von behinderungsbedingt zu gewährenden Leistungen hat in der UN-BRK mit ihren Paradigmata der Selbstbestimmung und Teilhabe (Art. 3 Buchstaben a und c), die bereits dem SGB IX zugrunde liegen (vgl. § 1) und in Wechselwirkung zueinander stehen ${ }^{108}$, abermals einen starken Ausdruck gefunden. 109

Schließlich geht auch das durch $\S 53$ Abs. 3 Satz 2 SGB XII bereits angedeutete Leistungsspektrum der Eingliederungshilfe, wie es die $\S \S 54$ bis 56 SGB XII i. V. m. $\S \S 6$ bis 24 EinglH-VO abbilden, in seiner Breite über die nach den übrigen teilhaberelevanten Leistungsgesetzen ${ }^{110} \mathrm{zu}$ gewährenden Hilfen hinaus und entfaltet insoweit eine eigenständige Bedeutung 111 , der eine Zuordnung zu der Sozialhilfe nicht gerecht zu werden vermag. ${ }^{112}$

102 Teilnahme setzt demgemäß die Möglichkeit zur Teilhabe voraus, so dass Teilhabeschranken zu Teilnahmehindernissen führen. $\mathrm{Zu}$ dem Verhältnis von Teilhabe und Teilnahme auch: Klaus Lachwitz, in: HK-SGB IX (Fußn. 14), Anhang 2 Rdnr. 5.

103 Nähere Ausgestaltung durch die Leistungen zur Teilhabe am Leben in der Gemeinschaft gem. §54 Abs. 1 Satz 1 SGB XII i. V. m. $\S \S 55$ bis 59 SGB IX.

104 Nähere Ausgestaltung durch die Leistungen zur Teilhabe am Arbeitsleben gem. §54 Abs. 1 Satz 1 SGB XII i. V. m. $\S \S 33$ bis 43 SGB IX.

105 Dies entspricht der Zielsetzung des SGB IX, die Selbstbestimmung von Menschen mit Behinderungen sowie ihre ,gleichberechtigte Teilhabe am Leben in der Gesellschaft zu fördern, Benachteiligungen zu vermeiden oder ihnen entgegenzuwirken“ ( $\$ 1$ Satz 1 ), an welcher sich die Leistungen zur Teilhabe nach Maßgabe des $₫ 4$ Abs. 1 SGB IX ausrichten.

106 BGBl. I 1994, S. 3146.

107 Antje Welke, Die Zukunft der Eingliederungshilfe für Menschen mit Behinderung, NDV 2009, S. 456, 457; Wahrendorf, in: Grubelders. (Fußn. 14), § 53 Rdnr. 35.

108 Für die UN-BRK: Heiner Bielefeldt, Zum Innovationspotenzial der UN-Behindertenrechtskonvention, Essay No. 5, 3. Aufl. Berlin 2009, S. 10 f.; stellvertretend für das SGB IX: Welti, in: HKSGB IX (Fußn. 14), § 1 Rdnr. 12, 14.

109 Dazu auch: Stefan Kurzke-Maasmeier, Von der Fürsorge zur Selbstbestimmung - Die UNBehindertenrechtskonvention als Herausforderung für Soziale Dienste, Soziale Professionen und Gemeinwesen, S. 1 ff., abrufbar unter: http://www.imew.de/fileadmin/Dokumente/Volltexte/ Tagungen_2009/UN-Konvention_25.06.09/Kurzke-Maasmeier.htm (letzter Zugriff: 18.03.2012).

110 Ausgehend von den $\S \S 6$ und 6a SGB IX sind dies das SGB V, das SGB III, das SGB VII, das SGB VI und das ALG, das BVG, das SGB VIII, das SGB XII und das SGB II.

111 Die eigenständige Bedeutung der Eingliederungshilfe nach dem SGB XII insbesondere hinsichtlich der Leistungen zur Teilhabe am Leben in der Gemeinschaft herausstellend: Felix Welti, Systematische Stellung des SGB IX im Sozialgesetzbuch - Zusammenarbeit der Leistungsträger und Koordi- 
Bei dem Einwand, § 35a SGB VIII füge sich nicht in die Systematik des SGB VIII ein ${ }^{113}$, wird neben der dargelegten systematischen Inkonsistenz von Eingliederungshilfe und Sozialhilfe in der Sache nicht hinreichend berücksichtigt, dass das SGB VIII nicht nur die Erziehung junger Menschen, sondern gleichermaßen ihre Entwicklung fokussiert. ${ }^{114}$ Dabei geht es gem. $§ 1$ Abs. 3 Nr. 1 SGB VIII um die individuelle und soziale Entwicklung, die es zu fördern und im Rahmen derer es Benachteiligungen zu vermeiden oder abzubauen gilt, was mit den Zielen der Eingliederungshilfe nach dem oben Gesagten gerade übereinstimmt.

Auch die Anspruchsberechtigung der Kinder und Jugendlichen selbst durch $\S 35 \mathrm{a}$ Abs. 1 Satz 1 SGB VIII steht dem Gesamtkonzept des SGB VIII nicht entgegen. ${ }^{115}$ Dies belegen im Besonderen die Anspruchsleistungen nach $\S 13$ Abs. 1 SGB VIII auf sozialpädagogische Hilfen ${ }^{116}$, nach $\S 18$ Abs. 3 Satz 1 SGB VIII auf Beratung und Unterstützung bei der Ausübung des Umgangsrechts nach § 1684 Abs. 1 BGB, nach $\S 24$ Abs. 1 Satz 1 SGB VIII auf Besuch einer Tageseinrichtung sowie nach $\S 41$ Abs. 1 Satz 1 SGB VIII auf Hilfe für junge Volljährige. Dass eine leistungsrechtliche Adressierung junger Menschen als Anspruchsberechtigte im Gesamtkonzept des SGB VIII im Gegenteil sogar angelegt ist, macht auch die allgemeine Vorschrift des § 8 SGB VIII deutlich, die für die Wahrnehmung aller Aufgaben der Jugendhilfe - gem. § 2 SGB VIII sind dies Leistungen und andere Aufgaben - gilt ${ }^{117}$ und in Absatz 3 Satz 1 unter den dort genannten Voraussetzungen einen Anspruch für Kinder und Jugendliche auf Beratung ohne Kenntnis der Personensorgeberechtigten i. S. d. § 7 Abs. 1 Nr. 5 SGB VIII statuiert; dass nun eine übergeordnete Norm ihrerseits Vorgaben enthalten soll, die dem Gesamtkonzept des SGB VIII zuwiderlaufen, erhellte nicht.

nierung der Leistungen, SGb 2008, S. 321, 328; Felix Welti, Die Rehabilitation im System des Sozialleistungsrechts aus rechtswissenschaftlicher Sicht, in: ders. (Hrsg.), Das Rehabilitationsrecht in der Praxis der Sozialleistungsträger, Berlin 2009, S. 16, 28.

112 Dass es sich bei der Eingliederungshilfe um eine sozialleistungsübergreifende Hilfe handelt, lässt sich auch an ihrem Verhältnis zum SGB II festmachen: Banafsche (Fußn. 13), SGb 2012, S. 373 , 377.

113 BT-Drucks. 15/4532, S. 14, siehe oben B.III.2.

114 Von einem vollständigen Übersehen kann wohl nicht ausgegangen werden, weil die Entwurfsbegründung zumindest wie folgt ausführt: „Ansatz des SGB VIII ist, die Entstehung und Lösung von Problemen des Aufwachsens, der Erziehung und der Entwicklung junger Menschen in ihrer wechselseitigen Abhängigkeit als multifaktorielle Erziehungs- und Entwicklungsbedingungen zu sehen“, BT-Drucks. 15/4532, S. 14.

115 So aber: BT-Drucks. 15/4532, S. 14, siehe oben II.3.b).

$116 \mathrm{Ob}$ es sich bei $\S 13$ Abs. 1 SGB VIII lediglich um eine objektive Gewährleistungspflicht des Trägers der öffentlichen Jugendhilfe handelt, wie es der Normwortlaut auf den ersten Blick nahelegt (,jungen Menschen [...] sollen [...] angeboten werden“), oder um ein subjektiv-öffentliches Recht, weil es um die „Überwindung individueller Beeinträchtigungen“ geht, ist umstritten. Mit weiteren Nachweisen dazu: Jutta Struck, in: Wiesner (Fußn. 15), § 13 Rdnr. 7.

117 Meysen, in: Münder/ders./Trenczek (Fußn. 15), § 8 Rdnr. 1. 


\section{bb) Größere Sachnähe des SGB VIII}

Legt man nach dem Gesagten einerseits zugrunde, dass behinderungsbedingte Leistungen nicht (mehr) als Fürsorgeleistungen, sondern als Ausgleich für gesellschaftliche beziehungsweise umweltbedingte Nachteile zu begreifen sind ${ }^{118}$, und berücksichtigt andererseits, dass Leistungen der sozialen Förderung - wie der Kinder- und Jugendhilfe nach dem SGB VIII - gerade auf die Herstellung sozialer Chancengleichheit und ,zivilisatorischer Teilhabe [...] über das Existenzminimum hinaus“ zielen, während die soziale Hilfe - wie die Sozialhilfe nach dem SGB XII - allein der Absicherung des Existenzminimums im Sinne einer der Würde des Menschen entsprechenden Lebensführung (vgl. $\S 1$ Satz 1 SGB XII und $\S 1$ Abs. 1 SGB II jeweils i. V. m. Art. 1 Abs. 1, Art. 20 Abs. $1 \mathrm{GG}^{119}$ ) dient ${ }^{120}$, kommt man im Hinblick auf Eingliederungshilfeleistungen für Kinder und Jugendliche nicht umhin, dem SGB VIII die größere Sachnähe beizumessen. ${ }^{121}$ Zum gleichen Ergebnis gelangt auch der 13. Kinder- und Jugendbericht, der die Leistungen für Kinder und Jugendliche mit Behinderungen an der Lebenslage „Kindheit und Jugend" und nicht an dem Vorliegen einer Behinderung ausgerichtet wissen will. 122

Die Jugendämter wiederum orientieren sich bei der Durchführung der Leistungen an den allein auf die Bedürfnisse der Kinder und Jugendlichen sowie Familien insgesamt zugeschnittenen Grundsätzen der Kinder- und Jugendhilfe (vgl. § 1 Abs. 3 SGB VIII als „Generalklausel und Leitnorm“123). Die Pflicht zur Einbeziehung der Jugendämter bei der Aufstellung eines Gesamtplans zur Durchführung der einzelnen Leistungen gem. $\S 58$ SGB XII ${ }^{124}$ vermag die originär jugendhilferechtliche Hilfeplanung nach $\S 36$ Abs. 2 bis 4 SGB VIII nicht zu ersetzen, der in Absatz 2 Satz 2, die Ausgestaltung der Hilfe betreffend, entscheidende Maßgaben festlegt. 125

118 Siehe oben II.3.c)aa).

$119 \mathrm{Zu}$ dem Grundrecht auf Gewährleistung eines menschenwürdigen Existenzminimums aus Art. 1 Abs. 1 i. V. m. Art 20 Abs. 1 GG: BVerfGE 40, 121, 133; 45, 187, 228; 82, 60, 85; 113, 88, 108 f.; 123, 267, 362 f.; 125, 175, 222 ff.; für die Literatur: Starck, in: von Mangoldt/Klein/ders. (Fußn. 19), Art. 1 Rdnr. 41.

120 Hans Friedrich Zacher, Entwicklung einer Dogmatik des Sozialrechts, in: Maximilian Wallerath (Hrsg.), Fiat iustitia - Recht als Aufgabe der Vernunft - Festschrift für Peter Krause zum 70. Geburtstag, Berlin 2006, S. 3, 11; in der Sache auch: Felix Welti, Das Recht auf Teilhabe behinderter Menschen in seinem verfassungs- und europarechtlichen Rahmen und im Spannungsfeld der neueren Sozialgesetzgebung, RsDE 62 (2006), S. 1, 15.

121 Vgl. bereits in der Sache: BT-DruckS. 11/5948, S. 53.

122 BT-Drucks. 16/12860, S. 12.

123 BT-Drucks. 11/5948, S. 47.

124 Vgl.: Bieritz-Harder, in: LPK-SGB XII (Fußn. 14), § 58 Rdnr. 3.

125 Der Rechtscharakter des $\S 36$ Abs. 2 Satz 2 SGB VIII wird nicht einheitlich bewertet: Von einem „subjektiv-rechtlichen Anspruch auf weitere qualifizierte Beteiligung an der Entscheidungsfindung im Hilfeplanprozess“" spricht: Meysen, in: Münder/ders./Trenczek (Fußn. 15), § 36 Rdnr. 22; dagegen für ein Zugestehen ,rein jugendamtsinterne[r] und ,verfahrensdienende[r] 'Aufgaben und Funktionen“: Reinhard Joachim Wabnitz, Rechtsansprüche gegenüber Trägern der öffentlichen Kinder- 


\section{d) Ergebnis}

Aus den genannten gesetzessystematischen Erwägungen sowie aufgrund der größeren Sachnähe des SGB VIII nicht nur zu der Lebenslage „Kindheit und Jugend“, sondern auch zu dem Ziel der Eingliederungshilfe, behinderungsbedingte Nachteile auszugleichen und auf diese Weise soziale Chancengleichheit und gesellschaftliche Teilhabe zu generieren, ist eine einheitliche Regelung der Eingliederungshilfe für Kinder und Jugendliche mit Behinderungen im SGB VIII, mithin die ,große Lösung“, zu präferieren. ${ }^{126}$

\section{Schluss}

An das unter B.III. gefundene Ergebnis anknüpfend, soll nun erneut auf den eingangs zitierten Ausschnitt aus dem 13. Kinder- und Jugendbericht rekurriert werden, der die Lebenslage „Kindheit und Jugend“ in den Vordergrund rückt und die „Behinderung“ als sekundäres Moment wertet. ${ }^{127}$

Gleiches ergibt sich bereits aus der Stellungnahme des Bundesrates zu dem „Entwurf eines Sozialgesetzbuchs - Neuntes Buch - (SGB IX) Rehabilitation und Teilhabe behinderter Menschen“, konkret zu $§ 4$ Abs. 3 SGB IX (§ 1 Abs. 2 der Entwurfsfassung), wonach „Leistungen für behinderte oder von Behinderung bedrohte Kinder [...] so geplant und gestaltet [werden], dass nach Möglichkeit Kinder nicht von ihrem sozialen Umfeld getrennt und gemeinsam mit nicht behinderten Kindern betreut werden können. Dabei werden behinderte Kinder alters- und entwicklungsentsprechend an der Planung und Ausgestaltung der einzelnen Hilfen beteiligt und ihre Sorgeberechtigten intensiv in Planung und Gestaltung der Hilfen einbezogen." In besagter Stellungnahme heißt es: „Die Standards des SGB VIII sollten als Gestaltungsauftrag auch für das SGB IX genannt werden."128

Das SGB IX schafft damit in Bezug auf Kinder und Jugendliche mit Behinderungen selbst die Anbindung an die Maßgaben des SGB VIII, ohne zwischen Behinderungsarten zu unterscheiden. In Anbetracht dessen ist die größere Sachnähe des SGB VIII zu

und Jugendhilfe nach dem Achten Buch Sozialgesetzbuch (SGB VIII), Berlin 2005, S. 210 f.; siehe auch: BT-Drucks. 11/5948, S. 74, wonach der Hilfeplan in erster Linie als „Instrument der Selbstkontrolle für das verantwortliche Jugendamt“ sowie als „Koordinierungsinstrument zwischen dem Jugendamt und dem Träger der Einrichtung, der im Einzelfall tätig wird“ dient.

126 Mit weiteren Nachweisen für die „große Lösung“: Banafsche (Fußn. 6), S. 116, 123, Fußn. 100; jüngst auch der Bericht über die Lebenssituation junger Menschen und die Leistungen der Kinderund Jugendhilfe in Deutschland - 14. Kinder- und Jugendbericht, BT-Drucks. 17/12200, S. 17, $370 f$.

127 BT-Drucks. 16/12860, S. 12, siehe unter I.

128 BT-Drucks. 14/5531, S. 6. 
eben dieser Lebenslage „Kindheit und Jugend“ ein wichtiges Argument für die „große Lösung".

Schließlich wird auch die zentrale Leitidee des Art. 6 Abs. 2 GG, wonach Pflege und Erziehung der Kinder das natürliche Recht der Eltern und die ihnen zuvörderst obliegende Pflicht sind, über deren Betätigung die staatliche Gemeinschaft wacht - das Kindeswohl als bei allen Kinder und Jugendliche betreffenden Maßnahmen vorrangig zu berücksichtigender Gesichtspunkt (vgl. Art. 3 Abs. 1 UN-Kinderrechtskonvention (UNKRK) und Art. 7 Abs. 2 UN-BRK) - durch das SGB VIII, welches Art. 6 Abs. 2 GG in $\S 1$ Abs. 2 wortlautidentisch wiederholt und damit in seinen Handlungsauftrag 129 einbezieht, maßgeblich ausgestaltet ${ }^{130}$ und wird somit die ,große Lösung“ zum verfassungsrechtlichen Gebot.

Abschließend sei daher der Rekurs auf den Philosophen David Hume gestattet, der 1779 schrieb: „Zahllose Universen mögen während einer Unendlichkeit verpfuscht und vermasselt worden sein, bevor dieses System sich durchsetzte; viel vergebliche Arbeit, viele erfolglose Versuche und eine langsame, aber beständige Verbesserung, ausgeführt während unendlicher Zeitalter in der Kunst der Weltenschaffung."131

Dies alsbald sagen zu können, bedeutete nicht zuletzt im Sinne des Gleichheitsrechts des Art. 3 GG, dass die Debatte um die „große Lösung“ nicht zu einer „,unendlichen Geschichte“" geworden wäre ${ }^{132}$, sondern zu einem positiven Ende gefunden hätte.

129 Vgl.: Münder, in: ders./Meysen/Trenczek (Fußn. 15), § 1 Rdnr. 1 f.

130 Zur Notwendigkeit der gesetzlichen Ausgestaltung des Elternrechts des Art. 6 Abs. 2 GG: BVerfGE 84, 168, 180; ferner: Hans Friedrich Zacher, Elternrecht, in: Josef Isensee/Paul Kirchhof (Hrsg.), Handbuch des Staatsrechts der Bundesrepublik Deutschland, Bd. VI, Heidelberg 1989, § 134 Rdnr. 48; Jarass, in ders./Pieroth (Fußn. 16), Art. 6 Rdnr. 51.

131 Zitiert nach: Marcus Chown, Das Universum nebenan - Revolutionäre Ideen in der Astrophysik, Köln 2003, S. 161.

132 Siehe den Beitrag von Wiesner, S. 153 in diesem Band. 


\title{
Kinder und Jugendliche mit Behinderungen in der Kommune - Kommentar aus Sicht eines Jugendamtes
}

\author{
Maria Kurz-Adam
}

I. Die „Große Lösung“ als Weg zur Inklusion für Kinder und Jugendliche in den Kommunen - Anmerkungen zum aktuellen Diskussionsstand in der

Kinder- und Jugendhilfe

II. Inklusion in der Kinder- und Jugendhilfe - Widersprüche und Herausforderungen

III. Fazit: Perspektiven einer inklusiven Kinder- und Jugendhilfe - neue Hilfearchitekturen für eine inklusive „Hilfe zur Entwicklung““

I. Die „,Große Lösung“ als Weg zur Inklusion für Kinder und Jugendliche in den Kommunen - Anmerkungen zum aktuellen Diskussionsstand in der Kinder- und Jugendhilfe

Die Debatten und die Literatur zur „Großen Lösung“ - also der Zusammenführung der Zuständigkeit für die Eingliederungshilfe für geistig und körperlich behinderte Kinder und Jugendliche und für seelisch behinderte Kinder und Jugendliche im Leistungssystem der Kinder- und Jugendhilfe - sind mittlerweile fast Legion. Die wesentlichen vornehmlich fachlichen - Argumente für eine solche Zusammenführung liegen insbesondere seit dem Erscheinen des 11. Kinder- und Jugendhilfeberichts auf dem Tisch: eine Reduzierung der Schnittstellen zwischen Sozialrecht und Kinder- und Jugendhilferecht, eine damit verbundene „Verminderung der Abgrenzungs-, Zuständigkeits- und Konkurrenzprobleme“" insbesondere bei Mehrfachbehinderungen im Indikationsfeld zwischen geistiger und seelischer Behinderung sind die wesentlichen fachlichen Argumente des Berichts, der die getrennten Zuständigkeiten seit Inkrafttreten des § 35a SGB VIII letztlich für gescheitert hält. Das Zurechtfinden in den unterschiedlichen Zuständigkeiten der dualen Eingliederungshilfe für Kinder- und Jugendliche ist gerade aus Betroffenensicht, und besonders aus Sicht der hilfesuchenden Eltern, zu einer Aufgabe geworden, die ohne professionelle Beratung und Unterstützung kaum mehr zu schaffen ist: im Dickicht der Rechtsbestimmungen und Vorschriften scheint der Hilfeanspruch 
selbst so zergliedert zu werden, dass er gleichsam zu verschwinden droht. Die „Große Lösung“ steht daher für ein Hilfekonzept „aus einer Hand“, das rechtliche und fachliche Gleichbehandlung verspricht und die Zugänge für die Hilfe wenn nicht erleichtert, doch zumindest klarer gestaltet.

Zugleich steht die „Große Lösung“ nahezu als Synonym für die Verwirklichung des Inklusionsgebotes der UN-Behindertenkonvention: die Zusammenführung der Zuständigkeiten in eine - möglichst kommunale - Zuständigkeit wird als Voraussetzung gesehen, um Teilhabe am gesellschaftlichen Geschehen für Kinder- und Jugendliche in einer Kommune nachhaltig zu verwirklichen. Die Kinder- und Jugendhilfe wird in dieser Perspektive als das nicht allein rechtlich, sondern zuallererst fachlich angemessene Leistungssystem für den Weg zur Inklusion beschrieben, weil es nicht die Defizite oder die Problemlage von Kindern und Jugendlichen in den Mittelpunkt stellt, sondern die Lebenslage Kindheit und Jugend. Die Unterscheidung Behinderung oder Erziehungsschwierigkeit würde - so die Argumentation - für eine inklusive Kinder- und Jugendhilfe nicht mehr erheblich sein: ausschlaggebend für die Leistungen der Kinder- und Jugendhilfe sind dann lebenslagenspezifische Sachverhalte, die die Schärfe der Diagnosestreitigkeiten verlassen würden zugunsten einer Inklusionsperspektive der Hilfen, die letztlich auch zu Entlastungen des von Überspezialisierung gekennzeichneten Hilfesystems führen würden.

Seitens der Kommunen ist in der Debatte zur „Großen Lösung“ viel dazu eingewendet worden: neben dem Argument, dass es weiterhin massive Schnittstellen zwischen dem Gesundheitssystem und der Behindertenhilfe - wo auch immer sie angesiedelt wäre - geben würde, war ein ausschlaggebendes Argument für die kommunale Seite der Verweis auf die teils dramatisch steigenden Kosten in der Erziehungshilfen der Kinderund Jugendhilfe. Selbst bei entsprechendem Finanztransfer hat die kommunale Seite Zweifel an einer effektiven Steuerungsfähigkeit der Kinder- und Jugendhilfe: seit Inkrafttreten des SGB VIII sind die Kosten in der Kinder- und Jugendhilfe, insbesondere im Bereich der Einzelfallhilfe, um ein Vielfaches gestiegen. In jüngster Zeit haben diese Zweifel einen neuen Höhepunkt erfahren, indem der Rechtsanspruch in der Kinder- und Jugendhilfe auf individuelle Hilfe - ein wesentlicher Ankerungspunkt für die Befürworter der „Großen Lösung“ - in Frage gestellt wird: in der Initiative der Behörde für Arbeit, Soziales, Familie und Integration der Freien und Hansestadt Hamburg wird unter dem Titel der „Wiedergewinnung kommunaler Handlungsfähigkeit zur Ausgestaltung von Jugendhilfeleistungen" ein Konzept beschrieben, das die Einzelfallhilfen zugunsten sozialräumlicher struktureller Leistungskonzepte zunehmend vermeiden soll. An die Stelle des individuellen Rechtsanspruchs auf individuelle Hilfe soll eine Gewährleistungspflicht der Kommunen für sozialräumliche und niedrigschwellige Unterstützungssysteme treten. Die „Große Lösung“ in einer so gedachten Kinder- und Jugendhilfe wäre dann in Teilen keine Lösung mehr für den Einzelfall und seinen individuellen Bedarf von Unterstützung, den die Kinder- und Jugendhilfe zu erbringen hätte, sondern mehr 
noch ein strukturelles Konzept für den jeweiligen Sozialraum, der so gestaltet werden muss, dass er inklusive Kraft entfalten kann.

Diese so umstrittene wie spannende Debatte zum Spannungsfeld zwischen subjektivem Rechtsanspruch und Sozialraum verweist in ihrem Kern auf Fragen der Kinderund Jugendhilfe selbst, die erheblich zu einer Differenzierung der Diskussion über die Leitungsstärke der Kinder- und Jugendhilfe und ihr Profil führen und geführt haben. Denn die Kinder- und Jugendhilfe ist - und das ist ihr großer Vorsprung gegenüber anderen Sozialsystemen - ein lebenslagenorientiertes und damit strukturell offenes System, das sich schnell auf neue Aufgaben und offene Prozesse einstellen kann. Sie ist aber - gerade im Hinblick auf den individuellen Rechtsanspruch - eben auch ein Sozialleistungssystem mit Rechtsansprüchen, unabhängigen Standards und Ansprüchen und Zielen sowie Zugangswegen, Prüfkriterien und Steuerungsparametern, die auch innerhalb der Zuständigkeit der Kinder- und Jugendhilfe eine eigene Architektur bilden. Die „unvoreingenommene, am Kindeswohl orientierte Prüfung der Leistungsvoraussetzungen im Kontext der Hilfen zur Erziehung"1 ist durch die „Große Lösung“ nicht beendet. Und schließlich wird auch das Rechts- und Hilfesystem der Kinder- und Jugendhilfe getragen von einer sozialstaatlichen Utopie, die die Vielfalt von Lebenslagen nicht in allen Belangen berücksichtigt. Das umfassende Ziel der Herstellung einer gemeinschaftsfähigen Persönlichkeit etwa bedarf im Blick auf die besondere Lebenslage schwerstbehinderter Kinder und Jugendlicher auf dem Weg zur Inklusion einer differenzierten Sicht, weil es von den behinderten jungen Menschen etwas fordert, statt sich auf deren Bedürfnisse einzustellen und damit schon Ansätze der Exklusion in sich trägt. $^{2}$

Damit wird deutlich, dass auch die Kinder- und Jugendhilfe in einer Zusammenführung der Zuständigkeiten der Eingliederungshilfe nicht bloße Rechtsvorschriften additiv ändern muss, sondern sehr wesentlich in ihrer gesamten Gestalt, ihrer „Architektur“ neu gedacht werden muss: die „Umstellung des Jugendhilfesystems auf ein System inklusiver Hilfeleistung und Unterstützung für behinderte wie nicht behinderte junge Menschen“ bedeute - so die klare Expertenmeinung - einen „tief greifenden Einschnitt in die bisherigen Strukturen der Kinder- und Jugendhilfe ${ }^{\text {(633. }}$. Die Kinder- und Jugendhilfe kann sich also nicht auf der Debatte zur „Großen Lösung“ mit dem Verweis auf außerhalb ihres Zuständigkeitsbereichs liegende Probleme ausruhen. Jenseits aller rechtlichen Debatten bleibt die „Große Lösung“ ein Synonym für Inklusion, die auch das System der Kinder- und Jugendhilfe in den Kommunen vor eine enorme Herausforderung stellt und einen Paradigmenwechsel fordert, der tief in die Strukturen der Angebote und Hilfen reicht.

1 Mitteilungsblatt Bayerisches Landesjugendamt 3-4, 2012, S. 2.

2 Vgl. Gerlach/Hinrichs, Inklusion und die „große Lösung“ für die Jugend- und Behindertenhilfe (Teil 2), ZKJ 2012, S. 134.

3 Vgl. Gerlach/Hinrichs, Inklusion und die ,große Lösung“ für die Jugend- und Behindertenhilfe (Teil 1), ZKJ 2012, S. 86. 


\section{Inklusion in der Kinder- und Jugendhilfe- Widersprüche und Herausforderungen}

Die Debatte um Inklusion in der Kinder- und Jugendhilfe und die Umsetzung der UN-Konvention in diesem tiefgreifenden Verständnis eines anstehenden Paradigmenwechsels ist an vielen Orten erst zu beginnen. Die Frage „Wie inklusiv ist die Kinderund Jugendhilfe" ist derzeit eher zufällig und nur für einzelne Arbeitsfelder in der Kinder- und Jugendhilfe und von Kommune zu Kommune unterschiedlich beantwortet; gemeinsam ist dieser Antwort vor allem, dass sie gerade im Blick auf die Einzelfallhilfen und die Hilfen zur Erziehung und ihre Verbindung mit den Leistungen der Kinderund Jugendhilfe als Eingliederungshilfe für seelisch behinderte junge Menschen auf Widersprüche stößt. Hier stehen vielfach die Diskussionen zu einer Verwirklichung von Inklusion noch am Anfang und haben die Fachlichkeit und die Angebotslandschaft mit dem Verweis auf die rechtliche Lage, die Begrenztheit der Zuständigkeiten und den individuellen Rechtsanspruch noch wenig berührt.

Deutlich intensiver ist die Inklusion in den Arbeitsfeldern der offenen Kinder- und Jugendhilfe fortgeschritten. Hier finden sich seit Jahren Inklusionsansätze in vielen kleineren und größeren Projekten und insbesondere bei den Ferien- und Freizeitangeboten: die offene Kinder- und Jugendarbeit etwa in allen ihren Facetten hat in München in guter Weise Angebote entwickelt, in die der Inklusionsgedanke eingeschrieben ist. Grundlage dieser Projekte sind die Leitlinien der Landeshauptstadt München für die Arbeit mit Kindern und Jugendlichen mit und ohne Behinderung, die das Stadtjugendamt mit vielen beteiligten stadtweiten Dienststellen entwickelt hat und inklusiv auch in einfacher Sprache und auf CD erhältlich sind ${ }^{4}$. Umsetzungsprojekte finden sich etwa in inklusiven Einrichtungen oder in Projekten, die das Bewusstsein in der Bevölkerung und der Einrichtungen für alltägliche Barrieren schärfen ${ }^{5}$. Umsetzungsformen finden sich aber auch in geschlechtsspezifischen Projekten: etwa in der Mädchenarbeit und Mädchenbildung, die gerade in der Berufsorientierung von Mädchen inklusive Beratung und Schulung anbieten oder auch Angebote zur Prävention von sexuellem Missbrauch an Kindern mit und ohne Behinderung. ${ }^{6}$

Deutlich ist schon jetzt, dass eine inklusive offene Arbeit trotz aller Anstrengungen einer fachlichen und strukturellen Umorientierung nicht umsonst zu haben ist: insbesondere die Anforderungen für mehr Koordination zwischen den Angeboten, mehr individuelle Begleitung von behinderten Kindern und Jugendlichen, die notwendige laufen-

4 Landeshauptstadt München, Sozialreferat - Stadtjugendamt: Leitlinien für die Arbeit mit Kindern und Jugendlichen mit und ohne Behinderung, München 2007.

5 Hier sei beispielhaft verwiesen auf das Projekt des Kreisjugendrings München: „Auf Herz und Rampen prüfen“" oder das Modellprojekt MOP integrativer Jugendtreff.

6 Hier sei verwiesen auf die vom Stadtjugendamt München geförderten Projekte mira Mädchenbildung Schule und Beruf e.V. und AMYNA zur Prävention von sexuellem Missbrauch. 
de Fortbildungsarbeit für die sozialpädagogische Fachlichkeit und einer inklusiven Öffentlichkeitsarbeit - wie etwa die Darstellung in einfacher Sprache - stellen die Kommunen vor neue Aufgaben.

Doch gerade im Hinblick auf die individuellen Rechtsansprüche der Eingliederungshilfe in der Kinder- und Jugendhilfe für seelisch behinderte Kinder werden auch Widersprüche sichtbar, die seit langem die Kommunen beschäftigen. Denn die Eingliederungshilfe hat sich in allen ihren Formen, insbesondere aber im teilstationären und stationären Bereich, mittlerweile zu einem derart hoch spezialisierten System entwickelt, das es in sich selbst schon erhebliche Schnittstellen aufweist und Barrieren bildet. Das Spannungsfeld zwischen individuell zugeschnittener und schützender Hilfe und einem sozialen Inklusionsanspruch tritt in diesem Handlungsfeld der Kinder- und Jugendhilfe scharf hervor. Eingliederungshilfe im Kontext der Erziehungshilfe wird in der Kinderund Jugendhilfe vielfach als Schutz- und Schonraum vor den Anforderungen des Bildungssystems und den Gefahren sozialer Ausgrenzung gerade der psychosozial auffälligen jungen Menschen gedacht. Sie findet vielfach nicht inklusiv im System der Schule statt, sondern allenfalls am System und vielfach außerhalb als schulentlastendes und förderndes Angebot. Die hoch spezialisierten Angebote in den Hilfen zur Erziehung im Rahmen des $\S 35$ a SGB VIII sind in den vergangenen Jahren um ein Vielfaches gestiegen: statt einer Inklusion hat zunehmende Ausdifferenzierung das Feld bestimmt.

Getrennte Lern- und Förderorte sind neben vielen anderen Ursachen auch ein Produkt dieses Schutz- und Hilfegedankens mit allen seinen Vorteilen, aber auch Nachteilen, die zunehmend auch von den Eltern gesehen werden. Viele Eltern behinderter Kinder wollen diese Trennung nicht mehr; sie greifen die Inklusionsdebatte offensiv auf und wünschen sich zunehmend einen Weg für ihre Kinder, der nicht von Anfang an alle Zeichen eines Sonderweges in sich trägt. Seit der Änderung des Art. 30a Abs. 8 des Bayerischen Erziehungs- und Unterrichtsgesetzes ist die Forderung nach einem gemeinsamen Lern- und Förderort auch seitens der Eltern lauter geworden. Der Lernort Schule steht hier vor gewaltigen Herausforderungen, dem Wunsch der Eltern nach einer inklusiven Bildung ihrer Kinder nachzukommen und die Kinder in einer „Schule für alle“ zu halten, statt die Lern- und Förderorte zu trennen. Diese Herausforderung trifft aber nicht allein die Schule, sondern ebenso die örtlichen Jugendämter. Deutlichster Indikator dafür ist die sprunghaft wachsende Zahl von Anträgen und Maßnahmen auf Schulbegleitung, die mittlerweile ein deutlich erweitertes Indikationsfeld von Behinderungsarten umfasst. Die Kosten haben sich - so jüngste Schätzungen bayernweit - verdreifacht und stellen gerade kleine Jugendämter vor große Herausforderungen ${ }^{7}$. Zugleich wird deutlich, dass das Konzept „Schulbegleitung“ als individuelles Leistungsangebot an seine Grenzen kommt, wenn nicht auch seitens der Kinder- und Jugendhilfe neue umfassendere Konzepte einer inklusiven Umgestaltung ihrer Angebote im gesamten Leistungsfeld für seelisch behinderte Kinder entwickelt werden. Nicht allein besteht die Gefahr,

7 Vgl. Mitteilungsblatt Bayerisches Landesjugendamt 3-4, 2012, S. 1. 
dass sich die Kinder- und Jugendhilfe zunehmend mehr in spezialisierte diagnostische Fragen begibt, mehr noch wird deutlich, dass eine additive Reihung von Leistungstatbeständen, wie sie derzeit im SGB VIII vorgesehen sind, in der Kinder- und Jugendhilfe das Ziel einer wirklichen Umgestaltung ihrer Architektur verfehlen würde und eine wirkliche Veränderung einer inklusiven Kultur des Aufwachsens und der inklusiven Bildung nicht erreicht würde. Statt getrennter Lern- und Förderorte sind gerade in den Einzelfallhilfen neue Wege erforderlich, die sowohl eine neue, selbstverständlich werdende Zusammenarbeit mit den Schulen als auch eine neue Zusammenarbeit mit den Unterstützungsnetzen der Familien und nicht zuletzt auch eine Veränderung der Infrastruktur im Sozialraum erfordern. Wenn - wie dies Reinhard Wiesner 8 in seinem Tagungsbeitrag formuliert hat - durch die „Große Lösung“ ein neuer Leistungstatbestand entstehen wird im Schnittfeld zwischen Hilfe zur Entwicklung, Hilfe zur Erziehung und Teilhabe, so wird dies auch die Angebotsstruktur der Hilfen zur Erziehung nachhaltig verändern. Vom „Kind aus gedacht“ müssen Spezialisierungen überdacht, Kooperationen deutlich näher konzipiert und ineinander verwoben werden, Ressourcen auch neu verteilt werden. Die klassischen Eingliederungshilfen gerade im Kontext der Hilfen zur Erziehung in der Kinder- und Jugendhilfe stehen hier vor neuen und spannenden Herausforderungen, dieses offene Feld an Hilfe- und Teilhabemöglichkeiten auszugestalten.

\section{Fazit: Perspektiven einer inklusiven Kinder- und Jugendhilfe - neue Hilfearchitekturen für eine inklusive ,Hilfe zur Entwicklung“}

Inklusion in der Kinder- und Jugendhilfe ist damit zu einer Kernfrage geworden, die sowohl das System der Kinder- und Jugendhilfe, aber mehr noch seine Bezüge zur Schule und zum Gesundheitssystem herausfordert. Die kommunale Kinder- und Jugendhilfe muss, wenn sie diese Kernfrage bewältigen will, unbestritten hier verdeutlichen, dass sie ohne eine inklusive Veränderung aller staatlichen Leistungssysteme überfordert ist: die Inklusionsdebatte ist eine gesamtgesellschaftliche Aufgabe, mit der die Jugendämter auch bei einer veränderten Rechtslage der „Großen Lösung“ nicht allein gelassen werden dürfen. Unbestritten ist aber auch, dass die Kinder- und Jugendhilfe in ihrem eigenen System vor einem erheblichen Umbau ihrer Angebote steht, wenn sie den Weg der Inklusion fördern und weiterentwickeln will. Die Modelle, die in der offenen Kinder- und Jugendarbeit entwickelt worden sind, zeigen diese Anforderungen deutlich auf. Notwendig sind eine inklusive Haltung der Fachkräfte, eine neue Zusammenarbeit mit den Eltern und vor allem eine ausgeprägte Beteiligung der Kinder und Jugendlichen. Gerade im Bereich der Hilfen zur Erziehung sind neue individuelle und infrastrukturelle Hilfearchitekturen notwendig, um Hilfen in der Lebenswelt zu ermöglichen

8 Siehe S. 153 in diesem Band. 
und strukturelle Trennungen aufzuheben oder zu lösen. Die wohl größte Herausforderung besteht für die Zukunft in einem neuen Zusammenwirken von Jugendhilfe und Schule, das vom Subjekt aus gedacht wird, um Inklusion zu verwirklichen. Dies kann auch bei einer „Großen Lösung“ nicht in einem immer neuen Ausbau oder Hinzufügen von spezialisierten Angeboten geschehen, sondern zu aller erst in einer lebendigen und kritischen Sichtung dessen, was die Kinder- und Jugendhilfe heute anbietet. Im Blick auf die heute schon bestehenden Probleme zunehmender Spezialisierung und einer damit verbundenen Gefahr der Ausgrenzung kann die „Große Lösung“ auch als Lernprozess für die Kinder- und Jugendhilfe selbst verstanden werden. Insofern sind die Gedanken der Hamburger Initiative nicht vorschnell zu verwerfen: sie sind vielmehr Anstoß, über systembedingte Ausgrenzungs- und Stigmatisierungseffekte nachzudenken, die ein am individuellen Rechtsanspruch ausgerichtetes Angebot möglicherweise miterzeugt. Auch in einem Leistungsbereich, der dem individuellen Rechtsanspruch auf Eingliederungshilfe und Hilfe zur Erziehung und - später möglicherweise - Hilfe zur Entwicklung unterliegt, ist Weiterentwicklung notwendig, wenn Inklusion in die $\mathrm{Zu}$ kunft gedacht werden soll. Möglichkeiten, die individuellen Hilfen in der Kinder- und Jugendhilfe achtsam auf den Weg der Inklusion zu bringen, bestehen schon jetzt. Ideen dazu sind schon jetzt vielfach da: etwa die präventive Nutzung von Kindergartenplätzen als Hilfe eines Hilfeplans mit besonderen individuellen Unterstützungsformen für psychosozial hochbelastete Kinder, der Ausbau der Sozialpädagogischen Lernhilfen mit einem besonderen Profil, um seelisch behinderten Kindern niedrigschwellige Unterstützung im Alltag zu ermöglichen, Konzepte einer flexiblen heilpädagogischen Eingliederungshilfe im Schnittfeld zwischen Schule und Heilpädagogischer Tagesstätte oder Modelle eines gemeinsamen Lern- und Förderortes von Schule und Jugendhilfe wie sie schon jetzt in einigen Kommunen im Zusammenwirken von Schule und Hort im Rahmen des Ganztages verwirklicht werden. Neue Hilfearchitekturen können schon jetzt gedacht, schon jetzt entwickelt werden, wenn Inklusion Teil des Selbstverständnisses der Kinder und Jugendhilfe in den Kommunen ist. 


\title{
Kinder und Jugendliche mit Behinderungen in der Kommune - Kommentar aus Sicht einer Schule
}

\author{
Martina Weide-Gertke und Brigitta Seidenschwang
}

Das Ziel einer inklusiven Schule, dem sich auch die Ernst-Barlach-Schulen der Stiftung Pfennigparade verpflichtet haben, muss es sein, den Schülerinnen und Schülern größtmögliche Selbstständigkeit für ihre körperliche, geistige und soziale Entwicklung zu eröffnen. Es geht darum, sie auf eine möglichst realistische Einschätzung ihrer eigenen Möglichkeiten und Fähigkeiten, eine qualifizierte Berufswahl, den Übertritt in weitere schulische Bildungsgänge bis zur Hochschulreife, eine Teilhabe am gesellschaftlichen Leben und eine eigenständige Lebensführung vorzubereiten.

Hinzukommen muss außerdem die Integration Nichtbehinderter, um behinderten Schülern ein möglichst alltagsnahes Umfeld zu schaffen bzw. ihnen den Umgang mit Nichtbehinderten zu ermöglichen.

Nichtbehinderte Jugendliche bringen andere Vorerfahrungen mit, die im schulischen Zusammenleben für behinderte Jugendliche entwicklungsfördernd sind. Besonders im Bereich der Selbstwahrnehmung/Einschätzung ist das Feedback durch Gleichaltrige sehr wichtig. Zudem ist die Hilfe durch Mitschüler in der Regel sozial fördernder als die Hilfe durch professionelle Erwachsene. Zu lernen ist vor allem, angemessen Hilfen einzufordern, das Nehmen und Geben und sich um weitestgehende Selbstständigkeit zu bemühen. Behinderte und nichtbehinderte Jugendliche können über das schulische $\mathrm{Zu}$ sammenleben hinaus dauerhafte Beziehungen aufbauen.

Wir erleben, dass die Begegnung zwischen behinderten und nichtbehinderten Menschen ein großes Wachstumspotential für die Entwicklung ihrer Persönlichkeit enthält.

Die Grundlagen unseres pädagogischen Handelns sind die Wahrnehmung jedes Einzelnen und der Respekt vor der Besonderheit der uns anvertrauten jungen Menschen. Wir unterstützen und fördern die Stärken unserer Schüler, denn Lernen und Entwicklung brauchen Ermutigung.

Wir schaffen einerseits Lernbedingungen, welche die Eigenaktivität und Selbstbestimmung stärken. Dazu bringen wir junge Menschen in herausfordernde Situationen, die sie auch bestehen können. Andererseits setzen wir uns mit Abhängigkeiten und Begrenztheiten auseinander.

Für den Unterricht bedeutet dies ganz konkret, flexibel mit inhaltlichen und zeitlichen Konzepten umzugehen, „Störungen" mit Geduld zu begegnen, kleinste Entwicklungsfortschritte genau zu beobachten und zu verstärken, die Schüler zu eigenständigem, selbstverantwortlichem Lernen und Arbeiten anzuleiten und Teamfähigkeit, auch in klassen- und jahrgangsstufenübergreifenden Projekten, zu fördern. 
Die Schüler müssen im Gegenzug die grundlegende Bereitschaft aufbringen, sich auf neue Inhalte interessiert einzulassen, zunehmend den Unterricht mitzugestalten, sich selbst aktiv in das Schulleben einzubringen und aufgeschlossen und unvoreingenommen auf ihre Mitschüler zuzugehen.

Auf dieser Basis werden die Kinder und Jugendlichen dabei unterstützt, ihre Stärken zu entwickeln, aber auch ihre Schwächen selbstkritisch wahrzunehmen und zu verringern.

Wichtig sind dabei auch ein respektvoller Umgang miteinander sowie die Einhaltung gepflegter Umgangsformen. Im täglichen Miteinander müssen persönliche Grenzen aufgezeigt und muss für deren Einhaltung gesorgt werden. Kritik muss fair und sachgerecht als wichtige Rückmeldung zu Inhalten oder Verhalten geäußert werden und darf keinesfalls zur Abwertung der Person führen. Die Erwartung geht dahin, dass jeder sein Handeln reflektiert und bereit ist, auch von anderen zu lernen.

Dazu ist es zwingend notwendig, Personen in ihrer Gesamtheit wertschätzend wahrzunehmen und zu achten.

Dieses bedeutet im Einzelnen, Individualität zu unterstützen, indem Talente erkannt und eigene Ideen gefördert sowie Erfahrungen miteinander reflektiert werden. Zudem müssen den Schülern Vorbilder angeboten werden, die eigene Werte leben, als Menschen sichtbar werden und als Gesprächspartner gerade auch in schwierigen Situationen helfen. Dies funktioniert nur unter Herstellung guter Rahmenbedingungen, indem grundsätzlich eine positive Einstellung zueinander gepflegt und problemlösungsorientiert gehandelt wird.

Ein lebendiges Miteinander zwischen Schülern, Lehrern, Assistenten, Eltern, Außenwohngruppen und Therapeuten gelingt, indem Spontaneität und Kreativität zugelassen und gelebt werden.

Grundlage dafür ist wiederum eine hohe Qualität der Arbeit, insbesondere Fachwissen und regelmäßige Fortbildungen in allen Bereichen pädagogischen Handelns. Wichtig sind auch der ständige Kontakt in Bayern zum Staatsministerium für Unterricht und Kultus und zu den Bezirksregierungen, der Kontakt zu anderen Schulen, insbesondere zur Grund-/Haupt-und Fachoberschule im eigenen Haus, die Umsetzung des Lehrplans für die sechsstufige Realschule in Bayern, eine kontinuierliche Schulentwicklung sowie gegenseitige kollegiale Unterstützung bei der Umsetzung aller Ziele und Teamfähigkeit.

Es muss ferner eine Zusammenarbeit mit den Schülern, Assistenten-Teams, Schulbegleitern, Erziehungsberechtigten, den Betreuern in Stationären Wohngruppen und Heilpädagogischen Tagesstätten, Ärztlichem Dienst, Physio-und Ergotherapie, Logopädie, Psychologischem Dienst, Sozial-und Beratungsdienst und schließlich externen Beratungsdiensten, wie Mobilen Sozialen Diensten, Staatlicher Schulberatung, der Bundesagentur für Arbeit und den Jugendämtern stattfinden.

Gute Arbeit gelingt nur durch eine konsequente Überprüfung und Weiterentwicklung vorhandener Kommunikationsstrukturen und Arbeitsabläufe. 
Bauliche, technische und organisatorische Voraussetzungen dafür sind barrierefreie Schulgebäude, behindertengerechte Schwimmbäder und Sporthallen, Räumlichkeiten zur Differenzierung, Schulküchen mit unterfahrbarer und höhenverstellbarer Einrichtung, verstellbare Tische und Spezialstühle, die Verwendung von technischen Hilfsmitteln, wie etwa Notebooks, Beamer, Overheadprojektoren, Lesegeräte, Kommunikatoren, im Unterricht und bei Prüfungen, ein Mittagsrestaurant, Stadtteilbibliotheken in den Schulgebäuden sowie Fahrdienste für behinderte Schüler.

Pädagogische Rahmenbedingungen dafür sind geringe Klassenstärken (ca. 15 Schüler), die Integration auch nichtbehinderter Schüler, ein Unterricht im Fachlehrersystem mit einer Unterrichtsbegleitung durch ein eigenes Assistenten-Team, welches bei Bedarf Schüler unterstützt, Nachteilsausgleiche, individuelle behinderungsbedingte Sonderregelungen, z.B. verkürzte Unterrichtszeit, Heimunterricht, Therapie statt Unterricht, die Stärkung der eigenständigen Arbeitsweise der Schüler durch unterstützende Unterrichtsformen und nicht zuletzt eine starke Binnendifferenzierung im Unterricht. 


\section{Autorenverzeichnis}

Dr. Minou Banafsche, Wissenschaftliche Mitarbeiterin am Max-Planck-Institut für Sozialrecht und Sozialpolitik, München

Prof. Dr. Ulrich Becker, LL.M. (EHI), Direktor am Max-Planck-Institut für Sozialrecht und Sozialpolitik, München

Prof. Dr. Clemens Dannenbeck, Prodekan der Hochschule Landshut

Prof. Dr. Markus Dederich, Lehrstuhl für Allgemeine Heilpädagogik, Theorie der Heilpädagogik und Rehabilitation am Department Heilpädagogik der Universität zu Köln

Prof. Dr. Gerhard Igl, Leiter des Instituts für Sozialrecht und Gesundheitsrecht und Lehrstuhl für Öffentliches Recht und Sozialrecht an der Juristischen Fakultät der Christian-Albrechts-Universität zu Kiel

Helmut Kneppe, Dezernent für Jugend, Familie und Soziales, Kreis Siegen-Wittgenstein

Dr. Andreas Kuhn, Wissenschaftlicher Referent im Deutschen Verein für öffentliche und private Fürsorge e. V., Berlin

Dr. Maria Kurz-Adam, Leiterin des Stadtjugendamtes München

Stephan Rittweger, Vorsitzender Richter am Bayerischen Landessozialgericht, München

Dr. Markus Schäfers, Referent für Teilhabeorientierte Dienstleistungen und Steuerungsinstrumente in der Eingliederungshilfe bei der Bundesvereinigung Lebenshilfe e. V., Berlin

Brigitta Seidenschwang, Fachlehrerin und Physiotherapeutin an der Real- und Fachoberschule der Ernst-Barlach-Schulen der Stiftung Pfennigparade, München 
Prof. Dr. Elisabeth Wacker, Lehrstuhl für Diversitätssoziologie an der Fakultät für Sport- und Gesundheitswissenschaft der Technischen Universität München und Max Planck Fellow am Max-Planck-Institut für Sozialrecht und Sozialpolitik, München

Prof. Dr. Gudrun Wansing, Leitung des Fachgebiets Behinderung und Inklusion am Institut für Sozialwesen der Universität Kassel

Martina Weide-Gertke, Schulleiterin der Real- und Fachoberschule der Ernst-BarlachSchulen der Stiftung Pfennigparade, München

Prof. Dr. Felix Welti, Leitung des Fachgebiets Sozialrecht der Rehabilitation und Recht der behinderten Menschen am Institut für Sozialwesen der Universität Kassel

Prof. Dr. Dr. h.c. Reinhard Wiesner, Ministerialrat a. D., Honorarprofessor an der Freien Universität Berlin 